\title{
Bifurcações de Campos Vetoriais Descontínuos
}

Anderson Luiz Maciel

TESE APRESENTADA

$\mathrm{AO}$

Instituto DE Matemática E EstatísticA

DA

Universidade de SÃo Paulo

PARA

OBTENÇÃO DO TÍTULO

$\mathrm{DE}$

DOUTOR EM

CIÊNCIAS

\author{
Programa: Matemática Aplicada \\ Orientador: Prof. Dr. Jorge Manuel Sotomayor Tello
}

Durante o desenvolvimento deste trabalho o autor recebeu auxílio financeiro da CAPES

São Paulo, agosto de 2009 


\section{Bifurcações de Campos Vetoriais Descontínuos}

Este exemplar corresponde à redação final da tese devidamente corrigida e defendida por (Anderson Luiz Maciel) e aprovada pela Comissão Julgadora.

Banca Examinadora:

- Prof. Dr. Clodoaldo Grotta Ragazzo (Presidente) - IME-USP.

- Prof. Dr. Orlando Francisco Lopes - IME-USP.

- Prof. Dr. Cláudio Gomes Pessoa - UFU.

- Prof. Dr. Luis Fernando de Osório Mello - UNIFEI.

- Prof. Dr. Ronaldo Garcia - UFG. 


\section{Agradecimentos}

Inicialmente gostaria de agradecer ao Prof. Sotomayor pela orientação. Foi uma honra para mim ser aluno do Prof. Soto com quem aprendi muito ao longo desses anos, e devo dizer que a minha admiração a ele crescia a cada vez que tomava conhecimento dos seus trabalhos e a cada reunião que fazíamos para discutir o trabalho que originou essa tese.

À minha família pelo suporte e pela paciência, principalmente a minha esposa Divane, meus pais Vicente e Nádia, meus irmãos Cris e Ale, minha cunhada Veridiana, ao Gabriel à Isabela e a todos os meus parentes.

Agradeço aos colegas do IME principalmente pelas conversas, relacionadas ou não a matemática, na sala do café ou nos corredores do IME que tornaram esse período de doutorado mais suportável.

Agradeço aos professores do IME que tive contato durante o período em que fiz o doutorado, em particular a Profa. Helena Ávila e os Professores André de Carvalho, Edson de Faria e Albert Fisher. Aos funcionários da secretaria da MAP e da secretaria de pós-graduação do IME.

Ao Prof. Cláudio Pessoa (UFU) com quem tive um maior contato e que me ajudou ao longo da preparação da tese. Aos Professores Luis Fernando (UNIFEI), Clodoaldo Ragazzo (IME), Ronaldo Garcia (UFG) e Orlando Lopes (IME) pelas sugestões e pelas dicas para estudos futuros.

Por fim, agradeço à CAPES pelo apoio financeiro ao longo do doutorado. 


\section{Resumo}

Seja $M$ um conjunto compacto e conexo do $\mathbb{R}^{2}$ que seja a união dos subconjuntos conexos $N$ e $S$. Seja $Z_{\lambda}=\left(X_{\lambda}, Y_{\lambda}\right)$ uma família a um parâmetro de campos vetoriais descontínuos, onde $X_{\lambda}$ está definida em $N$ e $Y_{\lambda}$ em $S$. Ambos os campos $X_{\lambda}$ e $Y_{\lambda}$, assim como as suas dependências em $\lambda$, são suaves i. e. de classe $C^{\infty}$; a descontinuidade acontece na fronteira comum entre $N$ e $S$. O objetivo deste trabalho é estudar as bifurcações que ocorrem em certas famílias de campos vetoriais descontínuos seguindo as convenções de Filippov ${ }^{1}$.

Aplicando o método da regularização, introduzido por Sotomayor e Teixeira e posteriormente aprofundado por Sotomayor e Machado², à família de campos vetoriais descontínuos $Z_{\lambda}$ obtemos uma família de campos vetoriais suaves que é próxima da família descontínua original. Usamos esta técnica de regularização para estudar, por comparação com os resultados clássicos da teoria suave, as bifurcações que ocorrem nas famílias de campos vetoriais descontínuos.

Na literatura há uma lista de bifurcações de codimensão um, no contexto de Filippov, apresentada mais completamente, no artigo de Yu. A. Kuznetsov, A. Gragnani e S. Rinaldi One-Parameter Bifurcations in Planar Filippov Systems, Int. Journal of Bifurcation and Chaos, vol. 13, No. 8: 2157-2188, (2003). Alguns dos casos dessa lista já eram conhecidos por Kozlova, Filippov e Machado.

Neste trabalho nos propomos a estudar as bifurcações de alguns dos casos, apresentados no artigo de Kuznetsov et. al, através do método da regularização dessas famílias.

Nesta Tese consubstanciamos matematicamente a seguinte conclusão: As bifurcações das famílias descontínuas analisadas ficam completamente conhecidas através das bifurcações apresentadas pelas respectivas famílias regularizadas, usando recursos da teoria clássica suave.

Palavras-chave: Campos vetoriais descontínuos, bifurcações, regularização.

\footnotetext{
${ }^{1}$ Differential Equations with discontinuos righthand sides, Kluwer, 1988.

${ }^{2}$ Regularization of Discontinuous Vector Fields, World Scientific Publishing River Edge, 1998.
} 


\section{Abstract}

Let $M$ be a connected and compact set of $\mathbb{R}^{2}$ which is the union of the connected subsets $N$ and $S$. Let $Z_{\lambda}=\left(X_{\lambda}, Y_{\lambda}\right)$ be a one-parameter family of discontinuous vector fields, where $X_{\lambda}$ is defined on $N$ and $Y_{\lambda}$ on $S$. The two fields $X_{\lambda}, Y_{\lambda}$ and their dependences on $\lambda$ are smooths, i. e., are of $C^{\infty}$ class; the discontinuity happens in the common boundary of $N$ and $S$. The objective of this work is to study the bifurcations which occurs in certains families of discontinuous vector fields following the conventions of Filippov ${ }^{3}$.

Applying the regularization method, introduced by Sotomayor and Teixeira ${ }^{4}$, to the family of discontinuous vector fields $Z_{\lambda}$ we obtain a family of regular vector fields which is close to the original family of discontinuous vector fields.

In the literature there is a list of codimension one bifurcation, in the Filippov sense, presented more completely, in the article of Yu. A. Kuznetsov, A. Gragnani e S. Rinaldi One-Parameter Bifurcations in Planar Filippov Systems, Int. Journal of Bifurcation and Chaos, vol. 13, No. 8: 2157-2188, (2003). Some of those cases was already known by Kozlova, Filippov and Machado.

In this work we propose to study the bifurcations of some of those cases, presented in the article of Kuznetsov et. al, by the method of regularization of those families.

In this thesis we justify mathematically the following conclusion: The bifurcations of the analysed discontinuous families are completelly known by the bifurcations contained in the respective regularized families, using the methods of the classical theory of regular vector fields.

Keywords: Discontinuous vector fields, bifurcations, regularization.

\footnotetext{
${ }^{3}$ Differential Equations with discontinuos righthand sides, Kluwer , 1988

${ }^{4}$ Regularization of Discontinuous Vector Fields, World Scientific Publishing River Edge, 1998.
} 


\section{Sumário}

Lista de Abreviaturas $\quad$ viii

Lista de Símbolos $\quad$ ix

1 Introdução 1

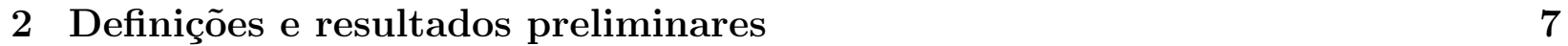

2.1 Campos vetoriais contínuos e descontínuos . . . . . . . . . . . . . . 7

2.2 Regularização de campos vetoriais descontínuos . . . . . . . . . . . . . . 17

2.3 Campos vetoriais lineares e constantes . . . . . . . . . . . . . . . . 31

2.3.1 Eliminação do resto . . . . . . . . . . . . . . . . . . . . 32

2.3.2 Mudança de variáveis . . . . . . . . . . . . . . . . 36

3 Bifurcação foco no bordo - parte I $\quad 41$

3.1 Definição dos casos analisados . . . . . . . . . . . . . . . . . . . . . 41

3.2 Bifurcações de $\mathcal{F}_{1}$ e $\mathcal{F}_{2}$ via regularização . . . . . . . . . . . . . . . . . 56

3.3 Bifurcação de $\mathcal{F}_{1}$ via regularização - continuação . . . . . . . . . . . . . . . 68

3.4 Bifurcação de $\mathcal{F}_{2}$ via regularização . . . . . . . . . . . . . . . . . . . . 85

3.5 Bifurcação de $\mathcal{F}_{4}$ via regularização . . . . . . . . . . . . . . . . . . . 87

3.6 Diagramas de bifurcação de Filippov-Bogdanov-Takens . . . . . . . . . . . . 104

3.7 Exemplo numérico . . . . . . . . . . . . . . . . . . . 109

4 Bifurcação foco no bordo - parte II $\quad 119$

4.1 Bifurcação de $\mathcal{F}_{3}$ via regularização . . . . . . . . . . . . . . . . . . . . . . . 119

4.2 Bifurcação de $\mathcal{F}_{5}$ e $\mathcal{F}_{6}$ via regularização . . . . . . . . . . . . . . . . . . . . . 124

5 Bifurcação Sela no bordo $r 29$

5.1 Bifurcação sela no bordo via regularização . . . . . . . . . . . . . . . . . . . 129 
6 Bifurcação nó no bordo 139

6.1 Bifurcação nó no bordo via regularização . . . . . . . . . . . . . . . . . . . 139

7 Bifurcação tangência dupla externa $\quad 145$

7.1 Bifurcação tangência dupla externa via regularização . . . . . . . . . . . . . 145

8 Exemplo analisado $\quad 165$

8.1 Bifurcação sela e nó no bordo via regularização . . . . . . . . . . . . . 165

9 Conclusões $\quad 173$

$\begin{array}{ll}\text { Glossário } & 174\end{array}$

$\begin{array}{ll}\text { Referências Bibliográficas } & 177\end{array}$ 


\title{
Lista de Abreviaturas
}

\author{
BT Bifurcação de Bogdanov-Takens. \\ CVD Campo Vetorial Descontínuo. \\ CVR Campo Vetorial Regularizado. \\ FBT Bifurcação de Filippov-Bogdanov-Takens. \\ FCD Família de Campos Vetoriais Descontínuos. \\ FCR Família de Campos Vetoriais Regularizados. \\ FH Bifurcação de Filippov-Hopf. \\ FSN Bifurcação de Filippov-sela-nó. \\ SN Bifurcação sela-nó.
}




\section{Lista de símbolos}

$\begin{array}{lll}\text { Símbolo } & \text { descrição } & \text { página } \\ & & \\ & & \\ \mathcal{C}_{Z} & \text { Conjunto de costura } & 22 \\ C^{r} & \text { Aplicações com } r \text { derivadas contínuas } & 19 \\ C^{r}(M) & \text { Aplicações definidas em } M \text { com } r \text { derivadas contínuas } & 19 \\ d f & \text { Derivada de } f & 23 \\ D & \text { Conjunto de descontinuidade } & 21 \\ \mathcal{D}_{Z} & \text { Conjunto de deslizamento } & 22 \\ \operatorname{det} & \text { Determinante } & 19 \\ \operatorname{det}(X, Y)(p) & \text { Determinante dos campos } X \text { e } Y & 23 \\ \mathcal{E}_{Z} & \text { Conjunto de escape } & 22 \\ F & \text { Função real que define } M & 21 \\ F_{Z}(q) & \text { Campo vetorial de Filippov } & 22 \\ G & \text { Função que distingue os casos } \mathcal{F}_{1}, \mathcal{F}_{2} \text { e } \mathcal{F}_{4} & 52 \\ l_{1} & \text { Primeiro número de Lyapunov } & 69 \\ M & \text { Conjunto compacto e conexo do plano } & 21 \\ N & \text { Conjunto Norte de } M & 21 \\ \mathbb{N} & \text { Conjunto dos números naturais, incluindo o zero } & \\ \mathbb{R} & \text { Conjunto dos números reais } & 21 \\ R_{\varepsilon} & \text { Região de descontinuidade } & 58 \\ S & \text { Conjunto Sul de } M & 21 \\ \operatorname{tr} & \text { Traço. } & \\ \mathbb{Z} & \text { Conjunto dos números inteiros. } & \\ Z_{\lambda} & \text { Família a um parâmetro de campos vetoriais descontínuos } & 20 \\ Z_{\lambda, R} & \text { Família de campos vetoriais regularizados } & \\ & & \\ & & \\ & & \\ & & \\ & & \\ & & \end{array}$


$\mathfrak{X}^{r}(M)$ Conjunto de campos vetoriais de classe $C^{r}$ definidos em $M \quad 19$

$\Delta \quad$ Discriminante $\quad 52$

$\begin{array}{lll}\varepsilon & \text { Parâmetro de regularização } & 15\end{array}$

$\begin{array}{lll}\lambda & \text { Parâmetro real } & 16\end{array}$

$\pi \quad$ Função de primeiro retorno de Poincaré 28

$\varphi_{\varepsilon}(x) \quad$ Função de transição 30

$\Omega^{r}(M)$ Conjunto dos $C^{r}$ campos vetoriais descontínuos em $M \quad 19$. 


\section{Capítulo 1}

\section{Introdução}

Um campo vetorial de classe $C^{r}, r \geq 1$, definido em um aberto $U$ do plano, $\mathbb{R}^{2}$, é uma aplicação $X: U \rightarrow \mathbb{R}^{2}$ de classe $C^{r}$. Ao campo vetorial $X$ associamos a equação diferencial

$$
p^{\prime}=X(p)
$$

As soluções desta equação são funções diferenciáveis $\varphi: I \subset \mathbb{R} \rightarrow U$ que satisfazem

$$
\frac{d \varphi}{d t}(t)=X(\varphi(t))
$$

para todo $t \in I$, e são chamadas de trajetórias, órbitas ou curvas integrais do campo vetorial X, ou da equação (1.1). A Teoria Qualitativa das Equações Diferenciais Ordinárias, veja [S2], cujo fundador foi Poincaré no final do século XIX, pode ser resumida como sendo o estudo qualitativo das órbitas do campo vetorial $X$ associado à equação diferencial ordinária (1.1). A importância desta Teoria se deve ao fato de obtermos resultados precisos e profundos sobre a equação diferencial sem precisarmos resolvê-la explicitamente. A teoria dos Sistemas Dinâmicos foi desenvolvida tendo como ponto de partida a Teoria Qualitativa das Equações Diferenciais Ordinárias.

Dizemos que um campo vetorial $Z: M \rightarrow \mathbb{R}^{2}$ é descontínuo se $M$ for dividido em subconjuntos, não vazios, $N$ e $S$ que têm um conjunto em comum, chamado de conjunto de descontinuidade e que denotamos por $D$, no qual $\left.Z\right|_{N}$ difere de $\left.Z\right|_{S}$. Denotamos o campo vetorial descontínuo $Z$, definido em $M$, por $Z=(X, Y)$ onde $X=\left.Z\right|_{N}$ e $Y=\left.Z\right|_{S}$. A teoria dos sistemas dinâmicos descontínuos está em amplo desenvolvimento devido a sua aplicabilidade em diversas áreas tais como engenharia mecânica, engenharia de controle, engenharia elétrica, biologia e física. Para exemplos nessas áreas recomendamos [AVK], [KGR]

e $[\mathrm{BBCK}]$. Além das aplicações, a compreensão matemática de tais sistemas é por si só um 
incentivo para a sua análise, e usaremos esse argumento como a motivação central deste trabalho.

A teoria dos sistemas dinâmicos descontínuos tem tido avanços desde a época de A. A. Andronov, ou seja, desde a década de 1940. Nesse início as análises de sistemas descontínuos eram todas feitas através de exemplos particulares de sistemas descontínuos na engenharia elétrica ou mecânica, veja $[\mathrm{AVK}]$. Um dos problemas iniciais da teoria era o de definir de forma precisa o que viria a ser uma órbita, uma vez que tal definição só é possível na presença de continuidade e das condições de Lipschitz. Assim, seria necessário uma teoria que resolvesse esse problema, e foi Filippov quem sistematizou a análise desse problema das órbitas, veja $[\mathrm{F}]$. A ideia de Filippov foi inicialmente definir regras, chamadas de "convenções de Filippov", para a transição de órbitas entre as regiões $N$ e $S$ passando pelo conjunto de descontinuidade, e também para a permanência das órbitas em $D$. Em suma, essas convenções são três, uma para cada padrão de incidência em $D$ dos campos $X$ e $Y$, para a apresentação dessas convenções veja [ST], [SM] ou o capítulo dois deste trabalho. Além disto, Filippov apresentou um campo vetorial no conjunto de descontinuidade que dá um critério de escolha, eliminando o fato de, nesse conjunto, o campo vetorial descontínuo ser bivaluado. Esse campo é chamado de campo vetorial de Filippov, apesar de não ter unicidade das órbitas, esse campo vetorial é um modelo útil nas aplicações e se adapta bem para o estudo da Estabilidade Estrutural para os campos descontínuos, e consequentemente, nas bifurcações de tais campos.

Na década de 1990 com o artigo [ST], Sotomayor e Teixeira formalizaram matematicamente o método da regularização de campos vetoriais descontínuos. A regularização de um campo vetorial descontínuo é uma família de campos vetoriais suaves. Assim, a partir desse artigo podia-se então analisar os resultados sobre sistemas dinâmicos descontínuos através da análise de sistemas dinâmicos suaves muito próximos aos sistemas iniciais. Nosso trabalho baseia-se nesse princípio, ou seja, confirmar alguns resultados sobre certos sistemas dinâmicos descontínuos através da comparação com a análise dos respectivos sistemas regularizados.

Vamos, de forma resumida, apresentar o método da regularização de Sotomayor e Teixeira. Seja $M \subset \mathbb{R}^{2}$ compacto e conexo, supomos, sem perda de generalidade, que existe uma função suave $F: M \rightarrow \mathbb{R}$ tal que 0 é um valor regular de $F \operatorname{com} D=F^{-1}(0)$. Vamos supor que $D$ tenha uma componente conexa e que $M \backslash D$ tenha duas componentes conexas dadas por

$$
\begin{aligned}
& N=\left\{(x, y) \in \mathbb{R}^{2}: F(x, y) \geq 0\right\} \\
& S=\left\{(x, y) \in \mathbb{R}^{2}: F(x, y) \leq 0\right\} .
\end{aligned}
$$


Assim, se $Z=(X, Y)$ é um campo vetorial descontínuo em $M$ temos que $X$ está definido em $N$ e $Y$ em $S$. Uma vez definido um campo vetorial descontínuo, passamos à sua regularização e para isso precisamos de uma função auxiliar que é chamada de função de transição. Uma função $\varphi: \mathbb{R} \rightarrow \mathbb{R}$ de classe $C^{\infty}$ é uma função de transição se

$$
\varphi(x)= \begin{cases}0, & x \leq-1 \\ 1, & x \geq 1\end{cases}
$$

e $\varphi^{\prime}(x)>0$ em $(-1,1)$. Dado $\varepsilon>0$, a regularização, ou simplesmente a família regularizada, de um campo vetorial descontínuo $Z=(X, Y)$ em $M$, é definida pela família a um parâmetro de campos vetoriais regulares, ou suaves, $Z_{\varepsilon}$, definida por

$$
Z_{\varepsilon}(x, y)=\left(1-\varphi_{\varepsilon}(F(x, y))\right) Y(x, y)+\varphi_{\varepsilon}(F(x, y)) X(x, y)
$$

onde $(x, y) \in M$ e $\varphi_{\varepsilon}(F(x, y))=\varphi\left(\frac{F(x, y)}{\varepsilon}\right)$. Dizemos que o parâmetro $\varepsilon$ é o parâmetro de regularização.

Da definição da função de transição e da regularização de $Z=(X, Y)$ temos a existência de uma região em $M$, chamada de região de regularização, e denotada por $R_{\varepsilon}$, onde o campo $Z_{\varepsilon}$, para um $\varepsilon$ fixado, é dado por uma "soma" com peso $\varphi_{\varepsilon} \operatorname{dos}$ campos $X$ e $Y$. Essa soma faz com que o campo resultante seja suave, ou seja, é em $R_{\varepsilon}$ que ocorre o processo de regularização, propriamente dito, do campo descontínuo, por isso a importância desse conjunto. A região de regularização é exatamente o suporte compacto da função de transição aplicada em $F(M)$, ou seja, é o conjunto $R_{\varepsilon}=\{(x, y) \in M: F(x, y) \in(-\varepsilon, \varepsilon)\}$. Fora de $R_{\varepsilon}$ temos que $Z_{\varepsilon}$ coincide com o campo $X$ em $N$ e com o campo $Y$ em $S$.

Relembramos a seguir o conceito de Estabilidade Estrutural de campos vetoriais suaves, desenvolvido inicialmente por Andronov, Pontrjaguin e posteriormente por Peixoto. Dois campos suaves $X$ e $Y$ são ditos topologicamente equivalentes se existe um homeomorfismo que leva órbitas de $X$ em órbitas de $Y$, preservando a orientação. Diz-se que um campo vetorial suave $X$ é estruturalmente estável se existir uma vizinhança ao redor de $X$ tal que qualquer campo vetorial nessa vizinhança é topologicamente equivalente a $X$.

Os campos vetoriais, de classe $C^{r}, r \geq 1$, de Andronov-Pontrjaguin-Peixoto são todos estruturalmente estáveis e, além disso, Peixoto mostrou que tais campos formam um conjunto aberto e denso no espaço de todos os campos vetoriais suaves de classe $C^{r}$.

Em [ST], Sotomayor e Teixeira consideraram campos vetoriais descontínuos definidos na 
esfera de dimensão dois, aplicaram o método da regularização nesses campos e estabeleceram condições nos campos descontínuos para que os campos vetoriais regularizados sejam do tipo Andronov-Pontrjaguin-Peixoto, para valores pequenos do parâmetro de regularização. Ainda nessa linha de estudo, em [M] e [SM] foi considerado o caso em que $M$ é um subconjunto compacto e conexo do plano com bordo suave.

Em contrapartida ao conceito de estabilidade estrutural há o conceito de bifurcação, ou seja, a quebra da estabilidade estrutural. Assim, se temos uma família a um parâmetro real, $\lambda$, de campos vetoriais suaves, $X_{\lambda}$, dizemos que ocorre uma bifurcação no parâmetro $\lambda_{0}$, chamado de parâmetro de bifurcação, se para qualquer vizinhança de $\lambda_{0}$, existem valores $\lambda$, tais que $X_{\lambda}$ não é topologicamente equivalente ao campo $X_{\lambda_{0}}$. Há vários tipos de bifurcações que ocorrem genericamente para famílias a um parâmetro de campos vetoriais definidos no plano, veja por exemplo [K], [GH], [S1], [S3].

Bautin e Leontovich no livro "Métodos e Técnicas de Análise Qualitativa de Sistemas Dinâmicos no Plano" ${ }^{1}$ do ano de 1976, consideraram as bifurcações locais que ocorrem em sistemas descontínuos do plano, porém eles apresentaram uma classificação incompleta. Kozlova e Filippov, veja $[\mathrm{F}]$ e as referências contidas neste livro, classificaram as singularidades dos sistemas descontínuos no plano e identificaram as bifurcações locais de codimensão um decorrentes de singularidades, porém as bifurcações que tratam de ciclos contendo partes do conjunto de descontinuidade não foram consideradas. Mais recentemente, em [KGR], há uma lista que sintetiza todas as bifurcações de codimensão um para sistemas dinâmicos descontínuos no plano consideradas por Kozlova e Filippov, além das bifurcações globais, que tratam de ciclos que têm partes no conjunto de descontinuidade.

Nos artigos [ST] e [SM] temos o estudo da teoria da estabilidade estrutural dos campos vetoriais descontínuos através do método da regularização. Neste trabalho daremos continuidade ao estudo dos campos vetoriais descontínuos no sentido das bifurcações. Um modo de se efetuar este estudo é explicar pelo método da regularização as bifurcações existentes na lista das bifurcações de codimensão um no plano. Outro modo seria o de estudar as bifurcações que ocorrem nas regularizações dos campos vetoriais descontínuos que não satisfazem as condições descritas em [ST] ou [SM], dependendo do conjunto $M$ ter ou não bordo suave. Preferimos estudar as bifurcações através do primeiro modo descrito.

Assim, o objetivo desta tese é o estudo das bifurcações para certas famílias a um parâmetro de campos vetoriais descontínuos, $Z_{\lambda}=\left(X_{\lambda}, Y_{\lambda}\right)$, através da comparação das bifurcações das respectivas famílias regularizadas. Da lista das bifurcações de codimensão um de Kozlova-

\footnotetext{
${ }^{1}$ Aparentemente esse livro só está disponível no idioma original, o russo, cujo título original é "Metody i Priemy Kachestvennogo Issledovaniia Dinamicheskikh Sistem Na Ploskosti".
} 
Filippov-Kuznetsov-Gragnani-Rinaldi consideramos as principais descritas por Kozlova e Filippov, que ocorrem da colisão de singularidades com o conjunto de descontinuidade dentre as quais destacamos a colisão do foco que gera três tipos distintos de bifurcação de codimensão um e uma bifurcação de codimensão dois. Além destas, analisaremos a bifurcação que Filippov chama de "focos fundidos", que trata do caso decorrente de duas tangências quadráticas externas, uma da família $X_{\lambda}$ e outra de $Y_{\lambda}$, que colidem quando o parâmetro se anula. Analisaremos ainda dois casos sobre a bifurcação global dada pela tangência de um ciclo limite, em um caso esse ciclo é atrator e no outro repuslor, de $X_{\lambda}$ com o conjunto de descontinuidade.

Para a regularização de famílias descontínuas iremos utilizar a função

$$
\varphi(x)=\frac{1}{2}+\frac{x}{2 \sqrt{x^{2}+1}} .
$$

Não se trata de uma função de transição, no sentido estrito definido acima, pois enquanto a função de transição tem suporte compacto para sua derivada, esta função não possui esta propriedade. Porém, para nossos propósitos ela é satisfatória pois está tão próxima de uma função de transição quanto quisermos, bastando para isso tomarmos valores cada vez menores de $\varepsilon>0$. Além disso, essa função é uma das mais simples para efetuarmos os cálculos das bifurcações. Também pode-se provar que a regularização, com relação a esta função, dos campos descontínuos da classe de Machado e Sotomayor também são de Andronov, Pontrjaguin e Peixoto. Este resultado está em fase de redação.

Agora apresentamos um resumo de cada capítulo da tese.

No segundo capítulo apresentamos alguns resultados da teoria de campos vetoriais regulares e os conceitos básicos da teoria dos sistemas dinâmicos descontínuos e da regularização desses campos. Além disso, apresentamos alguns resultados básicos de regularização, usando a função $\varphi(x)=1 / 2+x /\left(2 \sqrt{x^{2}+1}\right)$. Após isto, apresentamos uma última seção que trata de dois assuntos em particular. O primeiro é uma verificação de que podemos utilizar uma família descontínua cujo campo em $N$ é dado pela parte linear apenas, sendo que o resto com termos de ordem maior ou igual a dois se anula com o parâmetro de regularização. O segundo assunto é uma mudança de variáveis que apresentamos para simplificar a expressão do campo definido em $S$.

No capítulo três começamos a analisar as bifurcações das famílias vetoriais descontínuas. Consideramos a presença de um foco repulsor, para valores negativos e pequenos do parâmetro, da família definida em $N$, que colide com o conjunto de descontinudiade quando o parâmetro se anula. Analisamos seis subcasos, três dos quais estão contidos neste capítulo sendo que 
em um deles ocorre uma bifurcação de codimensão dois, a saber, a bifurcação de BogdanovTakens. Esse caso de codimensão dois não foi apresentado na lista de bifurcações de FilippovKozlova ou na lista geral contida em [KGR]. Apresentamos os subcasos restantes de foco no bordo no capítulo quatro.

No quinto capítulo estudamos o caso em que a família definida em $N$ tem uma sela que colide com o conjunto de descontinuidade. Temos dois subcasos nesse capítulo, e analisamos uma família de campos descontínuos que engloba esses dois casos. O resultado obtido em ambos os casos desse capítulo é que ocorre uma bifurcação do tipo sela-nó.

No sexto capítulo obtemos um resultado análogo ao do capítulo quinto, ou seja, ocorre uma bifurcação do tipo sela-nó. Neste capítulo o campo vetorial descontínuo possui um nó em $N$ que colide com o conjunto de descontinuidade quando o parâmetro se anula.

No penúltimo capítulo analisamos o caso denominado por Filippov de "foco fundido". Trata-se da colisão de duas tangências quadráticas externas quando o parâmetro se anula, e para valores positivos e pequenos do parâmetro há o aparecimento de um ciclo limite. O artigo [CGP] apresenta o estudo da bifurcação desse caso, e eles usam a teoria de Blowup generalizado para obter, para o campo vetorial descontínuo, um número equivalente ao primeiro número de Lyapunov no caso regular. O que fazemos nesse capítulo é estudar a regularização de uma família descontínua que tenha esse número, descrito em [CGP], negativo, ou seja, partimos de um caso onde o campo descontínuo tem um ciclo limite estável. O resultado obtido foi que, sob hipóteses adicionais nos coeficientes das famílias de campos descontínuos, ocorre uma bifurcação de Hopf onde o primeiro número de Lyapunov é negativo.

No capítulo oito temos o estudo de um exemplo dado em [KGR] onde temos a presença de uma sela e um nó, ambos de Filippov, que colidem quando o parâmetro se anula. A regularização deste caso possui uma bifurcação sela-nó. 


\section{Capítulo 2}

\section{Definições e resultados preliminares}

Este capítulo serve de material de referência para a leitura do restante do trabalho. Introduziremos os conceitos básicos e alguns resultados conhecidos sobre a teoria dos campos vetoriais contínuos e descontínuos. Apresentamos ainda o método da regularização dos campos vetoriais descontínuos conforme Sotomayor e Teixeira em [ST], tal método se torna útil para compararmos um campo vetorial descontínuo com uma família a um parâmetro real de campos vetoriais contínuos.

\subsection{Campos vetoriais contínuos e descontínuos}

Um campo vetorial contínuo, ou, um campo vetorial ou, simplesmente, um campo $X$ suave de classe $C^{r}, r \geq 1$, definido em um aberto $U$ qualquer do plano $\mathbb{R}^{2}$ é uma aplicação de classe $C^{r}$ definida em $U$ com valores em $\mathbb{R}^{2}$, ou seja, $X: U \rightarrow \mathbb{R}^{2}$. A este campo vetorial associamos a seguinte equação diferencial ordinária

$$
x^{\prime}=X(x)
$$

onde $x \in U \subset \mathbb{R}^{2}$. Assim, as soluções da equação diferencial ordinária (2.1) são chamadas de órbitas ou trajetórias de $X$.

O conjunto dos campos vetoriais de classe $C^{r}$ definidos em $U$ será denotado por $\mathfrak{X}^{r}(U)$.

Um ponto $x \in U$ é dito ponto singular, ou simplesmente uma singularidade do campo $X$ se $X(x)=0$. Ele é dito ponto regular, ou não singular, de $X$ se $X(x) \neq 0$.

Usaremos alguns resultados clássicos da teoria qualitativa das equações diferenciais ordinárias, para tanto vamos enunciar tais resultados, indicando as referências para as suas demonstrações.

O primeiro resultado que apresentamos nos dá elementos para garantir quando um campo vetorial é $C^{r}$-conjugado a um campo constante. Nesse resultado usamos o conceito de seção 
transversal local que definimos agora. Uma aplicação diferenciável $f: A \subseteq \mathbb{R} \rightarrow U$ de classe $C^{r}$ chama-se seção tranversal local de $X \in \mathfrak{X}^{r}(U)$ quando, para todo $a \in A, D f(a)(\mathbb{R})$ e $X(f(a))$ geram o espaço $\mathbb{R}^{2}$.

Teorema 1 (Fluxo tubular). Seja p um ponto não singular de $X$ e $f: A \rightarrow \Sigma$ uma seção tranversal local de $X$ de classe $C^{r}$ com $f(0)=p$. Então existe uma vizinhança $V$ de $p$ em $U$ e um difeomorfismo $h: V \rightarrow(-\varepsilon, \varepsilon) \times B$ de classe $C^{r}$, onde $\varepsilon>0$ e $B$ é uma bola aberta em $\mathbb{R}$ de centro na origem $0=f^{-1}(p)$ tal que

a) $h(\Sigma \cap V)=\{0\} \times B$;

b) h é uma $C^{r}$-conjugação entre $\left.X\right|_{V}$ e o campo constante $Y:(-\varepsilon, \varepsilon) \times B \rightarrow \mathbb{R}^{2}, Y=$ $(1,0) \in \mathbb{R}^{2}$.

Demonstração. Veja [S2].

O segundo resultado descreve o comportamento assintótico das soluções e a estrutura de seus conjuntos limites. Assim, seja $\varphi(t)=\varphi(t, p)$ a solução, ou curva integral, de $X$ passando pelo ponto $p$ definida no seu intervalo máximo $I_{p}=\left(\omega_{-}(p), \infty\right)$. O conjunto $\omega$-limite de $p$ é dado pelo conjunto

$$
\omega(p)=\left\{q \in U: \exists\left(t_{n}\right)_{n \in \mathbb{N}}, t_{n} \rightarrow \infty, \text { tal que } \varphi\left(t_{n}\right) \rightarrow q \text {, quando } n \rightarrow \infty\right\}
$$

Analogamente, se $\omega_{-}(p)=-\infty$, definimos o conjunto $\alpha$-limite de $p$ por

$$
\alpha(p)=\left\{q \in U: \exists\left(t_{n}\right)_{n \in \mathbb{N}}, t_{n} \rightarrow-\infty, \text { tal que } \varphi\left(t_{n}\right) \rightarrow q, \text { quando } n \rightarrow \infty\right\}
$$

Teorema 2 (Poincaré-Bendixson). Seja $\varphi(t)=\varphi(t, p)$ uma curva integral de $X$, definida para todo $t \geq 0$, tal que a semiórbita positiva por $p$, dada por $\gamma_{p}^{+}=\{\varphi(t, p): t \geq 0\}$ esteja contida em um subconjunto compacto $K$ de $U$. Suponha que o campo $X$ possua um número finito de singularidades em $\omega(p)$. Têm-se as seguintes alternativas

a) se $\omega(p)$ contém somente pontos regulares, então $\omega(p)$ é uma órbita periódica.

b) se $\omega(p)$ contém pontos regulares e singulares, então $\omega(p)$ consiste de um conjunto de órbitas, cada uma das quais tende a um desses pontos singulares quando $t \rightarrow \pm \infty$.

c) se $\omega(p)$ não contém pontos regulares, então $\omega(p)$ é um ponto singular.

Demonstração. Veja [S2]. 
Utilizaremos ainda a transformação de primeiro retorno, ou de Poincaré, associada a uma órbita fechada de $X$, para sua definição formal e resultados relacionados à transformação de Poincaré recomendamos [A], [S2] ou [S1].

Agora que temos alguns resultados básicos da teoria qualitativa das equações diferenciais, vamos apresentar os conceitos e resultados básicos da teoria dos campos vetoriais descontínuos definidos em um conjunto compacto do plano.

Um campo vetorial descontínuo definido em um conjunto compacto e conexo $M$ do plano é determinado por dois campos vetoriais e uma aplicação real suave que define $M$.

Definição 1. Sejam $X, Y \in \mathfrak{X}^{r}(M)$ e $F: M \rightarrow \mathbb{R}$ uma aplicação real suave definida em $M$ tal que 0 é seu valor regular. Vamos supor que o conjunto $D=F^{-1}(0)$ é compacto e conexo tal que $M-D$ tem exatamente duas componentes conexas que são $S=F^{-1}(-\infty, 0]$ e $N=F^{-1}[0, \infty)$. Assim, o campo vetorial descontínuo $Z=(X, Y)$ definido em $M$ é dado por

$$
Z(q)= \begin{cases}X(q), & F(q) \geq 0 \\ Y(q), & F(q) \leq 0 .\end{cases}
$$

O conjunto dos campos vetoriais descontínuos definidos em $M$ será denotado por $\Omega^{r}(M)$. Além disso, o conjunto $D$ será chamado de conjunto de descontinuidade.

Para fixar notação, ao longo deste trabalho iremos considerar a família a um parâmetro, $\lambda \in \mathbb{R}$, de campos vetoriais descontínuos $Z_{\lambda}=\left(X_{\lambda}, Y_{\lambda}\right)$ definida em $M$, onde $M$ é um conjunto compacto e conexo dado pela função suave $F: M \rightarrow \mathbb{R}$, e onde $D=F^{-1}(0)$ é o conjunto de descontinuidade, $N$ e $S$ são definidos como na definição anterior. Portanto, de agora em diante, iremos trabalhar com essa notação, sendo que os campos vetoriais descontínuos são definidos como acima.

Filippov, veja $[\mathrm{F}]$, definiu três regras para a transição das órbitas de um campo descontínuo entre as regiões $S$ e $N$, cruzando ou atingindo o conjunto $D$ segundo diversos padrões. A classificação destes padrões e as respectivas regras são denominadas de convenções de Filippov.

Para definir as convenções de Filippov usaremos a seguinte notação: $X F(p)$ significa a derivada de $F$ na direção $X$. Assim, se escrevemos localmente $X(p)=(a(p), b(p))$, para um ponto qualquer $p \in M$, então temos que

$$
X F(p)=\left\langle(a(p), b(p)),\left(\frac{\partial F}{\partial x}(p), \frac{\partial F}{\partial y}(p)\right)\right\rangle=a(p) \frac{\partial F}{\partial x}(p)+b(p) \frac{\partial F}{\partial y}(p),
$$

onde $<,>$ é o produto interno usual do $\mathbb{R}^{2}$. 
Definição 2. As convenções de Filippov são definidas por:

a) Um conjunto $\mathcal{C}_{Z} \subseteq D$ é dito ser de costura, se para todo $p \in \mathcal{C}_{Z}$ tivermos que $X F(p) Y F(p)>0$.

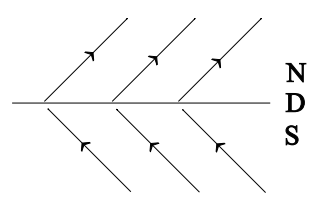

figura 1: Conjunto de costura

b) Um conjunto $\mathcal{E}_{Z} \subseteq D$ é dito ser de escape, se para todo $p \in \mathcal{E}_{Z}$ tivermos que $X F(p)<0$ e $Y F(p)>0$.

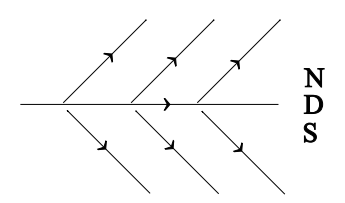

figura 2: Conjunto de escape

c) um conjunto $\mathcal{D}_{Z} \subseteq D$ é dito ser de deslizamento, se para todo $p \in \mathcal{D}_{Z}$ tivermos que $X F(p)>0$ e $Y F(p)<0$.

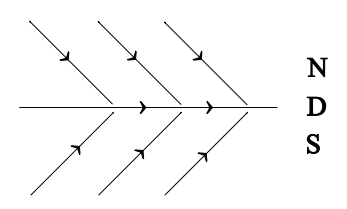

figura 3: Conjunto de deslizamento

Nos conjuntos de deslizamento ou de escape define-se um campo vetorial auxiliar chamado de campo de Filippov.

Definição 3. Sejam $Z=(X, Y) \in \Omega^{r}(M), D$ o conjunto de descontinuidade e $p \in D$ um ponto de escape ou de deslizamento. O campo de Filippov no ponto $p$, denotado por $F_{Z}(p)$, é o único vetor tangente a $D$ em $p$ contido no cone gerado por $X(p)$ e $Y(p)$.

A próxima figura apresenta um exemplo do campo de filippov calculado em um ponto $p \in \mathcal{D}_{Z}$. 


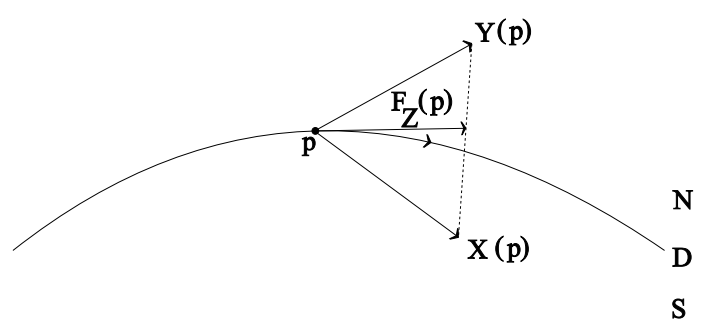

figura 4: Campo de Filippov em $p$

Um modo de calcularmos explicitamente o campo de Filippov em um ponto é apresentado em $[\mathrm{M}]$. Para isso, escrevemos localmente $X(p)$ por $(a(p), b(p))$ e $Y(p)$ por $(c(p), d(p))$. Assim, o campo de Filippov no ponto $p$ tem a seguinte expressão

$$
F_{Z}(p)=\left(\frac{a(p) d(p)-b(p) c(p)}{d(p)-b(p)}, 0\right) .
$$

Usando a notação da expressão do campo de Filippov, ou seja, (2.4) definimos suas singularidades.

Definição 4. Um ponto $p \in D$ é uma singularidade do campo de Filippov se estiver contido no conjunto de definição de $F_{Z}$, quer seja um conjunto de escape ou deslizamento, e deve satisfazer $a(p) d(p)-b(p) c(p)=0$.

Os diferentes tipos de singularidades de $F_{Z}$ são dados pela definição seguinte, onde usaremos a seguinte notação

$$
\operatorname{det}(X, Y)(p)=\operatorname{det}\left(\begin{array}{ll}
a(p) & b(p) \\
c(p) & d(p)
\end{array}\right)=a(p) d(p)-b(p) c(p) .
$$

Definição 5. Dizemos que uma singularidade $p$ do campo de Filippov é hiperbólica se $d\left(\left.\operatorname{det}(X, Y)\right|_{D}\right)(p) \neq 0$.

Definição 6. Se $p$ é uma singularidade hiperbólica de $F_{Z}$, dizemos que

i) p é uma sela de Filippov, ou simplesmente uma sela de $F_{Z}$, se satisfaz uma das condições seguintes

i.1) $p$ é um ponto do conjunto de deslizamento e é uma singularidade repulsora de $F_{Z}$, ou seja, $d\left(\left.\operatorname{det}(X, Y)\right|_{D}\right)(p)>0$ 


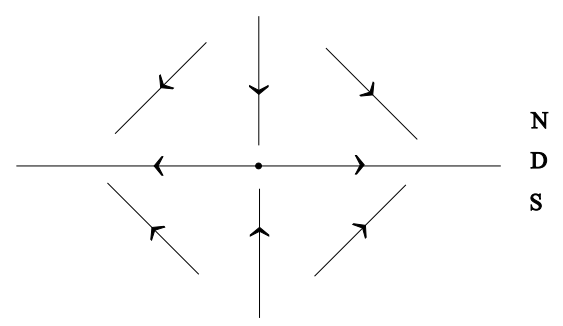

figura 5: Sela de Filippov

i.2) $p$ é um ponto do conjunto de escape e é uma singularidade atratora de $F_{Z}$, ou seja, $d\left(\left.\operatorname{det}(X, Y)\right|_{D}\right)(p)<0$.

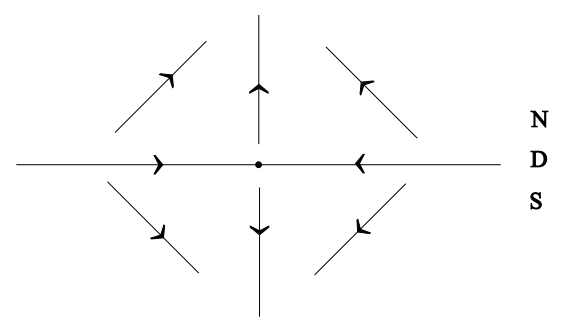

figura 6: Sela de Filippov

ii) p é um nó de Filippov, ou simplesmente um nó de $F_{Z}$, se satisfaz uma das condições seguintes

ii.1) $p$ é um ponto do conjunto de deslizamento e é uma singularidade atratora de $F_{Z}$, ou seja, $d\left(\left.\operatorname{det}(X, Y)\right|_{D}\right)(p)<0$, neste caso o nó é dito atrator

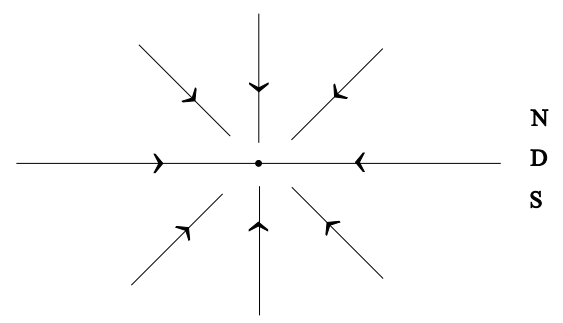

figura 7: Nó atrator de Filippov

ii.2) $p$ é um ponto do conjunto de escape e é uma singularidade repulsora de $F_{Z}$, ou seja, $d\left(\left.\operatorname{det}(X, Y)\right|_{D}\right)(p)>0$, neste caso o nó é repulsor. 


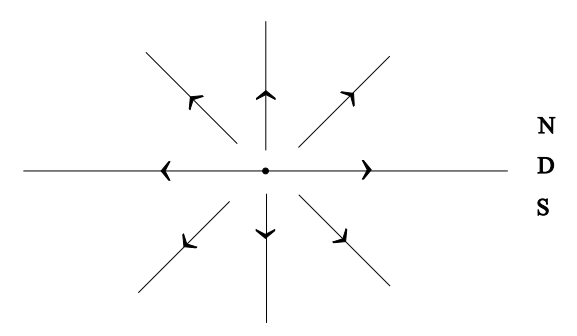

figura 8: Nó repulsor de Filippov

Uma vez definido os pontos que são singularidades do campo de Filippov, temos os pontos onde falham essa definição.

Definição 7. Um ponto $p \in D$ é dito $D$-regular de $Z$ se satisfaz uma das seguintes condições

a) $p$ é um ponto de costura,

b) $p$ é um ponto de deslizamento ou de escape e não é uma singularidade do campo de Filippov, ou seja, $\operatorname{det}(X, Y)(p) \neq 0$.

Outra caracterização de pontos em $D$ são as tangências de órbitas dos campos $X$ ou $Y$ em pontos do conjunto de descontinuidade. Neste trabalho trataremos, no capítulo entitulado "Duas tangências externas", apenas com as tangências quadráticas ao conjunto de descontinuidade.

Definição 8. Um ponto $p \in D$ é dito uma $D$-dobra de $Z$ se for um ponto de tangência quadrática do campo $X$ com $D$, ou do campo $Y$ com $D$. Dizemos que uma $D$-dobra de $Z$ é

a) externa se for um ponto de tangência quadrática externa do campo $X$ com $D$, ou seja, se $Y F(p) \neq 0, X F(p)=0$ e $X^{2} F(p)<0$. Definimos analogamente uma $D$-dobra de $Z$ externa que seja tangência do campo $Y$ ao conjunto $D$. 


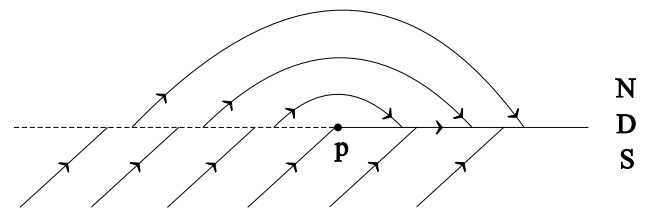

figura 9: $D$-dobra externa

b) interna se for um ponto de tangência quadrática interna do campo $X \operatorname{com} D$, ou seja, se $Y F(p) \neq 0, X F(p)=0$ e $X^{2} F(p)>0$. Definimos analogamente uma $D$-dobra de $Z$ interna que seja tangência do campo $Y$ ao conjunto $D$.

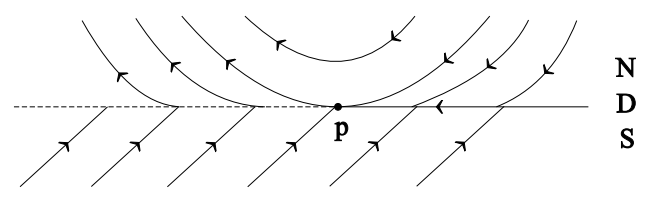

figura 10: $D$-dobra interna

Seguindo $[\mathrm{M}]$ e $[\mathrm{SM}]$, vamos apresentar a definição das órbitas periódicas em um campo vetorial descontínuo.

Definição 9. Seja $\gamma$ uma curva em $M$ composta por arcos regulares de trajetórias de $X$ em $N$, e/ou de trajetórias de $Y$ em $S$, e/ou de trajetórias de $F_{Z}$ em $D$. Nessas condições, dizemos que $\gamma$ é uma politrajetória de $Z$ se satisfizer

i) $\gamma$ contém arcos de trajetória de pelo menos dois entre os campos vetoriais $X, Y$ e $F_{Z}$, ou é formado por um arco de $F_{Z}$;

ii) a transição de arcos de trajetória de $X$ para arcos de trajetória de $Y$ é feita em pontos de costura;

iii) a transição de arcos de trajetória de $X$, ou de $Y$, para arcos de trajetória de $F_{Z}$ é feita através de $D$-dobras, ou de pontos regulares do arco de escape, ou do arco deslizante, respeitando-se o sentido dos arcos de trajetória.

A próxima figura apresenta um exemplo de uma politrajetória $\gamma$ que contém um arco de trajetória de $F_{Z}$ que consiste de pontos de escape.

Agora apresentamos uma caracterização de órbitas fechadas, ou seja, de politrajetórias fechadas de $Z$. 


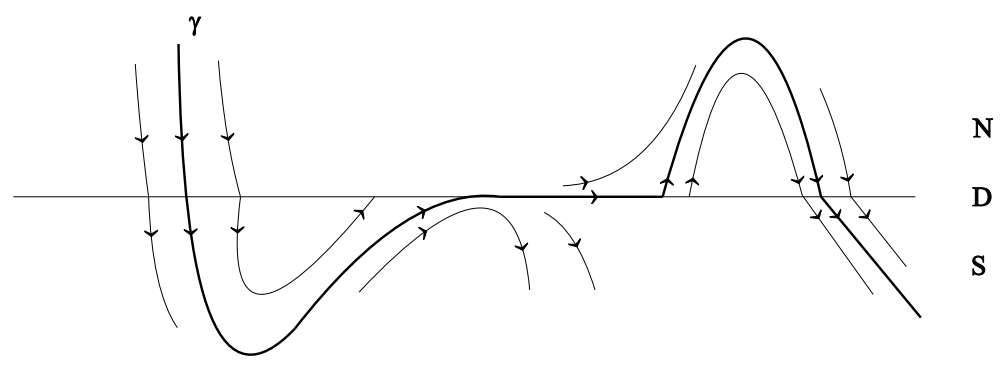

figura 11: politrajetória

Definição 10. Seja $\gamma$ uma politrajetória fechada de $Z$. Dizemos que

a) $\gamma$ é uma politrajetória fechada do tipo I se $\gamma$ encontra $D$ apenas em pontos de costura.

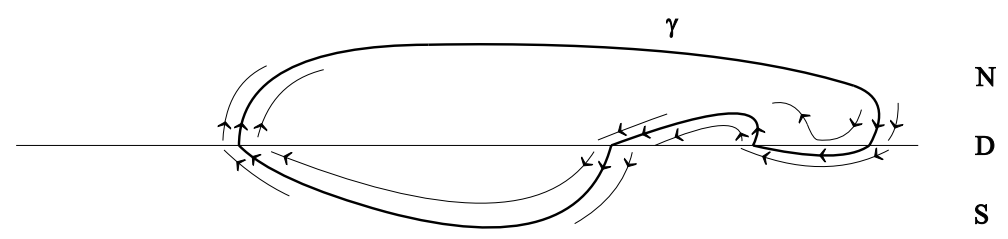

figura 12: politrajetória fechada do tipo I

b) $\gamma$ é uma politrajetória fechada do tipo II se $\gamma=D$.

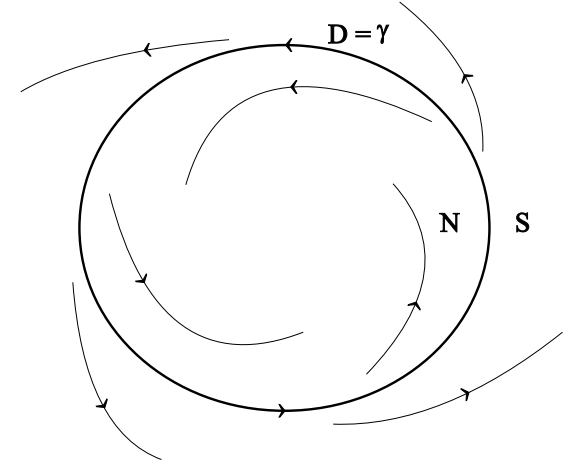

figura 13: politrajetória fechada do tipo II

c) $\gamma$ é uma politrajetória fechada do tipo III se $\gamma$ contém, pelo menos, uma $D$-dobra de $Z$. 


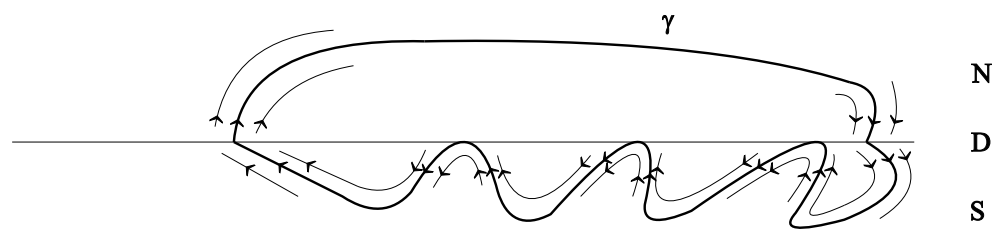

figura 14: politrajetória fechada do tipo III

Obs.: Note que o exemplo da figura 13 para uma politrajetória do tipo II está correto, no sentido que $D$ satisfaz o fato de dividir $M$ em exatamente dois conjuntos conexos.

Agora definimos a transformação de Poincaré, ou aplicação de primeiro retorno, em uma politrajetória do tipo I.

Definição 11. Dada uma politrajetória fechada $\gamma$ do tipo I, com $\gamma=\gamma_{0} \cup \gamma_{1} \cup \cdots \cup \gamma_{n}$ onde $\gamma_{2 j}$ são arcos de trajetória de $X$ em $N$ e $\gamma_{2 j+1}$ são arcos de trajetória de $Y$ em $S$, para $j=0,1, \ldots, \frac{n-1}{2}$. Para cada $j=0,1, \ldots, n$ seja $\gamma_{j} \cap D=\left\{p_{j}, p_{j+1}\right\}$ com $p_{0}=p_{n+1}$. Para cada $j=0,1, \ldots, n$ definimos um germe em $p_{j}$ de uma transformação de Poincaré $\pi_{j}:\left(D, p_{j}\right) \rightarrow\left(D, p_{j+1}\right)$ tal que a aplicação de primeiro retorno associada à órbita $\gamma$ é dada por

$$
\pi=\pi_{n} \circ \pi_{n-1} \circ \cdots \circ \pi_{0}
$$

$\operatorname{com} \pi\left(p_{0}\right)=p_{0}$.

A próxima figura apresenta a divisão da politrajetória fechada de acordo com a definição anterior para que seja associada a esta a aplicação de primeiro retorno.

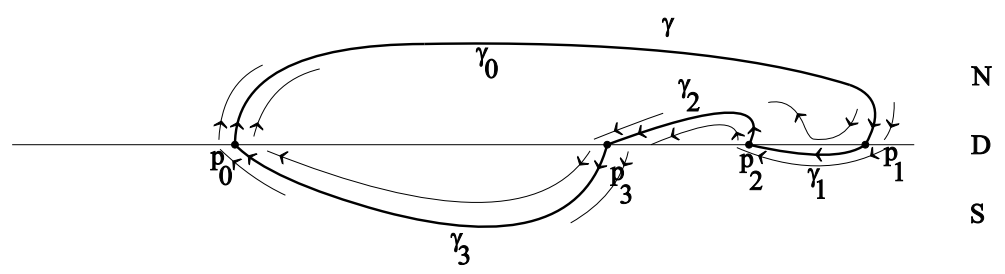

figura 15: politrajetória fechada do tipo I

Definição 12. Seja $\gamma$ uma politrajetória fechada, dizemos que $\gamma$ é elementar se satisfaz um dos seguintes requisitos

a) $\gamma$ é do tipo I e $\pi^{\prime}(p) \neq 1$ para algum ponto $p \in \gamma$

b) $\gamma$ é do tipo II 
c) $\gamma$ é do tipo III e todos os arcos de trajettória de $F_{Z}$ são de deslizamento ou todos os arcos são de escape.

\subsection{Regularização de campos vetoriais descontínuos}

Nesta seção vamos apresentar o método da regularização dos campos vetoriais descontínuos, conforme Sotomayor e Teixeira, e a definição de regularização que iremos considerar ao longo do texto. Vamos provar alguns resultados já conhecidos, sobre o ponto de vista da regularização de Sotomayor e Teixeira, para a nossa definição de regularização.

A regularização de um CVD é utilizada para estudar e interpretar a dinâmica desses campos através da teoria existente dos campos vetoriais contínuos. Em [ST] Sotomayor e Teixeira descrevem um método para regularizar os campos vetoriais descontínuos usando as funções de transição. Dizemos que $\psi: \mathbb{R} \rightarrow \mathbb{R}$ é uma função de transição se é de classe $C^{\infty}$, $\psi(x)=0$ se $x \leq-1, \psi(x)=1$ se $x \geq 1$ e $\psi^{\prime}(x)>0 \mathrm{em}(-1,1)$. Assim, a regularização de um CVD, conforme Sotomayor e Teixeira, é a família a um parâmetro de campos vetoriais contínuos

$$
Z_{\varepsilon}(q)=\left(1-\psi_{\varepsilon}(F(q))\right) Y(q)+\psi_{\varepsilon}(F(q)) X(q)
$$

onde $\varepsilon>0$ e $\psi_{\varepsilon}(x)=\psi(x / \varepsilon)$.

Porém, para os nossos propósitos, iremos definir a regularização usando a seguinte função auxiliar, no lugar da função de transição na definição acima,

$$
\varphi(x)=\frac{1}{2}+\frac{x}{2 \sqrt{x^{2}+1}} .
$$

Dado $\varepsilon>0$ usamos esta função para obter, de um campo vetorial descontínuo, uma família a um parâmetro real, $\varepsilon$, de campos vetoriais. $\mathrm{O}$ valor $\varepsilon$ é chamado de parâmetro de regularização.

Definição 13. Seja $Z=(X, Y) \in \Omega^{r}(M)$, onde $M$ é definida pela função $F: M \rightarrow \mathbb{R}$, sejam $\varepsilon>0$ e $\varphi$ a função definida em (2.6). Associada ao campo $Z$ definimos sua regularização como sendo a família de campos vetoriais indexada pelo parâmetro $\varepsilon, Z_{\varepsilon} \in \mathfrak{X}^{r}(M)$, dada por

$$
Z_{\varepsilon}(q)=\left(1-\varphi_{\varepsilon}(F(q))\right) Y(q)+\varphi_{\varepsilon}(F(q)) X(q)
$$

onde $\varphi_{\varepsilon}(x)=\varphi(x / \varepsilon)$.

A abreviatura FCR diz respeito a uma família de campos vetoriais regularizada, e CVR é a abreviatura de campo vetorial regularizado. O conjunto $D \times(-\varepsilon, \varepsilon)$ é chamado de região 
de regularização.

Para valores positivos de $\varepsilon$ temos a seguinte família de funções que será utilizada para descrever a regularização de um CVD

$$
\varphi_{\varepsilon}(x)=\varphi(x / \varepsilon)=\frac{1}{2}+\frac{x / \varepsilon}{2 \sqrt{(x / \varepsilon)^{2}+1}}=\frac{1}{2}+\frac{x}{2 \sqrt{x^{2}+\varepsilon^{2}}} .
$$

A próxima figura contém o gráfico de $\varphi_{\varepsilon}(x)$ para dois valores distintos de $\varepsilon$. No gráfico tracejado temos que $\varepsilon=0,2$ e no outro gráfico $\varepsilon=0,5$.

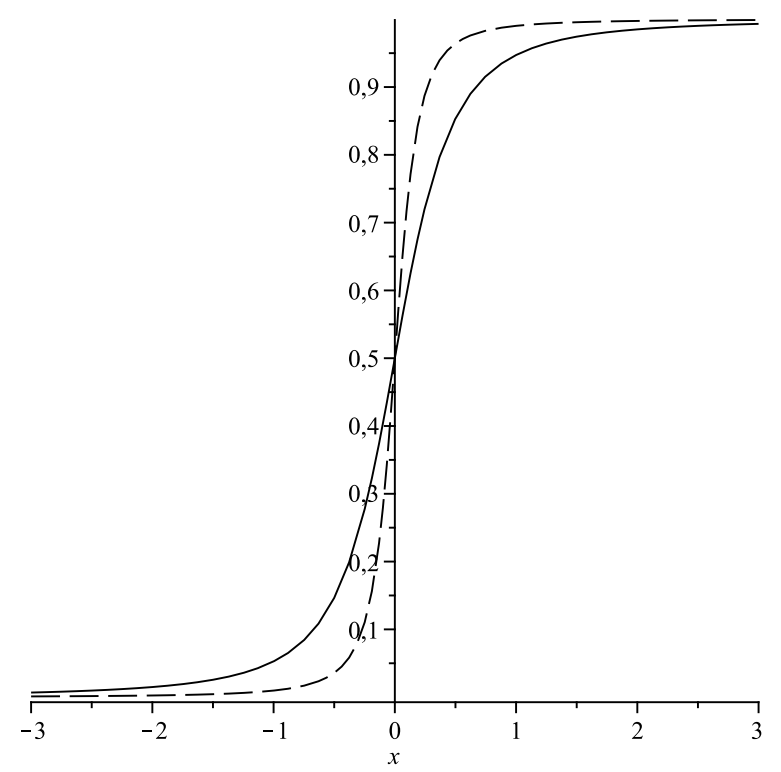

figura 17: função de transição

Assim, é fácil visualizar que a função (2.8) não é de fato uma função de transição, como definido por Sotomayor e Teixeira, visto que ela não se anula para valores menores que $-\varepsilon$ e também não vale 1 em valores maiores que $\varepsilon$. Além disso, a derivada da função (2.8) não tem suporte compacto. Os argumentos que usamos para considerar a função (2.8) no lugar de uma função de transição qualquer são que, em primeiro lugar a função (2.8) está tão próxima de uma função de transição qualquer quanto quisermos, bastando para isso tomarmos valores cada vez menores de $\varepsilon$. Além disso, tanto uma função de transição qualquer como a função (2.8) tendem à função de Heaviside (i. e., a função que vale 0 para valores negativos, 1 na semi-reta real positiva e $1 / 2$ na origem) quando $\varepsilon$ vai a zero. Esse é um argumento importante pois dado um CVD qualquer, o limite da família regularizada, quando $\varepsilon$ vai a zero, relacionada à função (2.8) e o limite da família regularizada, conforme 
Sotomayor e Teixeira, são os mesmos. Os fatos acima juntamente com a simplicidade da expressão de (2.8), donde a facilidade na manipulação algébrica das regularizações, são os argumentos para considerar essa função como sendo a função de transição nos cálculos das bifurcações dos campos vetoriais descontínuos.

Outro fato importante é a verificação de que as bifurcações independem da função de transição usada, mas isto não será analisado neste trabalho.

Obs.: Durante todo o texto, quando falarmos em função de transição estaremos nos referindo à família de funções de transição dada por (2.8).

Em $[\mathrm{M}]$ e $[\mathrm{SM}]$ há alguns resultados básicos que ocorrem na regularização de um CVD em vizinhanças de singularidades, trajetórias fechadas e de tangências quadráticas. Vamos verificar esses resultados para a função (2.8), sendo que as demonstrações são similares em vários pontos às provas apresentadas em $[\mathrm{M}]$ e $[\mathrm{SM}]$. Portanto, ao falarmos em campo vetorial regularizado estaremos nos referindo à família definida em (2.7). O primeiro resultado trata da regularização de um campo vetorial descontínuo que possua uma singularidade hiperbólica do campo de Filippov.

Proposição 3. Seja $p \in D$ uma singularidade hiperbólica de $F_{Z}$, onde $Z=(X, Y) \in \Omega^{r}(M)$. Então, existem uma vizinhança $V$ de $p$ em $M$ e $\varepsilon_{0}>0$ tais que para $0<\varepsilon<\varepsilon_{0}$, o campo vetorial regularizado $Z_{\varepsilon}$ tem uma única singularidade $p_{\varepsilon}$ em $V$, que é hiperbólica e do tipo sela ou nó, conforme $p$ o for para $F_{Z}$.

Demonstração. Para simplificar vamos considerar $p=(0,0)$ e que ao redor desse ponto as expressões dos campos $X$ e $Y$ são dadas por $X(x, y)=(a(x, y), b(x, y))$ e $Y(x, y)=$ $(c(x, y), d(x, y))$. As hipóteses sobre o ponto $p$ são que

1) $X F(p) Y F(p)=b(0,0) d(0,0)<0$

2) $\operatorname{det}(X, Y)(p)=a(0,0) d(0,0)-b(0,0) c(0,0)=0$

3) $d(\operatorname{det}(X, Y))_{D}(p)=a_{x}(p) d(p)+a(p) d_{x}(p)-b_{x}(p) c(p)-b(p) c_{x}(p) \neq 0$.

Vamos supor, sem perda de generalidade, que o ponto $p$ pertence ao conjunto de deslizamento, portanto temos que

$$
X F(p)=b(p)<0, \quad \text { e } \quad Y F(p)=d(p)>0 .
$$

O caso onde $p$ é um ponto de escape é tratado de modo análogo. 
O família regularizada de $Z$ é dada por

$$
Z_{\varepsilon}(x, y)=\left(\left(1-\varphi_{\varepsilon}\right) c+\varphi_{\varepsilon} a,\left(1-\varphi_{\varepsilon}\right) d+\varphi_{\varepsilon} b\right)
$$

e um ponto $q=(x, y)$ é singularidade dessa família se satisfizer

$$
\begin{aligned}
& \left(1-\varphi_{\varepsilon}(y)\right) c(x, y)+\varphi_{\varepsilon}(y) a(x, y)=0 \\
& \left(1-\varphi_{\varepsilon}(y)\right) d(x, y)+\varphi_{\varepsilon}(y) b(x, y)=0
\end{aligned}
$$

ou ainda, se valer que

$$
\frac{c(q)}{c(q)-a(q)}=\frac{d(q)}{d(q)-b(q)}
$$

implicando que $a(q) d(q)-b(q) c(q)=0$, ou seja, se $q$ é uma singularidade de $Z_{\varepsilon}$ então vale que $\operatorname{det}(X, Y)(q)=0$.

Pela hipótese 3) sobre $p$ temos que, pelo Teorema da Função Implícita, existe uma vizinhança $U_{1} \times U_{2} \subset \mathbb{R}^{2}$ de $p$ tal que $x=x(y)$ é diferenciável e

$$
\operatorname{det}(X, Y)(x(y), y)=a(x(y), y) d(x(y), y)-b(x(y), y) c(x(y), y)=0 \text {. }
$$

Nos pontos da curva $(x(y), y)$ vale que $\frac{c(q)}{c(q)-a(q)}=\frac{d(q)}{d(q)-b(q)}$.

Como estamos supondo que o ponto $p$ está no conjunto de deslizamento então $b(p)<0$ e $d(p)>0$, vamos considerar $\varepsilon_{0}>0$ tal que essas duas funções não mudem de sinal se $|x| \leq \varepsilon_{0}$ e $|y| \leq \varepsilon_{0}$, e ainda que $U_{2} \subset\left[-\varepsilon_{0}, \varepsilon_{0}\right]$. Assim, definimos

$$
V=\left\{(x, y) \in M:|x| \leq \varepsilon_{0},|y| \leq \varepsilon_{0}\right\}
$$

Seja $g: \mathbb{R} \rightarrow \mathbb{R}$ definida por

$$
g(y)=\frac{d(x(y), y)}{d(x(y), y)-b(x(y), y)} .
$$

Se $y \in\left[-\varepsilon_{0}, \varepsilon_{0}\right]$ então temos que $g(y) \in(0,1)$.

Sejam $k=\frac{d(p)}{d(p)-b(p)}$ e $m=$ mín $\{k, 1-k\}$. Se $(x, y) \in V$ e sendo $k \in(0,1)$ diminuimos $\varepsilon_{0}$ de modo que tenhamos

$$
\frac{d(x(y), y)}{d(x(y), y)-b(x(y), y)} \in\left(k-\frac{m}{2}, k+\frac{m}{2}\right) .
$$


Assim, a singularidade de $Z_{\varepsilon}$ em $V$ que estamos procurando será o ponto de interseção entre os gráficos de $g(y)$ e $\varphi_{\varepsilon}(y)$. Para verificar sua existência e unicidade vamos novamente usar o Teorema da Função Implícita.

A função $\varphi_{\varepsilon}: \mathbb{R} \rightarrow(0,1)$ é bijetora portanto, possui uma inversa. Queremos resolver o problema de achar um ponto $y(\varepsilon)$ que satisfaça $\varphi_{\varepsilon}(y(\varepsilon))=g(y(\varepsilon))$. Calculando a inversa da função $\varphi_{\varepsilon}$ e substituindo na igualdade acima obtemos que

$$
y(\varepsilon)-\frac{(2 g(y(\varepsilon))-1) \varepsilon}{2 \sqrt{g(y(\varepsilon))(1-g(y(\varepsilon)))}}=0 .
$$

Calculando a derivada em função de $y$ dessa igualdade e aplicando no ponto $\varepsilon=0$ obtemos que é diferente de zero. Portanto, pelo Teorema da Função Implícita temos a existência de um único ponto $p_{\varepsilon}=(x(\widetilde{y}(\varepsilon)), \widetilde{y}(\varepsilon))$ que satisfaz $y(0)=0$ e que é interseção entre os gráficos de $g(y)$ e de $\varphi_{\varepsilon}(y)$. Resta provar que essa singularidade é hiperbólica, e é uma sela se $p$ o for para $F_{Z}$ ou um nó caso $p$ seja um nó de $F_{Z}$.

A matriz Jacobiana de $Z_{\varepsilon}$ calculada em $p_{\varepsilon}$ é dada por

$$
D Z_{\varepsilon}\left(p_{\varepsilon}\right)=\left(\begin{array}{cc}
\left(1-\varphi_{\varepsilon}\right) c_{x}+\varphi_{\varepsilon} a_{x} & \left(\varphi_{\varepsilon}\right)^{\prime}(a-c)+\left(1-\varphi_{\varepsilon}\right) c_{y}+\varphi_{\varepsilon} a_{y} \\
\left(1-\varphi_{\varepsilon}\right) d_{x}+\varphi_{\varepsilon} b_{x} & \left(\varphi_{\varepsilon}\right)^{\prime}(b-d)+\left(1-\varphi_{\varepsilon}\right) d_{y}+\varphi_{\varepsilon} b_{y}
\end{array}\right)
$$

O polinômio característico, $\operatorname{det}\left(D Z_{\varepsilon}-\mu I d\right)=0$, tem a seguinte expressão

$$
\mu^{2}-\operatorname{tr}\left(D Z_{\varepsilon}\right) \mu+\operatorname{det}\left(D Z_{\varepsilon}\right)=0
$$

donde tiramos que os autovalores da matriz $D Z_{\varepsilon}$ aplicada na singularidade $p_{\varepsilon}$ são dados por

$$
\mu_{ \pm}=\frac{\operatorname{tr}\left(D Z_{\varepsilon}\right) \pm \sqrt{\operatorname{tr}^{2}\left(D Z_{\varepsilon}\right)-4 \operatorname{det}\left(D Z_{\varepsilon}\right)}}{2} .
$$

Denotando $\Delta=\operatorname{tr}^{2}\left(D Z_{\varepsilon}\right)-4 \operatorname{det}\left(D Z_{\varepsilon}\right)$ (vamos supor que $\Delta \geq 0$, o caso negativo não será analisado) e $B=-\operatorname{tr}\left(D Z_{\varepsilon}\right)$ temos que $p_{\varepsilon}$ será uma sela hiperbólica se

$$
\begin{array}{lll}
-B+\sqrt{\Delta}>0 & \Rightarrow & \sqrt{\Delta}>B \\
-B-\sqrt{\Delta}<0 & \Rightarrow & \sqrt{\Delta}<B
\end{array}
$$

donde concluímos que $\Delta>B^{2}$. 
E será um nó hiperbólico quando tivermos que

$$
(-B+\sqrt{\Delta})(-B-\sqrt{\Delta})>0
$$

implicando que $0 \leq \Delta<B^{2}$.

Como $\Delta-B^{2}=-4 \operatorname{det}\left(D Z_{\varepsilon}\right)$, basta analisar o sinal do determinante Jacobiano do campo regularizado calculado em $p_{\varepsilon}=\left(x_{\varepsilon}, y_{\varepsilon}\right)$. Manipulando algebricamente a expressão do determinante Jacobiano obtemos

$$
\begin{aligned}
\operatorname{det}\left(D Z_{\varepsilon}\right)\left(p_{\varepsilon}\right) & =\left(\varphi_{\varepsilon}\right)^{\prime}\left(b c_{x}-a d_{x}-a_{x} d+b_{x} c\right)+L \\
& =-\left(\varphi_{\varepsilon}\right)^{\prime}\left(p_{\varepsilon}\right) d(\operatorname{det}(X, Y))_{D}\left(p_{\varepsilon}\right)+L\left(p_{\varepsilon}\right)
\end{aligned}
$$

onde

$$
\begin{aligned}
& L\left(p_{\varepsilon}\right)=\left(c_{x}\left(p_{\varepsilon}\right) d_{y}\left(p_{\varepsilon}\right)-c_{y}\left(p_{\varepsilon}\right) d_{x}\left(p_{\varepsilon}\right)\right)+\varphi_{\varepsilon}\left(y_{\varepsilon}\right)\left[-2 c_{x}\left(p_{\varepsilon}\right) d_{y}\left(p_{\varepsilon}\right)+2 c_{y}\left(p_{\varepsilon}\right) d_{x}\left(p_{\varepsilon}\right)+b_{y}\left(p_{\varepsilon}\right) c_{x}\left(p_{\varepsilon}\right)\right. \\
& \left.\quad+a_{x}\left(p_{\varepsilon}\right) d_{y}\left(p_{\varepsilon}\right)-a_{y}\left(p_{\varepsilon}\right) d_{x}\left(p_{\varepsilon}\right)-b_{x}\left(p_{\varepsilon}\right) c_{y}\left(p_{\varepsilon}\right)\right]+\left(\varphi_{\varepsilon}\left(y_{\varepsilon}\right)\right)^{2}\left[c_{x}\left(p_{\varepsilon}\right) d_{y}\left(p_{\varepsilon}\right)-c_{y}\left(p_{\varepsilon}\right) d_{x}\left(p_{\varepsilon}\right)\right. \\
& \left.\quad-b_{y}\left(p_{\varepsilon}\right) c_{x}\left(p_{\varepsilon}\right)-a_{x}\left(p_{\varepsilon}\right) d_{y}\left(p_{\varepsilon}\right)+a_{y}\left(p_{\varepsilon}\right) d_{x}\left(p_{\varepsilon}\right)+b_{x}\left(p_{\varepsilon}\right) c_{y}\left(p_{\varepsilon}\right)+a_{x}\left(p_{\varepsilon}\right) b_{y}\left(p_{\varepsilon}\right)-a_{y}\left(p_{\varepsilon}\right) b_{x}\left(p_{\varepsilon}\right)\right]
\end{aligned}
$$

é uma função limitada.

A derivada de $\varphi_{\varepsilon}$ calculada em $p_{\varepsilon}$ é dada por

$$
\varphi_{\varepsilon}^{\prime}\left(p_{\varepsilon}\right)=\frac{\varepsilon^{2}}{2\left(y_{\varepsilon}^{2}+\varepsilon^{2}\right)^{3 / 2}}=\frac{\varepsilon^{2}}{2\left(\left(y_{\varepsilon} / \varepsilon\right)^{2}+1\right)^{3 / 2} \varepsilon^{3}}=\frac{1}{2\left(\left(y_{\varepsilon} / \varepsilon\right)^{2}+1\right)^{3 / 2} \varepsilon} .
$$

Como $y_{\varepsilon}$ é diferenciável, de (2.9) e da definição da função $g$ obtemos que $\frac{y_{\varepsilon}}{\varepsilon}$ é limitada, implicando que

$$
\lim _{\varepsilon \rightarrow 0^{+}} \varphi_{\varepsilon}^{\prime}\left(p_{\varepsilon}\right)=+\infty .
$$

Assim, concluímos que o sinal de $\operatorname{det}\left(D Z_{\varepsilon}\right)\left(p_{\varepsilon}\right)$ é o mesmo de $-d(\operatorname{det}(X, Y))_{D}\left(p_{\varepsilon}\right)$, ou seja, $p_{\varepsilon}$ é uma sela hiperbólica de $Z_{\varepsilon}$ se $p$ o for para $F_{Z}$, ou será um nó hiperbólico de $Z_{\varepsilon}$ se $p$ o for para $F_{Z}$.

No próximo resultado apresentamos a regularização de um ponto $D$-regular de $Z$.

Proposição 4. Seja $p \in D$ um ponto D-regular de $Z=(X, Y) \in \Omega^{r}(M), r \geq 1$. Então, existem uma vizinhança $V$ de $p$ em $M$ e $\varepsilon_{0}>0$ tais que para $0<\varepsilon<\varepsilon_{0}$, o campo vetorial regularizado $Z_{\varepsilon}$ não tem singularidades em $V$. 
Demonstração. Seja $p=(0,0)$ um ponto $D$-regular do campo vetorial descontínuo $Z=$ $(X, Y)$ dado por $X(x, y)=(a(x, y), b(x, y))$ e $Y(x, y)=(c(x, y), d(x, y))$. Com relação à posição do ponto $p$ considerando os conjuntos de costura, deslizamento e escape temos dois casos a considerar:

I) Se $p$ é um ponto de costura, ou seja, $X F(p) Y F(p)=b(p) d(p)>0$. Vamos supor, sem perda de generalidade, que $b(p)>0$ e $d(p)>0$. Seja $V=\{(x, y) \in M: b(x, y)>$ $0, d(x, y)>0\}$ uma vizinhança de $p$. Vamos verificar que em $V$ o campo regularizado não possui singularidades. A expressão do campo regularizado é

$$
Z_{\varepsilon}(x, y)=\left(\left(1-\varphi_{\varepsilon}(y)\right) c(x, y)+\varphi_{\varepsilon}(y) a(x, y),\left(1-\varphi_{\varepsilon}(y)\right) d(x, y)+\varphi_{\varepsilon}(y) b(x, y)\right)
$$

Como em $V$ as funções $b$ e $d$ são estritamente positivas, e considerando que a função $\varphi_{\varepsilon}$ nunca se anula, temos que a segunda componente de $Z_{\varepsilon}$ nunca se anula, para qualquer valor de $\varepsilon>0$, ou seja, não existem singularidades do campo regularizado em $V$.

II) Se $p$ é um ponto $D$-regular de $F_{Z}$ onde $X F(p) Y F(p)=b(p) d(p)<0 \operatorname{edet}(X, Y)(p)=$ $a(p) d(p)-b(p) c(p) \neq 0$. Vamos supor, sem perda de generalidade, que $p$ é um ponto de escape, ou seja, $b(p)>0$ e $d(p)<0$. Seja $V=\{(x, y) \in M: a(x, y) d(x, y)-b(x, y) c(x, y) \neq 0\}$ uma vizinhança de $p$. A expressão do campo regularizado é dada por (2.10), e um ponto $(x, y)$ será uma singularidade desse campo se satisfizer

$$
\frac{c(x, y)}{c(x, y)-a(x, y)}=\frac{d(x, y)}{d(x, y)-b(x, y)}
$$

implicando que $a(x, y) d(x, y)-b(x, y) c(x, y)=\operatorname{det}(X, Y)(x, y)=0$. Mas, qualquer ponto $(x, y) \in V$ não satisfaz essa iguladade, para qualquer valor de $\varepsilon$, donde o campo regularizado não tem singularidades em $V$.

O seguinte corolário é consequência da proposição anterior.

Corolário 1. Sejam $Z=(X, Y) \in \Omega^{r}(M)$ e $T \subset D$ um conjunto compacto de pontos $D$ regulares. Então, existem uma vizinhança $V$ de $T$ em $M$ e $\varepsilon_{0}>0$ tais que para $0<\varepsilon<\varepsilon_{0}$, o campo vetorial regularizado $Z_{\varepsilon}$ não tem singularidades em $V$.

O próximo resultado que apresentaremos trata da regularização de uma $D$-dobra de $Z$.

Proposição 5. Seja $p \in D$ uma $D$-dobra de $Z=(X, Y) \in \Omega^{r}(M), r \geq 1$. Então, existem uma vizinhança $V$ de p em $M$ e $\varepsilon_{0}>0$ tais que para $0<\varepsilon<\varepsilon_{0}$, o campo vetorial regularizado $Z_{\varepsilon}$ não possui singularidades em $V$. 
Demonstração. Vamos considerar $p=(0,0)$ e que o campo vetorial descontínuo é dado por $Z=(X, Y)$ onde $X(x, y)=(a(x, y), b(x, y))$ e $Y(x, y)=(c(x, y), d(x, y))$. Vamos supor, sem perda de generalidade, que as hipóteses sobre $p$ são dadas por

1) $X F(p)=b(p)=0$

2) $X^{2} F(p)=a(p) b_{x}(p)+b(p) b_{y}(p)=a(p) b_{x}(p)>0$, implicando que $a(p) \neq 0$

3) $Y F(p)=d(p)>0$.

Os outros casos são análogos.

A expressão do campo regularizado $Z_{\varepsilon}$ é dada por (2.10). Seja $\varepsilon>0$ de modo que as funções $a(x, y)$ e $d(x, y)$ não se anulem quando $|x| \leq \varepsilon_{0}$ e $|y| \leq \varepsilon_{0}$. Definimos $V=\{(x, y) \in$ $\left.M:|x| \leq \varepsilon_{0},|y| \leq \varepsilon_{0}\right\}$ a vizinhança de $p$. Notamos que em $V$ as funções $a$ e $d$ não se anulam, e a função $\varphi_{\varepsilon}$ nunca se anula, $\forall \varepsilon>0$. Assim, o campo regularizado não possui nenhuma singularidade em $V$.

As politrajetórias fechadas são divididas nos tipos I, II e III, conforme a definição 10 da seção anterior. Por se tratar da mais complexa dessas três, vamos apresentar a regularização de um CVD que possua uma politrajetória fechada elementar do tipo III. A regularização das politrajetórias fechadas do tipo I e II, cuja prova será omitida, são obtidas similarmente ao apresentado em $[\mathrm{M}]$ e $[\mathrm{SM}]$, bastando para isso fazer os ajustes necessários na mudança da função de transição para a função (2.8).

Proposição 6. Seja $\gamma$ uma politrajetória fechada elementar do tipo III de $Z=(X, Y) \in$ $\Omega^{r}(M), r \geq 1$. Então, existem uma vizinhança $V$ de $\gamma$ em $M e \varepsilon_{0}>0$ tais que para $0<\varepsilon<\varepsilon_{0}$, o campo vetorial regularizado $Z_{\varepsilon}$ contém uma única órbita periódica hiperbólica atratora em $V$.

Demonstração. Seja $\gamma$ uma politrajetória fechada elementar do tipo III de $Z$. Vamos supor, sem perda de generalidade, que $\gamma$ possui um único arco de trajetória do campo $X$, e um único arco de trajetória de $F_{Z}$, contido no conjunto de deslizamento. Sejam $\Sigma_{i}, i=1,2,3$, 4, seções transversais à $\gamma$, disposta como na figura seguinte.

Deste modo, as órbitas que saem de $\Sigma_{1} \cap S$ cruzam o conjunto de deslizamento e as órbitas próximas de $\gamma$ que cruzam $\Sigma_{4}$ vão até $\Sigma_{1}$ pelo conjunto de deslizamento. Sejam $r_{i} \in \Sigma_{i} \cap \gamma$, $i=1,2,3,4$.

Vamos considerar que a seção transversal $\Sigma_{3}$ está munida de uma orientação, cujos valores maiores apontam para o interior da região compacta limitada por $\gamma$ e $D$. Sejam $q_{0}, q_{1} \in \Sigma_{3}$ 


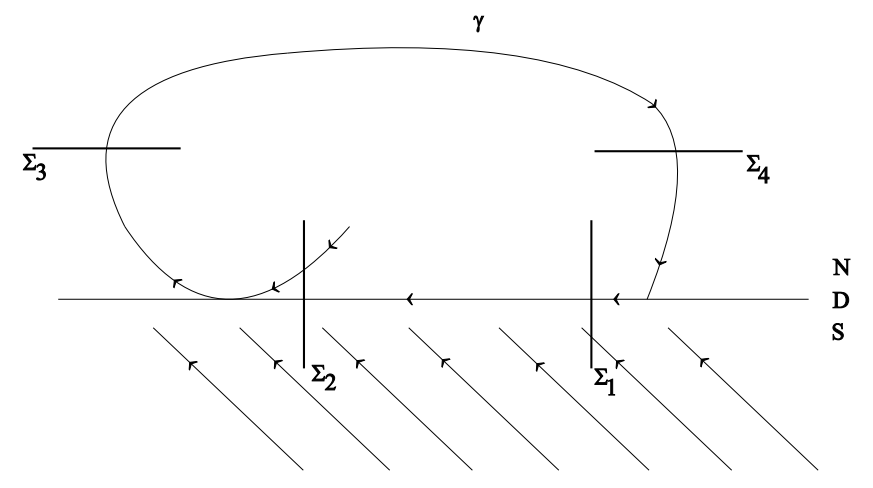

figura 18: politrajetória $\gamma$

tais que $q_{0}<r_{3}<q_{1}$. Sejam $q_{3}=\left(m_{3}, n_{3}\right)$ o ponto da seção $\Sigma_{2}$ cuja órbita de $X$ passa por $q_{1}$, e $q_{4}=\left(m_{4}, n_{4}\right)$ o ponto da órbita de $X$ que passa por $q_{1}$ e cruza $\Sigma_{1}$. Seja $n_{5}<0$ de modo que as órbitas de $Y$ passando por $(x, y)$, com $0>y>n_{5}$, em $\Sigma_{1}$ entram no arco deslizante. Seja $V$ o anel contendo $\gamma$ que tem como bordo interno a órbita de $X$ que passa por $q_{1}$ de $\Sigma_{2}$ a $\Sigma_{1}$, pelo segmento de reta contido em $\Sigma_{1}$ que está entre $y=n_{4}$ e $y=n_{3}$, e pelo segmento de reta entre $\Sigma_{1}$ e $\Sigma_{2}$ dado por $y=n_{6}$ onde $n_{6}=\operatorname{mín}\left\{n_{3}, n_{4}\right\}$. O bordo externo de $V$ é formado pela trajetória de $X$ que passa por $q_{0}$ até $D$ unido com o conjunto $D$ até $\Sigma_{1}$, o segmento de reta em $\Sigma_{1}$ que vai de $y=0$ até $y=n_{5}$, o segmento de reta que vai de $\Sigma_{1}$ até $\Sigma_{2}$ na altura $y=n_{5}$. Se $\varepsilon_{0}$ for considerado de maneira que $\varepsilon_{0} \leq n_{6}$ e $-\varepsilon_{0} \geq n_{5}$ e as seções transversais $\Sigma_{3}$ e $\Sigma_{4}$ estão fora da região de regularização, então para $0<\varepsilon<\varepsilon_{0}$, as órbitas do campo regularizado $Z_{\varepsilon}$ estão entrando no anel $V$.

Como em $V$ não existem singularidades, pelo Teorema de Poincaré-Bendixson, o campo $Z_{\varepsilon}$ possui pelo menos uma órbita periódica, $\gamma_{\varepsilon}$, em $V$. Como as seções transversais de $\gamma$ desempenham o mesmo papel para $\gamma_{\varepsilon}$, resta calcular a derivada da aplicação de primeiro retorno $\pi_{\varepsilon}$ da órbita periódica $\gamma_{\varepsilon}$ definida em $\Sigma_{1}$. Para isso, definimos a aplicação de primeiro retorno por

$$
\pi_{\varepsilon}=\pi_{4, \varepsilon} \circ \pi_{3, \varepsilon} \circ \pi_{2, \varepsilon} \circ \pi_{1, \varepsilon}
$$

onde $\pi_{i, \varepsilon}$ está definida entre $\Sigma_{i}$ e $\Sigma_{i+1}, i=1,2,3,4$, identificando $\Sigma_{5}=\Sigma_{1}$.

Sejam $p_{i}=\left(x_{i}, y_{i}\right) \in \Sigma_{i} \cap \gamma_{\varepsilon}$, e $\theta_{i}$ os ângulos formados entre as seções transversais e $\gamma_{\varepsilon}$, $i=1,2,3$, 4. Agora vamos calcular a derivada da aplicação de primeiro retorno entre as seções transversais.

1. Cálculo de $\pi_{1, \varepsilon}^{\prime}$

Vamos considerar que os campos vetoriais são dados por $X(x, y)=(-1,-1)$ e $Y(x, y)=$ 
$(-1,1)$. Assim, o campo regularizado é dado por

$$
Z_{\varepsilon}(x, y)=\left(-1,1-2 \varphi_{\varepsilon}(y)\right)=\left(-1,-\frac{y}{\sqrt{y^{2}+\varepsilon^{2}}}\right)
$$

Como $x_{1}$ e $x_{2}$ são as abscissas das seções transversais $\Sigma_{1}$ e $\Sigma_{2}$, respectivamente, podemos reduzir o campo vetorial regularizado a $\frac{d y}{d x}=\frac{1-2 \varphi_{\varepsilon}}{-1}$ que implica em

$$
\int_{y_{1}}^{y_{2}} \frac{1}{1-2 \varphi_{\varepsilon}(y)} d y=\int_{x_{1}}^{x_{2}}-1 d x=x_{1}-x_{2}
$$

Seja

$$
\psi\left(y_{1}\right)=\int_{y_{1}}^{y_{2}} \frac{1}{1-2 \varphi_{\varepsilon}(y)} d y=x_{1}-x_{2}=H\left(y_{2}\right)-H\left(y_{1}\right)
$$

Portanto,

$$
\frac{\partial \psi\left(y_{1}\right)}{\partial y_{1}}=H^{\prime}\left(y_{2}\right) \frac{d y_{2}}{d y_{1}}-H^{\prime}\left(y_{1}\right)=\frac{1}{1-2 \varphi_{\varepsilon}\left(y_{2}\right)} \frac{d y_{2}}{d y_{1}}-\frac{1}{1-2 \varphi_{\varepsilon}\left(y_{1}\right)}=0
$$

que implica

$$
0<\frac{d y_{2}}{d y_{1}}=\frac{1-2 \varphi_{\varepsilon}\left(y_{2}\right)}{1-2 \varphi_{\varepsilon}\left(y_{1}\right)}<1
$$

Com a nossa função de transição temos que $\varphi_{\varepsilon}(0)=1 / 2$ para qualquer valor de $\varepsilon>0$. Além disso, quando $\varepsilon$ vai a zero temos que $y_{2}$ tende a $\varphi^{-1}(1 / 2)=0$.

Finalmente, temos que a derivada da aplicação de Poincaré de $\gamma_{\varepsilon}$ entre $\Sigma_{1}$ e $\Sigma_{2}$ vale

$$
\begin{aligned}
\pi_{1, \varepsilon}^{\prime}\left(p_{1}\right) & =\frac{\cos \theta_{1}\left|Z_{\varepsilon}\left(p_{1}\right)\right|}{\cos \theta_{2}\left|Z_{\varepsilon}\left(p_{2}\right)\right|} \exp \left(\int_{t_{1}}^{t_{2}} \operatorname{div} Z_{\varepsilon}\left(\gamma_{\varepsilon}(t)\right) d t\right) \\
& =\frac{\cos \theta_{1}\left|Z_{\varepsilon}\left(p_{1}\right)\right|\left|1-2 \varphi_{\varepsilon}\left(y_{2}\right)\right|}{\cos \theta_{2}\left|Z_{\varepsilon}\left(p_{2}\right)\right|\left|1-2 \varphi_{\varepsilon}\left(y_{1}\right)\right|}
\end{aligned}
$$

2. Cálculo de $\pi_{2, \varepsilon}^{\prime}$

Nesse caso consideramos que $X(x, y)=(-1,-x)$ e $Y(x, y)=(-1,1)$ donde o ponto de dobra é $p=(0,0)$. O campo regularizado é $Z_{\varepsilon}=\left(-1,1-\varphi_{\varepsilon}-x \varphi_{\varepsilon}\right)$ e procedendo como no caso anterior, temos que $\frac{d t}{d y}=\frac{1}{1-\varphi_{\varepsilon}-x \varphi_{\varepsilon}}$ implicando que a derivada da aplicação de 
Poincaré de $\gamma_{\varepsilon}$ entre $\Sigma_{2}$ e $\Sigma_{3}$ é

$$
\begin{aligned}
\pi_{2, \varepsilon}^{\prime}\left(p_{2}\right) & =\frac{\cos \theta_{2}\left|Z_{\varepsilon}\left(p_{2}\right)\right|}{\cos \theta_{3}\left|Z_{\varepsilon}\left(p_{3}\right)\right|} \exp \left(\int_{t_{2}}^{t_{3}} \operatorname{div} Z_{\varepsilon}\left(\gamma_{\varepsilon}(t)\right) d t\right) \\
& =\frac{\cos \theta_{2}\left|Z_{\varepsilon}\left(p_{2}\right)\right|\left|1-\varphi_{\varepsilon}\left(y_{3}\right)\left(1+x_{3}\right)\right|}{\cos \theta_{3}\left|Z_{\varepsilon}\left(p_{3}\right)\right|\left|1-\varphi_{\varepsilon}\left(y_{2}\right)\left(1+x_{2}\right)\right|}
\end{aligned}
$$

3. Cálculo de $\pi_{3, \varepsilon}^{\prime}$

Nesse caso para $\varepsilon$ suficientemente pequeno a órbita $\gamma_{\varepsilon}$ está fora da região de regularização, portanto aproximamos essa órbita por pontos de $X$. Assim, a derivada da aplicação de Poincaré de $\gamma_{\varepsilon}$ entre $\Sigma_{3}$ e $\Sigma_{4}$ é

$$
\pi_{3, \varepsilon}^{\prime}\left(p_{3}\right)=\frac{\cos \theta_{3}\left|Z_{\varepsilon}\left(p_{3}\right)\right|}{\cos \theta_{4}\left|Z_{\varepsilon}\left(p_{4}\right)\right|} K
$$

onde $K$ é uma constante real.

4. Cálculo de $\pi_{4, \varepsilon}^{\prime}$

Como simplificação vamos considerar as mesmas expressões de $Z$ daquelas expressas entre as seções $\Sigma_{1}$ e $\Sigma_{2}$. Portanto,

$$
\pi_{4, \varepsilon}^{\prime}\left(p_{4}\right)=\frac{\cos \theta_{4}\left|Z_{\varepsilon}\left(p_{4}\right)\right|\left|1-2 \varphi_{\varepsilon}\left(y_{1}\right)\right|}{\cos \theta_{1}\left|Z_{\varepsilon}\left(p_{1}\right)\right|\left|1-2 \varphi_{\varepsilon}\left(y_{4}\right)\right|}
$$

Compondo essas derivadas temos que

$$
\pi_{\varepsilon}^{\prime}\left(p_{1}\right)=\pi_{4, \varepsilon}^{\prime}\left(p_{4}\right) \pi_{3, \varepsilon}^{\prime}\left(p_{3}\right) \pi_{2, \varepsilon}^{\prime}\left(p_{2}\right) \pi_{1, \varepsilon}^{\prime}\left(p_{1}\right)=K \frac{\left|1-\varphi_{\varepsilon}\left(y_{3}\right)\left(1+x_{3}\right)\right|\left|1-2 \varphi_{\varepsilon}\left(y_{2}\right)\right|}{\left|1-2 \varphi_{\varepsilon}\left(y_{4}\right)\right|\left|1-\varphi_{\varepsilon}\left(y_{2}\right)\left(1+x_{2}\right)\right|}
$$

Agora, como $y_{2}$ vai a zero quando $\varepsilon$ tende a zero, e sendo que $\varphi(0)=1 / 2$ para qualquer valor de $\varepsilon$ segue que $\lim _{\varepsilon \rightarrow 0} \pi_{\varepsilon}^{\prime}\left(p_{1}\right)=0$. Implicando que para valores suficientemente pequenos de $\varepsilon$ podemos garantir que $\pi_{\varepsilon}^{\prime}\left(p_{1}\right)<1$, implicando que $\gamma_{\varepsilon}$ é uma órbita periódica hiperbólica atratora, e única. Os cálculos acima foram feitos para coordenadas simplificadas, mas a conclusão é verdadeira para o caso geral, onde as contas são mais complexas.

Para finalizar esta seção vamos apresentar um resultado que relaciona as variedades de um nó atrator de Filippov com as variedades do respectivo nó do campo regularizado.

Proposição 7. Seja $Z=(X, Y)$ um campo vetorial descontínuo que possua em $p=(0,0)$ uma singularidade hiperbólica de Filippov que é um nó atrator. Então o campo regularizado $Z_{\varepsilon}$ possui um nó hiperbólico atrator cujas variedades tendem às respectivas variedades de $p$. 
Demonstração. Com uma mudança de variáveis apropriada podemos supor que, em uma vizinhança de $p=(0,0)$, os campos $X$ e $Y$ têm a seguinte expressão $X(x, y)=(P(x, y), Q(x, y))$ e $Y(x, y)=(0,1)$. As hipóteses iniciais sobre o ponto $p$ são que $P(0,0)=0, \frac{\partial P}{\partial x}(0,0)<0$ e $Q(0,0)<0$. Deste modo, temos que o ponto $p$ é um nó atrator em um conjunto de deslizamento. A figura que retrata este caso é a seguinte.

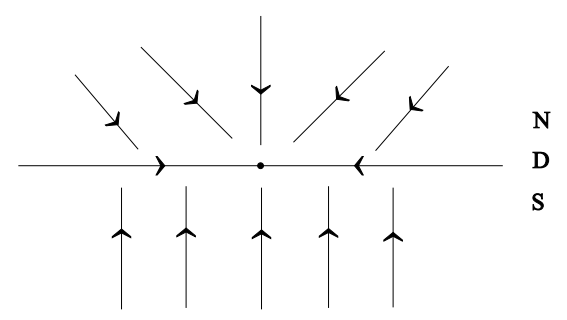

figura 19: nó atrator de Filippov

Para provarmos esta proposição basta considerarmos um conjunto que contém o ponto $p$ e verificarmos que tanto para $p$ como para o nó do campo regularizado, o comportamento dos respectivos campos vetoriais, no bordo dessa vizinhança, não se altera para valores pequenos e positivos do parâmetro de regularização.

O conjunto que iremos considerar será denotado por $V$ e é simétrico com relação ao eixo vertical, a parte que está no semiplano de abscissa positiva é formada por três segmentos de retas, o primeiro está contido na reta $y=k x, k>0$, o segundo na reta $y=-k x$ e o terceiro na reta $x=m$, para $m>0$, de modo que formem no primeiro e quarto quadrantes um triângulo isósceles. A próxima figura apresenta esta vizinhança.

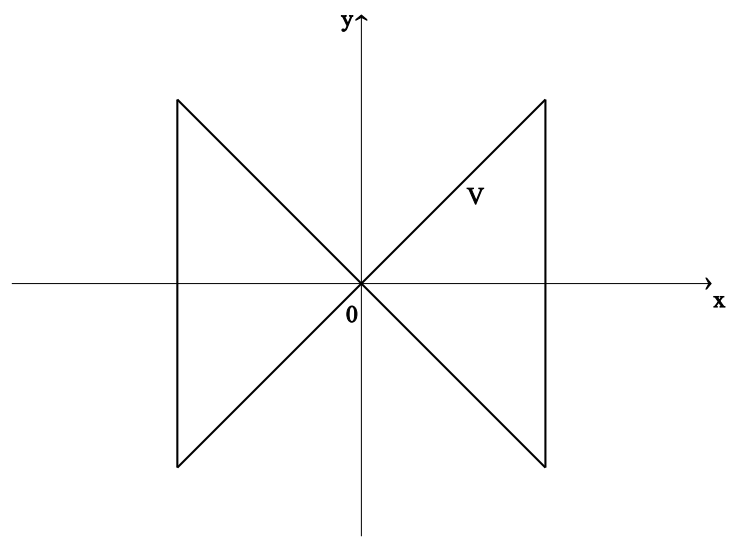

figura 20: conjunto $\mathrm{V}$

Uma vez que temos este conjunto sabemos como se comporta o campo $Z$ aplicado no bordo de $V$. Levando em consideração o conjunto de deslizamento temos que as trajetórias 
de $Z$ estão quase todas entrando em $V$. A próxima figura apresenta este comportamento.

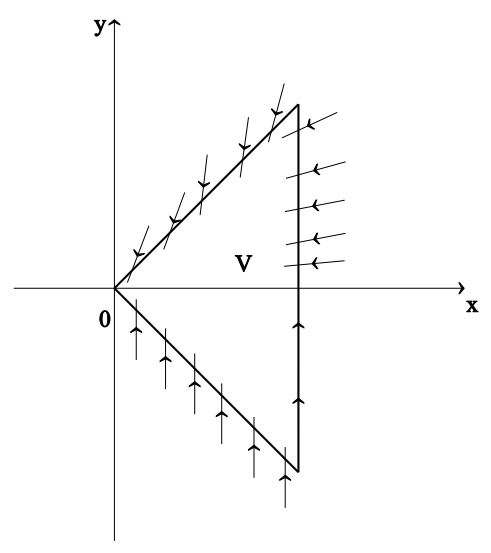

figura 21: comportamento de $Z$ em $V$

Agora vamos regularizar $Z$ e verificar o comportamento de $Z_{\varepsilon}$ no bordo de $V$.

O campo regularizado é dado por

$$
\begin{aligned}
& x^{\prime}=\left(\frac{1}{2}+\frac{y}{2 \sqrt{y^{2}+\varepsilon^{2}}}\right) P(x, y) \\
& y^{\prime}=\frac{1}{2}-\frac{y}{2 \sqrt{y^{2}+\varepsilon^{2}}}+\left(\frac{1}{2}+\frac{y}{2 \sqrt{y^{2}+\varepsilon^{2}}}\right) Q(x, y) .
\end{aligned}
$$

Vamos efetuar a seguinte mudança de variáveis em (2.12)

$$
\left\{\begin{array}{l}
x=w \\
y=z w
\end{array}\right.
$$

obtemos então

$$
\begin{aligned}
& w^{\prime}=\left(\frac{1}{2}+\frac{z w}{2 \sqrt{z^{2} w^{2}+\varepsilon^{2}}}\right) P(w, z w) \\
& z^{\prime}=\frac{1}{2}-\frac{z w}{2 \sqrt{z^{2} w^{2}+\varepsilon^{2}}}+\left(\frac{1}{2}+\frac{z w}{2 \sqrt{z^{2} w^{2}+\varepsilon^{2}}}\right) Q(w, z w) .
\end{aligned}
$$

Assim, com essa mudança de variáveis os segmentos de reta que formam os lados do triângulo da direita em $V$, são levados em segmentos de reta cuja altura é dada pela inclinação da reta em $V$, ou seja, $k$. Vamos verificar o comportamento de $Z_{\varepsilon}$, dado por (2.14), quando $z$ for igual a $k,-k$ e quando $w=m$. Em suma, esperamos que $z^{\prime}(w, k)<0, z^{\prime}(w,-k)>0$ e $w^{\prime}(m, z)<0$ 
pois, assim, o campo estaria entrando em $V$, tendo portanto o mesmo comportamento que o campo descontínuo nesse conjunto.

Substituindo $z=k$ em $z^{\prime}$ obtemos

$$
z^{\prime}(w, k)=\frac{1+Q(w, k)-k P(w, k)}{2 w}-\frac{k w}{2 w \sqrt{k^{2} w^{2}+\varepsilon^{2}}}(1-Q(w, k)+k P(w, k))
$$

Como estamos interessados em valores positivos e muito pequenos do parâmetro de regularização, vamos tomar o limite de (2.15) quando $\varepsilon$ vai a zero por valores positivos. Levando em consideração que $k>0$ e $w>0$ obtemos então

$$
\lim _{\varepsilon \rightarrow 0^{+}} z^{\prime}(w, k)=\frac{Q(w, k)-k P(w, k)}{w} .
$$

Resta agora verificarmos o sinal deste limite, para isso, vamos reescrever o resultado do limite acima nas variáveis originais

$$
\frac{Q\left(x, k x^{2}\right)-k P\left(x, k x^{2}\right)}{x} .
$$

Em uma vizinhança de $p$ escrevemos $P$ como

$$
P(x, y)=\frac{\partial P}{\partial x}(p) x+\frac{\partial P}{\partial y}(p) y+O(x, y)
$$

onde $O(x, y)$ é o resto com termos de ordem maior ou igual a 2. Da hipótese inicial sobre $p$ temos que $\frac{\partial P}{\partial x}(p)<0$. Portanto, este é o primeiro coeficiente, do polinômio que exprime $P$, que não se anula. Vamos utilizar essa aproximação para $P$, ou seja, vamos considerar que $P(x, y) \approx \frac{\partial P}{\partial x}(p) x$. Subsituindo essa aproximação em (2.17) obtemos

$$
\frac{Q\left(x, k x^{2}\right)}{x}-k \frac{\partial P}{\partial x}(p)
$$

Agora, como estamos supondo que $Q(0,0)<0$ e $-k \frac{\partial P}{\partial x}(p)>0$ então temos que (2.18) tende a $-\infty$ quando $x \rightarrow 0^{+}$implicando, portanto, que para valores positivos e pequenos de $x$ temos que $z^{\prime}(w, k)$ tende a um valor negativo quando o parâmetro de regularização é positivo e suficientemente pequeno.

Agora vamos verificar $z^{\prime}(w, k)$, para $k<0$. Substituindo $z=k$ em $z^{\prime}$ obtemos a expressão dada por (2.15). Tomando o limite quando $\varepsilon$ vai a 0 por valores positivos e considerando $w>0$ e $k<0$ otemos então que $z^{\prime}(w, k) \rightarrow \frac{1}{w}>0$.

Para finalizar vamos verificar $w^{\prime}(m, z)$ onde $m>0$. Substituindo $w=m$ na expressão de 
$w^{\prime}$ obtemos

$$
w^{\prime}(m, z)=\left(\frac{1}{2}+\frac{z m}{2 \sqrt{z^{2} m^{2}+\varepsilon^{2}}}\right) P(m, z m)
$$

o limite quando $\varepsilon$ vai a zero por valores positivos é dado por

$$
\frac{P(m, z m)}{2}+\frac{z m P(m, z m)}{2|z m|} .
$$

Agora, levando em consideração o sinal de z obtemos

$$
\begin{cases}P(m, z m), & z>0 \\ 0, & z<0\end{cases}
$$

Como estamos considerando a primeira aproximação de $P$, ou seja, $P(x, y)=\frac{\partial P}{\partial x}(p) x$ e como $\frac{\partial P}{\partial x}(p)<0$ e $x>0$ temos então que $P(x, y)<0$ implicando que quando $y>0$ o campo $Z_{\varepsilon}$ aponta para dentro de $V$. Para valores negativos de $z$, considerando as contas já efetuadas de $z^{\prime}(w, k)$ com $k<0$, temos que o campo regularizado aponta para o conjunto de descontinuidade.

O caso onde $Z$ tem um nó repulsor de Filippov é tratado de modo análogo.

\subsection{Campos vetoriais lineares e constantes}

Nos capítulos 3, 4, 5 e 6 estudaremos famílias de campos vetoriais descontínuos a um parâmetro real, $\lambda$, que possuam uma singularidade de $X$ em $N$, para valores negativos e pequenos do parâmetro, que colide com o conjunto de descontinuidade na origem, quando o parâmetro se anula. Vamos considerar a família $Y_{\lambda}$ em $S$ como sendo constante. Estaremos supondo que a função que define o conjunto $M$ é $F: M \rightarrow \mathbb{R}$ dada por $F(x, y)=y$. Assim, $D=\{(x, 0): x \in \mathbb{R}\}$ é o conjunto de descontinuidade.

Temos que descrever tal fenômeno, ou seja, uma família de campos vetoriais que possui uma singularidade cuja ordenada vai diminuindo de acordo com o parâmetro, e se anula quando este se anular. Porém, as singularidades têm comportamentos distintos em cada capítulo, ou seja, é um foco nos capítulos 3 e 4, uma sela no capítulo 5, um nó no capítulo 6. Assim, o modelo mais simples que tem tais singularidades é o linear na forma

$$
X_{\lambda}(x, y)=(a x+b y+e \lambda, c x+d y+f \lambda) .
$$

É possível descobrir a expressão da singularidade dessa família de campos vetoriais, que 
apresentamos explicitamente no capítulo seguinte, e de acordo com o determinante e o traço Jacobiano da família $X_{\lambda}$, aplicado na singularidade, podemos classificá-la de acordo com o desejado.

Mas a família (2.20) não leva em consideração os termos de ordem superior em $x, y$ e $\lambda$. Para estudarmos esse caso mais geral, vamos considerar os restos de ordem superior e verificar que tais restos não interferem, ou seja, se anulam em partes compactas. Vamos descrever o campo não linear que iremos trabalhar e a prova do fato acima na próxima subseção.

\subsubsection{Eliminação do resto}

Como dissemos no início desta seção, vamos trabalhar com uma família a um parâmetro de campos vetoriais não linear que tenha uma singularidade, para valores negativos e pequenos do parâmetro, que colida com o conjunto de descontinuidade na origem quando o parâmetro se anula. Assim, considerando a expansão em séries de Taylor em uma vizinhança da origem temos a seguinte família para $X_{\lambda}$ definida em $N$

$$
X_{\lambda}(x, y)=\left(a x+b y+e \lambda+R_{X}(x, y, \lambda), c x+d y+f \lambda+S_{X}(x, y, \lambda)\right)
$$

onde $R_{X}(x, y, \lambda)$ e $S_{X}(x, y, \lambda)$ são restos com termos de ordem maior ou igual a 2 em $x, y$ e $\lambda$, ou seja,

$$
\begin{aligned}
& R_{X}(x, y, \lambda)=a_{200} x^{2}+a_{020} y^{2}+a_{002} \lambda^{2}+a_{110} x y+a_{101} x \lambda+a_{011} y \lambda+\cdots \\
& S_{X}(x, y, \lambda)=b_{200} x^{2}+b_{020} y^{2}+b_{002} \lambda^{2}+b_{110} x y+b_{101} x \lambda+b_{011} y \lambda+\cdots
\end{aligned}
$$

onde $a_{i j k}$ e $b_{i j k}$, para $i, j, k \in \mathbb{N}$ tais que $i+j+k \geq 2$, são constantes.

Note que o fato da singularidade da família $X_{\lambda}$ colidir com a origem quando o parâmetro se anula, nos diz que os termos constantes da expansão de $X_{\lambda}$ são nulos, por isto temos uma expressão linear mais um resto de termos com ordem superior.

Uma vez que temos a expressão da família $X_{\lambda}$, a expressão da família $Y_{\lambda}$ em $S$ que consideraremos é $Y_{\lambda}(x, y)=(\alpha, \beta)$ onde $\alpha$ e $\beta$ são números reais.

Agora que temos as expressões das famílias $X_{\lambda}$ e $Y_{\lambda}$, precisamos de um resultado que garanta que os restos não irão interferir na família regularizada, ou seja, vamos provar que em partes compactas os restos vão a zero quando o parâmetro de regularização vai a zero. Para isto, lembramos que o conjunto $M$ é dado pela função $F(x, y)=y$ e que dado $\varepsilon>0$, a 
função de transição tem a seguinte expressão

$$
\varphi_{\varepsilon}(F(x, y))=\varphi_{\varepsilon}(y)=\frac{1}{2}+\frac{y}{2 \sqrt{y^{2}+\varepsilon^{2}}}
$$

para todo ponto $(x, y) \in M$.

Proposição 8. Seja $Z_{\lambda}=\left(X_{\lambda}, Y_{\lambda}\right)$ uma $F C D$ a um parâmetro real, $\lambda$, definida em $M$. Sejam $X_{\lambda}$ e $Y_{\lambda}$ dados por

$$
\begin{aligned}
& X_{\lambda}(x, y)=\left(a x+b y+e \lambda+R_{X}(x, y, \lambda), c x+d y+f \lambda+S_{X}(x, y, \lambda)\right) \\
& Y_{\lambda}(x, y)=(\alpha, \beta)
\end{aligned}
$$

onde $a, b, c, d, e, f, \alpha$ e $\beta$ são valores reais, $R_{X}(x, y, \lambda)$ e $S_{X}(x, y, \lambda)$ são os restos dados por (2.22). Sejam $\varepsilon>0$ e $\varphi_{\varepsilon}(y)$ a função de transição, então a familia de campos vetoriais regularizada, $Z_{\lambda, \varepsilon}$, é tal que os restos $R_{X}$ e $S_{X}$ se anulam quando o parâmetro de regularização tende a zero.

Demonstração. Seja $Z_{\lambda}=\left(X_{\lambda}, Y_{\lambda}\right)$ a FCD dada por (2.24) definida no subconjunto compacto $M \subseteq \mathbb{R}^{2}$. Seja $\varepsilon>0$, utilizando a função de transição dada por (2.23) e a fórmula que nos dá a expressão da família regularizada obtemos

$$
Z_{\lambda, \varepsilon}(x, y)=\left(Z_{\lambda, \varepsilon}^{1}(x, y), Z_{\lambda, \varepsilon}^{2}(x, y)\right)
$$

onde

$$
\begin{aligned}
Z_{\lambda, \varepsilon}^{1}(x, y) & =\left(\frac{1}{2}-\frac{y}{2 \sqrt{y^{2}+\varepsilon^{2}}}\right) \alpha+\left(\frac{1}{2}+\frac{y}{2 \sqrt{y^{2}+\varepsilon^{2}}}\right)(a x+b y \\
& \left.+e \lambda+R_{X}(x, y, \lambda)\right) \\
Z_{\lambda, \varepsilon}^{2}(x, y) & =\left(\frac{1}{2}-\frac{y}{2 \sqrt{y^{2}+\varepsilon^{2}}}\right) \beta+\left(\frac{1}{2}+\frac{y}{2 \sqrt{y^{2}+\varepsilon^{2}}}\right)(c x+d y \\
& \left.+f \lambda+S_{X}(x, y, \lambda)\right) .
\end{aligned}
$$

Agora, vamos efetuar a seguinte mudança de variáveis e reescalonamento de parâmetros nas duas componentes de $Z_{\lambda, \varepsilon}$

$$
x=\varepsilon \widetilde{x}, \quad y=\varepsilon \widetilde{y}, \quad \lambda=\varepsilon \widetilde{\lambda}, \quad \alpha=\varepsilon \widetilde{\alpha}, \quad \beta=\varepsilon \widetilde{\beta} .
$$

Assim, após essa mudança de variáveis e reescalonamento dos parâmetros, as duas com- 
ponentes da família regularizada são reduzidas a

$$
\begin{aligned}
\varepsilon \widetilde{x}^{\prime} & =\left(\frac{1}{2}-\frac{\widetilde{y}}{2 \sqrt{(\widetilde{y})^{2}+1}}\right) \varepsilon \widetilde{\alpha}+\left(\frac{1}{2}+\frac{\widetilde{y}}{2 \sqrt{(\widetilde{y})^{2}+1}}\right)(a \varepsilon \widetilde{x}+b \varepsilon \widetilde{y} \\
& \left.+e \varepsilon \widetilde{\lambda}+R_{X}(\varepsilon \widetilde{x}, \varepsilon \widetilde{y}, \varepsilon \widetilde{\lambda})\right), \\
\varepsilon \widetilde{y}^{\prime} & =\left(\frac{1}{2}-\frac{\widetilde{y}}{2 \sqrt{(\widetilde{y})^{2}+1}}\right) \varepsilon \widetilde{\beta}+\left(\frac{1}{2}+\frac{\widetilde{y}}{2 \sqrt{(\widetilde{y})^{2}+1}}\right)(c \varepsilon \widetilde{x}+d \varepsilon \widetilde{y} \\
& \left.+f \varepsilon \widetilde{\lambda}+S_{X}(\varepsilon \widetilde{x}, \varepsilon \widetilde{y}, \varepsilon \widetilde{\lambda})\right),
\end{aligned}
$$

onde

$$
\begin{aligned}
R_{X}(\varepsilon \widetilde{x}, \varepsilon \widetilde{y}, \varepsilon \widetilde{\lambda}) & =a_{200} \varepsilon^{2}(\widetilde{x})^{2}+a_{020} \varepsilon^{2}(\widetilde{y})^{2}+a_{002} \varepsilon^{2}(\widetilde{\lambda})^{2}+a_{110} \varepsilon^{2} \widetilde{x} \widetilde{y}+a_{101} \varepsilon^{2} \widetilde{x} \widetilde{\lambda} \\
& +a_{011} \varepsilon^{2} \widetilde{y} \widetilde{\lambda}+\cdots \\
S_{X}(\varepsilon \widetilde{x}, \varepsilon \widetilde{y}, \varepsilon \widetilde{\lambda}) & =b_{200} \varepsilon^{2}(\widetilde{x})^{2}+b_{020} \varepsilon^{2}(\widetilde{y})^{2}+b_{002} \varepsilon^{2}(\widetilde{\lambda})^{2}+b_{110} \varepsilon^{2} \widetilde{x} \widetilde{y}+b_{101} \varepsilon^{2} \widetilde{x} \widetilde{\lambda} \\
& +b_{011} \varepsilon^{2} \widetilde{y} \widetilde{\lambda}+\cdots .
\end{aligned}
$$

Portanto, é fácil ver que podemos colocar $\varepsilon$ em evidência em qualquer um dos restos acima, donde colocamos $\varepsilon$ em evidência do lado direito das igualdades (2.26) que definem as componentes da família regularizada. Assim, cancelando o $\varepsilon$ do lado esquerdo com o do lado direito das igualdades em (2.26) obtemos

$$
\begin{aligned}
\widetilde{x}^{\prime} & =\left(\frac{1}{2}-\frac{\widetilde{y}}{2 \sqrt{(\widetilde{y})^{2}+1}}\right) \widetilde{\alpha}+\left(\frac{1}{2}+\frac{\widetilde{y}}{2 \sqrt{(\widetilde{y})^{2}+1}}\right)(a \widetilde{x}+b \widetilde{y} \\
& \left.+e \widetilde{\lambda}+\widetilde{R}_{X}(\varepsilon \widetilde{x}, \varepsilon \widetilde{y}, \varepsilon \widetilde{\lambda})\right), \\
\widetilde{y}^{\prime} & =\left(\frac{1}{2}-\frac{\widetilde{y}}{2 \sqrt{(\widetilde{y})^{2}+1}}\right) \widetilde{\beta}+\left(\frac{1}{2}+\frac{\widetilde{y}}{2 \sqrt{(\widetilde{y})^{2}+1}}\right)(c \widetilde{x}+d \widetilde{y} \\
& \left.+f \widetilde{\lambda}+\widetilde{S}_{X}(\varepsilon \widetilde{x}, \varepsilon \widetilde{y}, \varepsilon \widetilde{\lambda})\right),
\end{aligned}
$$


onde $\widetilde{R}_{X}$, e $\widetilde{S}_{X}$ são os restos dados por

$$
\begin{aligned}
\widetilde{R}_{X}(\varepsilon \widetilde{x}, \varepsilon \widetilde{y}, \varepsilon \widetilde{\lambda}) & =a_{200} \varepsilon(\widetilde{x})^{2}+a_{020} \varepsilon(\widetilde{y})^{2}+a_{002} \varepsilon(\widetilde{\lambda})^{2}+a_{110} \varepsilon \widetilde{x} \widetilde{y}+a_{101} \varepsilon \widetilde{x} \widetilde{\lambda} \\
& +a_{011} \varepsilon \widetilde{y} \widetilde{\lambda}+\cdots \\
\widetilde{S}_{X}(\varepsilon \widetilde{x}, \varepsilon \widetilde{y}, \varepsilon \widetilde{\lambda}) & =b_{200} \varepsilon(\widetilde{x})^{2}+b_{020} \varepsilon(\widetilde{y})^{2}+b_{002} \varepsilon(\widetilde{\lambda})^{2}+b_{110} \varepsilon \widetilde{x} \widetilde{y}+b_{101} \varepsilon \widetilde{x} \widetilde{\lambda} \\
& +b_{011} \varepsilon \widetilde{y} \widetilde{\lambda}+\cdots .
\end{aligned}
$$

Assim, tomando o limite dos restos quando $\varepsilon$ vai a zero, por valores positivos, é fácil ver que

$$
\begin{aligned}
& \lim _{\varepsilon \rightarrow 0} \widetilde{R}_{X}(\varepsilon \widetilde{x}, \varepsilon \widetilde{y}, \varepsilon \widetilde{\lambda})=0 \\
& \lim _{\varepsilon \rightarrow 0} \widetilde{S}_{X}(\varepsilon \widetilde{x}, \varepsilon \widetilde{y}, \varepsilon \widetilde{\lambda})=0 .
\end{aligned}
$$

Portanto, ao invés de considerarmos uma família a dois parâmetros, a saber $\varepsilon$ e $\lambda$, consideramos a família a um parâmetro, $\lambda$, dada por

$$
\begin{aligned}
& \widetilde{x}^{\prime}=\left(\frac{1}{2}-\frac{\widetilde{y}}{2 \sqrt{(\widetilde{y})^{2}+1}}\right) \widetilde{\alpha}+\left(\frac{1}{2}+\frac{\widetilde{y}}{2 \sqrt{(\widetilde{y})^{2}+1}}\right)(a \widetilde{x}+b \widetilde{y}+e \widetilde{\lambda}), \\
& \widetilde{y}^{\prime}=\left(\frac{1}{2}-\frac{\widetilde{y}}{2 \sqrt{(\widetilde{y})^{2}+1}}\right) \widetilde{\beta}+\left(\frac{1}{2}+\frac{\widetilde{y}}{2 \sqrt{(\widetilde{y})^{2}+1}}\right)(c \widetilde{x}+d \widetilde{y}+f \widetilde{\lambda}) .
\end{aligned}
$$

Assim, pela proposição anterior, quando formos estudar as bifurcações que ocorrem nas famílias regularizadas cuja respectiva FCD possui uma singularidade que colide com o conjunto de descontinuidade, consideraremos a seguinte família de campos vetoriais descontínuos $Z_{\lambda}(x, y)=\left(X_{\lambda}(x, y), Y_{\lambda}(x, y)\right)$ onde

$$
\begin{aligned}
X_{\lambda}(x, y) & =(a x+b y+e \lambda, c x+d y+f \lambda) \\
Y_{\lambda}(x, y) & =(\alpha, \beta) .
\end{aligned}
$$

Obs.: Na tentativa de tornar o resultado anterior mais geral, poderíamos considerar que a família $Y_{\lambda}$ fosse dada por

$$
Y_{\lambda}(x, y)=\left(\alpha+R_{Y}(x, y, \lambda), \beta+S_{Y}(x, y, \lambda)\right)
$$

onde $R_{Y}(x, y, \lambda)$ e $S_{Y}(x, y, \lambda)$ são restos com termos de ordem maior ou igual a $1 \mathrm{em} x, y$ e 
$\lambda$, ou seja,

$$
\begin{aligned}
R_{Y}(x, y, \lambda) & =c_{100} x+c_{010} y+c_{001} \lambda+c_{200} x^{2}+c_{020} y^{2}+c_{002} \lambda^{2}+c_{110} x y+c_{101} x \lambda \\
& +c_{011} y \lambda+\cdots \\
S_{Y}(x, y, \lambda) & =d_{100} x+d_{010} y+d_{001} \lambda+d_{200} x^{2}+d_{020} y^{2}+d_{002} \lambda^{2}+d_{110} x y+d_{101} x \lambda \\
& +d_{011} y \lambda+\cdots,
\end{aligned}
$$

onde $c_{i j k}$ e $d_{i j k}$, para $i, j, k \in \mathbb{N}$ tais que $i+j+k \geq 1$, são constantes. Porém, não é possível mostrar que os restos $R_{Y}$ e $S_{Y}$ se anulam com o parâmetro da regularização. Isto se deve à presença dos termos lineares em $x, y$ e $\lambda$. Assim, se consideramos inicialmente que $c_{100}=c_{010}=c_{001}=0$ e que $d_{100}=d_{010}=d_{001}=0$ então, neste caso, teremos que o resultado anterior vale para essa nova família $Y_{\lambda}(x, y)=\left(\alpha+R_{Y}(x, y, \lambda), \beta+S_{Y}(x, y, \lambda)\right)$ com os restos possuindo termos de ordem maior ou igual a dois em $x, y$ e $\lambda$.

\subsubsection{Mudança de variáveis}

Vamos apresentar uma mudança de variáveis que nos auxiliará na simplificação da família regularizada associada a (2.32). Tal mudança de variáveis transforma a família de campos vetoriais $\widetilde{Y}_{\lambda}=(\alpha, \beta)$ em $Y_{\lambda}=(0,1)$, além disso o conjunto de descontinuidade é invariante por essa mudança, e essa mudança de variáveis leva a família de campos $\widetilde{X}_{\tilde{\lambda}}=(\widetilde{a} \widetilde{x}+\widetilde{b} \widetilde{y}+$ $\widetilde{e} \widetilde{\lambda}, \widetilde{c x}+\widetilde{d} \widetilde{y}+\widetilde{f} \lambda)$ em $X_{\lambda}=(a x+b y+e \lambda, c x+d y+f \lambda)$.

Definição 14. Sejam $\varphi_{1}$ e $\varphi_{2}$ os fluxos gerados pelos campos $X: U_{1} \subset \mathbb{R}^{2} \rightarrow \mathbb{R}^{2}$ e $Y: U_{2} \subset$ $\mathbb{R}^{2} \rightarrow \mathbb{R}^{2}$, respectivamente. Diz-se que $X$ é topologicamente conjugado (resp. $C^{r}$-conjugado) a $Y$ quando existe um homeomorfismo (resp. um difeomorfismo de classe $C^{r}$ ) $h: U_{1} \rightarrow U_{2}$ tal que $h\left(\varphi_{1}(t, x)\right)=\varphi_{2}(t, h(x))$ para todo $(t, x) \in \mathbb{R} \times U_{1}$.

Agora, vamos encontar um difeomorfismo que conjuga o campo $\widetilde{Y}_{\lambda}=(\alpha, \beta)$ ao campo $Y_{\lambda}=(1,0)$.

Lema 9. Seja $\widetilde{Y}_{\lambda}(x, y)=(\alpha, \beta)$ um campo vetorial de classe $C^{r}, r \geq 1$, com $\beta \neq 0$. Então, o campo $\widetilde{Y}_{\lambda}$ é $C^{r}$ - conjugado ao campo $Y_{\lambda}(x, y)=(1,0)$.

Demonstração. Sejam $\widetilde{Y}_{\lambda}(x, y)=(\alpha, \beta) \operatorname{com} \beta \neq 0$ um campo vetorial de classe $C^{r}(r \geq 1)$ definido em $M \subseteq \mathbb{R}^{2}$ e $p=(0,0)$ um ponto regular de $Y_{\lambda}(x, y)=(1,0)$. Seja $D=\{(t, u)$ : $t \in \mathbb{R}, u \in M\}$, o fluxo de $\widetilde{Y}$ é dado por $\psi: D \rightarrow M$ onde

$$
\psi\left(t,\left(u_{1}, u_{2}\right)\right)=\left(\alpha t+u_{1}, \beta t+u_{2}\right)
$$


onde $t \in \mathbb{R}$ e $\left(u_{1}, u_{2}\right) \in \mathbb{R}^{2}$.

Vamos supor que $\{y=0\}$ é a seção transversal $\Sigma$, ou seja, $\Sigma$ é dada pela função $f: A \subseteq$ $\mathbb{R} \rightarrow \mathbb{R}^{2}$ dada por $f(x)=(x, 0)$. Além disso, $f(0)=(0,0)=p$.

Sejam $D_{A}=\{(t, v):(t, f(v))=(t, v, 0) \in D\} \subset \mathbb{R}^{2}$ e $F: D_{A} \rightarrow M$ dada por

$$
F(t, v)=\psi(t, f(v))=\psi(t, v, 0)=(\alpha t+v, \beta t)
$$

Como consequência da prova do teorema do fluxo tubular, veja [S2], temos que a aplicação $F$ é um difeomorfismo local, de classe $C^{r}$, definido em $(-\varepsilon, \varepsilon) \times B$ onde $B$ é um intervalo aberto centrado na origem de $\mathbb{R}$, e $\varepsilon>0$. Além disso, se $V=F((-\varepsilon, \varepsilon) \times B)$ então a conjugação entre os campos $\widetilde{Y}$ e $Y$ é dada pela função $H: V \rightarrow(-\varepsilon, \varepsilon) \times B$ definida por

$$
H=\left.F^{-1}\right|_{(-\varepsilon, \varepsilon) \times B} .
$$

Agora como o determinante Jacobiano de $F$ é sempre igual a $-\beta \neq 0$, então pelo teorema da função inversa temos que $D F^{-1}(p)=D H(p)$. Logo,

$$
D H(p)=-\frac{1}{\beta}\left(\begin{array}{cc}
0 & -1 \\
-\beta & \alpha
\end{array}\right)
$$

e daí tiramos que se $H=\left(H_{1}, H_{2}\right)$ então

$$
\begin{aligned}
\frac{\partial H_{1}}{\partial t} & =0, & \frac{\partial H_{1}}{\partial v} & =\frac{1}{\beta} \\
\frac{\partial H_{2}}{\partial t} & =1, & \frac{\partial H_{2}}{\partial v} & =-\frac{\alpha}{\beta}
\end{aligned}
$$

portanto, $H_{1}(t, v)=v / \beta$, e $H_{2}(t, v)=t-\alpha v / \beta$, ou seja, a função que conjuga os campos $\widetilde{Y}$ e $Y$ é dada por

$$
H(t, v)=\left(\frac{v}{\beta}, t-\frac{\alpha}{\beta} v\right) .
$$

Uma vez que temos esse lema, fica fácil encontrarmos o difeormorfismo que queremos.

Proposição 10. Seja $\widetilde{Y}_{\lambda}(x, y)=(\alpha, \beta)$ um campo vetorial de classe $C^{r}, r \geq 1$, com $\beta \neq 0$. Então, o campo $\widetilde{Y}_{\lambda}$ é $C^{r}$-conjugado ao campo $\widehat{Y}_{\lambda}(x, y)=(0,1)$. 
Demonstração. Pelo lema 9 temos que o difeomorfismo $H(t, v)=\left(\frac{v}{\beta}, t-\frac{\alpha}{\beta} v\right)$ conjuga as famílias de campos $\widetilde{Y}_{\lambda}=(\alpha, \beta)$ e $Y_{\lambda}=(1,0)$. Por outro lado, a rotação de ângulo $\pi$ leva a família de campos $Y_{\lambda}=(1,0)$ em $\widehat{Y}_{\lambda}=(0,1)$. Portanto a composição entre $H$ e a rotação de ângulo $\pi$ nos fornecerá o difeomorfismo que procuramos.

A mudança de variáveis $H(x, y)=(y / \beta, x-\alpha y / \beta)$ do lema anterior composta com a rotação de ângulo $\pi$, ou seja, $R_{\pi} \circ H$, é dada por

$$
\left(\begin{array}{l}
\widetilde{x} \\
\widetilde{y}
\end{array}\right)=\left(\begin{array}{cc}
0 & -1 \\
1 & 0
\end{array}\right)\left(\begin{array}{cc}
0 & \frac{1}{\beta} \\
1 & -\frac{\alpha}{\beta}
\end{array}\right)\left(\begin{array}{l}
x \\
y
\end{array}\right) .
$$

Portanto, a mudança de variáveis a ser usada é a seguinte

$$
\begin{aligned}
& \widetilde{x}=\frac{\alpha y}{\beta}-x \\
& \widetilde{y}=\frac{y}{\beta} .
\end{aligned}
$$

Assim, notamos que pela mudança de variáveis dada por (2.36), o conjunto de descontinuidade $D=\left\{(x, y) \in \mathbb{R}^{2}: y=0\right\}$ sofre apenas uma rotação de ângulo $\pi$.

Aplicando a mudança de variáveis (2.36) na família $Y_{\lambda}=(\alpha, \beta)$ obtemos $\widetilde{x}^{\prime}=(\alpha / \beta) \beta-$ $\alpha=0$ e $\widetilde{y}^{\prime}=\beta / \beta=1$. Assim, obtemos o novo campo

$$
\widetilde{Y}_{\widetilde{\lambda}}(\widetilde{x}, \widetilde{y})=(0,1)
$$

Agora, aplicando (2.36) na família

$$
\tilde{X}_{\tilde{\lambda}}(\widetilde{x}, \widetilde{y})=(\widetilde{a} \widetilde{x}+\widetilde{b} \widetilde{y}+\widetilde{e} \widetilde{\lambda}, \widetilde{c} \widetilde{x}+\widetilde{d} \widetilde{y}+\widetilde{f} \lambda)
$$

obtemos

$$
\begin{aligned}
& \widetilde{x}^{\prime}=\left(\frac{\widetilde{c} \alpha}{\beta}-\widetilde{a}\right) \widetilde{x}+\left(\frac{\widetilde{d} \alpha}{\beta}-\widetilde{b}\right) \widetilde{y}+\left(\frac{\widetilde{f} \alpha}{\beta}-\widetilde{e}\right) \widetilde{\lambda} \\
& \widetilde{y}^{\prime}=\frac{\widetilde{c}}{\beta} \widetilde{x}+\frac{\widetilde{d}}{\beta} \widetilde{y}+\frac{\widetilde{f}}{\beta} \widetilde{\lambda} .
\end{aligned}
$$

Substituindo os valores de $\widetilde{x}, \widetilde{y}$ e $\widetilde{\lambda}$, dados por $\widetilde{x}=-x+\alpha y, \widetilde{y}=\beta y$ e $\widetilde{\lambda}=\lambda$ obtemos, 
finalmente,

$$
\begin{aligned}
& x^{\prime}=\left(a-\frac{c \alpha}{\beta}\right) x+\left(-a \alpha+d \alpha-b \beta+\frac{c \alpha^{2}}{\beta}\right) y+\left(\frac{f \alpha}{\beta}-e\right) \lambda \\
& y^{\prime}=-\frac{c}{\beta} x+\left(\frac{c \alpha}{\beta}+d\right) y+\frac{f}{\beta} \lambda .
\end{aligned}
$$

Assim, obtemos a seguinte família

$$
X_{\lambda}(x, y)=(a x+b y+e \lambda, c x+d y+f \lambda)
$$

onde

$$
\begin{gathered}
a=\widetilde{a}-\frac{\widetilde{c} \alpha}{\beta}, \quad b=(\widetilde{d}-\widetilde{a}) \alpha-\widetilde{b} \beta+\frac{\widetilde{c} \alpha^{2}}{\beta}, \quad c=-\frac{\widetilde{c}}{\beta}, \\
d=\widetilde{d}+\frac{\widetilde{c} \alpha}{\beta}, \quad e=-\widetilde{e}+\frac{\widetilde{f} \alpha}{\beta} \quad \text { e } \quad f=\frac{\widetilde{f}}{\beta} .
\end{gathered}
$$

Resumindo, temos que a família de campos vetoriais descontínuos $\widetilde{Z}_{\widetilde{\lambda}}=\left(\widetilde{X}_{\widetilde{\lambda}}, \widetilde{Y}_{\widetilde{\lambda}}\right)$ dada por

$$
\begin{aligned}
\widetilde{X}_{\widetilde{\lambda}}(\widetilde{x}, \widetilde{y}) & =(\widetilde{a} \widetilde{x}+\widetilde{b} \widetilde{y}+\widetilde{e} \widetilde{\lambda}, \widetilde{c} \widetilde{x}+\widetilde{d} \widetilde{y}+\widetilde{f \lambda}) \\
\widetilde{Y}_{\widetilde{\lambda}}(\widetilde{x}, \widetilde{y}) & =(\alpha, \beta)
\end{aligned}
$$

é $C^{r}$-conjugada à família $Z_{\lambda}=\left(X_{\lambda}, Y_{\lambda}\right)$ dada por

$$
\begin{aligned}
& X_{\lambda}(x, y)=(a x+b y+e \lambda, c x+d y+f \lambda) \\
& Y_{\lambda}(x, y)=(0,1) .
\end{aligned}
$$

Utilizaremos essa família simplificada para apresentar um exemplo na última seção do próximo capítulo. 


\section{Capítulo 3}

\section{Bifurcação foco no bordo - parte I}

Neste capítulo iremos estudar as bifurcações que ocorrem na regularização das famílias de campos descontínuos pertencentes ao caso foco no bordo, descritos na seção 3.1.1 de [KGR]. A seção 3.1.1 do artigo citado apresenta cinco subcasos de codimensão um e cita um subcaso que tem codimensão dois. Analisaremos três subcasos, dois são de codimensão um e o terceiro, de codimensão dois, é intermediário entre os dois primeiros subcasos.

\subsection{Definição dos casos analisados}

Como foi dito no capítulo anterior, vamos considerar que $F(x, y)=y$ é a função que define $M$ e seus subconjuntos $D, N$ e $S$. Assim, o conjunto de descontinuidade, $D$, é o eixo- $x$.

Iremos estudar o caso foco no bordo levando em consideração cinco casos de codimensão 1 e um caso de codimensão 2. Grosso modo iremos descrever as bifurcações que ocorrem em uma família a um parâmetro de campos vetoriais descontínuos $Z_{\lambda}=\left(X_{\lambda}, Y_{\lambda}\right)$, quando o campo vetorial $X_{\lambda}$ possui um foco repulsor, para valores negativos e pequenos de $\lambda$, no semi-plano $\left\{(x, y) \in \mathbb{R}^{2}: y \geq 0\right\}$ e que colide com o conjunto $D$ quando $\lambda$ se anula. Para dois parâmetros a ideia é essencialmente a mesma. Vamos considerar que as órbitas do foco repulsor têm rotação horária. Os casos onde o foco é atrator e tem rotação horária ou antihorária ou o foco é repulsor com rotação anti-horária são análogos aos casos estudados aqui, bastando inverter apropriadamente as setas nas figuras 1, 2 e 3 .

Conforme a Proposição 7 da subseção 2.3.1 do capítulo anterior, consideramos os campos vetoriais a um parâmetro, $\lambda \in \mathbb{R}$, a serem estudados por

$$
\begin{aligned}
X_{\lambda}(x, y) & =(a x+b y+e \lambda, c x+d y+f \lambda) \\
Y_{\lambda}(x, y) & =(\alpha, \beta)
\end{aligned}
$$


onde $a, b, c, d, e, f$ são números reais, $\alpha \in \mathbb{R}$ e $\beta \in \mathbb{R}$.

Obs.: Na última subseção do capítulo anterior apresentamos um difeomorfismo de classe $C^{r}$ que torna as famílias (3.1) $C^{r}$-conjugadas, respectivamente, às seguintes famílias

$$
\begin{aligned}
\widetilde{X}_{\lambda}(x, y) & =(\widetilde{a} x+\widetilde{b} y+\widetilde{e} \lambda, \widetilde{c} x+\widetilde{d} y+\widetilde{f} \lambda) \\
\widetilde{Y}_{\lambda}(x, y) & =(0,1)
\end{aligned}
$$

preservando a reta de descontinuidade. As famílias (3.2) são mais simples de se trabalhar do que as famílias originais, mas isto não é de fato verdade. O modo como a nova família $\widetilde{Y}_{\lambda}$ está escrita não nos permite eliminar a variável $\varepsilon$ da regularização de (3.2), ao passo que na regularização de (3.1) isso é possível. Outro fato é que não conseguimos encontrar uma singularidade da família regularizada, associada a (3.2), que anula o determinante e o traço da matriz Jacobiana. Já na família regularizada associada a (3.1) foi possível calcular essa singularidade. Por esses dois fatos e para tornar as seções deste capítulo mais uniformes, no sentido de não considerarmos a variável $\varepsilon$ ao longo desse capítulo, iremos trabalhar com a família (3.1).

Para definir os distintos casos de foco no bordo vamos precisar dos seguintes subconjuntos do conjunto de deslizamento. O conjunto de deslizamento, $\mathcal{D}_{Z_{\lambda}}$, de $Z_{\lambda}$ é formado pelos pontos $q=(x, 0) \in D$ tais que $X F(q) Y F(q) \leq 0$, ou seja, $\mathcal{D}_{Z_{\lambda}}=\left\{(x, 0) \in \mathbb{R}^{2}: x \geq-f \lambda / c\right\}$, se $\beta>0$. Para os casos em que $\beta<0$ temos que o conjunto de deslizamento é dado por $\mathcal{D}_{Z_{\lambda}}=\left\{(x, 0) \in \mathbb{R}^{2}: x \leq-f \lambda / c\right\}$. Assim, se $\beta>0$ dado um ponto $(p, 0)$ qualquer contido no interior de $\mathcal{D}_{Z_{\lambda}}$, definimos os seguintes intervalos em $D, r_{p}=[-f \lambda / c, p)$ e $r_{\infty}=(p, \infty)$ que satisfazem $r_{p} \cup\{p\} \cup r_{\infty}=\mathcal{D}_{Z_{\lambda}}$.

Denotaremos os três subcasos de codimensão 1 do foco no bordo por $\mathcal{F}_{1}, \mathcal{F}_{2}$ e $\mathcal{F}_{3}$, o caso de codimensão dois será denotado por $\mathcal{F}_{4}$. Suas definições, de acordo com [KGR], levam em conta seus respectivos diagramas de bifurcação (veja figuras 1, 2, 3 e 4).

Definição 15. Seja $Z_{\lambda}=\left(X_{\lambda}, Y_{\lambda}\right)$ uma FCD definida em $M$ onde $Y_{\lambda}=(\alpha, \beta)$ com $\alpha \in \mathbb{R}$ e $\beta \in \mathbb{R}$. Dizemos que

a) $Z_{\lambda}$ pertence ao caso $\mathcal{F}_{1}$ se $\beta>0$ e para valores negativos e pequenos do parâmetro a família $X_{\lambda}$ possui um foco repulsor, $f_{\lambda}$, em $N$, cujas órbitas que saem do foco têm rotação horária, e uma sela, $p_{s}=\left(p_{1}, 0\right)$, do campo de Filippov em $D$. Além disso, ainda para valores negativos do parâmetro, existe um ciclo de Filippov que é formado por um arco de órbita que sai do foco, tangencia o conjunto $D$ no ponto $p_{t}=\left(p_{0}, 0\right)$ e retorna a esse conjunto no interior do intervalo $r_{p_{1}}$, definido acima, onde $p_{1}$ é a ordenada da sela 
de Filippov. Quando $\lambda=0$ o foco colide com a sela e o ponto de tangência na origem, e para valores positivos do parâmetro não existem singularidades. (O diagrama de bifurcação desse caso encontra-se na figura 1.)

b) $Z_{\lambda}$ pertence ao caso $\mathcal{F}_{2}$ se $\beta>0$ e para valores negativos e pequenos do parâmetro a família $X_{\lambda}$ possui um foco repulsor, $f_{\lambda}$, em $N$, cujas órbitas que saem do foco têm rotação horária, e uma sela, $p_{s}=\left(p_{1}, 0\right)$, do campo de Filippov em $D$. A órbita que sai do foco e tangencia o conjunto $D$ no ponto $p_{t}=\left(p_{0}, 0\right)$ e retorna a esse conjunto no intervalo $r_{\infty}$ onde, como em a), $p_{1}$ é a ordenada da sela de Filippov. Quando $\lambda=0$ o foco colide com a sela e o ponto de tangência na origem, e para valores positivos do parâmetro não existem singularidades. (Ver figura 2.)

c) $Z_{\lambda}$ pertence ao caso $\mathcal{F}_{3}$ se $\beta>0$ e para valores negativos e pequenos do parâmetro a família $X_{\lambda}$ possui um foco, $f_{\lambda}$, repulsor em $N$, cujas órbitas que saem do foco têm rotação horária, e as órbitas que encontram o conjunto de deslizamento são atraídas pelo ponto de tangência, $p_{t}$, entre uma órbita que sai do foco e o conjunto $D$. Para $\lambda=0$ o foco colide com o ponto de tangência na origem, e para valores positivos do parâmetro existe um nó, $p_{n}$, do campo de Filippov em $D$. (Ver figura 3.)

d) $Z_{\lambda}$ pertence ao caso $\mathcal{F}_{4}$ se $\beta>0$ e para valores negativos e pequenos do parâmetro a família $X_{\lambda}$ possui um foco, $f_{\lambda}$, repulsor em $N$, cujas órbitas que saem do foco têm rotação horária, e uma sela, $p_{s}=\left(p_{1}, 0\right)$, do campo de Filippov em $D$. A órbita que sai do foco e tangencia o conjunto $D$ em um ponto $p_{t}=\left(p_{0}, 0\right)$ retorna ao conjunto de descontinuidade exatamente sobre a sela de Filippov. Quando $\lambda=0$ o foco colide com a sela e o ponto de tangência na origem, e para valores positivos do parâmetro não existem singularidades. (Ver figura 4.)

e) $Z_{\lambda}$ pertence ao caso $\mathcal{F}_{5}$ se $\beta<0$ e para valores negativos e pequenos do parâmetro a família $X_{\lambda}$ possui um foco, $f_{\lambda}$, repulsor em $N$, cujas órbitas que saem do foco têm rotação horária. A órbita que sai do foco e tangencia o conjunto $D$ em um ponto $p_{t}$ retorna ao conjunto de descontinuidade em um ponto de costura. Quando $\lambda=0 \mathrm{o}$ foco colide com o ponto de tangência na origem, e para valores positivos do parâmetro existe um nó repulsor de Filippov. (Ver figura 5.)

f) $Z_{\lambda}$ pertence ao caso $\mathcal{F}_{6}$ se $\beta<0$ e para valores negativos e pequenos do parâmetro a família $X_{\lambda}$ possui um foco, $f_{\lambda}$, repulsor em $N$, cujas órbitas que saem do foco têm rotação horária, e uma sela $p_{s}$ do campo de Filippov em $D$. A órbita que sai do foco 
e tangencia o conjunto $D$ em um ponto $p_{t}$ retorna ao conjunto de descontinuidade em um ponto de costura. Quando $\lambda=0$ o foco colide com o ponto de tangência e a sela na origem, e para valores positivos do parâmetro não existem singularidades. (Ver figura 6.) 


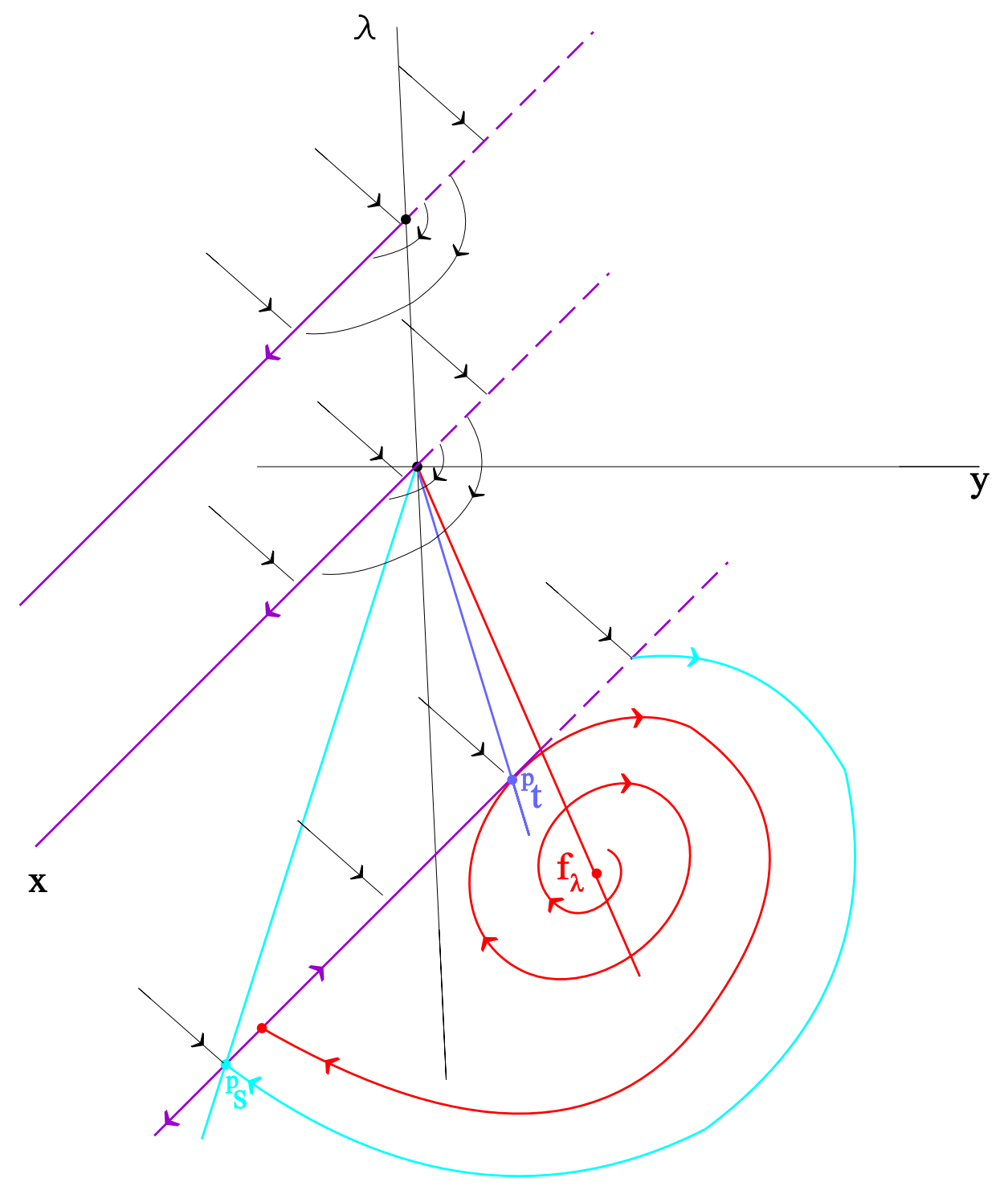

\footnotetext{
- Conjunto de deslizamento

- - - Conjunto de costura

Sela

- Foco

Ponto de tangência
}

figura 1: caso $\mathcal{F}_{1}$ 


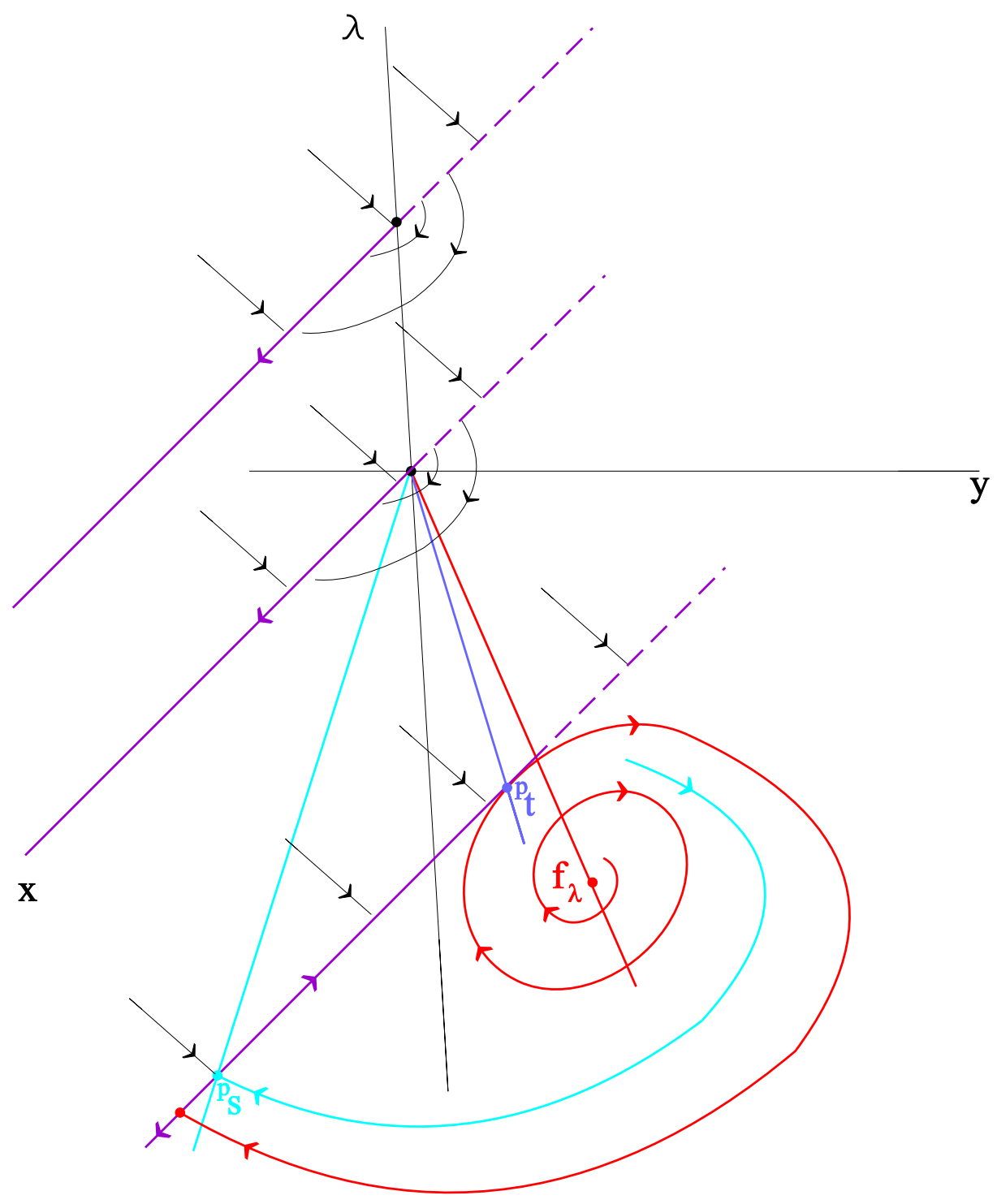

Conjunto de deslizamento

Conjunto de costura

Sela

Foco

Ponto de tangência

figura 2: caso $\mathcal{F}_{2}$ 


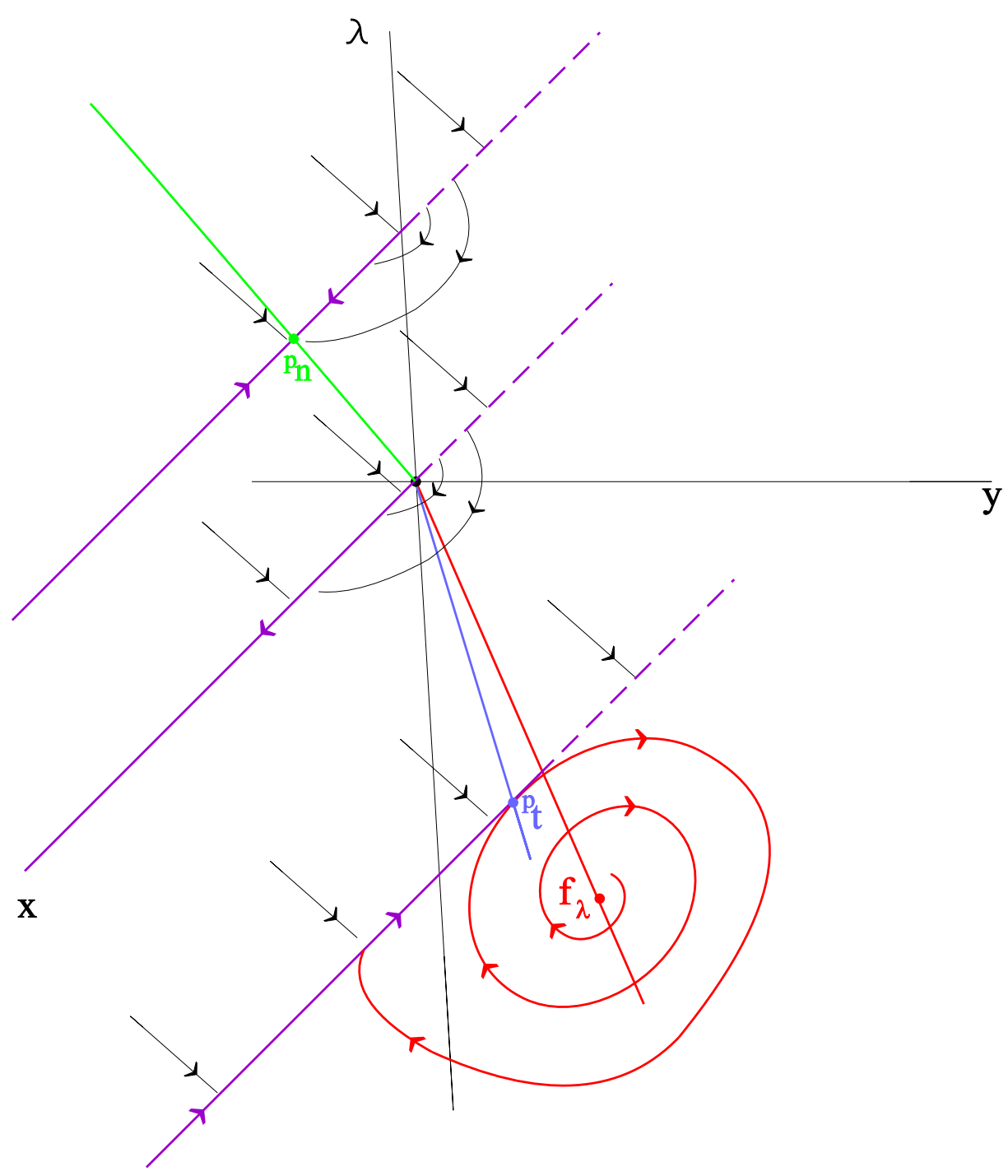

Conjunto de deslizamento

- - - Conjunto de costura

Foco

Ponto de tangência

Nó

figura 3: caso $\mathcal{F}_{3}$ 


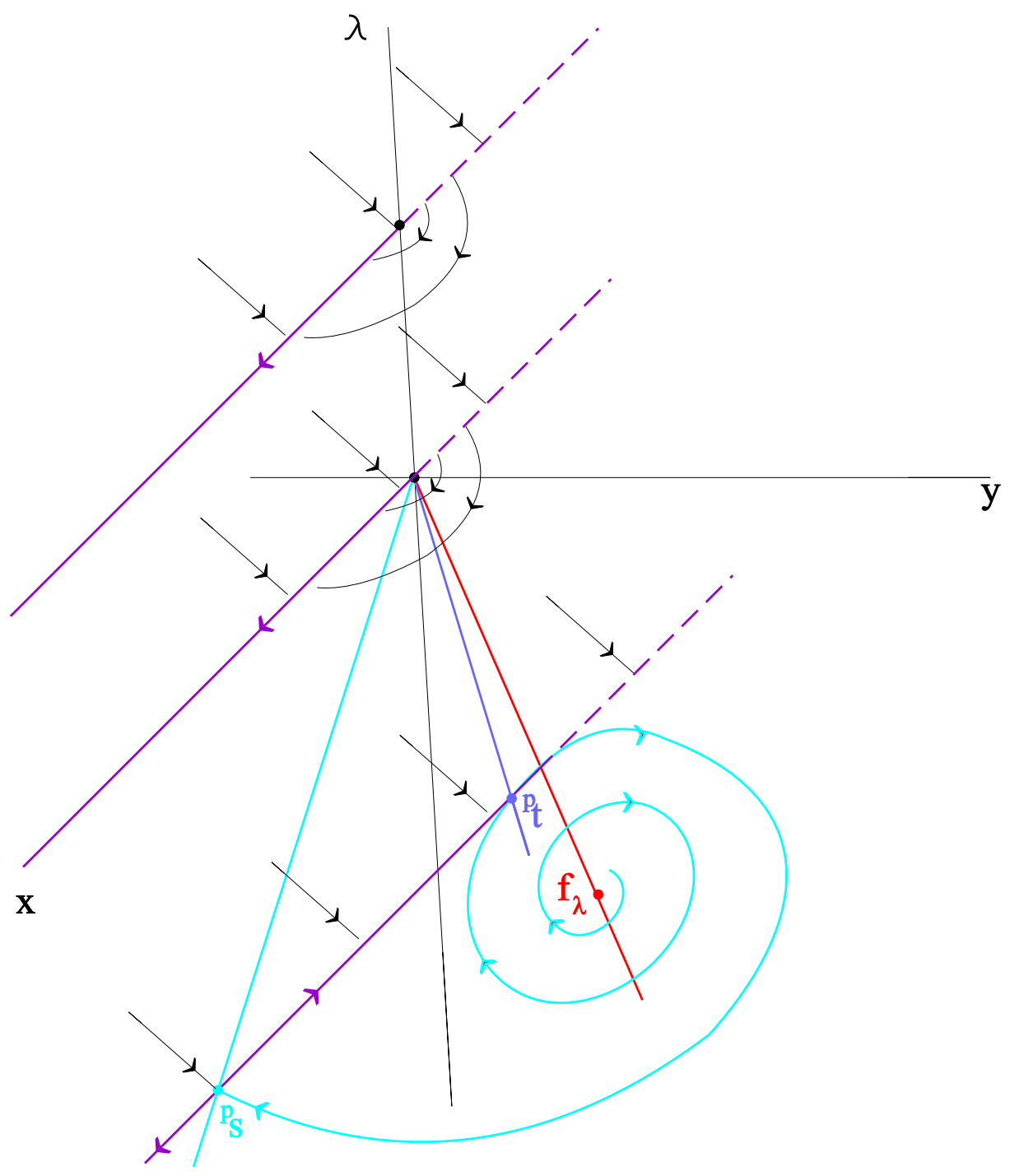

Conjunto de deslizamento

Conjunto de costura

Sela

Foco

Ponto de tangência

figura 4: caso $\mathcal{F}_{4}$ 


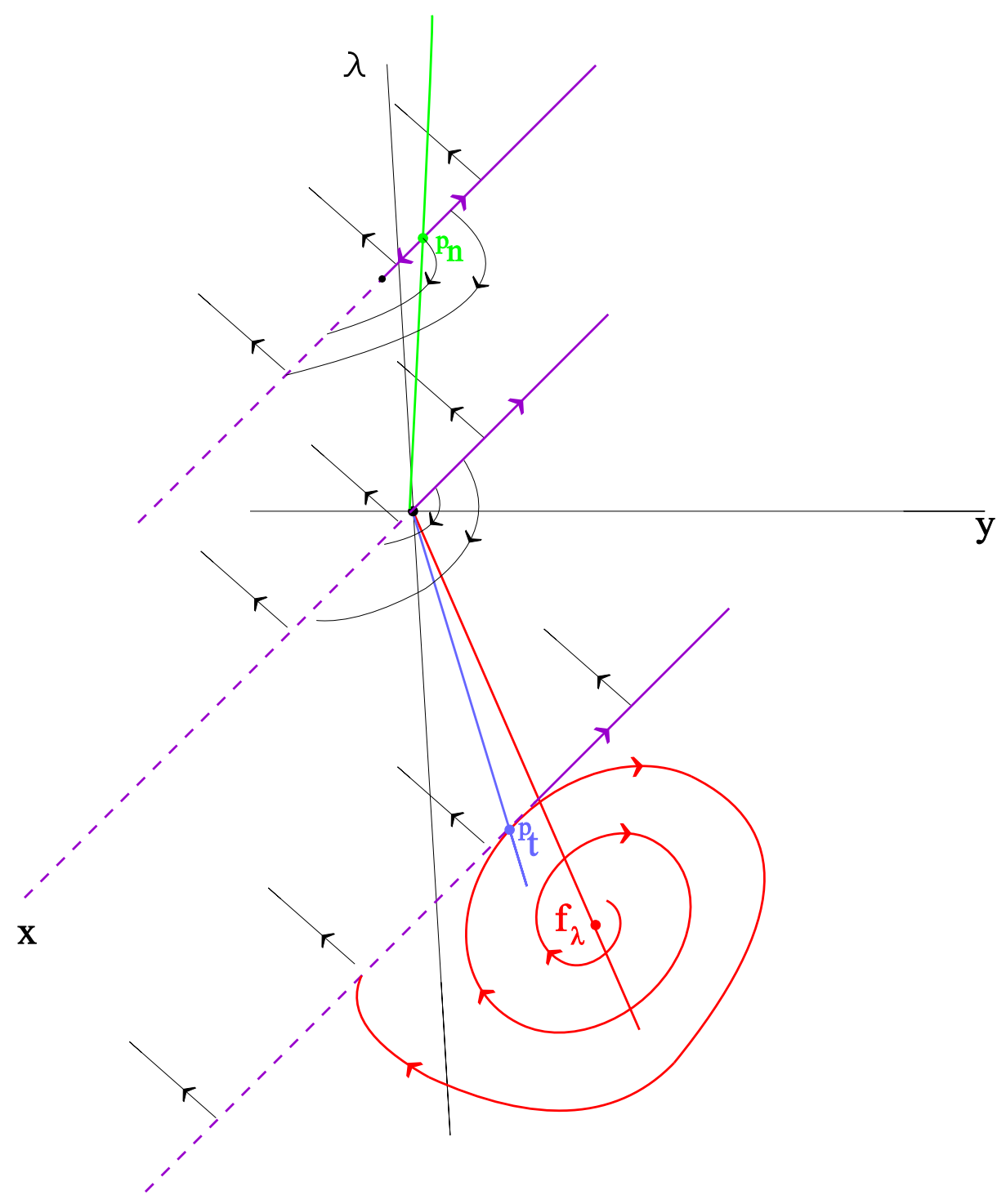

Conjunto de deslizamento

- - - Conjunto de costura

Foco

Ponto de tangência

Nó

figura 5: caso $\mathcal{F}_{5}$ 


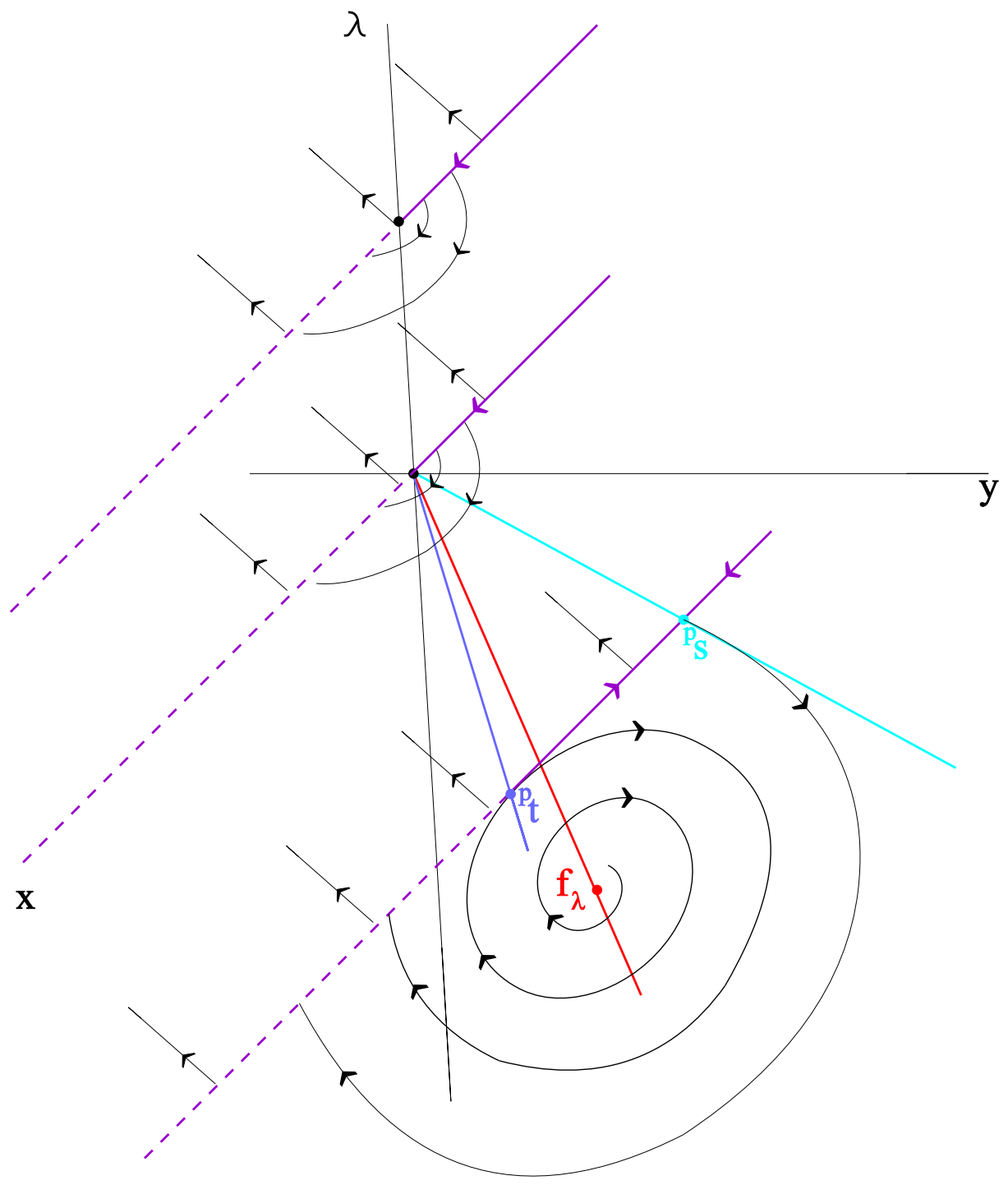

Conjunto de deslizamento

Conjunto de costura

Sela

Foco

Ponto de tangência

figura 6: caso $\mathcal{F}_{6}$ 
Considerando as definições desses casos precisamos distingui-los analiticamente. Para isso, precisaremos das seguintes considerações.

Como queremos que a família $X_{\lambda}$ tenha um foco repulsor para valores negativos e pequenos do parâmetro, cujas órbitas que saem do foco tenham rotação horária, vamos considerar as seguintes hipóteses iniciais:

H.1) $a d-b c>0$,

H.2) $a+d>0$

H.3) $(a-d)^{2}+4 b c<0$

H.4) $c<0$

onde H.1) descreve o determinante, H.2) o traço e H.3) o discriminante da parte linear do campo $X_{\lambda}$ em um ponto $(x, y)$ qualquer, ou seja, de $D X_{\lambda}(x, y)$. Além disso, H.4) está relacionado ao fato que as órbitas que saem do foco de $X_{\lambda}$ em $N$ têm rotação horária.

De H.3) e H.4) concluímos que $b$ deve ser estritamente positivo.

Voltando ao campo $X_{\lambda}$, dado por (3.1), temos que um ponto $(x, y)$ do plano é uma singularidade do campo $X_{\lambda}$ se satisfizer as seguintes relações

$$
\begin{aligned}
& x=\frac{b f-d e}{a d-b c} \lambda \\
& y=\frac{c e-a f}{a d-b c} \lambda,
\end{aligned}
$$

que estão bem definidas pois $a d-b c>0$.

Assim, se ce $-a f=0$ então a segunda coordenada da singularidade do campo $X_{\lambda}$ se anulará independentemente de $\lambda$, resultando em uma família de campos vetoriais cujas singularidades estão todas contidas no conjunto de descontinuidade. Como o foco repulsor de $X_{\lambda}$ deve aparecer em $N$ para valores pequenos e negativos do parâmetro, então devemos supor que $c e-a f<0$ pois, neste caso, a ordenada de uma singularidade de $X_{\lambda}$ será positiva. Assim, o único caso onde a origem é uma singularidade do campo $X_{\lambda}$ é quando tivermos exatamente $\lambda=0$. Portanto, nossa quinta hipótese é

H.5) $c e-a f<0$.

Um fato utilizado no próximo lema é que o ponto $p_{t}=\left(p_{0}, 0\right) \in D$ onde ocorre a tangência entre uma órbita que sai do foco repulsor e o conjunto $D$, para $\lambda<0$ pequeno, é tal que $p_{0}=-f \lambda / c$. Para verificar isto, basta notar que em $p_{t}$ a segunda componente de $X_{\lambda}$ se 
anula. Assim, como $y=0$ nesse ponto, temos que $p_{t}$ deve satisfazer $c x+f \lambda=0$. Logo, $p_{t}=(-f \lambda / c, 0)$. Além disso, da definição de $\mathcal{D}_{Z_{\lambda}}$, o ponto $p_{t}$ pertence ao bordo do conjunto de deslizamento.

O campo de Filippov, $F_{Z_{\lambda}}$, (veja $[\mathrm{M}]$ ou $[\mathrm{SM}]$ ) do campo descontínuo $Z_{\lambda}$ é dado por

$$
\begin{aligned}
F_{Z_{\lambda}}(x, y) & =l X_{\lambda}(x, y)+(1-l) Y_{\lambda}(x, y) \\
& =\left(\frac{(a \beta-c \alpha) x+(e \beta-f \alpha) \lambda}{\beta-c x-f \lambda}, 0\right)
\end{aligned}
$$

onde

$$
l=\frac{<\nabla F(x, y), Y_{\lambda}(x, y)>}{<\nabla F(x, y), Y_{\lambda}(x, y)-X_{\lambda}(x, y)>}=\frac{\beta}{\beta-c x-f \lambda} .
$$

As singularidades do campo de Filippov são pontos da forma $(x, 0)$ onde

$$
x=\frac{e \beta-f \alpha}{c \alpha-a \beta} \lambda .
$$

Será provado no próximo lema que os casos $\mathcal{F}_{1}, \mathcal{F}_{2}$ e $\mathcal{F}_{4}$ têm em comum o fato de termos $c \alpha-$ $a \beta>0$, ao passo que no caso $\mathcal{F}_{3}$ temos que $c \alpha-a \beta<0$. Assim, usando essas desigualdades temos que para os casos $\mathcal{F}_{1}, \mathcal{F}_{2}$ e $\mathcal{F}_{4}$ as singularidades do campo de Filippov estão contidas no conjunto de deslizamento, e no caso $\mathcal{F}_{3}$ não existe singularidade. Verificaremos na penúltima seção deste capítulo como identificar quando uma FCD, $Z_{\lambda}$, pertence aos casos $\mathcal{F}_{5}$ e $\mathcal{F}_{6}$.

Iremos considerar nos subcasos de foco no bordo que $\alpha>0$, isto se deve ao fato que quando $\alpha<0$ temos que o conjunto de deslizamento não vira de um conjunto de escape variando o valor de $\alpha$, mas isto poderia acontecer considerando $\alpha>0$. Portanto, vamos estudar o caso menos trivial.

Lema 11. Seja $Z_{\lambda}=\left(X_{\lambda}, Y_{\lambda}\right)$ uma família a 1-parâmetro de campos vetoriais descontínuos dada por (3.1) tal que $a d-b c>0, a+d>0,(a-d)^{2}+4 b c<0, c<0$ e ce $-a f<0$.

a) Se $\alpha>a \beta / c, a>0, a \neq d$ e $G(a, b, c, d)=0$, onde $G: \mathbb{R}^{4} \rightarrow \mathbb{R}$ é a função dada por

$$
G(a, b, c, d)=\frac{a+d}{\Delta} \operatorname{arctg}\left(\frac{\Delta}{d-a}\right)+\ln \left(\frac{a}{\sqrt{-b c}}\right)
$$

onde $\Delta=\sqrt{-(a-d)^{2}-4 b c}$, então o campo $Z_{\lambda}$ é do tipo $\mathcal{F}_{4}$.

b) Sejam $\alpha>a \beta / c, a>0$ e $a \neq d$.

b.1) Se $G(a, b, c, d)<0$ então o campo $Z_{\lambda}$ é do tipo $\mathcal{F}_{1}$. 
b.2) Se $G(a, b, c, d)>0$ então o campo $Z_{\lambda}$ é do tipo $\mathcal{F}_{2}$.

c) Se $\alpha<a \beta /$ c e $a<0$ então o campo $Z_{\lambda}$ é do tipo $\mathcal{F}_{3}$.

Demonstração. Distinguimos os casos das figuras 1 e 2 pela órbita de $p_{t}$, para $\lambda<0$ pequeno. Nos casos $\mathcal{F}_{1}$ e $\mathcal{F}_{2}$ o ponto $p_{s}$ é uma sela do campo de Filippov, cujo conjunto estável em $N$ chega perpendicularmente em $D$. No caso $\mathcal{F}_{1}$ a órbita de $p_{t}$ retorna a $D$ à esquerda de $p_{s}$, e no caso $\mathcal{F}_{2}$ à direita. Agora, para distinguirmos os casos $\mathcal{F}_{1}$ e $\mathcal{F}_{2}$ do caso $\mathcal{F}_{3}$, devemos impor que ao passo que os casos $\mathcal{F}_{1}$ e $\mathcal{F}_{2}$ têm singularidades em $D_{Z_{\lambda}}$, para valores negativos e pequenos do parâmetro, o caso $\mathcal{F}_{3}$ não tem nenhuma singularidade nesse conjunto. Além disso, no caso $\mathcal{F}_{3}$ devemos levar em consideração que as isóclinas onde o primeiro termo do campo $X_{\lambda}$ se anula são retas com coeficiente angular positivo.

Em $p_{s}=\left(p_{1}, 0\right)$ o campo $X_{\lambda}$ se anula na primeira coordenada, ou seja, $a p_{1}+e \lambda=0$ implicando que $p_{1}=-e \lambda / a$. Assim, se a órbita de $p_{t}$ retorna ao conjunto de descontinuidade entre $p_{t}$ e $p_{s}$ então $Z_{\lambda}$ está relacionado ao caso $\mathcal{F}_{1}$, se retorna depois de $p_{s}$ então $Z_{\lambda}$ está relacionado ao caso $\mathcal{F}_{2}$ e, por fim, se o ponto de interseção é $p_{s}$ então $Z_{\lambda}$ está relacionado ao caso $\mathcal{F}_{4}$.

Voltando aos casos $\mathcal{F}_{1}, \mathcal{F}_{2}$ e $\mathcal{F}_{3}$ apresentaremos uma caracterização mais completa no sentido de envolver termos dos campos $X_{\lambda}$ e $Y_{\lambda}$.

Da fórmula (3.5), vamos considerar a seguinte função de $\mathbb{R}$ em $\mathbb{R}$

$$
R(\alpha)=\frac{e \beta-f \alpha}{c \alpha-a \beta} .
$$

A função $R(\alpha)$ é uma função racional de primeiro grau, ou seja, $R(\alpha)=\frac{A \alpha+B}{C \alpha+D}$ onde $A=-f, B=e \beta, C=c$ e $D=-a \beta$. Temos que $A D-B C=-(c e-a f) \beta$ e como $\beta>0$ e $c e-a f<0$ segue que $A D-B C>0$. Logo, o gráfico de $R(\alpha)$ é uma hipérbole com assíntota vertical em $\alpha=a \beta / c$ e assíntota horizontal $-f / c$. O sinal de $A D-B C$ informa em qual semi-plano, delimitado pela assíntota vertical, a hipérbole é côncava ou convexa. No nosso caso, como $A D-B C>0$ então a parte da hipérbole que está a direita da assíntota vertical é côncava.

Considerando o plano $(\lambda, x)$ temos então que os valores da abscissa das singularidades do campo de Filippov pertencem à reta $s_{x}$ dada por $x=R(\alpha) \lambda$. Assim, para valores de $\alpha$ menores que $a \beta / c$ a reta $s_{x}$ tem inclinação maior que $-f / c$, e se $\alpha>a \beta / c$ então a inclinação da reta $s_{x}$ é menor que $-f / c$. Portanto, o campo de Filippov terá singularidades no conjunto de deslizamento se $\alpha>a \beta / c$. Resumindo, temos que se $\alpha<a \beta / c$ então as singularidades do 


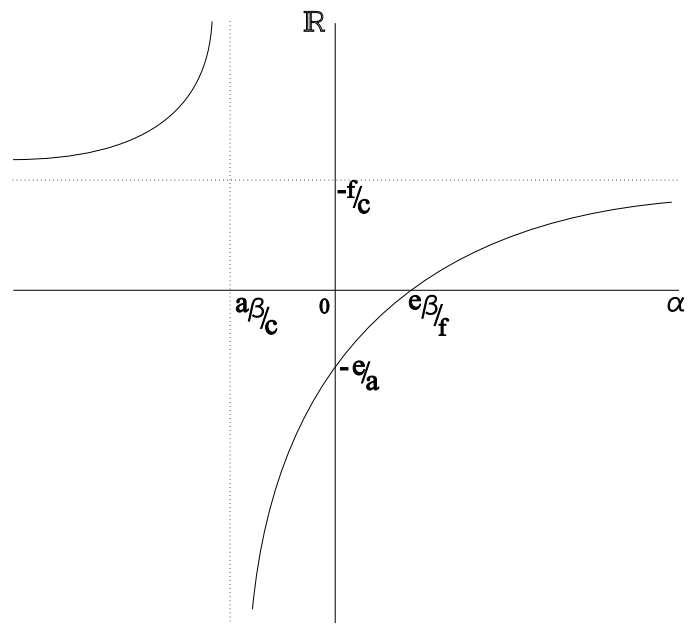

figura 7: gráfico de $R(\alpha)$

campo de Filippov estão à esquerda do conjunto de deslizamento, caracterizando o caso $\mathcal{F}_{3}$, e se ocorre a desigualdade oposta para $\alpha$ então temos a caracterização dos casos $\mathcal{F}_{1}$ e $\mathcal{F}_{2}$.

Ainda com relação ao caso $\mathcal{F}_{3}$, notamos que da sua definição devemos ter que as isóclinas que anulam o primeiro termo do campo $X_{\lambda}$ devem ter coeficiente angular maior ou igual a zero. Essas isóclinas são retas dadas por

$$
y=-\frac{a}{b} x-\frac{e}{b} \lambda
$$

Se o coeficiente angular dessas retas for zero então $a=0$, mas teríamos $\alpha<a \beta / c=0$ o que contradiz a hipótese de que $\alpha>0$. Como $b>0$, para que o coeficiente angular da isóclina seja estritamente positivo devemos ter $-a / b>0$, implicando que $a<0$. Portanto, o caso $\mathcal{F}_{3}$ está caracterizado pelas condições $a<0$ e $\alpha<a \beta / c$.

Agora, vamos distinguir os casos $\mathcal{F}_{1}$ e $\mathcal{F}_{2}$. Para isso, vamos analisar o caso $\mathcal{F}_{4}$, ou seja, apresentaremos condições nos campos iniciais para que a solução de $X_{\lambda}$, que tangencia o eixo- $x$, retorne a esse eixo perpendicularmente.

Vamos achar a solução do campo $X_{\lambda}(x, y)=(a x+b y+e \lambda, c x+d y+f \lambda)$ com condições iniciais $x(0)=p_{1}$ e $y(0)=0$. Para simplificar as fórmulas denotaremos $\Delta=\sqrt{-(a-d)^{2}-4 b c}$, que é um valor real pois por hipótese temos que $(a-d)^{2}+4 b c<0$. Um modo de se obter a solução do campo $X_{\lambda}$ é usar o método da eliminação para equações diferenciais ordinárias, ou seja, da segunda componente do campo temos que

$$
x=\frac{y^{\prime}-d y-f \lambda}{c}
$$


derivando e substituindo essa fração na primeira componente do campo $X_{\lambda}$ chegamos a seguinte equação

$$
y^{\prime \prime}-(a+d) y^{\prime}+(a d-b c) y=(e c-a f) \lambda .
$$

Estamos supondo que $c<0, a+d>0, a d-b c>0$ e $(a-d)^{2}+4 b c<0$. Resolvendo a equação diferencial de segunda ordem acima e substituindo a solução encontrada na igualdade que dá $x(t)$ obtemos, finalmente, a seguinte solução para o campo $X_{\lambda}$ com relação às condições iniciais $x(0)=p_{1}$ e $y(0)=0$,

$$
\begin{aligned}
x(t) & =\exp \left(\frac{a+d}{2} t\right) \frac{\lambda(e c-a f)}{c(b c-a d)}\left[-d \cos \left(\frac{\Delta t}{2}\right)+\frac{d^{2}-a d+2 b c}{\Delta} \operatorname{sen}\left(\frac{\Delta t}{2}\right)\right] \\
& +\frac{\lambda(d e-b f)}{b c-a d} \\
y(t) & =\exp \left(\frac{a+d}{2} t\right) \frac{e c-a f}{b c-a d} \lambda\left[\cos \left(\frac{\Delta t}{2}\right)-\frac{a+d}{\Delta} \operatorname{sen}\left(\frac{\Delta t}{2}\right)\right]-\frac{e c-a f}{b c-a d} \lambda .
\end{aligned}
$$

Agora que temos a solução do campo $X_{\lambda}$ que sai do foco e tangencia o conjunto de descontinuidade, vamos verificar quando essa solução cai exatamente na sela do campo de Filippov, ou seja, vamos igualar as soluções $x(t)$ e $y(t)$ encontradas acima a $-e \lambda / a$ e 0 , respectivamente, para obtermos a condição que caracteriza o caso $\mathcal{F}_{4}$. Assim, temos as seguintes igualdades

$$
\begin{aligned}
& \exp \left(\frac{a+d}{2} t\right)\left[-d \cos \left(\frac{\Delta t}{2}\right)+\frac{d^{2}-a d+2 b c}{\Delta} \operatorname{sen}\left(\frac{\Delta t}{2}\right)\right]=-\frac{b c}{a} \\
& \exp \left(\frac{a+d}{2} t\right)\left[\cos \left(\frac{\Delta t}{2}\right)-\frac{a+d}{\Delta} \operatorname{sen}\left(\frac{\Delta t}{2}\right)\right]=1
\end{aligned}
$$

Multiplicando a primeira igualdade de (3.7) por $-a / b c$, e igualando com a segunda igualdade obtemos

$$
\begin{aligned}
& \frac{a d}{b c} \cos \left(\frac{\Delta t}{2}\right)-\frac{a\left(d^{2}-a d+2 b c\right)}{b c \Delta} \operatorname{sen}\left(\frac{\Delta t}{2}\right)=\cos \left(\frac{\Delta t}{2}\right)-\frac{a+d}{\Delta} \operatorname{sen}\left(\frac{\Delta t}{2}\right) \\
\Rightarrow \quad & \operatorname{tg}\left(\frac{\Delta t}{2}\right)=\frac{\Delta}{d-a}
\end{aligned}
$$

para valores de $a$ distintos de $d$. Portanto, o valor de $t=t_{0}$ para o qual valem as duas igualdades de (3.7) é

$$
\frac{\Delta t_{0}}{2}=\operatorname{arctg}\left(\frac{\Delta}{d-a}\right) .
$$


A segunda igualdade de (3.7) pode ser reescrita como

$$
\frac{a+d}{2} t+\ln \left[\cos \left(\frac{\Delta t}{2}\right)-\frac{a+d}{\Delta} \operatorname{sen}\left(\frac{\Delta t}{2}\right)\right]=0 .
$$

Agora, notamos que se $h\left(t_{0}\right)$ é uma função tal que $\operatorname{tg}\left(h\left(t_{0}\right)\right)=t_{0}$, então $\operatorname{sen}\left(h\left(t_{0}\right)\right)=$ $t_{0} \cos \left(h\left(t_{0}\right)\right)$. Substituindo (3.8) e a observação acima em (3.9) obtemos

$$
\begin{aligned}
\frac{a+d}{2} \frac{2}{\Delta} \operatorname{arctg}\left(\frac{\Delta}{d-a}\right)+\ln & {\left[\cos \left(\operatorname{arctg}\left(\frac{\Delta}{d-a}\right)\right)\right.} \\
& \left.-\frac{a+d}{\Delta} \frac{\Delta}{d-a} \cos \left(\operatorname{arctg}\left(\frac{\Delta}{d-a}\right)\right)\right]=0 .
\end{aligned}
$$

Agora, como $\cos (\operatorname{arctg}(x))=\left(1+x^{2}\right)^{-1 / 2}$, chegamos finalmente na definição da função $G: \mathbb{R}^{4} \rightarrow \mathbb{R}$ dada por

$$
G(a, b, c, d)=\frac{a+d}{\Delta} \operatorname{arctg}\left(\frac{\Delta}{d-a}\right)+\ln \left(\frac{a}{\sqrt{-b c}}\right) .
$$

Em [F], e também em [KGR], uma expressão análoga para $G$ foi apresentada, e a diferença crucial na obtenção da nossa expressão para $G$ e a de Filippov é que em $[\mathrm{F}]$, e em [KGR], o conjunto de descontinuidade é a reta $y=1$.

Como consequência do resultado acima, temos que se $Z_{\lambda}$ é uma família de campos vetoriais descontínua que seja do tipo $\mathcal{F}_{1}, \mathcal{F}_{2}$ ou $\mathcal{F}_{4}$ então $\alpha$ pode ser positivo ou negativo, desde que seja satisfeita a desigualdade $c \alpha<a \beta$. Por outro lado, se $Z_{\lambda}$ é do tipo $\mathcal{F}_{3}$ então $\alpha$ também pode ser positivo ou negativo, desde que a desigualdade $c \alpha>a \beta$ seja satisfeita. Para qualquer um dos subcasos de foco no bordo, iremos considerar que $\alpha>0$.

Obs.: Se um campo vetorial é do tipo $\mathcal{F}_{1}, \mathcal{F}_{2}$ ou $\mathcal{F}_{4}$ então temos que $a>0$. Além disso, sabemos que $c<0$ e ce $-a f<0$, o que nos leva a considerar que $e$ e $f$ não podem ser simultaneamente negativos ou nulos pois, caso contrário, a desigualdade ce $-a f<0$ não seria satisfeita. Portanto, se $f$ é negativo então e é obrigatoriamente positivo, e se $f$ é positivo então $e$ pode ser positivo, negativo ou nulo, e se $f$ é nulo então $e$ é positivo.

\subsection{Bifurcações de $\mathcal{F}_{1}$ e $\mathcal{F}_{2}$ via regularização}

Nesta seção iremos analisar as bifurcações que ocorrem nas regularizações de campos vetoriais descontínuos que sejam do tipo $\mathcal{F}_{1}$ ou $\mathcal{F}_{2}$. Portanto, vamos supor que $a, b, c$, e $d$ 
não anulam a função $G(a, b, c, d)$.

A família de campos regularizados será definida no plano de coordenadas $\bar{x}, \bar{y}$ e de acordo com a seguinte função de transição

$$
\varphi_{\varepsilon}(\bar{y})=\frac{1}{2}+\frac{\bar{y}}{2 \sqrt{(\bar{y})^{2}+\varepsilon^{2}}},
$$

onde $\varepsilon>0$. Assim, se $Z_{\bar{\lambda}}=\left(X_{\bar{\lambda}}, Y_{\bar{\lambda}}\right)$ é uma FCD, a FCR é dada por

$$
Z_{\bar{\lambda}, \varepsilon}(\bar{x}, \bar{y})=\left(1-\varphi_{\varepsilon}(\bar{y})\right) Y_{\bar{\lambda}}(\bar{x}, \bar{y})+\varphi_{\varepsilon}(\bar{y}) X_{\bar{\lambda}}(\bar{x}, \bar{y}),
$$

onde estamos supondo que a função que define o conjunto $D$, da descontinuidade, é a projeção da segunda coordenada. Portanto, se $Z_{\bar{\lambda}}=\left(X_{\bar{\lambda}}, Y_{\bar{\lambda}}\right)$ onde

$$
\begin{aligned}
X_{\bar{\lambda}}(\bar{x}, \bar{y}) & =(a \bar{x}+b \bar{y}+e \bar{\lambda}, c \bar{x}+d \bar{y}+f \bar{\lambda}) \\
Y_{\bar{\lambda}}(\bar{x}, \bar{y}) & =(\bar{\alpha}, \bar{\beta})
\end{aligned}
$$

é uma FCD, a respectiva FCR é dada por

$$
\begin{gathered}
Z_{\bar{\lambda}, \varepsilon}(\bar{x}, \bar{y})=\left(\left(\frac{1}{2}-\frac{\bar{y}}{2 \sqrt{(\bar{y})^{2}+\varepsilon^{2}}}\right) \bar{\alpha}+\left(\frac{1}{2}+\frac{\bar{y}}{2 \sqrt{(\bar{y})^{2}+\varepsilon^{2}}}\right)(a \bar{x}+b \bar{y}+e \bar{\lambda}),\right. \\
\left.\left(\frac{1}{2}-\frac{\bar{y}}{2 \sqrt{(\bar{y})^{2}+\varepsilon^{2}}}\right) \bar{\beta}+\left(\frac{1}{2}+\frac{\bar{y}}{2 \sqrt{(\bar{y})^{2}+\varepsilon^{2}}}\right)(c \bar{x}+d \bar{y}+f \bar{\lambda})\right)
\end{gathered}
$$

que é uma família de campos vetoriais regulares a dois parâmetros.

Vamos fazer a seguinte mudança de variáveis e reescalonamento dos parâmetros na família de campos regularizados,

$$
\bar{x}=\varepsilon x, \quad \bar{y}=\varepsilon y, \quad \bar{\alpha}=\varepsilon \alpha, \quad \bar{\beta}=\varepsilon \beta, \quad \bar{\lambda}=\varepsilon \lambda .
$$

Este artifício é útil para desconsiderarmos o parâmetro $\varepsilon$ no cálculo das bifurcações da FCR, e logo após efetuado esses cálculos voltaremos às variáveis e parâmetros iniciais, interpretando os resultados obtidos.

Efetuando a mudança de variáveis e o reescalonamento dos parâmetros na FCR (3.11), 
que iremos de agora em diante denotar por $Z_{\lambda, R}$, obtemos

$$
\begin{gathered}
Z_{\lambda, R}(x, y)=\left(\left(\frac{1}{2}-\frac{y}{2 \sqrt{y^{2}+1}}\right) \alpha+\left(\frac{1}{2}+\frac{y}{2 \sqrt{y^{2}+1}}\right)(a x+b y+e \lambda),\right. \\
\left.\left(\frac{1}{2}-\frac{y}{2 \sqrt{y^{2}+1}}\right) \beta+\left(\frac{1}{2}+\frac{y}{2 \sqrt{y^{2}+1}}\right)(c x+d y+f \lambda)\right) .
\end{gathered}
$$

Como $c e-a f \neq 0$ as duas expressões seguintes, que dão os valores de $x$ e $\lambda$ das singularidades da FCR (3.12), estão bem definidas

$$
\begin{aligned}
& x=\frac{(\alpha f-\beta e)\left(1+2 y^{2}-2 y \sqrt{y^{2}+1}\right)+(b f-d e) y}{c e-a f} \\
& \lambda=\frac{(a \beta-\alpha c)\left(1+2 y^{2}-2 y \sqrt{y^{2}+1}\right)+(a d-b c) y}{c e-a f} .
\end{aligned}
$$

Seja $A=\left(a_{i j}\right), 1 \leq i, j \leq 2$, a matriz $D\left(Z_{\lambda, R}\right)(x, y)$, denominada matriz Jacobiana, cujos elementos são

$$
\begin{aligned}
& a_{11}=\left(\frac{1}{2}+\frac{y}{2 \sqrt{y^{2}+1}}\right) a \\
& a_{12}=\frac{-\alpha+a x+b y^{3}+2 b y+e \lambda+b\left(y^{2}+1\right)^{3 / 2}}{2\left(y^{2}+1\right)^{3 / 2}} \\
& a_{21}=\left(\frac{1}{2}+\frac{y}{2 \sqrt{y^{2}+1}}\right) c \\
& a_{22}=\frac{-\beta+c x+d y^{3}+2 d y+f \lambda+d\left(y^{2}+1\right)^{3 / 2}}{2\left(y^{2}+1\right)^{3 / 2}} .
\end{aligned}
$$

O determinante da matriz Jacobiana, ou simplesmente Jacobiano, da FCR (3.12) é dado por

$$
\operatorname{det}(A)=\frac{\sqrt{y^{2}+1}+y}{4\left(y^{2}+1\right)^{2}}\left((a d-b c)\left(y^{3}+2 y+\left(y^{2}+1\right)^{3 / 2}\right)+(a f-e c) \lambda-\beta a+\alpha c\right)
$$

ao passo que o traço da matriz Jacobiana vale

$$
\operatorname{tr}(A)=\frac{(a+d)\left(y^{3}+\left(y^{2}+1\right)^{3 / 2}\right)+(a+2 d) y+f \lambda-\beta+c x}{2\left(y^{2}+1\right)^{3 / 2}} .
$$


Para os próximos resultados vamos considerar no plano $(y, \lambda)$ a curva $\lambda(y)$ dada pela segunda equação de (3.13), também analisaremos as curvas $\operatorname{det}_{s}(A)(y)$ e $\operatorname{tr}_{s}(A)(y)$ que são dadas pela substituição dos valores de $x$ e $\lambda$, dadas por (3.13), nas expressões do determinante e do traço de $A$, dadas por (3.14) e (3.15), respectivamente. Assim, temos que

$$
\begin{aligned}
\operatorname{det}_{s}(A) & =\frac{y+\sqrt{y^{2}+1}}{4\left(y^{2}+1\right)^{2}}\left((a d-b c)\left(y+y^{3}+\left(1+y^{2}\right)^{3 / 2}\right)-2(\beta a-\alpha c)\left(1+y^{2}\right.\right. \\
& \left.\left.-y \sqrt{1+y^{2}}\right)\right) \\
\operatorname{tr}_{s}(A)= & \frac{1}{2\left(1+y^{2}\right)^{3 / 2}}\left((a+d)\left(1+y^{2}\right)\left(y+\sqrt{1+y^{2}}\right)-2 \beta\left(1+y^{2}-y \sqrt{1+y^{2}}\right)\right) .
\end{aligned}
$$

Lema 12. Seja $Z_{\lambda}=\left(X_{\lambda}, Y_{\lambda}\right)$ uma familia a 1-parâmetro, $\lambda$, de campos vetoriais descontínuos dada por (3.1) que pertença ao caso $\mathcal{F}_{1}$ ou $\mathcal{F}_{2}$. Então, a respectiva família de campos regularizados, $Z_{\lambda, R}$, dada por (3.12), possui valores do parâmetro onde ocorrem duas, uma ou nenhuma singularidade.

Demonstração. Seja $Z_{\lambda}$ uma FCD do tipo $\mathcal{F}_{1}$ ou $\mathcal{F}_{2}$. Vamos considerar a expressão de $\lambda$, dada por (3.13). Para simplificar as contas vamos denotar $a d-b c=n>0$, ce-af $=m<0$, $a \beta-\alpha c=l>0$, essa última desigualdade caracteriza os casos $\mathcal{F}_{1}$ e $\mathcal{F}_{2}$, conforme o lema 11. Considerando $\lambda$, dada por (3.13), em função de $y$ e substituindo as notações acima obtemos

$$
\lambda(y)=\frac{l\left(1+2 y^{2}-2 y \sqrt{y^{2}+1}\right)+n y}{m} .
$$

Das hipóteses iniciais sobre $l, m$ e $n$ os limites quando $y$ vai para $+\infty$ ou $-\infty$ de $\lambda(y)$ são ambos $-\infty$. Além disso, essa curva possui um único ponto crítico dado por $y=y^{*}$ onde

$$
\begin{aligned}
y^{*} & =\frac{\left(\left(-576 n^{2} l^{2}+3456 l^{4}-n^{4}+24\left(n^{2}+8 l^{2}\right) \sqrt{324 l^{2}+3 n^{2}} l\right) n^{2}\right)^{1 / 3}}{24 l n} \\
& -\frac{\left(192 l^{2}-n^{2}\right) n}{24 l\left(\left(-576 n^{2} l^{2}+3456 l^{4}-n^{4}+24\left(n^{2}+8 l^{2}\right) \sqrt{324 l^{2}+3 n^{2}} l\right) n^{2}\right)^{1 / 3}}-\frac{n}{24 l}
\end{aligned}
$$

definido para valores de $l$ distintos de $\sqrt{3} n / 24$. 
A segunda derivada de $\lambda(y)$ em função de $y$ é dada por

$$
\lambda^{\prime \prime}(y)=\frac{4 l}{m}-\frac{2 l y\left(3+2 y^{2}\right)}{\left(1+y^{2}\right)^{3 / 2} m}
$$

que nunca se anula, tende a zero, por valores negativos, quando $y$ vai a infinito, e tende a $8 l / m<0$ quando $y$ vai a $-\infty$. Assim, temos que $\lambda^{\prime \prime}(y)<0$ para qualquer valor de $y$, implicando que $\lambda^{\prime}(y)$ é uma função estritamente decrescente. Além disso, o fato $\lambda^{\prime \prime}(y)<0$ implica que $\lambda(y)$ é uma função côncava, e $y^{*}$ um ponto de máximo.

Quando $l=\sqrt{3} n / 24$ a função $\lambda(y)$ ainda tem um único ponto crítico dado por

$$
y=-\frac{\sqrt{3}}{3}\left(1+\frac{5 \sqrt[3]{4}}{4}\right) \approx-1,722958
$$

Além disso, a segunda derivada de $\lambda(y)$ nunca se anula e é negativa para qualquer valor de $y$. Portanto, $\lambda^{\prime}(y)$ é estritamente decrescente e $\lambda(y)$ é côncava.

Por essas observações, concluímos que existe um valor crítico do parâmetro dado por $\lambda=\lambda^{*}$, definido pela substituição de $y$ por $y^{*}$ na expressão de $\lambda$ dada por (3.13).

Lema 13. Seja $Z_{\lambda}=\left(X_{\lambda}, Y_{\lambda}\right)$ uma $F C D$ a 1-parâmetro dada por (3.1) que pertença aos casos $\mathcal{F}_{1}$ ou $\mathcal{F}_{2}$. Então existe um único $y_{\Delta}$ com $y^{*}<y_{\Delta}$, onde $y^{*}$ é dado por (3.18), tal que

a) para valores de $y$ estritamente menores que $y^{*}$ as singularidades são selas, em $y^{*}$ o Jacobiano da FCR, dado por (3.16), se anula e é positivo quando $y>y^{*}$

b) em $y_{\Delta}$ a função $\Delta(y)=\left(\operatorname{tr}_{s}(A)(y)\right)^{2}-4 \operatorname{det}_{s}(A)(y)$ se anula, é positiva para valores de y menores que $y_{\Delta}$ e negativa caso contrário.

Demonstração. Substituindo o valor de $\lambda$ dado por (3.13) em (3.14) obtemos que o determinante se anula exatamente no ponto crítico $y^{*}$ do lema anterior, que já vimos ser único.

O limite do determinante, dado por (3.16), quando $y$ vai a infinito vale $a d-b c>0$, e quando $y$ tende a $-\infty$ o determinante tende a zero. Além disso, a derivada do determinante no ponto $y^{*}$, é positiva. Logo, como $y^{*}$ é a única raiz do determinante, se $y>y^{*}$ o determinante é positivo e será negativo se $y<y^{*}$. Provando assim o item a).

Calculando o discriminante do polinômio característico da matriz $A$ aplicado na curva 
de singularidades, temos

$$
\begin{aligned}
\Delta_{s}(y) & =\left((a-d)^{2}+4 b c\right) \frac{\left(y+\sqrt{1+y^{2}}\right)^{2}}{4\left(1+y^{2}\right)}+\beta^{2} \frac{\left(1+y^{2}-y \sqrt{1+y^{2}}\right)^{2}}{\left(1+y^{2}\right)^{3}} \\
& +((a-d) \beta-2 c \alpha)\left(1+y^{2}-y \sqrt{1+y^{2}}\right) \frac{y+\sqrt{1+y^{2}}}{\left(1+y^{2}\right)^{2}}
\end{aligned}
$$

Podemos reescrever (3.19) do seguinte modo

$$
\Delta_{s}(y)=\frac{C_{1} \xi^{2}+C_{2} \zeta \xi+C_{3} \zeta^{2}}{1+y^{2}}
$$

onde

$$
\begin{aligned}
C_{1} & =\frac{(a-d)^{2}+4 b c}{4} \\
C_{2} & =(a-d) \beta-2 c \alpha \\
C_{3} & =\beta^{2} \\
\xi & =y+\sqrt{1+y^{2}} \\
\zeta & =\frac{1+y^{2}-y \sqrt{1+y^{2}}}{1+y^{2}} .
\end{aligned}
$$

Como $\xi \neq 0$, chamamos $\omega=\frac{\zeta}{\xi}$ para finalmente obtermos

$$
\Delta_{s}(y)=\frac{\xi^{2}}{1+y^{2}}\left(C_{3} \omega^{2}+C_{2} \omega+C_{1}\right) .
$$

Portanto, $\Delta_{s}(y)=0$ se

$$
\omega=\frac{-C_{2} \pm \sqrt{C_{2}^{2}-4 C_{1} C_{3}}}{2 C_{3}} .
$$

Mas,

$$
\omega=\frac{1+y^{2}-y \sqrt{1+y^{2}}}{\left(1+y^{2}\right)\left(y+\sqrt{1+y^{2}}\right)}
$$

que é estritamente positivo para qualquer valor de $y$. Como $C_{1}<0$ e $C_{3}>0$ as raízes de (3.21) têm sinais opostos. Portanto, apenas uma das raízes nos dará o ponto $y_{\Delta}$ vamos 
denotar de $r$ essa raiz. Assim, o ponto $y_{\Delta}$ é dado pela igualdade

$$
\frac{1+y^{2}-y \sqrt{1+y^{2}}}{\left(1+y^{2}\right)\left(y+\sqrt{1+y^{2}}\right)}=r
$$

manipulando essa igualdade chegamos a

$$
4 r y^{3}+r^{2} y^{2}+4 r y+r^{2}-1=0
$$

que tem como solução

$$
y_{\Delta}=\frac{W}{12 r}-\left(4-\frac{r^{2}}{12}\right) \frac{r}{W}-\frac{r}{12}
$$

onde

$$
W=\left[\left(-r^{4}-144 r^{2}+216+12\left(r^{2}+2\right) \sqrt{ } 3\left(r^{2}+27\right)\right) r^{2}\right]^{1 / 3} .
$$

A próxima figura apresenta o esquema gráfico dos dois lemas acima.

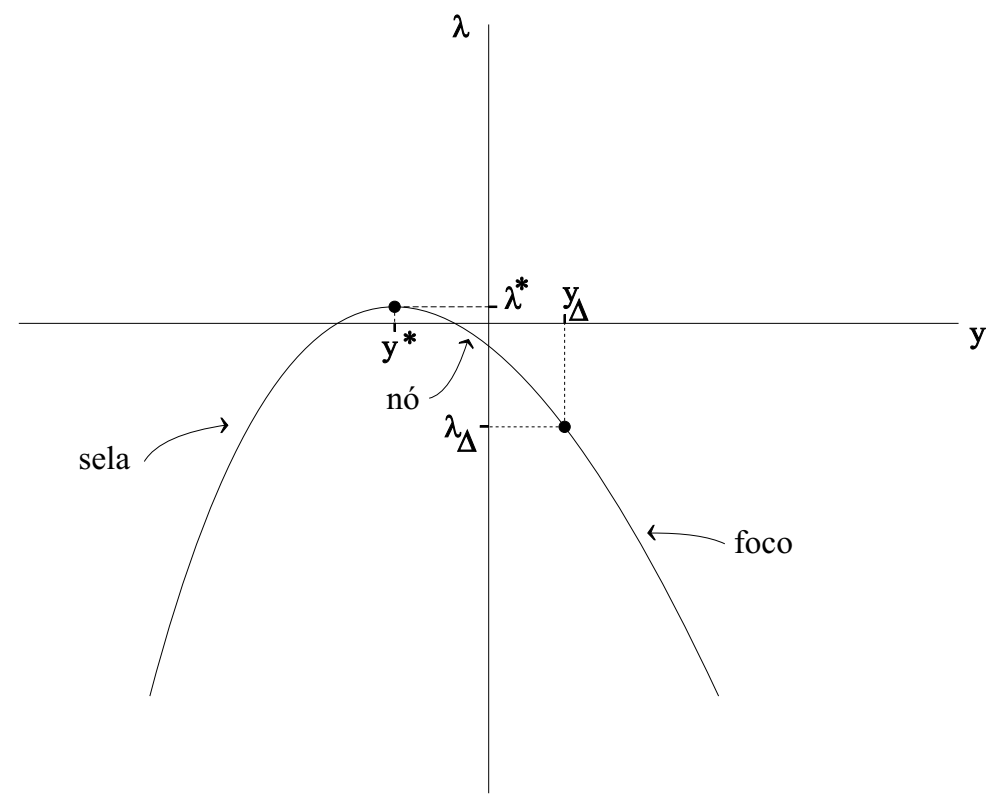

figura 8: Gráfico da curva $\lambda(y)$

Os dois lemas anteriores nos dão elementos para o próximo resultado que exibe o cálculo da curva de sela-nó para uma FCR, nas variáveis e parâmetros iniciais, cuja respectiva FCD pertença aos casos $\mathcal{F}_{1}$ ou $\mathcal{F}_{2}$. 
Agora vamos apresentar a bifurcação que ocorre no segundo caso, essa bifurcação sozinha não explica completamente o que ocorre $\operatorname{com} \mathcal{F}_{1}$ quando variamos o parâmetro. Para isso, iremos utilizar o seguinte teorema devido a Sotomayor veja $[\mathrm{GH}]$, [P], e principalmente [S4]. Vamos enunciá-lo para o caso onde o espaço ambiente é o $\mathbb{R}^{2}$, conforme consta em $[\mathrm{GH}]$.

Teorema 14. Seja $\dot{x}=F(x, \lambda)$ uma família a um parâmetro de equações diferenciais em $\mathbb{R}^{2}$ dependendo do parâmetro real $\lambda$. Quando $\lambda=\lambda_{0}$, assuma que exista uma singularidade $p_{0}=\left(x_{0}, y_{0}\right)$ que satisfaça as seguintes hipóteses:

(SN1) a matriz Jacobiana de $F$ em $\left(p_{0}, \lambda_{0}\right)$ tem um único autovalor nulo $\mu_{0}$ com autovetores $v$ e $w$, à direita e à esquerda, respectivamente.

(SN2) vale a seguinte desigualdade

$$
\left\langle w, \frac{d}{d \lambda} F\left(p_{0}, \lambda_{0}\right)\right\rangle \neq 0
$$

onde $<,>$ denota o produto escalar usual do plano

(SN3) vale a seguinte desigualdade

$$
\left\langle w, D_{x}^{2} F\left(p_{0}, \lambda_{0}\right)(v, v)\right\rangle \neq 0 .
$$

Então existe uma curva suave de singularidades em $\mathbb{R}^{2} \times \mathbb{R}$ passando por $\left(p_{0}, \lambda_{0}\right)$, tangente ao hiperplano $\mathbb{R}^{2} \times\left\{\lambda_{0}\right\}$. Dependendo dos sinais das expressões em (SN2) e (SN3), não existem singularidades próximas a $\left(p_{0}, \lambda_{0}\right)$ quando $\lambda<\lambda_{0}\left(\lambda>\lambda_{0}\right)$ e duas singularidades próximas a $\left(p_{0}, \lambda_{0}\right)$ para cada valor do parâmetro $\lambda>\lambda_{0}\left(\lambda<\lambda_{0}\right)$. As duas singularidades para $\dot{x}=F(x, \lambda)$ próximas a $\left(p_{0}, \lambda_{0}\right)$ são hiperbólicas.

Proposição 15. Seja $Z_{\lambda}=\left(X_{\lambda}, Y_{\lambda}\right)$ uma $F C D$ dada por (3.1) que pertence ao caso $\mathcal{F}_{1}$ ou $\mathcal{F}_{2}$. Então a $F C R, Z_{\lambda, R}$, admite uma bifurcação do tipo sela-nó.

Demonstração. Seja a FCR, $Z_{\lambda}=\left(X_{\lambda}, Y_{\lambda}\right)$, dada por (3.1). Como a matriz Jacobiana da FCR é uma matriz real, $A$, de ordem 2, para que um dos seus autovalores seja nulo devemos ter que o Jacobiano deve ser nulo. Portanto, resolvendo $\operatorname{det}_{s}(A)=0$, onde $\operatorname{det}_{s}$ é dada por (3.16), teremos o ponto $p^{*}=\left(x^{*}, y^{*}\right)$ e o parâmetro $\lambda^{*}$ que irão satisfazer (SN1).

Uma outra forma de expressar $y^{*}$, dada pelo Lema 12, é através da seguinte expressão, onde $n=a d-b c$ e $l=a \beta-c \alpha$,

$$
y^{*}=\frac{\xi(2 l-n \xi)}{n \xi+2 l}
$$


onde

$$
\begin{aligned}
& \xi=\frac{\left(\left(144 l^{2} n^{2}+3456 l^{4}+n^{4}+192 \sqrt{3 n^{2}+324 l^{2}} l^{3}\right) n^{2}\right)^{1 / 3}}{24 l n}+\frac{n}{24 l} \\
& +\frac{\left(96 l^{2}+n^{2}\right) n}{24 l\left(\left(144 l^{2} n^{2}+3456 l^{4}+n^{4}+192 \sqrt{3 n^{2}+324 l^{2}} l^{3}\right) n^{2}\right)^{1 / 3}} .
\end{aligned}
$$

Agora que já temos o valor de $y^{*}$ basta substituí-lo em $x_{s}$ e $\lambda_{s}$, dados por (3.13), donde obtemos $p^{*}=\left(x^{*}, y^{*}\right)$ e $\lambda^{*}$ que é a singularidade onde a matriz Jacobiana da FCR, no parâmetro $\lambda^{*}$, tem um autovalor $\mu_{1}$ nulo. Para finalizar (SN1) vamos apresentar os autovetores à direita e à esquerda do autovalor nulo.

Um autovetor à direita é o vetor não-nulo $v=\left(v_{1}, v_{2}\right)$ que satisfaz $A\left(p^{*}, \lambda^{*}\right) v=\mu_{1} v=0$, pois queremos associá-lo ao autovalor $\mu_{1}=0$. Assim,

$$
v=\left(1, \frac{-a\left(\sqrt{1+\left(y^{*}\right)^{2}}+y^{*}\right)\left(1+\left(y^{*}\right)^{2}\right)}{-\alpha+a x^{*}+b\left(y^{*}\right)^{3}+2 b y^{*}+e \lambda^{*}+b\left(1+\left(y^{*}\right)^{2}\right)^{3 / 2}}\right)
$$

analogamente, o autovetor à esquerda, associado ao autovalor $\mu_{1}=0$, é a solução não-nula de $w^{T} A\left(p^{*}, \lambda^{*}\right)=0$ que é dado por

$$
w=\left(1,-\frac{a}{c}\right)
$$

Isso finaliza a verificação da hipótese (SN1) do Teorema 14, agora vamos às outras hipóteses.

Temos que verificar que o seguinte produto escalar é não-nulo

$$
p_{1}=\left\langle w, \frac{d}{d \lambda} F\left(p^{*}, \lambda^{*}\right)\right\rangle,
$$

onde

$$
F(x, y, \lambda)=Z_{\lambda, \varepsilon}(x, y, \lambda)=\left(Z_{\lambda, \varepsilon}^{1}(x, y), Z_{\lambda, \varepsilon}^{2}(x, y)\right)
$$

Para isso, temos que

$$
\frac{d}{d \lambda} Z_{\lambda, \varepsilon}\left(x^{*}, y^{*}, \lambda^{*}\right)=\left(\left(\frac{1}{2}+\frac{y^{*}}{2 \sqrt{\left(y^{*}\right)^{2}+1}}\right) e,\left(\frac{1}{2}+\frac{y^{*}}{2 \sqrt{\left(y^{*}\right)^{2}+1}}\right) f\right)
$$


multiplicando escalarmente por $w$ resulta em

$$
p_{1}=\left(\frac{1}{2}+\frac{y^{*}}{2 \sqrt{\left(y^{*}\right)^{2}+1}}\right)\left(\frac{c e-a f}{c}\right) .
$$

Como temos de antemão que $c e-a f<0$, a expressão (3.24) se anulará caso $y^{*}+$ $\sqrt{1+\left(y^{*}\right)^{2}}=0$, mas a função $y^{*}+\sqrt{1+\left(y^{*}\right)^{2}}$ não se anula e é sempre positiva para qualquer valor de $y^{*}$. Portanto, $p_{1} \neq 0$ o que prova (SN2).

Agora, vamos verificar que o seguinte produto escalar é não-nulo

$$
p_{2}=\left\langle w, D_{x}^{2} Z_{\lambda, \varepsilon}\left(p^{*}, \lambda^{*}\right)(v, v)\right\rangle .
$$

Para isso, precisaremos calcular $D_{x}^{2} Z_{\lambda, \varepsilon}\left(p^{*}, \lambda^{*}\right)(v, v)$, e adotaremos a seguinte notação $(x, y)=$ $\left(x_{1}, x_{2}\right)$. Assim,

$$
D_{x}^{2} Z_{\lambda, \varepsilon}\left(p^{*}, \lambda^{*}\right)(v, v)=\left(\sum_{i, j=1}^{2} \frac{\partial^{2} Z_{\lambda, \varepsilon}^{1}}{\partial x_{i} \partial x_{j}}\left(p^{*}, \lambda^{*}\right) v_{j} v_{i}, \sum_{i, j=1}^{2} \frac{\partial^{2} Z_{\lambda, \varepsilon}^{2}}{\partial x_{i} \partial x_{j}}\left(p^{*}, \lambda^{*}\right) v_{j} v_{i}\right)
$$

Logo, a primeira componente da segunda derivada em relação a $x \in \mathbb{R}^{2}$ da FCR é

$$
\begin{aligned}
& \frac{\partial^{2} Z_{\lambda, \varepsilon}^{1}}{\partial x_{1}^{2}}\left(p^{*}, \lambda^{*}\right) v_{1}^{2}+2 \frac{\partial^{2} Z_{\lambda, \varepsilon}^{1}}{\partial x_{1} \partial x_{2}}\left(p^{*}, \lambda^{*}\right) v_{1} v_{2}+\frac{\partial^{2} Z_{\lambda, \varepsilon}^{1}}{\partial x_{2}^{2}}\left(p^{*}, \lambda^{*}\right) v_{2}^{2} \\
& =\frac{a v_{1} v_{2}}{\left(\left(y^{*}\right)^{2}+1\right)^{3 / 2}}+\frac{y^{*}\left(3 \alpha-3 a x^{*}-3 e \lambda^{*}-b y^{*}\right)+2 b}{2\left(\left(y^{*}\right)^{2}+1\right)^{5 / 2}} v_{2}^{2}
\end{aligned}
$$

a segunda componente da derivada é

$$
\begin{aligned}
& \frac{\partial^{2} Z_{2}}{\partial x_{1}^{2}}\left(p^{*}, \lambda^{*}\right) v_{1}^{2}+2 \frac{\partial^{2} Z_{2}}{\partial x_{1} \partial x_{2}}\left(p^{*}, \lambda^{*}\right) v_{1} v_{2}+\frac{\partial^{2} Z_{2}}{\partial x_{2}^{2}}\left(p^{*}, \lambda^{*}\right) v_{2}^{2} \\
& =\frac{c v_{1} v_{2}}{\left(\left(y^{*}\right)^{2}+1\right)^{3 / 2}}+\frac{y^{*}\left(3 \beta-3 c x^{*}-d y^{*}-3 f \lambda^{*}\right)+2 d}{2\left(\left(y^{*}\right)^{2}+1\right)^{5 / 2}} v_{2}^{2}
\end{aligned}
$$

Multiplicando escalarmente essas componentes pelo vetor $w$ obtemos

$$
p_{2}=\frac{v_{2}^{2}\left(n\left(y^{*}\right)^{2}-3 l y^{*}-3(c e-a f) y^{*} \lambda^{*}-2 n\right)}{2 c\left(\left(y^{*}\right)^{2}+1\right)^{5 / 2}} .
$$


Notamos que se $m=c e-a f$ então

$$
\begin{aligned}
& b f-d e=\frac{-b m-e n}{a} \\
& f \alpha-e \beta=\frac{f l-\beta m}{c} .
\end{aligned}
$$

Substituindo esses valores na expressão de $x^{*}$, dada por (3.13), temos que

$$
x^{*}=\frac{a(f l-\beta m)\left(1+2\left(y^{*}\right)^{2}-2 y^{*} \sqrt{1+\left(y^{*}\right)^{2}}\right)-c(b m+e n) y^{*}}{a c m} \text {. }
$$

Substituindo essa nova expressão de $x^{*}$, a expressão para $\lambda^{*}$ e o valor encontrado de $v_{2}$ em $p_{2}$, dada por (3.26), obtemos

$$
\begin{aligned}
& p_{2}=\left[a^{2} c m^{2}\left(y^{*}+\left(y^{*}\right)^{3}+\left(1+\left(y^{*}\right)^{2}\right)^{3 / 2}\right)^{2}\left(-\left(3+l y^{*}\right)\left(1+\left(y^{*}\right)^{2}\right)+3 l\left(y^{*}\right)^{2} \sqrt{1+\left(y^{*}\right)^{2}}\right)\right] \\
& \quad\left[\left(-(a \beta+c \alpha) m+a f l\left(1+2\left(y^{*}\right)^{2}-2 y^{*} \sqrt{1+\left(y^{*}\right)^{2}}\right)+2 a \beta m y^{*}\left(-y^{*}+\sqrt{1+\left(y^{*}\right)^{2}}\right)\right.\right. \\
& \left.\left.\quad+b c m y^{*}\left(1+\left(y^{*}\right)^{2}\right)+\operatorname{cel}\left(1+2\left(y^{*}\right)^{2}-2 y^{*} \sqrt{1+\left(y^{*}\right)^{2}}\right)+b c m\left(1+\left(y^{*}\right)^{2}\right)^{3 / 2}\right)^{2}\left(1+\left(y^{*}\right)^{2}\right)^{5 / 2}\right]^{-1} .
\end{aligned}
$$

Por hipótese temos que $a, c$ e $m=c e-a f$ são todos não nulos. Além disso, $\left(y^{*}+\left(y^{*}\right)^{3}+\right.$ $\left.\left(1+\left(y^{*}\right)^{2}\right)^{3 / 2}\right)^{2} \neq 0$ para qualquer valor de $y^{*}$. O último termo de $p_{2}$, a saber, $-\left(3+l y^{*}\right)(1+$ $\left.\left(y^{*}\right)^{2}\right)+3 l\left(y^{*}\right)^{2} \sqrt{1+\left(y^{*}\right)^{2}}$ se anula em pontos distintos de $y^{*}$. Portanto, $p_{2} \neq 0$ provando o item (SN3) e a proposição.

Obs.: Seja $Z_{\lambda}$ uma FCD que pertença ao caso $\mathcal{F}_{1}$ ou $\mathcal{F}_{2}$. A curva no plano $(\varepsilon, \bar{\lambda})$ que determina as selas-nó para a FCR, nas variáveis e parâmetros originais, é uma reta.

De fato, o ponto sela-nó, pela Proposição anterior, ocorrerá para a FCR da hipótese quando o parâmetro $\lambda$ for igual a

$$
\lambda^{*}=\frac{l\left(1+2 \xi^{2}-2 \xi \sqrt{\xi^{2}+1}\right)+n \xi}{m}
$$


onde

$$
\begin{aligned}
& \xi=\frac{\left(\left(-576 n^{2} l^{2}+3456 l^{4}-n^{4}+24\left(n^{2}+8 l^{2}\right) \sqrt{324 l^{2}+3 n^{2}} l\right) n^{2}\right)^{1 / 3}}{24 l n} \\
& -\frac{\left(192 l^{2}-n^{2}\right) n}{24 l\left(\left(-576 n^{2} l^{2}+3456 l^{4}-n^{4}+24\left(n^{2}+8 l^{2}\right) \sqrt{324 l^{2}+3 n^{2}} l\right) n^{2}\right)^{1 / 3}}-\frac{n}{24 l},
\end{aligned}
$$

$l=a \beta-c \alpha, m=c e-a f$ e $n=a d-b c$.

Notamos que para quaisquer valores de $l$ e $m$ o ponto $\lambda^{*}$ tende a zero quando $n$ tende a zero. Além disso, para qualquer valor de $m$ existe uma única raiz de $\lambda^{*}$ em $n_{0}=-l(1+$ $\left.2 \xi^{2}-2 \xi \sqrt{1+\xi^{2}}\right) \xi^{-1}$ que se desloca para a direita à medida que aumentamos $l$. A função $\lambda^{*}$ é negativa para valores de $n$ menores que $n_{0}$ e é positiva se $n_{0}<n<8 \sqrt{3} l$.

Portanto, voltando às variáveis e parâmetros originais temos que a curva sela-nó é dada por uma reta pois $\lambda=\varepsilon \bar{\lambda}$. Assim, a reta sela-nó tem inclinação dada por $\lambda^{*}$ que pode ser positivo, negativo ou nulo, dependendo do valor de $n$ com relação a $n_{0}$.

Obs.: O traço da matriz Jacobiana da FCR calculado no ponto sela-nó, ou seja, em $y^{*}$ dado por (3.18), é tal que fixado um valor para $a+d=u>0$ e $l$, o seu conjunto de definição é $n \in(0,8 \sqrt{3} l)$. O traço tende a $u$ para qualquer valor de $l$ quando $n$ tende a zero, e se anula em um único ponto dado por $n=l u$, à direita desse ponto o traço é negativo. Assim, para que o traço seja positivo devemos ter que $n<l u$, ou ainda, que $a d-b c<(a \beta-\alpha c)(a+d)$ implicando que a sela-nó será repulsora se

$$
\frac{a d-b c}{a \beta-\alpha c}<a+d
$$

e atratora caso contrário.

Ex.: Um exemplo de uma curva dos parâmetros das singularidades de uma FCR, e da curva sela-nó no plano $(\varepsilon, \lambda)$ é dado na figura 6 onde supomos que $\alpha=1, a=3, b=4,9$, $c=-2,6, d=3,5, e=2$, e $f=1$. Nesse exemplo temos que $l=5,6, m=-8,2 n=23,24 \mathrm{e}$ $u=6,5$. Além disso, $y^{*}=-0,416$, aproximadamente, e $\lambda^{*}=-0,3557$. Na figura 6 o gráfico à esquerda é a curva das singularidades da FCR e o gráfico da direita contém a curva de sela-nó. 

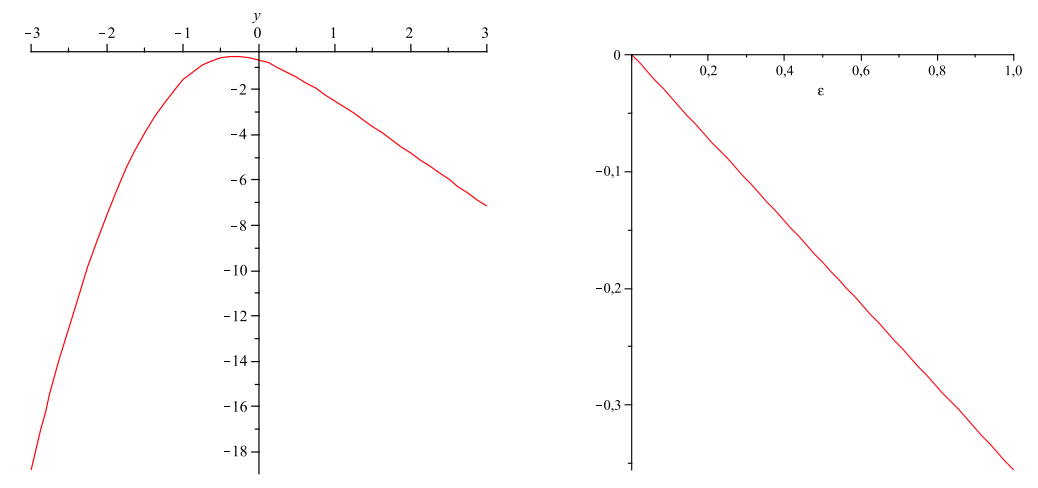

figura 9: Gráficos da curva $\lambda$ e da sela-nó

\subsection{Bifurcação de $\mathcal{F}_{1}$ via regularização - continuação}

O principal resultado desta seção apresenta as bifurcações que ocorrem em uma família de campos vetoriais regularizados associada a uma FCD que pertença ao caso $\mathcal{F}_{1}$. Dividimos o estudo desse caso em vários lemas que utilizaremos para provar o Teorema 19.

Os lemas da seção anterior provaram a existência da sela, da passagem do foco para nó e a existência de uma bifurcação do tipo sela-nó. O próximo lema trata da existência de um único ponto, $y_{h}$, onde o traço jacobiano se anula.

Lema 16. Seja $Z_{\lambda}=\left(X_{\lambda}, Y_{\lambda}\right)$ uma FCD dada por (3.1), que é do tipo $\mathcal{F}_{1}$ ou $\mathcal{F}_{2}$. Se a+ $d<\frac{(a d-b c) \beta}{a \beta-c \alpha}$, então existe um único valor $y_{h}$ que anula o traço da matriz Jacobiana da respectiva FCR, e é tal que $y_{\Delta}<y_{h}$, onde $y_{\Delta}$ é dado pelo Lema 13.

Demonstração. Substituindo os valores encontrados de $x$ e $\lambda$ dados por (3.13) em (3.15) obtemos que a expressão do traço na curva de singularidades é dada por

$$
\operatorname{tr}_{s}=\frac{u\left(y^{3}+y+y^{2} \sqrt{y^{2}+1}+\sqrt{y^{2}+1}\right)-2 \beta\left(y^{2}-\sqrt{y^{2}+1} y+1\right)}{2\left(y^{2}+1\right)^{3 / 2}}
$$

onde $u=a+d$.

Portanto, a única singularidade real, onde o traço da matriz Jacobiana de $Z_{\lambda, R}$ se anula tem ordenada dada por $^{1}$

$$
y_{h}=\frac{\Psi(u, \beta)^{2}-192 u^{2} \beta^{2}+u^{4}-u^{2} \Psi(u, \beta)}{24 u \beta \Psi(u, \beta)},
$$

\footnotetext{
${ }^{1} \mathrm{O}$ estudo das funções $\Psi$ e $y_{h}$ será dada adiante.
} 
onde $\Psi: \mathbb{R}^{2} \rightarrow \mathbb{R}$ é a seguinte função

$$
\Psi(u, \beta)=\left[\left(-u^{4}-576 u^{2} \beta^{2}+3456 \beta^{4}+24 \beta\left(u^{2}+8 \beta^{2}\right) \sqrt{3 u^{2}+324 \beta^{2}}\right) u^{2}\right]^{1 / 3} .
$$

A função $\Psi(u, \beta)$ se anula quando $u=0$ e $u= \pm 8 \sqrt{3} \beta$. Portanto, vamos supor que $u \neq 8 \sqrt{3} \beta$, uma vez que $u>0$ é uma das hipóteses iniciais. Assim, com essa hipótese adicional o valor $y_{h}$ está bem definido. Quando $u=8 \sqrt{3} \beta$ temos que $y_{h}$ tende a $-\frac{5 \sqrt[3]{4}}{4 \sqrt{3}}-\frac{1}{\sqrt{3}}$ que vale, aproximadamente, $-1,722953$.

Notamos que se $n=l u / \beta$, onde $n=a d-b c$ e $l=a \beta-c \alpha$, então o ponto $y_{h}$ coincide com o ponto $y^{*}$ onde o Jacobiano se anula. Porém tal caso não nos interessa no momento, de fato iremos tratar de tal ponto na seção que envolve o caso $\mathcal{F}_{4}$. Por outro lado, se $n>l u / \beta$ então temos que $y_{h}>y^{*}$. Além disso, temos que

$$
\Delta\left(y_{h}\right)=\left(\operatorname{tr}_{s}(A)\left(y_{h}\right)\right)^{2}-4 \operatorname{det}_{s}(A)\left(y_{h}\right)=-4 \operatorname{det}_{s}(A)\left(y_{h}\right),
$$

e como $y_{h}>y^{*}$, temos que o Jacobiano é positivo, implicando que $\Delta\left(y_{h}\right)<0$. Portanto, como $\Delta\left(y^{*}\right)>0$, o ponto $y_{\Delta}$, onde $\Delta(y)$ se anula, está em $\left(y^{*}, y_{h}\right)$. Agora, se $n<l u / \beta$ então $y_{h}<y^{*}$, e este caso também não nos interessa por apresentar apenas selas.

O próximo lema trata da bifurcação de Hopf, cujo ciclo limite resultante é atrator, uma vez que o primeiro número de Lyapunov é negativo.

O cálculo do primeiro número de Lyapunov envolve os coeficientes do polinômio de Taylor até a terceira ordem das componentes da FCR, $Z_{\lambda, R}=\left(Z_{\lambda, R}^{1}, Z_{\lambda, R}^{2}\right)$, calculado na curva de singularidades onde o traço da matriz Jacobiana se anula. Assim, temos que

$$
\begin{aligned}
& Z_{\lambda, R}^{1}(x, y)=A x+B y+Z_{1}^{1}(x, y)+Z_{2}^{1}(x, y)+R(x, y) \\
& Z_{\lambda, R}^{2}(x, y)=C x+D y+Z_{1}^{2}(x, y)+Z_{2}^{2}(x, y)+S(x, y)
\end{aligned}
$$

onde $R$ e $S$ são restos com termos de ordem maior ou igual a quatro. As componentes $A, B, C$ e $D$ são as derivadas parciais de $Z_{\lambda, R}^{1}$ e $Z_{\lambda, R}^{2}$ em relação a $x$ e $y$, respectivamente. 
No nosso caso, temos que $A=a, B=b, C=c$ e $D=d$. Os outros polinômios são dados por

$$
\begin{aligned}
& Z_{1}^{1}=A_{20} x^{2}+A_{11} x y+A_{02} y^{2} \\
& Z_{1}^{1}=A_{30} x^{3}+A_{21} x^{2} y+A_{12} x y^{2}+A_{03} y^{3} \\
& Z_{1}^{2}=B_{20} x^{2}+B_{11} x y+B_{02} y^{2} \\
& Z_{1}^{2}=B_{30} x^{3}+B_{21} x^{2} y+B_{12} x y^{2}+B_{03} y^{3}
\end{aligned}
$$

onde se $k=i+j$, para $i, j=0,1,2,3$,

$$
A_{i j}=\frac{\partial^{k} Z_{\lambda, R}^{1}}{k ! \partial x^{i} \partial y^{j}}
$$

a mesma fórmula é válida para os $B_{i j}$, onde basta substituir $Z_{\lambda, R}^{1}$ por $Z_{\lambda, R}^{2}$. Assim, o primeiro número de Lyapunov, $l_{1}$, é dado por

$$
\begin{aligned}
l_{1} & =-\frac{\pi}{4 b \delta^{3 / 2}}\left\{a c\left(A_{11}^{2}+A_{11} B_{02}+A_{02} B_{11}\right)+a b\left(B_{11}^{2}+A_{20} B_{11}+A_{11} B_{20}\right)\right. \\
& +c^{2}\left(A_{11} A_{02}+2 A_{02} B_{02}\right)-2 a c\left(B_{02}^{2}-A_{20} A_{02}\right)-2 a b\left(A_{20}^{2}-B_{20} B_{02}\right) \\
& -b^{2}\left(2 A_{20} B_{20}+B_{11} B_{20}\right)+\left(b c-2 a^{2}\right)\left(B_{11} B_{02}-A_{11} A_{20}\right)-\left(a^{2}+b c\right) \\
& {\left.\left[3\left(c B_{03}-b A_{30}\right)+2 a\left(A_{21}+B_{12}\right)+\left(c A_{12}-b B_{21}\right)\right]\right\} }
\end{aligned}
$$

onde $\delta$ é o Jacobiano, ou seja, $\delta=a d-b c$.

Obs.: A fórmula (3.32) foi obtida do livro [A], outros livros que possuem a mesma fórmula são $[\mathrm{P}]$ e $[\mathrm{K}]$.

Vimos no lema anterior que o ponto $y_{h}$ dado por (3.30) é o ponto onde o traço da matriz Jacobiana se anula. Ainda pelo lema anterior temos que $y_{h}>y^{*}$ donde concluímos, pelo lema 13, que o determinante nesse ponto é positivo. Além disso, vimos que $y_{h}$ depende da função $\Psi(u, \beta)$ dada por (3.31). Seja $\Phi: \mathbb{R}^{2} \rightarrow \mathbb{R}$ a função definida pelo lado direito de (3.30), ou seja,

$$
\Phi(u, \beta)=\frac{\Psi(u, \beta)^{2}-192 u^{2} \beta^{2}+u^{4}-u^{2} \Psi(u, \beta)}{24 u \beta \Psi(u, \beta)}
$$

onde $u=a+d$ e $\Psi(u, \beta)$ é dado por (3.31), então temos que $y_{h}=\Phi(u, \beta)$ é a ordenada da singularidade onde o traço da matriz Jacobiana se anula.

Se substituirmos o valor de $y_{h}$ nas equações (3.13) então teremos os valores exatos da abscissa da singularidade e do parâmetro $\lambda$ para os quais o traço da matriz Jacobiana se 
anula. Assim, fixados valores para $a, b, c, d, e, f, \alpha$ e $\beta$ temos a existência de $y_{h}, x_{h}$ e $\lambda_{h}$ únicos onde o traço da matriz Jacobiana na singularidade $\left(x_{h}, y_{h}\right)$ se anula para o parâmetro $\lambda_{h}$.

Já mencionamos que as raízes da função $\Psi(u, \beta)$ são $u=0, u= \pm 8 \sqrt{3} \beta$. Além disso, essa função tem dois pontos críticos complexos conjugados e dois reais, um dos quais é negativo, o ponto crítico positivo é $u=\sqrt{-30+18 \sqrt{17}} \beta$, que vale aproximadamente $6,6495 \beta$, que é um ponto de máximo. Nesse ponto crítico a função vale $(-3085344+778464 \sqrt{17})^{1 / 3} \beta$ que é, aproximadamente, $49,9125 \beta$. Para valores de $u$ maiores que $8 \sqrt{3} \beta$ a função admite valores complexos. Logo, $\Psi(u, \beta)$ será positiva se $0<u<8 \sqrt{3} \beta$. A próxima figura contém o gráfico de $\Psi$.

Agora vamos retornar à função $\Phi(u, \beta)$. Essa função tem uma única raiz real em $u=2 \beta$, quando $u$ tende a zero pela direita a função tende a $+\infty$ e quando $u$ tende a $8 \sqrt{3} \beta$, pela esquerda, temos que $\Phi(u, \beta)$ tende a $(-4-5 \sqrt[3]{4}) /(4 \sqrt{3})$ que é aproximadamente $-1,72295$. Temos assim que se $0<u<2 \beta$ então $\Phi(u, \beta)$ é positiva, $\Phi(u, \beta)$ se anula quando $u=2 \beta$, é negativa para valores de $u$ entre $2 \beta$ e $8 \sqrt{3} \beta$ e admite valores complexos quando $u>8 \sqrt{3} \beta$. Portanto o domínio de $\Phi(u, \beta)$ é $(0,8 \sqrt{3}) \times \mathbb{R}^{+}$e a sua imagem é o conjunto $(-1,72295,+\infty)$.

Além disso, a função $\Phi(u, \beta)$ não tem pontos críticos em $0<u<8 \sqrt{3} \beta$ e aí ela é bijetora. Para ver isto basta notar que a derivada de $\Phi(u, \beta)$ em função de $u$ não muda de sinal.

A próxima figura apresenta os gráficos de $\Psi$ e $\Phi$ para um valor fixo de $\beta$.
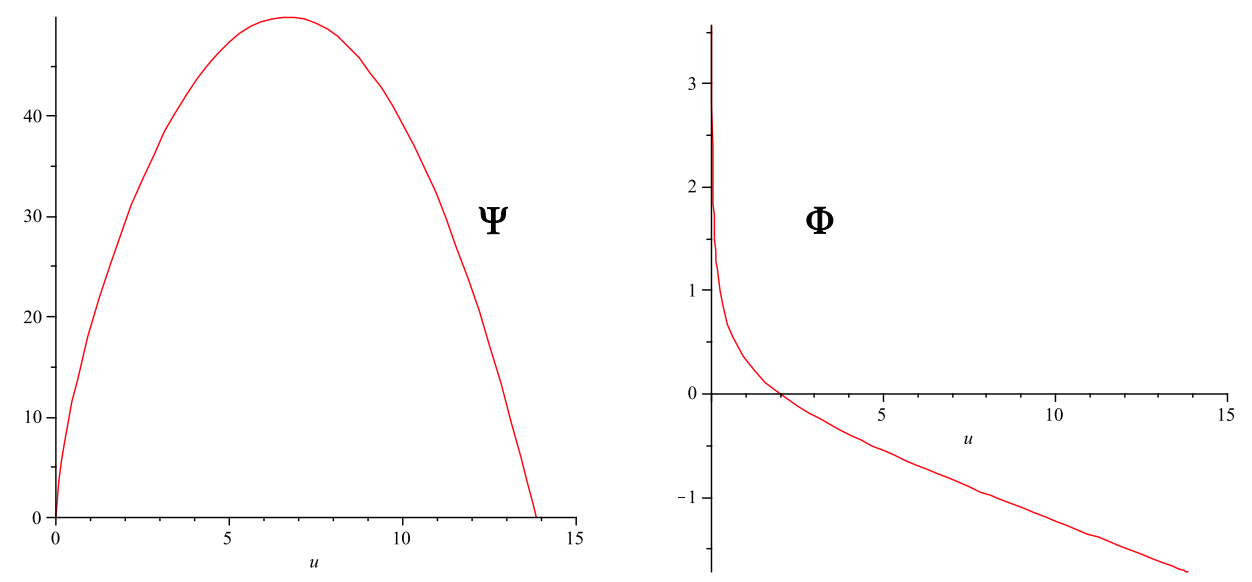

figura 10: Gráficos de $\Psi$ e $\Phi$

Para verificar que o primeiro número de Lyapunov não é nulo, basta avaliar o termo que está entre chaves na fórmula de $l_{1}$, que denotaremos por $L_{1}$, uma vez que $b$ e $\delta$ são ambos 
não-negativos. Fazendo os cálculos necessários, obtemos que

$$
\begin{aligned}
& L_{1}=\frac{c\left(\Phi+\sqrt{1+\Phi^{2}}\right)}{64\left(1+\Phi^{2}\right)^{5}}\left[12(a+3 d)\left(a^{2}+b c\right) \Phi^{5}\right. \\
& +12\left((a+3 d) \sqrt{1+\Phi^{2}}+4 \beta\right)\left(a^{2}+b c\right) \Phi^{4} \\
& +\left(\left(19 a+52 d+48 \beta \sqrt{1+\Phi^{2}}\right)\left(a^{2}+b c\right)-8 d(a d-b c)+4 b c(a+d)+72 a \beta^{2}\right) \Phi^{3} \\
& +\left(\left(36 \beta+(13 a+34 d) \sqrt{1+\Phi^{2}}\right)\left(a^{2}+b c\right)-8 d \sqrt{1+\Phi^{2}}(a d-b c)\right. \\
& \left.+4 b c \sqrt{1+\Phi^{2}}(a+d)-2 c \alpha(a+2 d)-72 a \beta^{2} \sqrt{1+\Phi^{2}}\right) \Phi^{2} \\
& +\left(\left(-18 \beta\left(a^{2}-b c\right)+2 c \alpha(a-d)-6 d(c \alpha+8 a \beta)\right) \sqrt{1+\Phi^{2}}+7 a\left(a^{2}+b c\right)\right. \\
& +8 a d(2 a-d)-36 c \alpha \beta+4 b c(a+7 d)) \Phi \\
& +\left(a\left(a^{2}+b c\right)-(8 d+2 a)(a d-b c)+2 b c(a+d)+24 c \alpha \beta\right) \sqrt{1+\Phi^{2}} \\
& \left.-12 \beta\left(a^{2}+b c\right)-2 c \alpha(a+2 d)\right] .
\end{aligned}
$$

Como, pelo Lema 11, devemos ter $a+d<\left(\frac{a d-b c}{a \beta-c \alpha}\right) \beta$, e sendo $a+d>0$ e $\beta>0$, então fixados $a, d$ e $\beta$ existe um único $0<k<\frac{a d-b c}{a \beta-c \alpha}$ tal que $a+d=k \beta$, ou ainda $a=k \beta-d$. Substituindo a por $k \beta-d$ em $\Phi$, dada por (3.33), obtemos

$$
\Phi(k \beta, \beta)=\frac{\left(\Psi^{\prime}\right)^{2}-k^{2} \Psi^{\prime}-192 k^{2}+k^{4}}{24 k \Psi^{\prime}}
$$

onde

$$
\Psi^{\prime}=\left(\left(3456-576 k^{2}-k^{4}+24\left(8+k^{2}\right) \sqrt{3 k^{2}+324}\right) k^{2}\right)^{1 / 3} .
$$

Assim, temos que $\beta$ não está mais presente na fórmula de $\Phi$.

O ponto de Hopf $\left(x_{h}, y_{h} ; \lambda_{h}\right)$ é tal que $y_{h}$ é dado por $\Phi(u, \beta)$, e vimos anteriormente que a função $\Phi(u, \beta)$ é decrescente, donde injetora. Portanto, dado um ponto $\delta$ na imagem de $\Phi$, existe uma única pré-imagem desse ponto com relação a $\Phi$. Seja

$$
\delta=\Phi(k \beta, \beta) \in \operatorname{Im}(\Phi(u, \beta))=(-1,72295,+\infty)
$$


então $\Phi(k \beta, \beta)=\delta$ se $k$ for igual a

$$
k_{ \pm}=\frac{2\left(-2 \delta-2 \delta^{3} \pm \sqrt{4 \delta^{6}+8 \delta^{4}+5 \delta^{2}+1}\right)}{1+\delta^{2}} .
$$

Porém, apenas a primeira raiz retorna valores positivos de $k$. Portanto, vamos considerar a raiz $k_{+}$.

Assim, substituindo $\Phi$ por $\delta$, e $a$ por $k_{+} \beta-d$ em $L_{1}$ obtemos

$$
\widetilde{L}_{1}=\left(F_{1} d^{3}+F_{2} d^{2} \beta+F_{3} d \beta^{2}+F_{4} b c d+F_{5} c d \alpha+F_{6} \beta^{3}+F_{7} b c \beta+F_{8} c \alpha \beta\right) c
$$

onde

$$
\begin{aligned}
F_{1} & =\frac{\left(24 \delta^{3}+24 \sqrt{1+\delta^{2}} \delta^{2}+17 \delta+5 \sqrt{1+\delta^{2}}\right)\left(\sqrt{1+\delta^{2}}+\delta\right)}{64\left(1+\delta^{2}\right)^{4}} \\
F_{2} & =-\frac{\left(\sqrt{1+\delta^{2}}+\delta\right)}{32\left(1+\delta^{2}\right)^{5}}\left[-72 \delta^{6}-72 \sqrt{1+\delta^{2}} \delta^{5}-134 \delta^{4}-98 \sqrt{1+\delta^{2}} \delta^{3}-17 \sqrt{1+\delta^{2}} \delta\right. \\
& \left.+\left(36 \delta^{3}+19 \delta\right)\left(1+2 \delta^{2}\right) \sqrt{1+\delta^{2}}-56 \delta^{2}+\left(36 \delta^{2}+1\right)\left(1+2 \delta^{2}\right)\left(1+\delta^{2}\right)+6\right] \\
F_{3} & =-\frac{\left(\sqrt{1+\delta^{2}}+\delta\right)}{16\left(1+\delta^{2}\right)^{6}}\left[\left(28 \delta^{3}-14 \delta\right)\left(1+2 \delta^{2}\right)\left(1+\delta^{2}\right)+29 \delta+15 \delta^{3}-56 \delta^{7}-70 \delta^{5}\right. \\
& \left.+\left(5-46 \delta^{4}+15 \delta^{2}-56 \delta^{6}\right) \sqrt{1+\delta^{2}}+\left(16 \delta^{2}+28 \delta^{4}-12\right)\left(1+2 \delta^{2}\right) \sqrt{1+\delta^{2}}\right] \\
F_{4} & =\frac{\left(24 \delta^{3}+\left(24 \delta^{2}+5\right) \sqrt{1+\delta^{2}}+17 \delta\right)\left(\sqrt{1+\delta^{2}}+\delta\right)}{64\left(1+\delta^{2}\right)^{4}} \\
F_{5} & =-\frac{\left(\delta^{2}+5 \sqrt{1+\delta^{2}} \delta+1\right)\left(\sqrt{1+\delta^{2}}+\delta\right)}{32\left(1+\delta^{2}\right)^{5}} \\
F_{6}= & \frac{\left(\sqrt{1+\delta^{2}}+\delta\right)}{8\left(1+\delta^{2}\right)^{6}}\left[-6-604 \delta^{6}-800 \delta^{8}-72 \delta^{2}-254 \delta^{4}-384 \delta^{10}+\left(-384 \delta^{9}-608 \delta^{7}\right.\right. \\
& \left.-364 \delta^{5}-122 \delta^{3}-15 \delta\right) \sqrt{1+\delta^{2}}+\left(192 \delta^{6}+112 \delta^{4}+46 \delta^{2}+1\right)\left(1+\delta^{2}\right)\left(1+2 \delta^{2}\right) \\
& \left.+\left(+192 \delta^{7}+208 \delta^{5}+70 \delta^{3}+31 \delta\right)\left(1+2 \delta^{2}\right) \sqrt{1+\delta^{2}}\right]
\end{aligned}
$$




$$
\begin{aligned}
F_{7} & =\frac{\left(\sqrt{1+\delta^{2}}+\delta\right)}{32\left(1+\delta^{2}\right)^{5}}\left[-24 \delta^{6}-22 \delta^{4}-4 \delta^{2}-6+\left(-24 \delta^{5}-10 \delta^{3}-\delta\right) \sqrt{1+\delta^{2}}\right. \\
& \left.+\left(12 \delta^{3}+11 \delta\right)\left(1+2 \delta^{2}\right) \sqrt{1+\delta^{2}}+\left(12 \delta^{2}+5\right)\left(1+\delta^{2}\right)\left(1+2 \delta^{2}\right)\right] \\
F_{8} & =\frac{\left(\sqrt{1+\delta^{2}}+\delta\right)}{16\left(1+\delta^{2}\right)^{6}}\left[2 \delta^{5}-5 \delta^{3}+\left(-2 \delta^{4}+4 \delta^{2}+6\right) \sqrt{1+\delta^{2}}-7 \delta-\left(\delta^{2}+1\right) \sqrt{1+\delta^{2}}\left(1+2 \delta^{2}\right)\right. \\
& \left.+\delta\left(1+\delta^{2}\right)\left(1+2 \delta^{2}\right)\right] .
\end{aligned}
$$

Assim, $\widetilde{L}_{1}$ é linear, por exemplo, em $b$ e se anula quando $b=b_{0}$ onde

$$
b_{0}=-\frac{F_{1} d^{3}+F_{2} d^{2} \beta+F_{3} d \beta^{2}+F_{8} c \alpha \beta+F_{5} c d \alpha+F_{6} \beta^{3}}{c\left(F_{7} \beta+F_{4} d\right)},
$$

onde as funções $F_{i}, i=1,2, \ldots, 8$, são dadas acima. Portanto, se $b \neq b_{0}$ então $\widetilde{L}_{1}$ não se anula, e como o primeiro número de Lyapunov $l_{1}$ é igual a $\widetilde{L}_{1}$ multiplicado por um fator que não se anula, então teremos que, nessas condições, $l_{1} \neq 0$.

Determinar o sinal de $b_{0}$ é uma tarefa árdua por depender de vários coeficientes da família original, e não pretendemos tratar desse assunto nesse trabalho. Porém, se $b_{0} \leq 0$ então o primeiro número de Lyapunov não se anula para qualquer valor de $b$, e se $b_{0}>0$ então $l_{1} \neq 0$ para $b \in \mathbb{R}^{+}-\left\{b_{0}\right\}$.

Um fato importante é que o ciclo limite gerado pela bifurcação de Hopf é único. Na seção que trata do caso $\mathcal{F}_{4}$ vamos provar que ocorre uma bifurcação de Bogdanov-Takens, sendo o caso $\mathcal{F}_{4}$ uma transição entre os casos $\mathcal{F}_{1}$ e $\mathcal{F}_{2}$. Isto mostra, através dos resultados conhecidos sobre o diagrama de bifurcação de Bogdanov-Takens, que ocorre apenas um ciclo limite no caso $\mathcal{F}_{1}$, e tal ciclo vem da bifurcação de Hopf. Portanto, a conexão de sela está associada ao caso $\mathcal{F}_{4}$ e não ocorre tal conexão no caso $\mathcal{F}_{1}$.

Assim, provamos a seguinte proposição.

Proposição 17. Seja $Z_{\lambda}=\left(X_{\lambda}, Y_{\lambda}\right)$ uma FCD dada por (3.1), que é do tipo $\mathcal{F}_{1}$. Dados $\beta>0 e$

$$
a+d<\min \left\{8 \sqrt{3} \beta, \frac{(a d-b c) \beta}{a \beta-c \alpha}\right\},
$$

seja $b_{0}$, onde $b_{0}$ é dado por (3.37) e $\delta=\Phi(a+d, \beta)$ é dado por (3.33). Se $b \neq b_{0}$ então a respectiva $F C R, Z_{\lambda, R}$, admite uma bifurcação de Hopf quando o parâmetro vale $\lambda_{h}$, e não ocorre uma conexão de sela.

Agora que já temos a bifurcação de Hopf, é de grande importância saber a estabilidade do ponto de Hopf, ou seja, descobrir o sinal do primeiro número de Lyapunov. Usando as 
regras de regularização de um campo descontínuo, que enunciamos no primeiro capítulo, podemos esperar que a regularização do caso $\mathcal{F}_{1}$ contenha um ponto de Hopf com primeiro número de Lyapunov negativo. Vamos verificar essa afirmação.

Vamos iniciar encontrando a região do plano $(a, d)$ que vamos considerar.

Lema 18. Seja $Z_{\lambda}$ uma $F C D$ do tipo $\mathcal{F}_{1}$. Então, existe um subconjunto, $A$, aberto no plano $(a, d)$ onde estão definidos os coeficientes a e $d$ de $Z_{\lambda}$.

Demonstração. O subconjunto $A$ que estamos procurando é a interseção das regiões contidas no plano $(a, d)$, caracterizadas por $0<a+d<8 \sqrt{3} \beta$, ad $-b c>0, G(a, b, c, d)<0$, $(a-d)^{2}+4 b c<0, a+d<\frac{(a d-b c) \beta}{a \beta-c \alpha}$ e $a>0$.

Em primeiro lugar, notamos que a região do plano $(a, d)$ que procuramos está limitada pela esquerda pela reta $a+d=0$, e pela direita pela reta $a+d=8 \sqrt{3} \beta$. Além disso, temos que a região de $(a, d)$ onde vale $a d-b c>0$ é o plano todo. De fato, se $a d-b c=w$ onde $0<w<-b c$ então $a d-b c-w=0$ está contido no segundo e quarto quadrantes. Por outro lado, se $a d-b c=w$ onde $w>-b c$ então $a d-b c-w=0$ está contido no primeiro e terceiro quadrantes. Mas, como $a+d>0$ então o terceiro quadrante está fora da nossa região, ou seja, a região que estamos interessados está contida nos quadrantes restantes.

A função $G(a, b, c, d)$ está definida para valores de $a$ positivo. O gráfico de $G(a, b, c, d)=0$ tem duas componentes conexas, uma contida no segundo quadrante, que recai sobre a curva $a d-b c=0$, e a segunda componente está no primeiro quadrante. Esta componente do primeiro quadrante intersecta a diagonal $a=d$ no ponto $d=0,47454 \sqrt{-b c}$ e tende a $d=+\infty$ quando $a$ tende a zero. Por outro lado, os pontos onde $G(a, b, c, d)<0$ ficam à esquerda da componente conexa, do primeiro quadrante, descrita acima, e abaixo da reta $a=d$, e acima da curva $a d-b c=0$ no segundo quadrante. Portanto, as curvas $G(a, b, c, d)=0$ e $a=d$ contidas no primeiro e segundo quadrantes fazem parte do bordo da região que procuramos, isto pois, pela definição da função $G(a, b, c, d)$, devemos desconsiderar a reta $a=d$.

Quanto à desigualdade $(a-d)^{2}+4 b c<0$, devemos notar que dado $m<0$ então $(a-d)^{2}+$ $4 b c=m$ faz sentido apenas se $m>4 b c$. E neste caso, a desigualdade $(a-d)^{2}+4 b c<0$ nos dá duas retas paralelas entre si e perpendiculares à reta $a+d=0$. Além disso, uma dessas retas encontra a reta $a+d=0$ no ponto $(\sqrt{-b c},-\sqrt{-b c})$ e a outra no ponto $(-\sqrt{-b c}, \sqrt{-b c})$. A região do plano onde vale $(a-d)^{2}+4 b c<0$, está contida entre essas duas retas.

Para finalizar vamos encontrar a região do plano pertinente à desigualdade $a+d<$ $\frac{(a d-b c) \beta}{a \beta-c \alpha}$. Manipulando essa desigualdade obtemos $\left(a^{2}+b c\right) \beta<(a+d) c \alpha$ agora, como 
$c(a+d)<0$ temos então que

$$
\frac{\alpha}{\beta}<\frac{a^{2}+b c}{c(a+d)} .
$$

Notamos que se $\alpha / \beta$ fosse nulo então o gráfico implícito de $\frac{a^{2}+b c}{c(a+d)}$ em $(a, d)$ seriam duas retas verticiais em $a= \pm \sqrt{-b c}$. Por outro lado, para um valor fixo de $\alpha / \beta=m>0$ temos que $\frac{a^{2}+b c}{c(a+d)}=m$ implica que $a^{2}+b c=c(a+d) m$. Portanto, a curva de $a^{2}+b c=c(a+d) m$ intersecta a reta $a+d=0$ nos pontos $a= \pm \sqrt{-b c}$, ou seja, nas retas descritas anteriormente. Se nos restringimos ao primeiro quadrante então temos que fixado o valor de $b c$, à medida que aumentamos o valor de $\alpha / \beta$, os valores de interseção entre o eixo vertical e o gráfico de $\frac{a^{2}+b c}{c(a+d)}$ vai tendendo a zero, isto pois esses pontos de interseção são dados por $b / m$ onde $m=\alpha / \beta$. E o mesmo ocorrre com a interseção com o eixo horizontal onde os pontos de interseção são dados por $\left(c m+\sqrt{c^{2} m^{2}-4 b c}\right) / 2$. Portanto, a região caracterizada por $a+d<\frac{(a d-b c) \beta}{a \beta-c \alpha}$ está contida entre o eixo vertical e a reta vertical em $a=\sqrt{-b c}$. Isso finaliza a prova do lema.

A próxima figura apresenta um exemplo do comportamento da região $a+d<\frac{(a d-b c) \beta}{a \beta-c \alpha}$ no plano $(a, d)$. Estamos considerando que $b c=-1, c=-2$ além disso temos três valores distintos de $\alpha / \beta$ que são 0,1 e 3 .

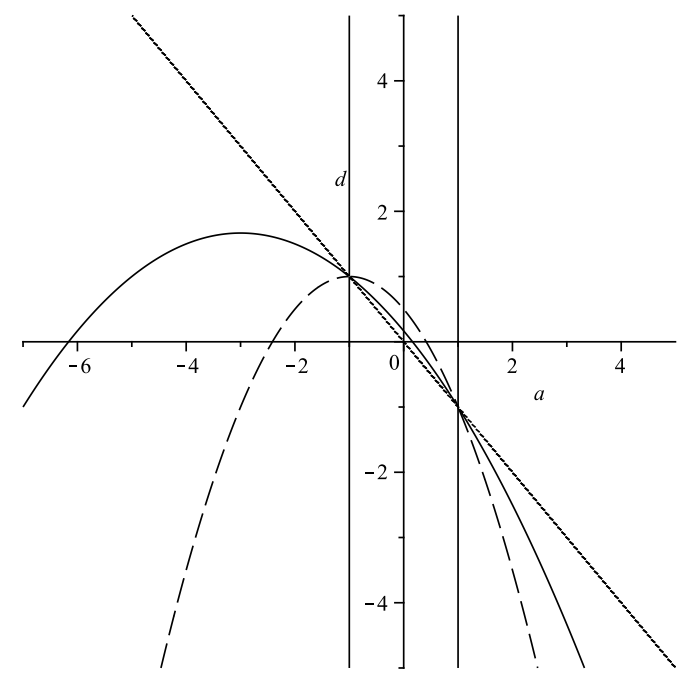

figura 11: Região onde valem $a$ e $d$, para $b c=-1$.

Um exemplo da região descrita pelo Lema anterior, onde estamos supondo que $b c=-1$ 
pode ser visto na próxima figura em hachurado. Note que o subconjunto hachurado tem duas componentes conexas, separadas pela reta $a=d$.

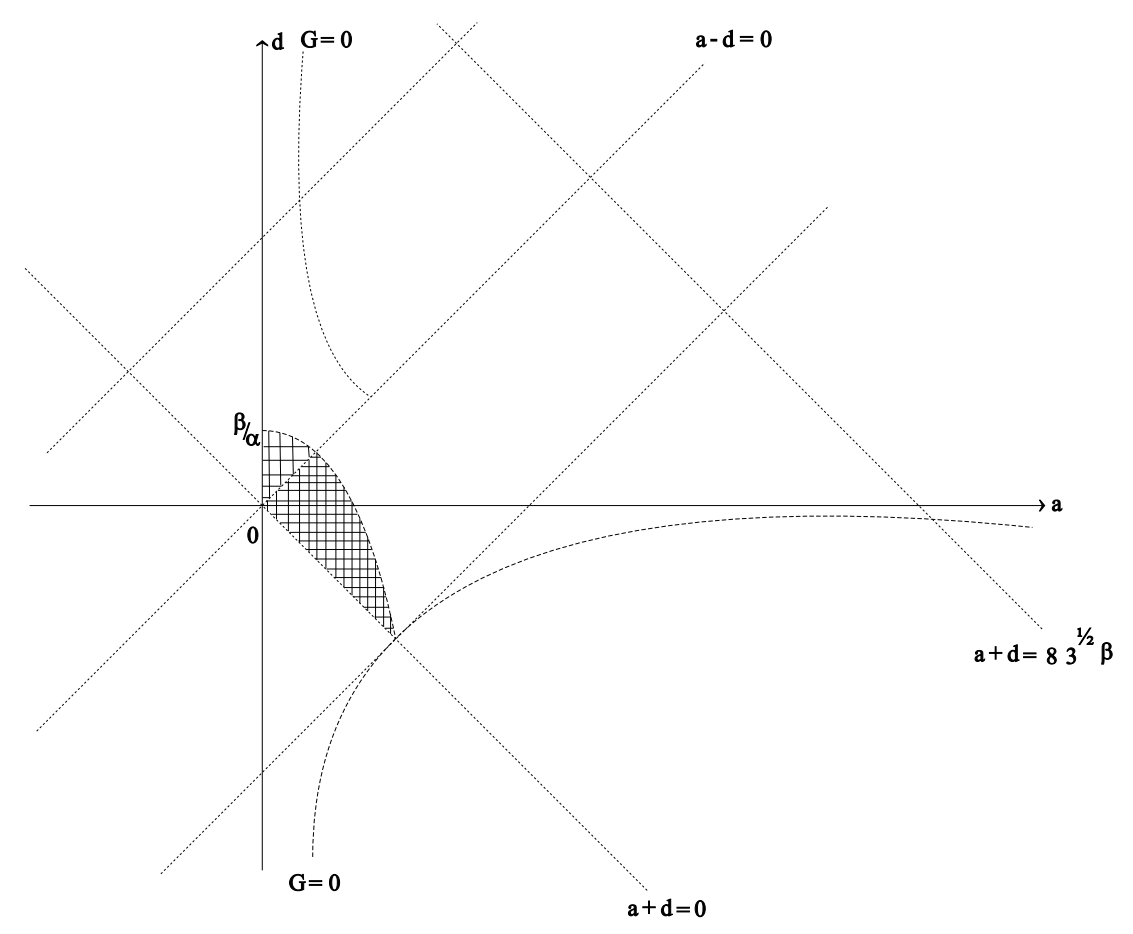

figura 12: Região onde valem $a$ e $d$, para $b c=-1$. 
A próxima proposição trata do sinal do primeiro número de Lyapunov. Notamos que o lema anterior não nos deu elementos suficientes para restringir o valor de $a+d$ em $(0,8 \sqrt{3} \beta)$. Assim, precisamos fazer mais hipóteses sobre os coeficientes de $Z_{\lambda}$ para determinarmos o sinal de $l_{1}$.

Proposição 19. Seja $Z_{\lambda}$ uma $F C D$ do tipo $\mathcal{F}_{1}$. Vamos supor que $b \neq b_{0}$, onde $b_{0}$ é dado por (3.37) e $\delta=\Phi(a+d, \beta)$ é dado por (3.33), e vamos considerar que $d F_{4}+\beta F_{7} \neq 0$, onde $F_{4}$ e $F_{7}$ são dados na definição de (3.36). Então, a família regularizada, $Z_{\lambda, R}$, admite um ponto de Hopf cujo primeiro número de Lyapunov é negativo em qualquer um dos seguintes casos

1) se $0<a+d \leq 1,69 \beta, d>0$ com $b_{0} \leq 0$ e $b>0$ ou com $b_{0}>0$ e $b>b_{0}$.

2) $\operatorname{sejam} 1,69 \beta<a+d \leq 8,4 \beta$ e $d>0$

2.1) $s e \frac{F_{4}}{F_{7}}<-\frac{\beta}{d}$ com $b_{0} \leq 0$ e b $>0$ ou com $b_{0}>0$ e $b>b_{0}$.

2.2) $s e \frac{F_{4}}{F_{7}}>-\frac{\beta}{d}$ com $b_{0}>0$ e $0<b<b_{0}$.

3) se $8,4 \beta<a+d<8 \sqrt{3} \beta$ e $d>0$ com $b_{0}>0$ e $0<b<b_{0}$.

4) $\operatorname{seja} d=0$

4.1) se $0<a<1,69 \beta$ com $b_{0} \leq 0$ e $b>0$ ou com $b_{0}>0$ e $b>b_{0}$.

4.2) se $a>1,69 \beta$ com $b_{0}>0$ e $0<b<b_{0}$.

5) se $0<a+d \leq 1,69 \beta, d<0$

5.1) $s e \frac{F_{4}}{F_{7}}<-\frac{\beta}{d}$ com $b_{0} \leq 0$ e b $>0$ ou com $b_{0}>0$ e $b>b_{0}$.

5.2) $s e \frac{F_{4}}{F_{7}}>-\frac{\beta}{d}$ com $b_{0}>0$ e $0<b<b_{0}$.

6) sejam $1,69 \beta<a+d \leq 8,4 \beta$ e $d<0$

6.1) se $\frac{F_{4}}{F_{7}}>-\frac{\beta}{d}$ com $b_{0} \leq 0$ e $b>0$ ou com $b_{0}>0$ e $b>b_{0}$.

6.2) $s e \frac{F_{4}}{F_{7}}<-\frac{\beta}{d}$ com $b_{0}>0$ e $0<b<b_{0}$.

7) se $8,4 \beta<a+d<8 \sqrt{3} \beta$ e $d<0$

7.1) $s e \frac{F_{4}}{F_{7}}>-\frac{\beta}{d}$ com $b_{0} \leq 0$ e $b>0$ ou com $b_{0}>0$ e $b>b_{0}$. 
7.2) $s e \frac{F_{4}}{F_{7}}<-\frac{\beta}{d}$ com $b_{0}>0$ e $0<b<b_{0}$.

Demonstração. Para provar o resultado, devemos verificar que $\widetilde{L}_{1}>0$, onde $\widetilde{L}_{1}$ é dado por (3.36), uma vez que o primeiro número de Lyapunov é um fator negativo multiplicado por $\widetilde{L}_{1}$. Assim, para quaisquer valores de $b$ temos que a derivada de $\widetilde{L}_{1}$ em função de $b$ é dada por

$$
\frac{\partial \widetilde{L}_{1}}{\partial b}=\left(d F_{4}+\beta F_{7}\right) c^{2}
$$

que é diferente de zero por hipótese. Portanto, o sinal de (3.38) em conjunto com o sinal de $b_{0}$ indicará onde $\widetilde{L}_{1}$ é positivo ou negativo. Ou seja, temos os seguintes casos possíveis:

I) $\operatorname{seja} \frac{\partial \widetilde{L}_{1}}{\partial b}>0$

I.1) se $b_{0} \leq 0$ então $\widetilde{L}_{1}>0$ para todo valor de $b>0$, veja gráfico da esquerda da figura 13;

I.2) se $b_{0}>0$ então $\widetilde{L}_{1}>0$ para todo valor de $b>b_{0}$, e $\widetilde{L}_{1}<0$ para todo valor de $0<b<b_{0}$, veja gráfico da direita da figura 13 .
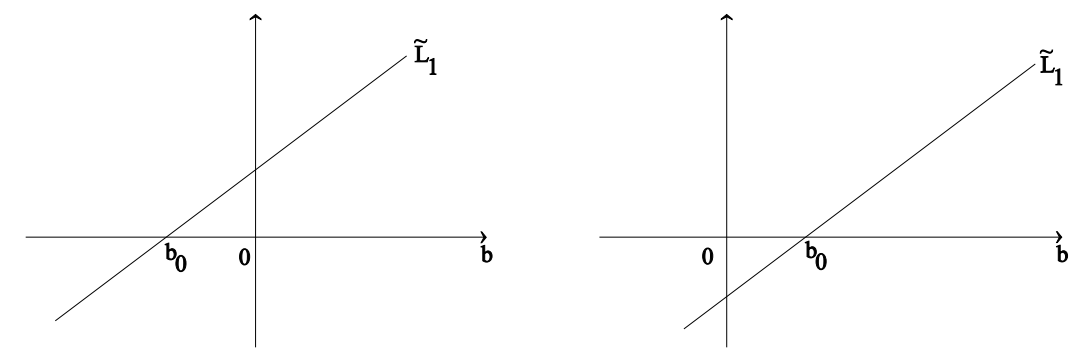

figura 13: Gráfico de $\widetilde{L}_{1}$

II) $\operatorname{seja} \frac{\partial \widetilde{L}_{1}}{\partial b}<0$

II.1) se $b_{0} \leq 0$ então $\widetilde{L}_{1}>0$ para nenhum valor de $b>0$, veja gráfico da esquerda da figura 14

I.2) se $b_{0}>0$ então $\widetilde{L}_{1}>0$ para todo valor de $0<b<b_{0}$, e $\widetilde{L}_{1}<0$ para todo valor de $b>b_{0}$, veja gráfico da direita da figura 14 .

Vamos analisar o sinal de (3.38). Para isto, uma vez que já temos a região que define $d$, dada pelo Lema 18, precisamos estudar as funções $F_{4}$ e $F_{7}$, ambas definidas em $(-1,72295,+\infty)$. 

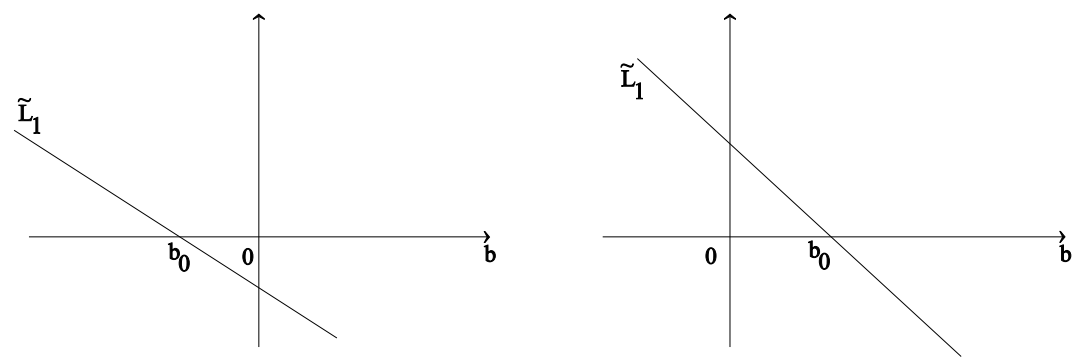

figura 14: Gráfico de $\widetilde{L}_{1}$

Observamos que $F_{4}$ se anula somente em $\delta_{0}=-5 \sqrt{6} / 12 \approx-1,02062$, os pontos $\delta_{1,2}=$ $\mp \frac{\sqrt{12690 \pm 210 \sqrt{1281}}}{120}$ que valem, aproximadamente, $\delta_{1}=0,59941$ e $\delta_{2}=-1,18456$ são os pontos críticos de $F_{4}$. Além disso, temos que $\delta_{1}$ é um ponto de máximo local, e $\delta_{2}$ um ponto de mínimo local. Para valores de $\delta$ maiores que $\delta_{0}$ a função $F_{4}$ é positiva e tende a zero quando $\delta$ tende a $+\infty$, e vale, aproximadamente, $-258 \times 10^{-8}$ quando $\delta=-1,72295$. A próxima figura exemplifica os fatos acima.

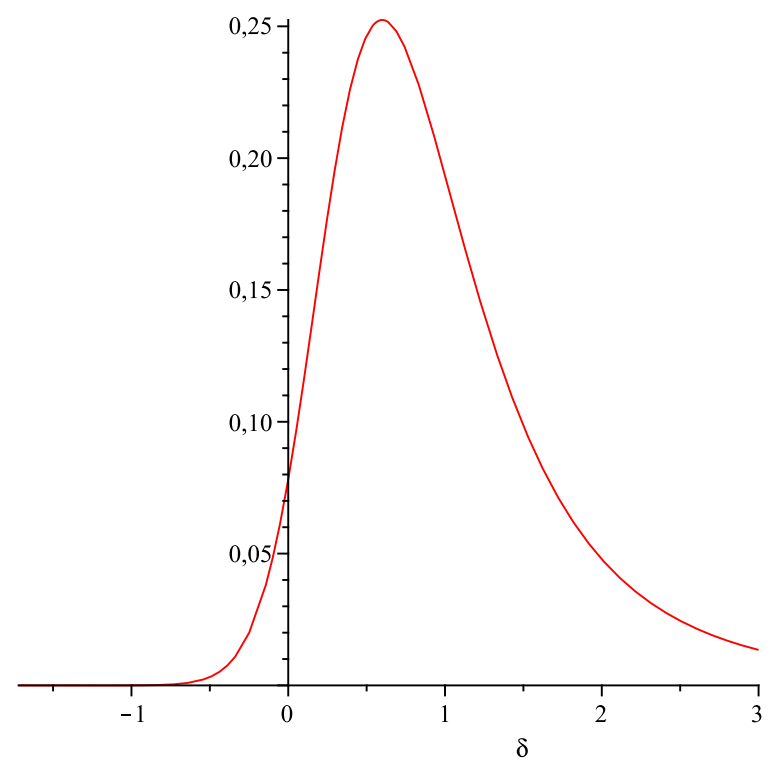

figura 15: Gráfico de $F_{4}$

A função $F_{7}$ se anula quando $\delta$ vale $\delta_{3}=\sqrt{882-66 \sqrt{177}} / 24$ ou $\delta_{4}=-\sqrt{882+66 \sqrt{177}} / 24$ que valem, aproximadamente, 0,08257 e $-1,74805$, respectivamente. Portanto, $F_{7}$ se anula em um ponto fora do domínio que especificamos para essa função, ou seja, $\delta_{4}$ não nos inter- 
essa. Além disto, os pontos $\delta_{5}=-\frac{\sqrt{3}}{12} \approx-0,144337$,

$$
\delta_{6,7}=-\frac{14(130455 \mp 27410 \sqrt{19})^{3 / 2}}{321039625}+\frac{444763}{64207925} \sqrt{130455 \mp 27410 \sqrt{19}}
$$

que valem, aproximadamente, $\delta_{6}=0,675603$ e $\delta_{7}=-1,985839$ são pontos críticos de $F_{7}$. O ponto $\delta_{7}$ não nos interessa, por estar fora do domínio de $F_{7}$. Pelo teste da derivada segunda temos que $\delta_{5}$ é um ponto de mínimo local e $\delta_{6}$ é um ponto de máximo local. Se $\delta<\delta_{3}$ então $F_{7}<0$ e será positivo se $\delta>\delta_{3}$, e $F_{7}$ tende a zero, por valores positivos, quando $\delta$ tende a $+\infty$. A próxima figura apresenta o gráfico de $F_{7}$.

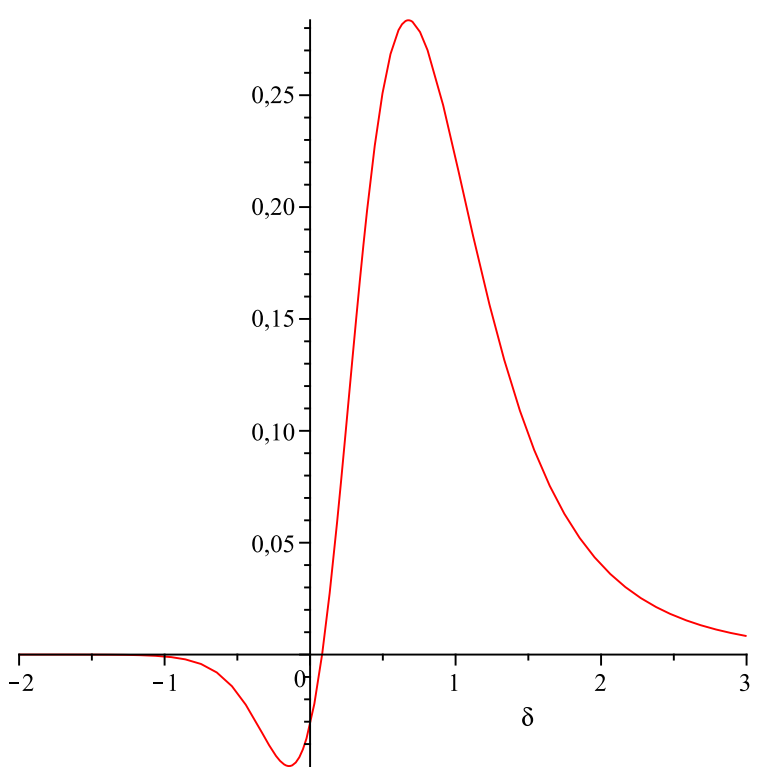

figura 16: Gráfico de $F_{7}$

Supondo que $d>0$ e $a>0$ temos que se $\delta>\delta_{3}$ então $F_{7}$ é positivo, e $F_{4}$ também o é. Por outro lado, o ponto $u_{3}=1,69 \beta$ é tal que $\Phi\left(u_{3}, \beta\right)=\delta_{3}$. Portanto, se $0<a+d \leq 1,69 \beta$ então temos que (3.38) é estritamente positivo. Portanto, $l_{1}<0$ se $b_{0} \leq 0$ e $b>0$ ou se $b_{0}>0$ e $b>b_{0}$. O caso em que $d<0$ temos que se $\frac{F_{4}}{F_{7}}<-\frac{\beta}{d}$ então $l_{1}<0$ se $b_{0} \leq 0$ e $b>0$ ou se $b_{0}>0$ e $b>b_{0}$. E se $\frac{F_{4}}{F_{7}}>-\frac{\beta}{d}$ então $l_{1}<0$ se $b_{0}>0$ e $0<b<b_{0}$.

Agora, seja $\delta_{0}<\delta<\delta_{3}$. Temos que $u_{0}=8,4 \beta$ é tal que $\Phi\left(u_{0}, \beta\right)=\delta_{0}$. Assim, temos dois casos possíveis para o sinal de (3.38) e, consequentemente, para o sinal do primeiro número de Lyapunov. Os casos são

a) se $1,69 \beta<a+d<8,4 \beta$ e $\frac{F_{4}}{F_{7}}<-\frac{\beta}{d}$ então (3.38) é positivo donde concluimos que 
$l_{1}<0$ se $b_{0} \leq 0$ e $b>0$ ou se $b_{0}>0$ e $b>b_{0}$.

b) se $1,69 \beta<a+d<8,4 \beta$ e $\frac{F_{4}}{F_{7}}>-\frac{\beta}{d}$ então (3.38) é negativo e $l_{1}$ será negativo se $b_{0}>0$ e $0<b<b_{0}$.

Seja $-1,77295<\delta<\delta_{0}$, ou seja, $8,4 \beta<a+d<8 \sqrt{3} \beta$. Então temos que $F_{4}$ e $F_{7}$ são ambos negativos, implicando que (3.38) é negativo. Portanto, $l_{1}$ será negativo se $b_{0}>0$ e $0<b<b_{0}$. Os casos em que $d>0$ ou $d<0$ são derivados dos casos descritos acima. Além disso, se $d=0$ então (3.38) dependerá somente do sinal de $F_{7}$ e a análise feita acima nos dá o resultado procurado para este caso.

Obs.: Na Proposição 19 estamos considerando que a derivada de $\widetilde{L}_{1}$ em função de $b$ é não nula. Quando essa derivada é nula podemos considerar a variável $\alpha$, pois $\widetilde{L}_{1}$ também é linear com relação a $\alpha$, e obter uma proposição análoga à Proposição 19. Agora, se ambas as derivadas forem nulas, ou seja, se $\frac{\partial \widetilde{L}_{1}}{\partial b}=\frac{\partial \widetilde{L}_{1}}{\partial \alpha}=0$ então $\widetilde{L}_{1}$ se reduz a $\left(F_{1} d^{3}+F_{2} d^{2} \beta+\right.$ $\left.F_{3} d \beta^{2}+F_{6} \beta^{3}\right) c$, e a ideia aqui é ainda obter uma proposição análoga à Proposição 19, mas ao invés de considerarmos duas funções $F_{i}$, agora temos quatro dessas funções, donde a análise se torna mais delicada e extensa, por este motivo preferimos não mostrar esse caso.

A unificação dos resultados até agora obtidos para uma FCD do tipo $\mathcal{F}_{1}$ estão contidas no próximo teorema. No enunciado do próximo teorema os parâmetros $\lambda^{*}, \lambda_{h}$ e $\lambda_{\Delta}$ estão associados aos respectivos valores de $y^{*}, y_{h}$ e $y_{\Delta}$. Onde $y^{*}$ é dado por (3.18), $y_{h}$ por (3.30).

Teorema 20. Seja $Z_{\lambda}$ uma FCD do tipo $\mathcal{F}_{1}$. Sejam

$$
a+d<\min \left\{\frac{(a d-b c) \beta}{a \beta-c \alpha}, 8 \sqrt{3} \beta\right\},
$$

$b \neq b_{0}$, onde $b_{0}$ é dado por (3.37) e $\delta=\Phi(a+d, \beta)$ é dado por (3.33). Então, para valores do parâmetro em I, onde I é um intervalo que contém no seu interior os valores $\lambda_{h} e \lambda^{*}, a$ respectiva família de campos regularizados, $Z_{\lambda, R}$, possui uma bifurcação de Hopf em $\lambda_{h}$, uma bifurcação do tipo sela-nó quando o parâmetro vale $\lambda^{*}$, e a singularidade muda de foco para nó em $\lambda_{\Delta}$.

A próxima figura apresenta o diagrama de bifurcação da regularização de uma família descontínua que pertença ao caso $\mathcal{F}_{1}$. Assim, para cada valor fixo de $\lambda$ menor que $\lambda_{h}$ coexistem uma sela, uma órbita periódica atratora e no interior da região limitada, que tem a órbita periódica como bordo, um foco repulsor. À medida que o parâmetro vai se aproximando de $\lambda_{h}$ a órbita periódica vai diminuindo até que ela colide com o foco repulsor, exatamente 
quando o parâmetro vale $\lambda_{h}$, mudando a sua estabilidade, ou seja, a partir daí o foco se torna atrator. Quando $\lambda=\lambda_{\Delta}$ ocorre a mudança de foco atrator para nó atrator e quando o parâmetro atinge o valor $\lambda^{*}$ temos aí um ponto sela-nó, a partir desse valor não existem mais singularidades. 


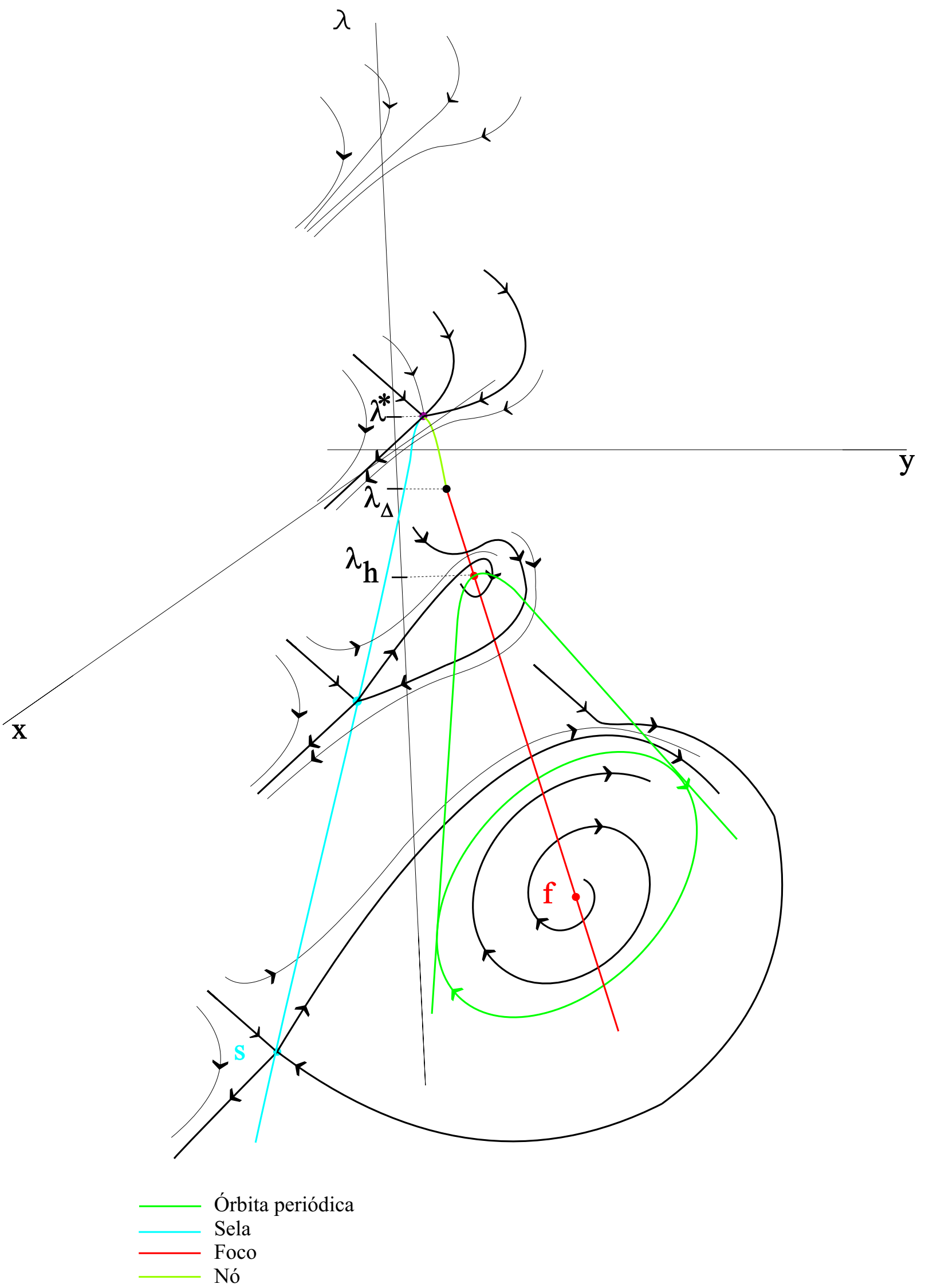

figura 17: Diagrama de bifurcação da família regularizada, referente ao caso $\mathcal{F}_{1}$. 
Obs.: A curva de Hopf no plano $(\varepsilon, \lambda)$ é, pelos mesmos motivos que a curva sela-nó, uma reta cuja inclinação é dada pelo valor do parâmetro onde ocorre a bifurcação de Hopf.

Ex.: Um exemplo de uma curva de sela-nó e Hopf no plano $(\varepsilon, \lambda)$ é dado na figura seguinte onde supomos que $\alpha=1, a=3, b=4,9, c=-2,6, d=3,5, e=2$, e $f=1$. Nesse exemplo temos que $l=5,6, m=-8,2 n=23,24$ e $u=6,5$. Além disso, $y^{*}=-0,416$, aproximadamente, $\lambda^{*}=-0,3557$ e $\lambda_{0}=-0,628$.

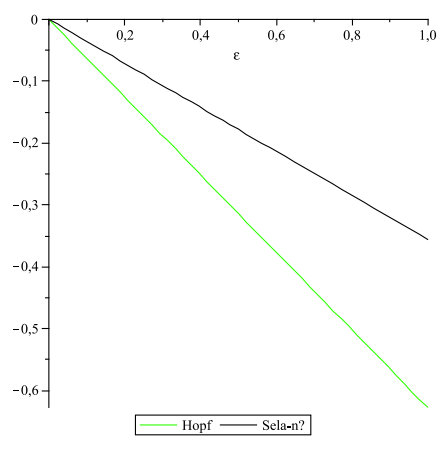

figura 18: Gráfico das curvas sela-nó e Hopf

\subsection{Bifurcação de $\mathcal{F}_{2}$ via regularização}

Do que foi visto nas seções anteriores obtemos o seguinte resultado.

Teorema 21. Seja $Z_{\lambda}$ uma FCD do tipo $\mathcal{F}_{2}$. Sejam

$$
a+d<\frac{(a d-b c) \beta}{a \beta-c \alpha}
$$

e I um intervalo que contém o parâmetro $\lambda^{*}$. Então, para valores do parâmetro em $I, a$ respectiva família de campos regularizados $Z_{\lambda, R}$ possui uma bifurcação do tipo sela-nó quando o parâmetro vale $\lambda^{*}$, e a singularidade muda de foco para nó em $\lambda_{\Delta}$.

A próxima figura apresenta o diagrama de bifurcação da regularização de uma família descontínua que pertença ao caso $\mathcal{F}_{2}$. 


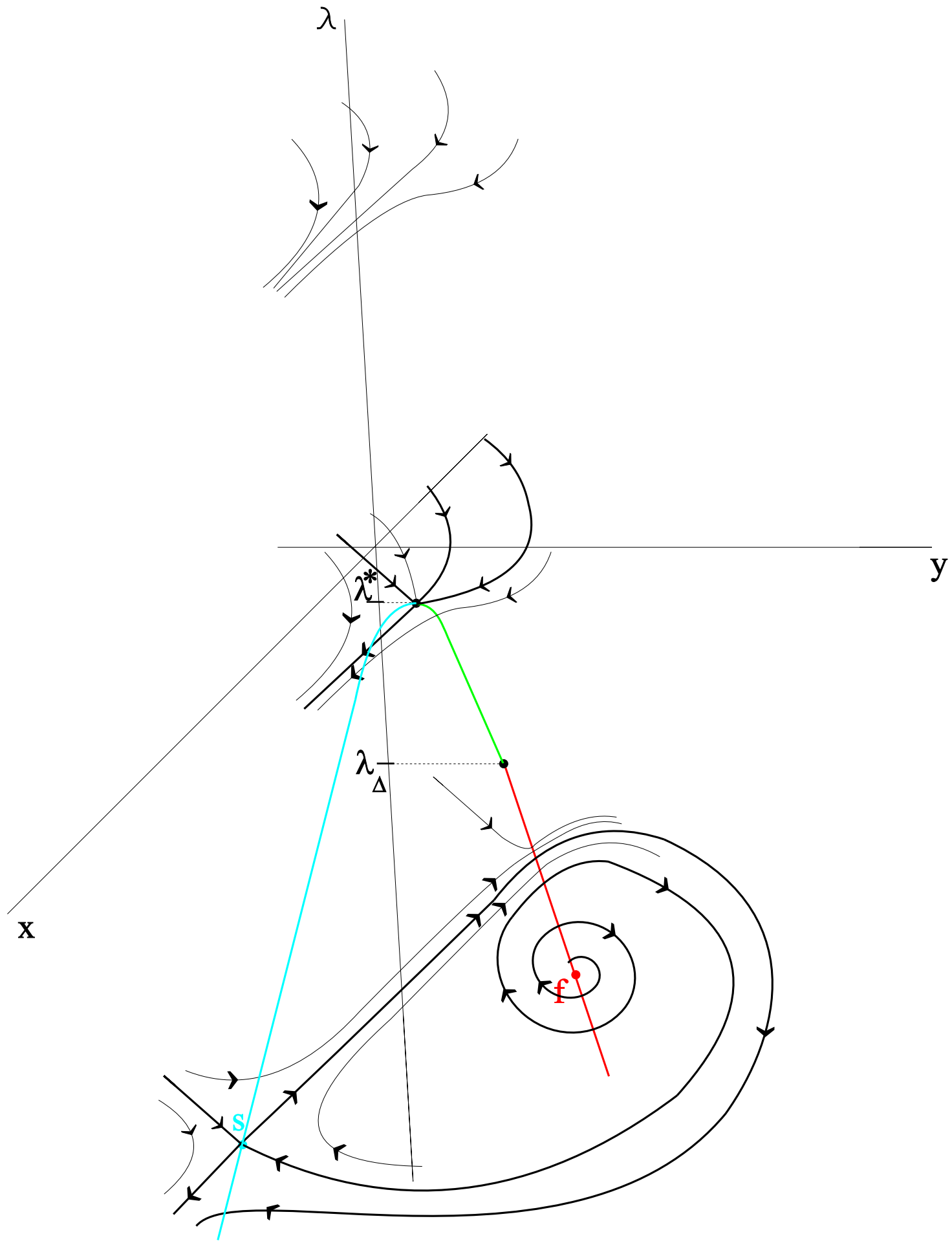

Sela

Foco

Nó

figura 19: Diagrama de bifurcação da família regularizada, referente ao caso $\mathcal{F}_{2}$. 
Obs.: Voltando às variáveis e parâmetro originais, temos que a curva sela-nó para esse caso é idêntica à curva apresentada na seção anterior, considerando o plano $(\varepsilon, \lambda)$.

\subsection{Bifurcação de $\mathcal{F}_{4}$ via regularização}

Nesta seção iremos estudar as bifurcações de uma família de campos descontínuos pertencente ao caso $\mathcal{F}_{4}$ do Lema 11 , ou seja, o caso intermediário entre os casos $\mathcal{F}_{1}$ e $\mathcal{F}_{2}$.

Inicialmente notamos que os casos intermediários entre $\mathcal{F}_{1}$ e $\mathcal{F}_{3}$ e entre $\mathcal{F}_{2}$ e $\mathcal{F}_{3}$ não serão estudados pois para a passagem a estes casos devemos trabalhar em um domínio nãocompacto, o que não é o nosso caso. Por exemplo, para passar do caso $\mathcal{F}_{1}$ ao $\mathcal{F}_{3}$ devemos fazer com que a sela de Filippov do caso $\mathcal{F}_{1}$ seja deslocada para o infinito para então obtermos o caso $\mathcal{F}_{3}$.

Lembramos que uma família $Z_{\lambda}=\left(X_{\lambda}, Y_{\lambda}\right)$ onde $X_{\lambda}(x, y)=(a x+b y+e \lambda, c x+d y+f \lambda)$, $Y(x, y)=(\alpha, \beta)$ pertence ao caso $\mathcal{F}_{4}$ se, $a+d>0, a d-b c>0, c<0,(a-d)^{2}+4 b c<0$, $c e-a f<0, \alpha>a \beta / c$ e $G(a, b, c, d)=0$, onde $G: \mathbb{R}^{4} \rightarrow \mathbb{R}$ é dada por

$$
G(a, b, c, d)=\frac{a+d}{\Delta} \operatorname{arctg}\left(\frac{\Delta}{d-a}\right)+\ln \left(\frac{a}{\sqrt{-b c}}\right)=0,
$$

onde $\Delta=\sqrt{-(a-d)^{2}-4 b c}, a>0$ e $a \neq d$.

Vamos provar que existe um único ponto de Bogdanov-Takens para a regularização de uma família descontínua que pertença ao caso $\mathcal{F}_{4}$. Esse ponto é uma singularidade de $Z_{\lambda}$ onde os autovalores da matriz Jacobiana se anulam, sem que essa matriz seja nula. Após isso calcularemos os seguintes autovetores e autovetores generalizados da matriz Jacobiana e de sua transposta calculadas no ponto de Bogdanov-Takens, $A q_{0}=0, A q_{1}=q_{0}, A^{T} p_{1}=0$ e $A^{T} p_{0}=p_{1}$ satisfazendo $<p_{0}, q_{0}>=<p_{1}, q_{1}>=1$ e $<p_{0}, q_{1}>=<p_{1}, q_{0}>=0$. Por fim, de acordo com o próximo teorema, veja $[\mathrm{K}]$, a família regularizada terá a seguinte forma normal dada por Bogdanov,

$$
\begin{aligned}
& x^{\prime}=y \\
& y^{\prime}=\beta_{1}+\beta_{2} x+\xi_{1} x^{2}+\xi_{2} x y .
\end{aligned}
$$

Teorema 22 (Bogdanov-Takens). Seja $X_{\lambda}(x, y)$ um campo vetorial no plano onde $\lambda \in \mathbb{R}^{2}$, que tem uma singularidade $\left(x_{0}, y_{0}\right)$ com respectivos parâmetros $\lambda(0)$ onde a parte linear do campo tem dois autovalores nulos. Assuma ainda que

BT.0) a matriz Jacobiana A calculada em $\left(x_{0}, y_{0} ; \lambda(0)\right)$ não se anula 
BT.1) $\xi_{1}=\frac{<p_{1}, B\left(q_{0}, q_{0}\right)>}{2} \neq 0$

BT.2) $\xi_{2}=<p_{0}, B\left(q_{0}, q_{0}\right)>+<p_{1}, B\left(q_{0}, q_{1}\right)>\neq 0$

BT.3) a transformação

$$
(x, y ; \lambda) \mapsto\left(X_{\lambda}(x, y), \operatorname{det}(A), \operatorname{tr}(A)\right)
$$

é regular no ponto $(x, y ; \lambda)=\left(x_{0}, y_{0} ; \lambda_{0}\right)$, ou seja, o determinante da parte linear da transformação calculada em $\left(x_{0}, y_{0} ; \lambda_{0}\right)$ não se anula.

Então o campo $X_{\lambda}(x, y)$ admite uma bifurcação de Bogdanov-Takens exatamente no ponto $\left(x_{0}, y_{0} ; \lambda_{0}\right)$.

O termo $B(x, y)$ usado no enunciado do teorema acima é o termo de ordem 2 da série de Taylor do campo $X_{\lambda}$, ou seja, $B(x, y)=\left(B_{1}(x, y), B_{2}(x, y)\right)$ onde $x=\left(x_{1}, x_{2}\right), y=\left(y_{1}, y_{2}\right)$ e

$$
B_{i}(x, y)=\left.\sum_{j, k=1}^{2} \frac{\partial^{2} X_{\lambda}(\zeta)}{\partial \zeta_{j} \partial \zeta_{k}}\right|_{\zeta=\zeta_{0}} x_{j} y_{k}
$$

para $i=1,2$.

Antes de comprovarmos esse teorema para o caso $\mathcal{F}_{4}$, precisamos verificar a região onde $a$ e $d$ estão definidos. Conforme a prova do Lema 18 temos que a região do plano $(a, d)$ que procuramos está contida no primeiro e segundo quadrante, mais especificamente, nas componentes conexas de $G(a, b, c, d)=0$. O gráfico seguinte apresenta exemplos dessa região. Afirmação: Seja $Z_{\lambda}$ uma FCD do tipo $\mathcal{F}_{4}$ então a derivada da função $G(a, b, c, d)$ em função de $d$ não se anula para valores de $d<0$.

Vamos provar essa afirmação através da análise dos gráficos das funções envolvidas, isto porque se tratam de funções transcendentes com expressões difíceis de manipular. A derivada parcial de $G(a, b, c, d)$ em função de $d$ vale

$$
\frac{\partial G}{\partial d}(a, b, c, d)=-\frac{1}{\Delta} \operatorname{arctg} \frac{\Delta}{d-a}-\frac{a^{2}-d^{2}}{\left(-(a-d)^{2}-4 b c\right)^{3 / 2}}+\frac{a^{2}+d^{2}}{(a-d)^{2}+4 b c} .
$$

O gráfico seguinte nos dá uma ideia de onde a função $G(a, b, c, d)$ se anula, ali estamos considerando o gráfico implícito de $G$, em $a$ e $d$, e supondo que $b c=k$ onde $k$ toma quatro valores distintos, a saber, $\{-0,05,-0,5,-1,-1,5\}$. Os gráficos têm duas componentes conexas, e para $d$ negativo a componente conexa que vai se aproximando dos eixos está relacionada com a função cujo $k$ vai tendendo a zero, o mesmo se dá para a outra componente conexa. 


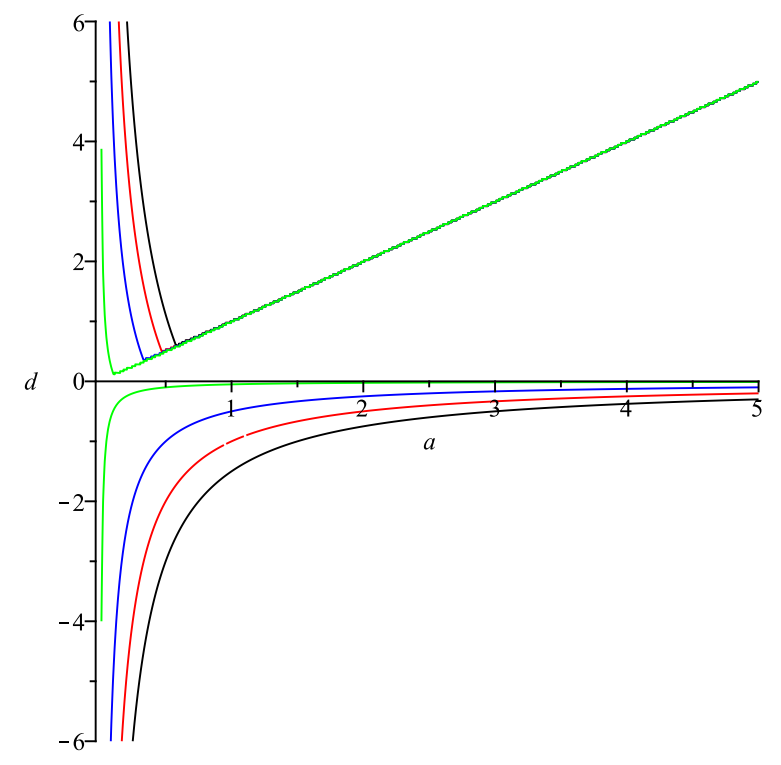

figura 20: Gráfico de $G=0$ para diferentes valores de $b c$

Por outro lado, o gráfico de $\frac{\partial G}{\partial d}(a, b, c, d)=0$, implicitamente em $a$ e $d$, é dado pela figura seguinte. A reta $a=d$ não é considerada pois estamos supondo, por hipótese, que $a \neq d$. As cores seguem os valores de $b c$ conforme os diferentes casos da figura anterior. Note que a derivada de $G$ em $d$ tem somente uma componente conexa.

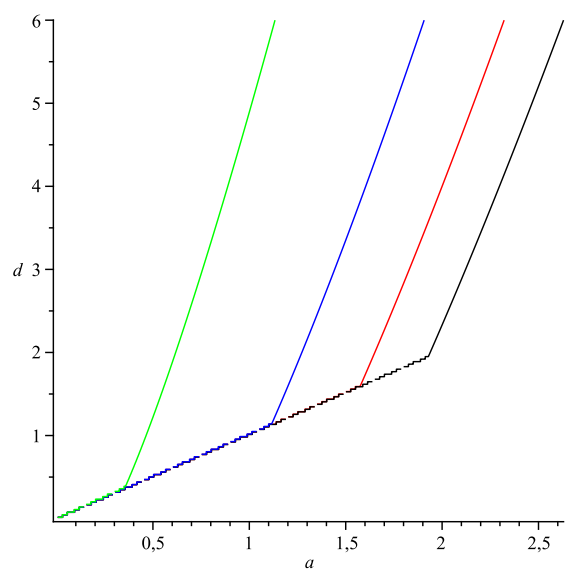

figura 21: Gráfico de $\partial G / \partial d=0$ para diferentes valores de $b c$

Como o gráfico da derivada de $G$ está inteiramente contido no primeiro quadrante, e sendo que $G(a, b, c, d)=0$ possui uma parte conexa para $d<0$, sempre podemos considerar essa parte conexa pois aí a derivada de $G$ não se anula. Isso confirma a afirmação acima. 
A conclusão obtida da afirmação que acabamos de provar é que a variável $d$ pode ser considerada como sendo um parâmetro do campo regularizado. Portanto, iremos, de agora em diante, considerar em primeiro lugar que $d$ é negativo, e em segundo que $d$ é o segundo parâmetro do campo regularizado.

Lema 23. Sejam $Z_{\lambda}$ uma $F C D$ do tipo $\mathcal{F}_{4}$ tal que $a^{2}+b c \neq 0, \varepsilon>0$ e $\varphi$ a função de transição. Se $d=\frac{\left(a^{2}+b c\right) \beta}{\alpha c}-a \neq 0$ então existe um único $\lambda$ para o qual o campo regularizado, $Z_{\lambda, R}$, possui uma única singularidade que anula o traço e o determinante da matriz Jacobiana.

Demonstração. A família de campos regularizados é dada por

$$
\begin{aligned}
& Z_{\lambda, \varepsilon}(x, y)=\left(\left(\frac{1}{2}-\frac{y}{2 \sqrt{y^{2}+\varepsilon^{2}}}\right) \alpha+\left(\frac{1}{2}+\frac{y}{2 \sqrt{y^{2}+\varepsilon^{2}}}\right)(a x+b y+e \lambda),\right. \\
& \left.\left(\frac{1}{2}-\frac{y}{2 \sqrt{y^{2}+\varepsilon^{2}}}\right) \beta+\left(\frac{1}{2}+\frac{y}{2 \sqrt{y^{2}+\varepsilon^{2}}}\right)(c x+d y+f \lambda)\right) .
\end{aligned}
$$

Aqui podemos efetuar a mudança de variáveis e reescalonamento dos parâmetros conforme o que fizemos para chegar na expressão (3.12) que define $Z_{\lambda, R}$.

A única singularidade, $p_{B T}=\left(x_{B T}, y_{B T}, d_{B T}, \lambda_{B T}\right)$, onde o traço e o determinante de $A=D Z_{\lambda, R}(x, y)$ se anulam é dada por

$$
\begin{aligned}
& x_{B T}=-\frac{f_{1} W^{2}+f_{2} W+f_{3}}{16 \alpha^{2} c^{2}(a f-c e)\left(f_{4} W^{2}+f_{5} W+f_{6}\right)} \\
& y_{B T}=-\frac{a^{2}+b c}{8 \alpha c}+\frac{\alpha c}{2 W^{2}\left(a^{2}+b c\right)} \\
& d_{B T}=\frac{\left(a^{2}+b c\right) \beta}{\alpha c}-a \\
& \lambda_{B T}=\frac{\left(a^{2}+b c\right)(-\alpha c+a \beta)\left(\left(a^{2}+b c\right)\left(-W\left(a^{2}+b c\right)+8 W^{2} c \alpha-2 \alpha c\right)-12 W \alpha^{2} c^{2}\right)}{8 c^{3} \alpha^{3}(a f-e c)}
\end{aligned}
$$

onde

$$
\begin{aligned}
& W=\frac{\left(V\left(a^{2}+b c\right)^{2}\right)^{1 / 3}}{24 c \alpha\left(a^{2}+b c\right)}+\frac{\left(96 c^{2} \alpha^{2}+\left(a^{2}+b c\right)^{2}\right)\left(a^{2}+b c\right)}{24 c \alpha\left(V\left(a^{2}+b c\right)^{2}\right)^{1 / 3}}+\frac{a^{2}+b c}{24 c \alpha}, \\
& V=\left(a^{2}+b c\right)^{4}+144 c^{2} \alpha^{2}\left(\left(a^{2}+b c\right)^{2}+24 c^{2} \alpha^{2}\right)+192 c^{3} \alpha^{3} \sqrt{3\left(a^{2}+b c\right)^{2}+324 c^{2} \alpha^{2}}
\end{aligned}
$$




$$
\begin{aligned}
& f_{1}=e \beta\left(a^{2}+b c\right)^{8}+\alpha\left(a^{2}+b c\right)^{7}(a(a f-c e)-c(a e+b f))+64 c^{2} e \alpha^{2} \beta\left(a^{2}+b c\right)^{6} \\
& +64 c^{2} \alpha^{3}\left(a^{2}+b c\right)^{5}(2 a(a f-c e)-c(a e+b f))+1280 c^{4} \alpha^{4}\left(a^{2}+b c\right)^{4}(f \alpha-e \beta)+2560 a c^{4} \alpha^{5} \\
& \left(a^{2}+b c\right)^{3}(a f-c e)+16384 c^{6} \alpha^{6}\left(a^{2}+b c\right)^{2}(f \alpha-2 e \beta)+16384 c^{7} \alpha^{7}\left(a^{2}+b c\right)(a e+b f) \\
& f_{2}=4 c e \alpha \beta\left(a^{2}+b c\right)^{7}+4 c \alpha^{2}\left(a^{2}+b c\right)^{6}(a(a f-c e)-c(a e+b f))+160 c^{3} e \alpha^{3} \beta\left(a^{2}+b c\right)^{5} \\
& +32 c^{3} \alpha^{4}\left(a^{2}+b c\right)^{4}(13 a(a f-c e)-5 c(a e+b f))+6144 c^{5} \alpha^{5}\left(a^{2}+b c\right)^{3}(f \alpha-e \beta)+2048 a c^{5} \alpha^{6}\left(a^{2}\right. \\
& +b c)^{2}(a f-c e)-24576 c^{7} e \alpha^{7} \beta\left(a^{2}+b c\right)+8192 c^{7} \alpha^{8}(a(a f+c e)+c(a e+3 b f)) \\
& f_{3}=4 c^{2} e \alpha^{2} \beta\left(a^{2}+b c\right)^{6}+4 c^{2} \alpha^{3}\left(a^{2}+b c\right)^{5}(a(a f-c e)-c(a e+b f))+128 c^{4} e \alpha^{4} \beta\left(a^{2}+b c\right)^{4} \\
& +128 c^{4} \alpha^{5}\left(a^{2}+b c\right)^{3}(3 a(a f-c e)-c(a e+b f))+6144 c^{6} \alpha^{6}\left(a^{2}+b c\right)^{2}(f \alpha-e \beta) \\
& f_{4}=\left(a^{2}+b c\right)^{2}\left(\left(a^{2}+b c\right)^{4}+96 c^{2} \alpha^{2}\left(\left(a^{2}+b c\right)^{2}+16 c^{2} \alpha^{2}\right)\right) \\
& f_{5}=4 c \alpha\left(a^{2}+b c\right)\left(\left(a^{2}+b c\right)^{2}+8 c^{2} \alpha^{2}\right)\left(\left(a^{2}+b c\right)^{2}+64 c^{2} \alpha^{2}\right) \\
& f_{6}=4 c^{2} \alpha^{2}\left(\left(a^{2}+b c\right)^{4}+64 c^{2} \alpha^{2}\left(\left(a^{2}+b c\right)^{2}+4 c^{2} \alpha^{2}\right)\right)
\end{aligned}
$$

Como $\alpha \neq 0$, ce $-a f<0, c<0$, as expressões do ponto $p_{B T}$ fazem sentido se supormos que $a^{2}+b c \neq 0$. De fato, se $a^{2}+b c \neq 0$ então a função $V$ não se anula. Além disso, a função $W$ se anula para um determinado valor complexo de $V$, e também o seu conjugado, como não estamos interessados em valores complexos, segue que $W \neq 0$. Agora vamos verificar que o denominador de $x_{B T}$ não se anula. Para isso, substituimos os valores de $f_{4}, f_{5}$ e $f_{6}$ no discriminante da solução do polinômio quadrático em $W$. Substituindo $a^{2}+b c$ por $u$ e $c \alpha$ por $v$ no valor do discriminante obtemos que para quaisquer valores de $u \neq 0$ e $v<0$ este discriminante é negativo, ou seja, o denominador de $x_{B T}$ não se anula em $\mathbb{R}$.

A função $V$ é estritamente positiva para quaisquer valores de $\alpha c<0$ e $a^{2}+b c \in \mathbb{R}^{*}$. A função $W$ será estritamente positiva se $a^{2}+b c<0$ e estritamente negativa caso contrário.

Como o parâmetro $d$ é negativo, segue que a interseção de $G(a, b, c, d)=0$ com $d_{B T}$ dado em (3.41) se dá, conforme a figura seguinte, em um único ponto. 


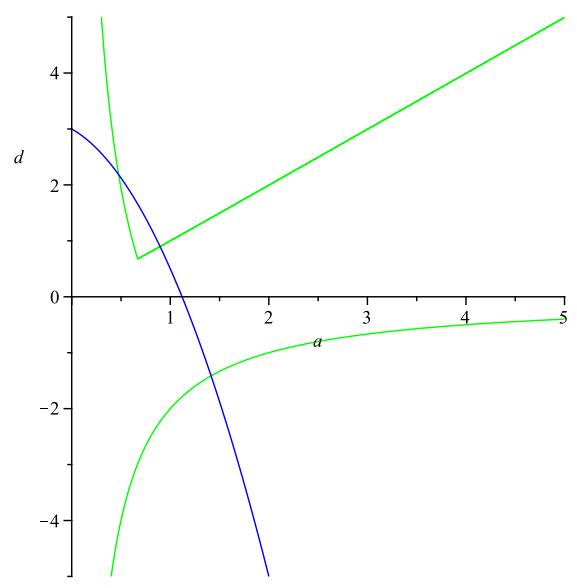

figura 22: Gráficos de $d$ e $G(a, b, c, d)=0$

Obs.: O fato de termos considerado $d_{B T} \neq 0$ no lema anterior, nos leva a $a^{2}+b c \neq a c \alpha / \beta$, ao passo que se tivéssemos $a^{2}+b c=a c \alpha / \beta$, então não teríamos um candidato a $d_{B T}$.

Para simplificar os resultados abaixo vamos analisar as coordenadas, e as funções envolvidas, do ponto $p_{B T}$ e dos dois elementos da primeira linha da matriz Jacobiana da família regularizada, deixando em evidência a variável $\beta$. Assim, reescrevendo as funções $f_{1}, f_{2}$ e $f_{3}$, definidas após (3.41), em termos de $\beta$ obtemos

$$
\begin{aligned}
& f_{1}=F_{1}(a, b, c, e, f, \alpha) \beta+F_{2}(a, b, c, e, f, \alpha) \\
& f_{2}=F_{3}(a, b, c, e, f, \alpha) \beta+F_{4}(a, b, c, e, f, \alpha) \\
& f_{3}=F_{5}(a, b, c, e, f, \alpha) \beta+F_{6}(a, b, c, e, f, \alpha),
\end{aligned}
$$


onde ${ }^{2}$

$$
\begin{aligned}
F_{1} & =e\left(a^{2}+b c\right)^{8}+64 c^{2} e \alpha^{2}\left(a^{2}+b c\right)^{6}-1280 c^{4} e \alpha^{4}\left(a^{2}+b c\right)^{4}-32768 c^{6} e \alpha^{6}\left(a^{2}+b c\right)^{2} \\
F_{2} & =\alpha\left(a^{2}+b c\right)^{7}(a(a f-c e)-c(a e+b f))+64 c^{2} \alpha^{3}\left(a^{2}+b c\right)^{5}(2 a(a f-c e)-c(a e+b f)) \\
& +1280 c^{4} f \alpha^{5}\left(a^{2}+b c\right)^{4}+2560 a c^{4} \alpha^{5}\left(a^{2}+b c\right)^{3}(a f-c e)+16384 c^{6} f \alpha^{7}\left(a^{2}+b c\right)^{2} \\
& +16384 c^{7} \alpha^{7}\left(a^{2}+b c\right)(a e+b f), \\
F_{3} & =4 c e \alpha\left(a^{2}+b c\right)^{7}+160 c^{3} e \alpha^{3}\left(a^{2}+b c\right)^{5}-6144 c^{5} e \alpha^{5}\left(a^{2}+b c\right)^{3}-24576 c^{7} e \alpha^{7}\left(a^{2}+b c\right) \\
F_{4} & =4 c \alpha^{2}\left(a^{2}+b c\right)^{6}(a(a f-c e)-c(a e+b f))+32 c^{3} \alpha^{4}\left(a^{2}+b c\right)^{4}(13 a(a f-c e)-5 c(a e+b f)) \\
& +6144 c^{5} f \alpha^{6}\left(a^{2}+b c\right)^{3}+2048 a c^{5} \alpha^{6}\left(a^{2}+b c\right)^{2}(a f-c e)+8192 c^{7} \alpha^{8}(a(a f+c e)+c(a e+3 b f)), \\
F_{5} & =4 c^{2} e \alpha^{2}\left(a^{2}+b c\right)^{6}+128 c^{4} e \alpha^{4}\left(a^{2}+b c\right)^{4}-6144 c^{6} e \alpha^{6}\left(a^{2}+b c\right)^{2} \\
F_{6} & =4 c^{2} \alpha^{3}\left(a^{2}+b c\right)^{5}(a(a f-c e)-c(a e+b f))+128 c^{4} \alpha^{5}\left(a^{2}+b c\right)^{3}(3 a(a f-c e)-c(a e+b f)) \\
& +6144 c^{6} f \alpha^{7}\left(a^{2}+b c\right)^{2} .
\end{aligned}
$$

Substituindo as expressões $F_{i}, 1 \leq i \leq 6$, na fórmula que dá $x_{B T}$ e notando que $f_{4}, f_{5}$, $f_{6}$ e $W$ não dependem de $\beta$ escrevemos

$$
x_{B T}=F_{7} \beta+F_{8}
$$

onde

$$
\begin{aligned}
& F_{7}=-\frac{F_{1} W^{2}+F_{3} W+F_{5}}{16 c^{2} \alpha^{2}(a f-c e)\left(f_{4} W^{2}+f_{5} W+f_{6}\right)} \\
& F_{8}=-\frac{F_{2} W^{2}+F_{4} W+F_{6}}{16 c^{2} \alpha^{2}(a f-c e)\left(f_{4} W^{2}+f_{5} W+f_{6}\right)} .
\end{aligned}
$$

A coordenada $y_{B T}$ de $p_{B T}$ não depende de $\beta$. Portanto, vamos denotá-la por $F_{9}$ onde

$$
y_{B T}=F_{9}=-\frac{a^{2}+b c}{8 \alpha c}+\frac{\alpha c}{2 W^{2}\left(a^{2}+b c\right)} .
$$

A coordenada $d_{B T}$ será reescrita como

$$
d_{B T}=F_{10} \beta-a
$$

onde

$F_{10}=\frac{a^{2}+b c}{\alpha c}$

\footnotetext{
${ }^{2}$ Para uma melhor visualização dos resultados, de agora em diante iremos escrever apenas $F_{i}$ para as funções descritas abaixo.
} 
A coordenada $\lambda_{B T}$ será reescrita como

$$
\lambda_{B T}=F_{11} \beta+F_{12}
$$

onde

$F_{11}=\frac{a\left(a^{2}+b c\right)\left(\left(a^{2}+b c\right)\left(-W\left(a^{2}+b c\right)+8 W^{2} c \alpha-2 \alpha c\right)-12 W \alpha^{2} c^{2}\right)}{8 c^{3} \alpha^{3}(a f-e c)}$
$F_{12}=\frac{-c \alpha\left(a^{2}+b c\right)\left(\left(a^{2}+b c\right)\left(-W\left(a^{2}+b c\right)+8 W^{2} c \alpha-2 \alpha c\right)-12 W \alpha^{2} c^{2}\right)}{8 c^{3} \alpha^{3}(a f-e c)}$.

O primeiro elemento da primeira linha da matriz Jacobiana, $Z_{x}^{1}$, calculado no ponto $p_{B T}$, não depende de $\beta$ donde iremos denotá-lo por $F_{13}$ onde

$F_{13}=\frac{a\left(y_{B T}+\sqrt{1+y_{B T}^{2}}\right)}{2 \sqrt{1+y_{B T}^{2}}}$.

O segundo elemento da primeira linha do jacobiano, $Z_{y}^{1}$, calculado no ponto $p_{B T}$ será reescrito como

$$
Z_{y}^{1}=F_{14} \beta+F_{15}
$$

onde

$$
\begin{aligned}
& F_{14}=\frac{a F_{7}+e F_{11}}{2\left(1+\left(F_{9}\right)^{2}\right)^{3 / 2}} \\
& F_{15}=\frac{a F_{8}-\alpha+e F_{12}+2 b F_{9}+b\left(F_{9}\right)^{3}+b\left(1+\left(F_{9}\right)^{2}\right)^{3 / 2}}{2\left(1+\left(F_{9}\right)^{2}\right)^{3 / 2}} .
\end{aligned}
$$

Os próximos quatro lemas verificam as condições do teorema de Bogdanov-Takens.

Lema 24. Seja $Z_{\lambda, R}$ a FCR do lema anterior. Então, o item BT.0) do Teorema 22 está satisfeito.

Demonstração. Vamos verificar que a matriz $A$ aplicada no ponto $\left(x_{B T}, y_{B T}, d_{B T}, \lambda_{B T}\right)$ é não-nula. De fato, observando o elemento $a_{11}$ temos que

$$
a_{11}=\left(\frac{1}{2}+\frac{y_{B T}}{2 \sqrt{1+y_{B T}^{2}}}\right) a
$$

que nada mais é do que a função de transição calculada em $y_{B T}$. Mas, como a função de transição $\varphi(y)$ nunca se anula e $a \neq 0$, concluímos que o elemento $a_{11}$ de $A_{B T}=$ $D Z_{\lambda, R}\left(x_{B T}, y_{B T}, d_{B T}, \lambda_{B T}\right)$ é diferente de zero, implicando que $A \neq 0$. 
Para os próximos lemas vamos precisar calcular os autovetores, autovetores generalizados e os termos $\xi_{i}, i=1,2,3$ que apresentamos a seguir.

Em primeiro lugar, vamos calcular o autovetor, $q_{0}$, de $A_{B T}$ correspondente ao autovalor zero, e também o autovetor generalizado $q_{1}$ de $A_{B T}$ correspondente ao autovetor $q_{0}$, ou seja, $A_{B T} q_{1}=q_{0}$. Do mesmo modo, calcularemos o autovetor $p_{1}$ e o autovetor generalizado $p_{0}$ ambos da matriz transposta $A^{T}$.

Vamos denotar os elementos da matriz Jacobiana da família regularizada aplicada no ponto $p_{B T}$ simplesmente por

$$
A_{B T}=\left(\begin{array}{cc}
Z_{x}^{1} & Z_{y}^{1} \\
Z_{x}^{2} & Z_{y}^{2}
\end{array}\right) .
$$

Como o traço e o determinante dessa matriz são nulos então $Z_{x}^{1}=-Z_{y}^{2}$ e substituindo essa igualdade na expressão do determinante temos que $-\left(Z_{x}^{1}\right)^{2}-Z_{y}^{1} Z_{x}^{2}=0$, e como $Z_{x}^{1} \neq 0$, por (3.42), concluímos que $Z_{y}^{1}$ e $Z_{x}^{2}$ são ambos não-nulos. Assim, podemos reescrever a matriz $A_{B T}$ como

$$
A_{B T}=\left(\begin{array}{cc}
Z_{x}^{1} & Z_{y}^{1} \\
-\left(Z_{x}^{1}\right)^{2} / Z_{y}^{1} & -Z_{x}^{1}
\end{array}\right) .
$$

Calculando os vetores $p_{0}, p_{1}, q_{0}$ e $q_{1}$ que satisfazem as equações $A_{B T} q_{0}=0, A_{B T} q_{1}=q_{0}$, $A_{B T}^{T} p_{1}=0, A_{B T}^{T} p_{1}=p_{0},<p_{0}, q_{0}>=1$ e $<p_{1}, q_{1}>=1$ e usando o método de GrammSchmidt para que tenhamos $<p_{0}, q_{1}>=0$ e $<p_{1}, q_{0}>=0$ obtemos

$$
\begin{aligned}
& q_{0}=\left(1,-\frac{Z_{x}^{1}}{Z_{y}^{1}}\right) \\
& q_{1}=\left(\frac{1-Z_{y}^{1}}{Z_{x}^{1}}, 1\right) \\
& p_{0}=\left(\frac{(k-1)\left(Z_{y}^{1}\right)^{2}+k\left(Z_{x}^{1}\right)^{2}-(k-1) Z_{y}^{1}}{\left(Z_{x}^{1}\right)^{2}+\left(Z_{y}^{1}\right)^{2}-2 Z_{y}^{1}+1}, \frac{\left(Z_{y}^{1}-1\right)\left((k-1)\left(Z_{y}^{1}\right)^{2}+k\left(Z_{x}^{1}\right)^{2}-(k-1) Z_{y}^{1}\right)}{Z_{x}^{1}\left(\left(Z_{x}^{1}\right)^{2}+\left(Z_{y}^{1}\right)^{2}-2 Z_{y}^{1}+1\right)}\right) \\
& p_{1}=\left(Z_{x}^{1}, Z_{y}^{1}\right)
\end{aligned}
$$

onde $k \neq 1$ é uma constante pré-fixada.

Agora que temos os vetores, vamos calcular os valores de $\xi_{1}$ e $\xi_{2}$ e verificar que são diferentes de zero.

Vamos denotar as coordenadas de $q_{0}$ por $\left(x_{1}, x_{2}\right)$, o ponto $p_{B T}=\left(x_{B T}, y_{B T}\right)$ e o campo 
$Z_{\lambda, R}=\left(Z^{1}, Z^{2}\right)$. Assim o termo $B\left(q_{0}, q_{0}\right)$ é dado por

$$
\begin{aligned}
& B\left(q_{0}, q_{0}\right)=\left(\frac{\partial^{2} Z^{1}}{\partial x^{2}}\left(p_{B T}\right) x_{1}^{2}+2 \frac{\partial^{2} Z^{1}}{\partial x \partial y}\left(p_{B T}\right) x_{1} x_{2}+\frac{\partial^{2} Z^{1}}{\partial y^{2}}\left(p_{B T}\right) x_{2}^{2},\right. \\
& \left.\frac{\partial^{2} Z^{2}}{\partial x^{2}}\left(p_{B T}\right) x_{1}^{2}+2 \frac{\partial^{2} Z^{2}}{\partial x \partial y}\left(p_{B T}\right) x_{1} x_{2}+\frac{\partial^{2} Z^{2}}{\partial y^{2}}\left(p_{B T}\right) x_{2}^{2}\right) \\
& =\left(-\frac{Z_{x}^{1}\left(2 a y^{2} Z_{y}^{1}+2 a Z_{y}^{1}-3 \alpha y Z_{x}^{1}+3 a y x Z_{x}^{1}+b y^{2} Z_{x}^{1}+3 e \lambda y Z_{x}^{1}-2 b Z_{x}^{1}\right)}{2\left(y^{2}+1\right)^{5 / 2}\left(Z_{y}^{1}\right)^{2}},\right. \\
& \left.-\frac{Z_{x}^{1}\left(2 c y^{2} Z_{y}^{1}+2 c Z_{y}^{1}-3 \beta y Z_{x}^{1}+3 c y x Z_{x}^{1}+d y^{2} Z_{x}^{1}+3 f \lambda y Z_{x}^{1}-2 d Z_{x}^{1}\right)}{2\left(y^{2}+1\right)^{5 / 2}\left(Z_{y}^{1}\right)^{2}}\right)
\end{aligned}
$$

substituindo na fórmula $\xi_{1}=\frac{<p 1, B\left(q_{0}, q_{0}\right)>}{2}$ obtemos

$$
\begin{aligned}
\xi_{1} & =\frac{1}{4\left(Z_{y}^{1}\right)^{2}\left(1+y^{2}\right)^{5 / 2}}\left(\left[3 y(\alpha-a x-e \lambda)+b\left(2-y^{2}\right)\right]\left(Z_{x}^{1}\right)^{3}+[3 y(\beta-f \lambda-c x)\right. \\
& \left.\left.-y^{2}(2 a+d)+2 d-2 a\right]\left(Z_{x}^{1}\right)^{2} Z_{y}^{1}-2 c\left(1+y^{2}\right) Z_{x}^{1}\left(Z_{y}^{1}\right)^{2}\right) .
\end{aligned}
$$

Substituindo as expressões de $Z_{x}^{1}, Z_{y}^{1}, x_{B T}, y_{B T}, d_{B T}$ e $\lambda_{B T}$, dados na prova do Lema 23, em (3.45) obtemos que $\xi_{1}$ é um polinômio de segundo grau em $\beta$ dado por

$$
P(\beta)=\zeta_{1} \beta^{2}+\zeta_{2} \beta+\zeta_{3}
$$

onde

$$
\begin{aligned}
\zeta_{1} & =\left(2 F_{10}+3 F_{9}\left(1-c F_{7}-f F_{11}\right)-F_{10} F_{9}^{2}\right) F_{13}^{2} F_{14}+F_{13} F_{14}^{2} \\
\zeta_{2} & =-3\left(a F_{7}+e F_{11}\right) F_{9} F_{13}^{3}+\left(3 F_{9}\left(-c F_{8}-f F_{12}\right)+a F_{9}^{2}-2 a\left(F_{9}^{2}+1\right)-2 a\right) F_{13}^{2} F_{14}+\left(2 F_{10}\right. \\
& \left.+3 F_{9}\left(1-c F_{7}-f F_{11}\right)-F_{10} F_{9}^{2}\right) F_{13}^{2} F_{15}+2 F_{13} F_{15} F_{14} \\
\zeta_{3} & =-3 F_{9}\left(a F_{8}+e F_{12}\right) F_{13}^{3}+F_{12}+\left(3 F_{9}\left(-c F_{8}-f F_{12}\right)+a F_{9}^{2}-2 a\left(F_{9}^{2}+1\right)-2 a\right) F_{13}^{2} F_{15} \\
& +F_{13} F_{15}^{2} .
\end{aligned}
$$

Notamos que $\zeta_{1}$ é um polinômio de segundo grau em $F_{13}$ sem termo constante. Logo, $\zeta_{1}$ 
tem uma raiz em $F_{13}=0$ e outra quando $F_{13}$ for igual a

$$
r_{13}=\frac{F_{14}}{-2 F_{10}-3 F_{9}\left(1-c F_{7}-f F_{11}\right)+F_{10} F_{9}^{2}} .
$$

Porém, notamos que $F_{13}=0$ nunca ocorre pois $F_{13}$ nunca se anula, e é sempre positivo.

Agora, vamos verificar quando a segunda raiz de $\zeta_{1}$ está definida. O denominador de (3.47) se anula quando $F_{7}$ for igual a

$$
r_{7}=\frac{2 F_{10}+3 F_{9}-3 f F_{9} F_{11}+F_{9}^{2} F_{10}}{3 c F_{9}} .
$$

Logo, para que a expressão $F_{13}=r_{13}$ faça sentido devemos supor que $F_{7} \neq r_{7}$. Substituindo os valores de $F_{7}, F_{9}$ e $F_{11}$ na igualdade $F_{7}=r_{7}$ obtemos que af $-c e$ deve ser distinto de

$$
\pm \frac{W}{4} \sqrt{\frac{-3 a F_{10}\left(W^{2} F_{10}^{2}-4\right)\left(8 W^{2} F_{10}-W F_{10}^{2}-12 W-2 F_{10}\right)\left(F_{1} W^{2}+F_{3} W+F_{5}\right)}{\left(-104 W^{4} F_{10}^{2}-96 W^{2}+W^{4} F_{10}^{4}-8 W^{2} F_{10}^{2}+16\right)\left(f_{4} W^{2}+f_{5} W+f_{6}\right) c \alpha}}
$$

quando o radicando fizer sentido, ou seja, for positivo. Notamos ainda que $W, f_{4}, f_{5}, f_{6}$ e $F_{10}$ dependem apenas dos termos $c \alpha$ e $a^{2}+b c$. Por outro lado, fazendo $F_{1}=e \widetilde{F}_{1}, F_{3}=e \widetilde{F}_{3}$ e $F_{5}=e \widetilde{F}_{5}$, obtemos que $\widetilde{F}_{i}, i=1,3,5$, é em função de $c \alpha$ e $a^{2}+b c$. Portanto, se $e>0$ e

$$
\frac{a f-c e}{\sqrt{a e}} \neq \widetilde{W}_{ \pm}
$$

onde $\widetilde{W}_{ \pm}$são dados, respectivamente, por

$$
\pm \frac{W}{4} \sqrt{\frac{-3 a F_{10}\left(W^{2} F_{10}^{2}-4\right)\left(8 W^{2} F_{10}-W F_{10}^{2}-12 W-2 F_{10}\right)\left(\widetilde{F}_{1} W^{2}+\widetilde{F}_{3} W+\widetilde{F}_{5}\right)}{\left(-104 W^{4} F_{10}^{2}-96 W^{2}+W^{4} F_{10}^{4}-8 W^{2} F_{10}^{2}+16\right)\left(f_{4} W^{2}+f_{5} W+f_{6}\right) c \alpha}}
$$

então $F_{7} \neq r_{7}$, implicando que $F_{13}=r_{13}$ está bem definido.

Analisando $\widetilde{W}_{+}$e considerando as variáveis $u=c \alpha$ e $v=a^{2}+b c$ obtemos que o seu limite quando $v \rightarrow \pm \infty$ vale $\mp \infty$. Fixando um valor para $v$ observamos que o gráfico de $\widetilde{W}_{+}$, no plano $(v, \mathbb{R})$, é simétrico com relação a origem. Considerando $v>0$ notamos que o gráfico é desconexo, existem duas componentes conexas sendo que uma delas está localizada em um intervalo $I_{1}=\left(v_{1}, v_{2}\right)$ próximo a $v=0$ e a outra em um intervalo $I_{2}=\left(v_{3}, \infty\right)$. Considerando $u=-0,5$ obtemos que $v_{1} \approx 0,223944, v_{2} \approx 2,607643, v_{3} \approx 5,09924$. Além disso, o limite de $\widetilde{W}_{+}$quando $v$ tende a $v_{1}$ e $v_{3}$ é $-\infty$, ao passo que quando $v$ tende a $v_{2}$ o limite vale zero. A 
próxima figura contém o gráfico de $\widetilde{W}_{+}$nesses intervalos, o gráfico da direita é referente ao intervalo $I_{2}$ e o da esquerda ao intervalo $I_{1}$, para o caso particular $u=-0,5$, considerando o semi-plano $v>0$.
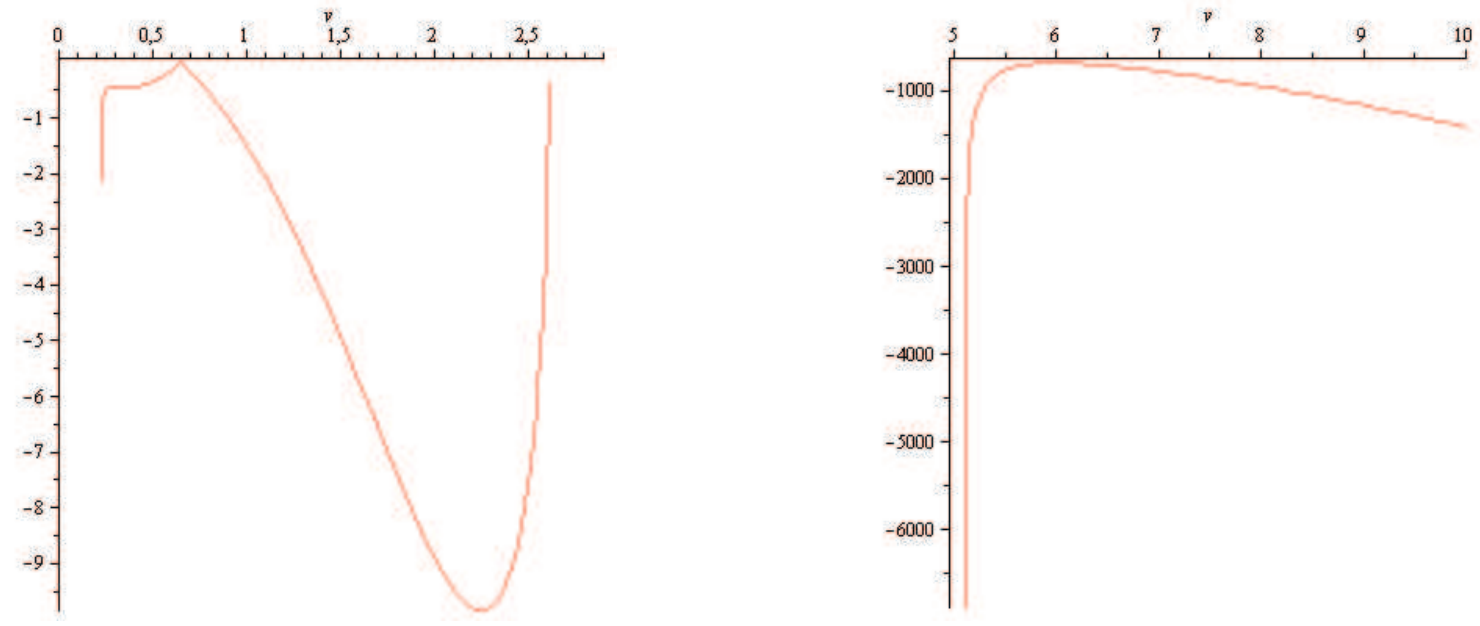

figura 23: Gráfico de $\widetilde{W}_{+}$nos intervalos $I_{1}$ e $I_{2}$

O gráfico de $\widetilde{W}_{+}$para $v<0$ está contido no quarto quadrante do plando $(v, \mathbb{R})$ e tem as propriedades opostas, com relação ao sinal, do gráfico apresentado na figura acima.

Assim, como af $-c e>0$ temos que (3.50) sempre é satisfeito quando consideramos $\widetilde{W}_{+}<0$ e $a^{2}+b c>0$. Se $a^{2}+b c<0$, ainda para $\widetilde{W}_{+}$, então a desigualdade (3.50) não será satisfeita em apenas dois valores de $v$, que são as pré-imagens por $\widetilde{W}_{+}$de $\frac{a f-c e}{\sqrt{a e}}$. Porém, evitaremos apresentar esses pontos pela sua complexidade e tamanho.

A análise da expressão de $\widetilde{W}_{-}$é análoga à anterior, sendo que para $v<0$ não ocorre problemas e se $v>0$ então apenas dois valores de $v$ não podem ser considerados.

Resumindo, para que $r_{13}$ esteja bem definido devemos considerar que

$$
\begin{aligned}
& \widetilde{W}_{+}<0, \text { se } a^{2}+b c>0 \\
& \widetilde{W}_{-}>0, \text { se } a^{2}+b c<0 .
\end{aligned}
$$

Supondo que $r_{13}$ está bem definida, vamos verificar quando $F_{13} \neq r_{13}$. Substituindo os valores de $F_{14}$ e logo após os de $F_{7}$ e $F_{11}$ na expressão de $r_{13}$, e escrevendo $F_{13}$ em função de 
$F_{9}$ obtemos que $F_{13}=r_{13}$ se reduz a

$a\left(F_{9}+\sqrt{1+F_{9}^{2}}\right)=\frac{e\left(a P_{1} Q_{2}+P_{2} Q_{1}\right)}{\left(1+F_{9}^{2}\right)\left(\left(-2 F_{10}-3 F_{9}+F_{10} F_{9}^{2}\right)(a f-c e) Q_{1} Q_{2}+3 F_{9}\left(c e P_{1} Q_{2}+f Q_{1} P_{2}\right)\right)}$

onde, denotamos $\widetilde{F}_{i}=\frac{F_{i}}{e}$ para $i=1,3,5$,

$$
\begin{aligned}
P_{1} & =\widetilde{F}_{1} W^{2}+\widetilde{F}_{3} W+\widetilde{F}_{5} \\
P_{2} & =a c \alpha\left(c \alpha\left(-W\left(a^{2}+b c\right)+8 W^{2} c \alpha-2 c \alpha\right)-12 W(c \alpha)^{2}\right) \\
Q_{1} & =-16(c \alpha)^{2}\left(f_{4} W^{2}+f_{5} W+f_{6}\right) \\
Q_{2} & =8(c \alpha)^{3} .
\end{aligned}
$$

A única solução de (3.53) é quando $f=f_{s}$ onde $f_{s}$ é dada por

$$
\frac{\left.e\left(P_{2} Q_{1}+a P_{1} Q_{2}+a c Q_{2}\left(1+F_{9}^{2}\right)\left(F_{9}+\left(1+F_{9}^{2}\right)^{1 / 2}\right)\left[-2 F_{10} Q_{1}-3 F_{9} Q_{1}-3 F_{9} P_{1}+F_{10} F_{9}^{2} Q_{1}\right]\right)\right)}{a Q_{1}\left(\left(1+F_{9}^{2}\right)\left(F_{9}+\left(1+F_{9}^{2}\right)^{1 / 2}\right)\left(a F_{10} F_{9}^{2} Q_{2}-2 a F_{10} Q_{2}-3 a F_{9} Q_{2}+3 F_{9} P_{2}\right)\right)} .
$$

A quantidade $\frac{f_{s}}{e}$ está em função somente de $a, b, c, \alpha$. Portanto, temos que se $\frac{f}{e} \neq f_{s}$ então $\zeta_{1} \neq 0$.

Do que foi visto acima provamos o seguinte lema.

Lema 25. Seja $Z_{\lambda, R}$ a FCR do Lema 23. Se e $>0, \frac{f}{e} \neq f_{s}$, onde $f_{s}$ é dado por (3.54), $\widetilde{W}_{+}<0$ quando $a^{2}+b c>0$ e $\widetilde{W}_{-}>0$ quando $a^{2}+b c<0$, onde $\widetilde{W}_{ \pm}$são dados por (3.51), então para valores de $\beta$ distintos das raízes de $P(\beta)$ vale o item BT.1) do Teorema 22.

Agora, vamos calcular

$$
\xi_{2}=<p_{0}, B\left(q_{0}, q_{0}\right)>+<p_{1}, B\left(q_{0}, q_{1}\right)>
$$

O termo $B\left(q_{0}, q_{1}\right)$, onde $q_{0}=\left(x_{1}, x_{2}\right)$ e $q_{1}=\left(y_{1}, y_{2}\right)$, é dado por 


$$
\begin{aligned}
& B\left(q_{0}, q_{1}\right)=\left(\frac{\partial^{2} Z^{1}}{\partial x^{2}}\left(p_{B T}\right) x_{1} y_{1}+\frac{\partial^{2} Z^{1}}{\partial x \partial y}\left(p_{B T}\right) x_{1} y_{2}+\frac{\partial^{2} Z^{1}}{\partial y \partial x}\left(p_{B T}\right) x_{2} y_{1}+\frac{\partial^{2} Z^{1}}{\partial y^{2}}\left(p_{B T}\right) x_{2} y_{2}\right. \\
& \left.\frac{\partial^{2} Z^{2}}{\partial x^{2}}\left(p_{B T}\right) x_{1} y_{1}+\frac{\partial^{2} Z^{2}}{\partial x \partial y}\left(p_{B T}\right) x_{1} y_{2}+\frac{\partial^{2} Z^{2}}{\partial y \partial x}\left(p_{B T}\right) x_{2} y_{1}+\frac{\partial^{2} Z^{2}}{\partial y^{2}}\left(p_{B T}\right) x_{2} y_{2}\right) \\
& =\left(\frac{\left(-3 y(\alpha-a x+e \lambda)+b y^{2}-2 b\right) Z_{x}^{1}-a\left(1+y^{2}\right)+2 a\left(1+y^{2}\right) Z_{y}^{1}}{2\left(1+y^{2}\right)^{5 / 2} Z_{y}^{1}}\right. \\
& \left.\frac{\left(3 y(-\beta+c x+f \lambda)+d y^{2}-2 d\right) Z_{x}^{1}-c\left(1+y^{2}\right)+2 c\left(1+y^{2}\right) Z_{y}^{1}}{2\left(1+y^{2}\right)^{5 / 2} Z_{y}^{1}}\right)
\end{aligned}
$$

Efetuando o produto vetorial dos vetores envolvidos temos finalmente que

$$
\xi_{2}=z_{0}+z_{1} \beta+z_{2} \beta^{2}+z_{3} \beta^{3}+z_{4} \beta^{4}
$$

onde

$$
\begin{aligned}
z_{0} & =\left[b F_{13}^{4}+\left(a F_{15}-a\right) F_{13}^{3}+\left((b+2 c) F_{15}^{2}-(b+c) F_{15}\right) F_{13}^{2}+a\left(-2-2 F_{15}^{2}+F_{15}^{3}+3 F_{15}\right) F_{13}\right. \\
& \left.+2 c F_{15}^{4}+c F_{15}-3 c F_{15}^{3}\right] F_{9}^{2}+\left[3\left(a F_{8}+e F_{12}-\alpha\right)\left(F_{13}^{2}+F_{15}^{2}-F_{15}\right) F_{13}^{2}+3\left(f F_{12}\right.\right. \\
& \left.\left.+c F_{8}\right)\left(F_{15} F_{13}^{2}+F_{13}^{3}-F_{15}^{2}-F_{15}+1\right) F_{13}\right] F_{9}-2 b F_{13}^{4}+a\left(-1+4 F_{15}\right) F_{13}^{3}+\left(2(-b+c) F_{15}^{2}\right. \\
& \left.+(2 b-c) F_{15}\right) F_{13}^{2}+a\left(4 F_{15}^{3}-5 F_{15}^{2}+1\right) F_{13}+2 c F_{15}^{4}+c F_{15}-3 c F_{15}^{3}
\end{aligned}
$$




$$
\begin{aligned}
& z_{1}=\left[3 e F_{9} F_{13}^{4}+3 f F_{9} F_{13}^{3} F_{15}+3 e\left(F_{15}-1\right) F_{9} F_{13}^{2} F_{15}+3 f\left(F_{15}^{3}-F_{15}^{2}-F_{15}\right.\right. \\
& \left.+1) F_{9} F_{13}\right] F_{11}+3 a F_{7} F_{9} F_{13}^{4}+\left[\left(3 f F_{9} F_{12}+3 c F_{8} F_{9}+a F_{9}^{2}+4 a\right) F_{14}+\left(3 c F_{7} F_{9}+F_{9}^{2} F_{10}\right.\right. \\
& \left.\left.-3 F_{9}-2 F_{10}\right) F_{15}\right] F_{13}^{3}+\left[\left(2\left(3 a F_{8} F_{9}+b F_{9}^{2}-2 b+2 c F_{9}^{2}+3 e F_{9} F_{12}-3 F_{9} \alpha+2 c\right) F_{15}\right.\right. \\
& \left.\left.+3 F_{9} \alpha-c+2 b-c F_{9}^{2}-3 e F_{9} F_{12}-3 a F_{8} F_{9}-b F_{9}^{2}\right) F_{14}+3 a F_{7} F_{9} F_{15}\left(F_{15}-1\right)\right] F_{13}^{2} \\
& +\left[\left(3\left(3 f F_{9} F_{12}+3 c F_{8} F_{9}+4 a+a F_{9}^{2}\right) F_{15}^{2}+2\left(-2 a F_{9}^{2}-3 c F_{8} F_{9}-5 a-3 f F_{9} F_{12}\right) F_{15}\right.\right. \\
& \left.\left.-3 f F_{9} F_{12}+3 a F_{9}^{2}-3 c F_{8} F_{9}\right) F_{14}+\left(3 c F_{7} F_{9}+F_{9}^{2} F_{10}-3 F_{9}-2 F_{10}\right)\left(F_{15}^{3}-F_{15}^{2}-F_{15}+1\right)\right] F_{13} \\
& +c\left(F_{9}^{2}+1\right)\left(8 F_{15}^{3}-9 F_{15}^{2}+1\right) F_{14} \\
& z_{2}=F_{14}\left[\left((b+2 c) F_{13}^{2}+a\left(-2+3 F_{15}\right) F_{13}+12 c F_{15}^{2}-9 c F_{15}\right) F_{14}+F_{10} F_{13}\left(-2 F_{15}-1+3 F_{15}^{2}\right.\right. \\
& \left.\left.+F_{13}^{2}\right)\right) F_{9}^{2}+\left(\left(3 F_{8} F_{13}\left(a F_{13}-c+3 c F_{15}\right)+3 F_{12} F_{13}\left(e F_{13}+3 f F_{15}-f\right)-3 \alpha F_{13}^{2}\right) F_{14}\right. \\
& +3 F_{13}\left(\left(c F_{7}+f F_{11}-1\right) F_{13}^{2}+\left(a F_{7}+e F_{11}\right)\left(2 F_{13} F_{15}-F_{13}\right)+\left(3 F_{15}+1\right)\left(F_{15}-1\right)\left(c F_{7}\right.\right. \\
& \left.\left.\left.+f F_{11}-1\right)\right)\right) F_{9}+\left(2(-b+c) F_{13}^{2}+a\left(-5+12 F_{15}\right) F_{13}+3 c\left(4 F_{15}-3\right) F_{15}\right) F_{14} \\
& \left.-2 F_{10} F_{13}\left(-2 F_{15}-1+3 F_{15}^{2}+F 13^{2}\right)\right] \\
& z_{3}=F_{14}^{2}\left[\left(\left(a F_{13}+8 c F_{15}-3 c\right) F_{14}+3 F_{10} F_{13} F_{15}-F_{10} F_{13}\right) F_{9}^{2}+\left(\left(3 f F_{12}+3 c F_{8}\right) F_{13} F_{14}\right.\right. \\
& \left.+3\left(\left(a F_{13}-c+3 c F_{15}\right) F_{7}+\left(e F_{13}+3 f F_{15}-f\right) F_{11}+1-3 F_{15}\right) F_{13}\right) F_{9}+\left(8 c F_{15}+4 a F_{13}\right. \\
& \left.-3 c) F_{14}-6 F_{10} F_{13} F_{15}+2 F_{10} F_{13}\right] \\
& z_{4}=F_{14}^{3}\left[\left(2 c F_{14}+F_{10} F_{13}\right) F_{9}^{2}+\left(3 f F_{11} F_{13}-3 F_{13}+3 c F_{7} F_{13}\right) F_{9}+2 c F_{14}-2 F_{10} F_{13}\right] .
\end{aligned}
$$

Para verificar que o polinômio $\xi_{2}$ não é identicamente nulo, vamos verificar o termo $z_{4}$. Substituindo $F_{14}$ e logo após $F_{7}$ e $F_{11}$ na expressão de $z_{4}$ temos que este se anula quando 
$F_{13}$ for igual a $s_{1}$ que é dado por

$$
s_{1}=-\frac{c e\left(a P_{1} Q_{2}+P_{2} Q_{1}\right)}{\left(\left(\left(F_{10} F_{9}^{2}-3 F_{9}-2 F_{10}\right)(a f-c e) Q_{2}+3 f F_{9} P_{2}\right) Q_{1}+3 c e F_{9} P_{1} Q_{2}\right) \sqrt{F_{9}^{2}+1}},
$$

onde, denotando $\widetilde{F}_{i}=\frac{F_{i}}{e}$ para $i=1,3,5$,

$$
\begin{aligned}
P_{1} & =\widetilde{F}_{1} W^{2}+\widetilde{F}_{3} W+\widetilde{F}_{5} \\
P_{2} & =a c \alpha\left(c \alpha\left(-W\left(a^{2}+b c\right)+8 W^{2} c \alpha-2 c \alpha\right)-12 W(c \alpha)^{2}\right) \\
Q_{1} & =-16(c \alpha)^{2}\left(f_{4} W^{2}+f_{5} W+f_{6}\right) \\
Q_{2} & =8(c \alpha)^{3} .
\end{aligned}
$$

O termo $s_{1}$ estará bem definido se supormos que $f \neq 0$ e $\frac{c e}{f} \neq s_{2}$ onde

$$
s_{2}=\frac{Q_{1}\left(a F_{10} F_{9}^{2} Q_{2}+3 F_{9} P_{2}-3 a F_{9} Q_{2} a-2 a F_{10} Q_{2}\right)}{Q_{2}\left(-3 F_{9} Q_{1}+F_{10} F_{9}^{2} Q_{1}-2 F_{10} Q_{1}-3 F_{9} P_{1}\right)}
$$

O termo $s_{2}$ depende de $a, b, c$ e $\alpha$. E a fração $c e / f$ pode ser positiva ou negativa, o mesmo acontecendo para $s_{2}$. Assim, supondo que $s_{2}$ é um número real, substituindo o valor de $F_{13}$ em $F_{13}=s_{1}$ obtemos que o único ponto onde essa igualdade é satisfeita é quando $\frac{c e}{f}=s_{3}$ onde

$$
\begin{aligned}
s_{3} & =\left[a Q_{1}\left(a F_{10} F_{9}^{2} Q_{2}+3 F_{9} P_{2}-3 a F_{9} Q_{2}-2 a F_{10} Q_{2}\right)\left(F_{9}+\left(F_{9}^{2}+1\right)^{1 / 2}\right)\right]\left[\left(\left(a Q _ { 2 } \left(-3 F_{9}\right.\right.\right.\right. \\
& \left.\left.\left.-2 F_{10}+F_{10} F_{9}^{2}\right)\left(F_{9}+\left(F_{9}^{2}+1\right)^{1 / 2}\right)\right)-2 P_{2}\right) Q_{1}+\left(-3 a F_{9}^{2} P_{1}-3 a\left(F_{9}^{2}+1\right)^{1 / 2} F_{9} P_{1}\right. \\
& \left.\left.-2 a P_{1}\right) Q_{2}\right]^{-1}
\end{aligned}
$$

e $s_{3}$ depende de $a, b, c$ e $\alpha$.

Das observações acima provamos o seguinte lema.

Lema 26. Seja $Z_{\lambda, R}$ a FCR do Lema 23. Se $f \neq 0, \frac{c e}{f} \neq s_{2}$, e distinto de $s_{3}$ onde $s_{2}$ é dado por (3.57) e $s_{3}$ dado por (3.58), e $\beta$ não é raiz do polinômio (3.56) então vale o item BT.2) do Teorema 22. 
Seja a função

$$
R(x, y, d, \lambda)=\left(Z_{1}(x, y, d, \lambda), Z_{2}(x, y, d, \lambda), \operatorname{det}(A), \operatorname{tr}(A)\right)=\left(R_{1}, R_{2}, R_{3}, R_{4}\right)
$$

Sua regularidade vem do fato de que o determinante da seguinte matriz é não-nulo

$$
\operatorname{Jac}(R)=\left(\begin{array}{cccc}
\frac{\partial R_{1}}{\partial x} & \frac{\partial R_{1}}{\partial y} & \frac{\partial R_{1}}{\partial d} & \frac{\partial R_{1}}{\partial \lambda} \\
\frac{\partial R_{2}}{\partial x} & \frac{\partial R_{2}}{\partial y} & \frac{\partial R_{2}}{\partial d} & \frac{\partial R_{2}}{\partial \lambda} \\
\frac{\partial R_{3}}{\partial x} & \frac{\partial R_{3}}{\partial y} & \frac{\partial R_{3}}{\partial d} & \frac{\partial R_{3}}{\partial \lambda} \\
\frac{\partial R_{4}}{\partial x} & \frac{\partial R_{4}}{\partial y} & \frac{\partial R_{4}}{\partial d} & \frac{\partial R_{4}}{\partial \lambda}
\end{array}\right)
$$

Calculando esse determinante e substituindo os valores de $x$ por $F_{7} \beta+F_{8}, y$ por $F_{9}, d$ por $F_{10} \beta-a$ e $\lambda$ por $F_{11} \beta+F_{12}$, e simplificando, obtemos

$$
\operatorname{det}(\operatorname{Jac}(R))=-\frac{\gamma_{1}\left(F_{9}+\sqrt{1+F_{9}^{2}}\right) \beta}{32\left(1+F_{9}^{2}\right)^{9 / 2}}-\frac{\gamma_{2}\left(F_{9}+\sqrt{1+F_{9}^{2}}\right)}{32\left(1+F_{9}^{2}\right)^{9 / 2}}
$$

portanto, teremos a regularização da funçao $R$ se

$$
\beta \neq-\frac{\gamma_{2}}{\gamma_{1}}
$$

onde

$$
\begin{aligned}
\gamma_{1} & =(c e-a f)\left[\left(2 a F_{9}^{3} F_{10}+6 c\left(a F_{7}+e F_{11}\right) F_{9}^{2}+3 a F_{9} F_{10}+a f F_{11}-a+a c F_{7}\right) \sqrt{1+F_{9}^{2}}+a F_{10}\right. \\
& \left.+2 a F_{9}^{4} F_{10}+6 c\left(a F_{7}+e F_{11}\right) F_{9}^{3}+4 a F_{9}^{2} F_{10}+\left(a f F_{11}-a+4 a c F_{7}+3 c e F_{11}\right) F_{9}\right] \\
\gamma_{2} & =(a f-c e)\left[\left(2\left(-b c+2 a^{2}\right) F_{9}^{3}+6 c\left(-e F_{12}+\alpha-a F_{8}\right) F_{9}^{2}+\left(5 a^{2}+4 b c\right) F_{9}-a c F_{8}\right.\right. \\
& \left.-a f F_{12}\right) \sqrt{1+F_{9}^{2}}+2\left(-b c+2 a^{2}\right) F_{9}^{4}+3 c F_{9}\left(-e F_{12}+\alpha-a F_{8}\right)\left(2 F_{9}^{2}+1\right)+\left(3 b c+7 a^{2}\right) F_{9}^{2} \\
& \left.-a F_{9}\left(f F_{12}+c F_{8}\right)+2 a^{2}+2 b c\right] .
\end{aligned}
$$

Assim, provamos o seguinte lema. 
Lema 27. Seja $Z_{\lambda, R}$ a FCR do Lema 23, se $\beta \in \mathbb{R}^{+}$não é solução de (3.60), então vale o item BT.3) do Teorema 22.

Assim, resumimos os cálculos e resultados acima obtidos no próximo teorema que caracteriza a existência de um ponto de Bogdanov-Takens na regularização de uma família descontínua que seja do tipo $\mathcal{F}_{4}$.

Teorema 28. Seja $Z_{\lambda, R}$ a FCD do Lema 23. Se $\beta \in \mathbb{R}^{+}$não é raiz dos polinômios (3.46) $e(3.56), e \neq 0, f \neq 0, \frac{f}{e} \neq f_{s}$, onde $f_{s}$ é dado por $(3.54), \widetilde{W}_{+}<0$ quando $a^{2}+b c>0$, $\widetilde{W}_{-}>0$ quando $a^{2}+b c<0$, onde $\widetilde{W}_{ \pm}$são dados por $(3.51), \frac{c e}{f}$ diferente de $s_{2}$ e $s_{3}$, onde $s_{2}$ é dado por (3.57) e $s_{3}$ dado por (3.58), então $Z_{\lambda, R}$ apresenta uma bifurcação de BogdanovTakens no ponto $p_{B T}$, dado por (3.41).

Demonstração. A prova é uma aplicação imediata dos lemas anteriores.

\subsection{Diagramas de bifurcação de Filippov-Bogdanov-Takens}

Nesta seção vamos apresentar os diagramas referentes à bifurcação de Filippov-BogdanovTakens. As contas para a prova da existência de um ponto de Bogdanov-Takens na família regularizada estão apresentadas na seção anterior.

Como observado anteriormente, as curvas de bifurcação para uma família de campos vetoriais descontínuos são os limites das curvas de bifurcação da família regularizada quando $\varepsilon$ vai a zero, onde $\varepsilon$ é o parâmetro da regularização. Uma vez que sabemos desse fato, verificamos que o diagrama de bifurcação de Filippov-Bogdanov-Takens deve ser equivalente ao conhecido diagrama de bifurcação de Bogdanov-Takens para o caso de famílias regulares de campos vetoriais.

A próxima figura apresenta o diagrama de bifurcação de Bogdanov-Takens para a seguinte família a 2-parâmetros de campos vetoriais regulares

$$
\begin{aligned}
& x^{\prime}=y \\
& y^{\prime}=\beta_{2}+\beta_{1} x+x^{2}+x y .
\end{aligned}
$$



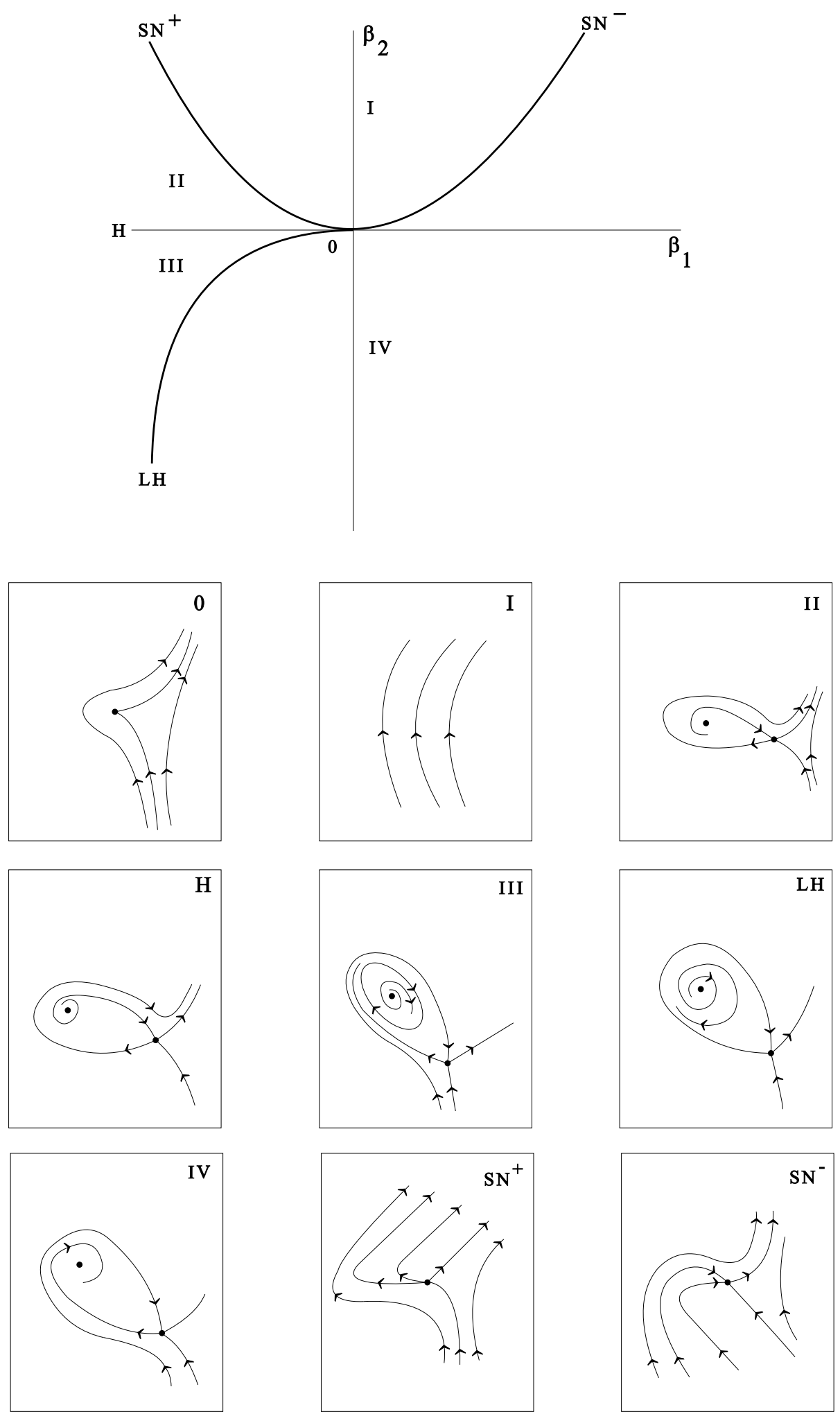

figura 24: Diagrama de bifurcação de Bogdanov-Takens 
Uma vez que temos o diagrama de bifurcação de Bogdanov-Takens para uma família regular de campos vetoriais, temos que tal diagrama de bifurcação também é válido para a família de campos vetoriais regularizados do capítulo anterior. Assim, fixado um $\varepsilon>0$ pequeno o diagrama de bifurcação de uma família regularizada, associada a uma família descontínua que seja do tipo $\mathcal{F}_{4}$, é exatamente o diagrama de bifurcação dado acima. Quando $\varepsilon>0$ vai a zero o seu limite será o diagrama da família descontínua. Além disso, quando $\varepsilon$ vai a zero o limite das curvas $H$ e $L H$ são curvas no plano dos parâmetros.

No capítulo anterior verificamos que a regularização da seguinte família descontínua de campos vetoriais $Z_{\lambda}=\left(X_{\lambda}, Y_{\lambda}\right)$ onde

$$
\begin{aligned}
& X_{\lambda}(x, y)=(a x+b y+e \lambda, c x+d y+f \lambda), \\
& Y_{\lambda}(x, y)=(\alpha, \beta)
\end{aligned}
$$

que seja do tipo $\mathcal{F}_{4}$, com hipóteses sobre os seus coeficientes, possui um único ponto de Bogdanov-Takens, supondo que $d$ e $\lambda$ são os parâmetros considerados. Assim, temos que se $Z_{\lambda}$ é do tipo $\mathcal{F}_{4}$ então a bifurcação que ocorre nesse caso é uma bifurcação do tipo BogdanovTakens para o caso descontínuo.

O próximo diagrama contém a bifurcação de Filippov-Bogdanov-Takens. No diagrama aparece o desdobramento de três tipos de bifurcação, a saber, os tipos $\mathcal{F}_{1}, \mathcal{F}_{2}$ e $\mathcal{F}_{4}$. Na curva $L H$ ocorre a conexão de sela, ou seja, o caso $\mathcal{F}_{4}$, em $I I I, H$ e $I I$ estão os desdobramentos de $\mathcal{F}_{1}$, em $I V$ temos o $\mathcal{F}_{2}$, e em $I$ temos $\mathcal{F}_{1}$ ou $\mathcal{F}_{2}$, após a bifurcação de sela-nó. O fato de $\mathcal{F}_{1}$ estar em vários lugares se dá, por exemplo, ao fato que não é possível determinar visualmente quando ocorre a bifurcação de Hopf na família descontínua, ou seja, não conseguimos verificar variando o parâmetro $\lambda$ o nascimento de um ciclo limite em $Z_{\lambda}$, tal fenômeno só é possível de ser visualizado na regularização. 

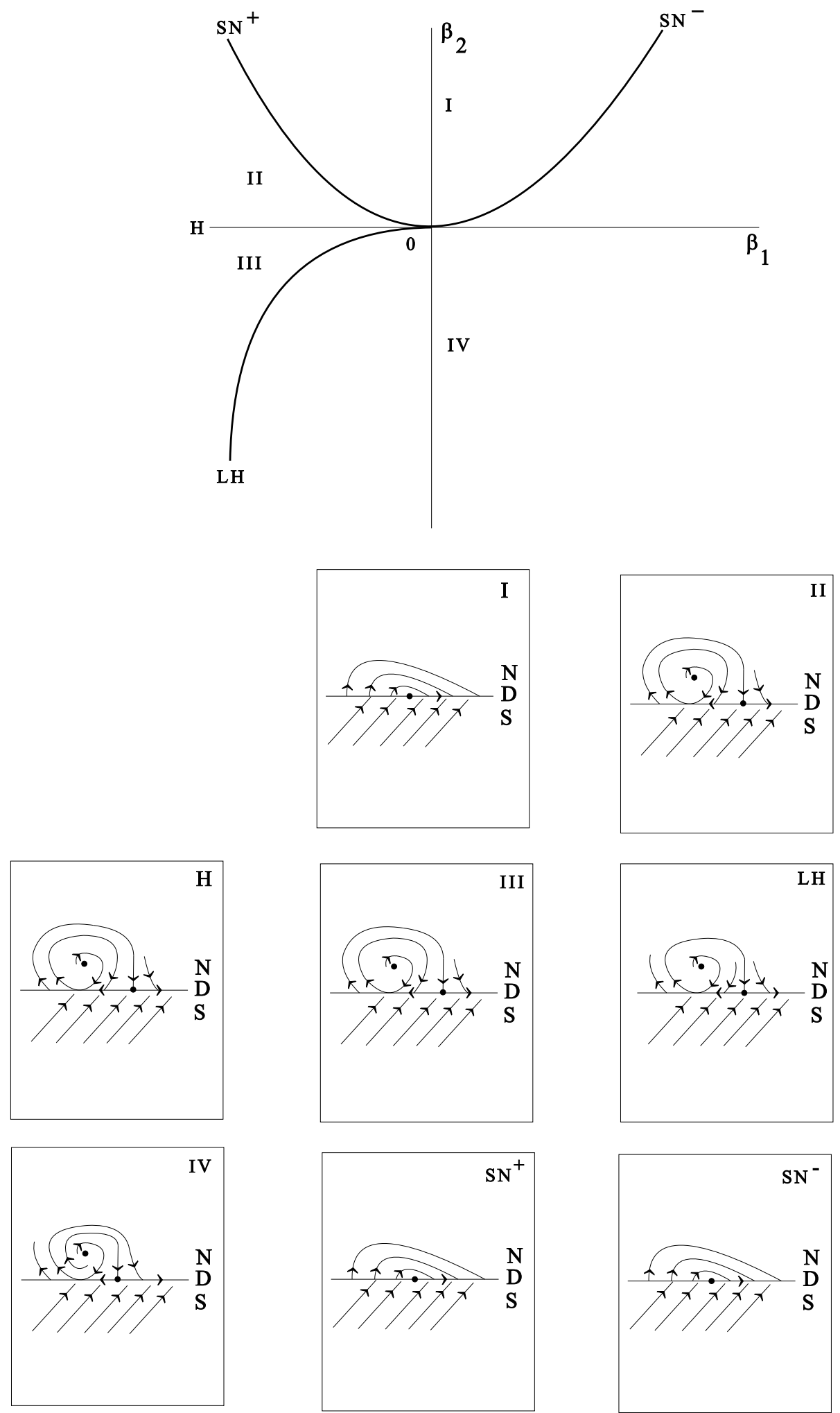

figura 25: Diagrama de bifurcação de Filippov-Bogdanov-Takens 
Podemos simplificar o que ocorre no diagrama da bifurcação de Filippov-BogdanovTakens. Para isso, vamos considerar o diagrama da figura 24 e um círculo $C$ de raio $R>0$ centrado na origem do plano $(d, \lambda)$. A próxima figura ilustra esse círculo.

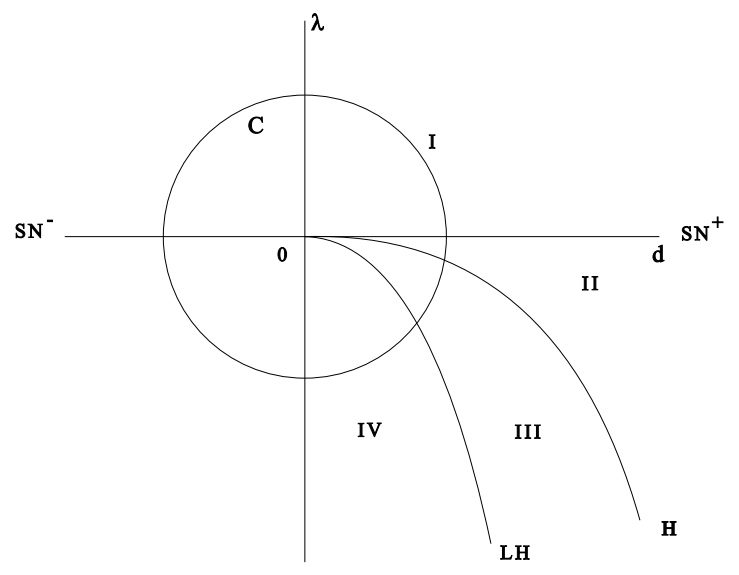

figura 26: Diagrama de Filippov-Bogdanov-Takens com o círculo $C$

Assim, consideramos que o círculo é parametrizado por

$$
C(\nu, R)=(R \cos (\nu), R \operatorname{sen}(\nu))
$$

$\operatorname{com} \nu \in[0,2 \pi]$ e onde o sentido horário será convencionado como sendo o positivo. Portanto, partindo de $(R, 0)$ temos um ponto de sela-nó que se desdobra em uma sela e um nó, logo após o nó vira um foco e esse foco torna um ponto de Hopf seguido pela criação de um ciclo limite. Seguindo adiante temos uma conexão de sela e logo após o desaparecimento dessa conexão não se vê mais o ciclo limite e o foco existente vira nó para então colidir com a sela e na região $I$ não há singularidades.

A próxima figura sintetiza com cores o que escrevemos acima, notamos que a figura se repete indefinidamente, uma vez que podemos estender o domínio de $\nu$ para os reais. 

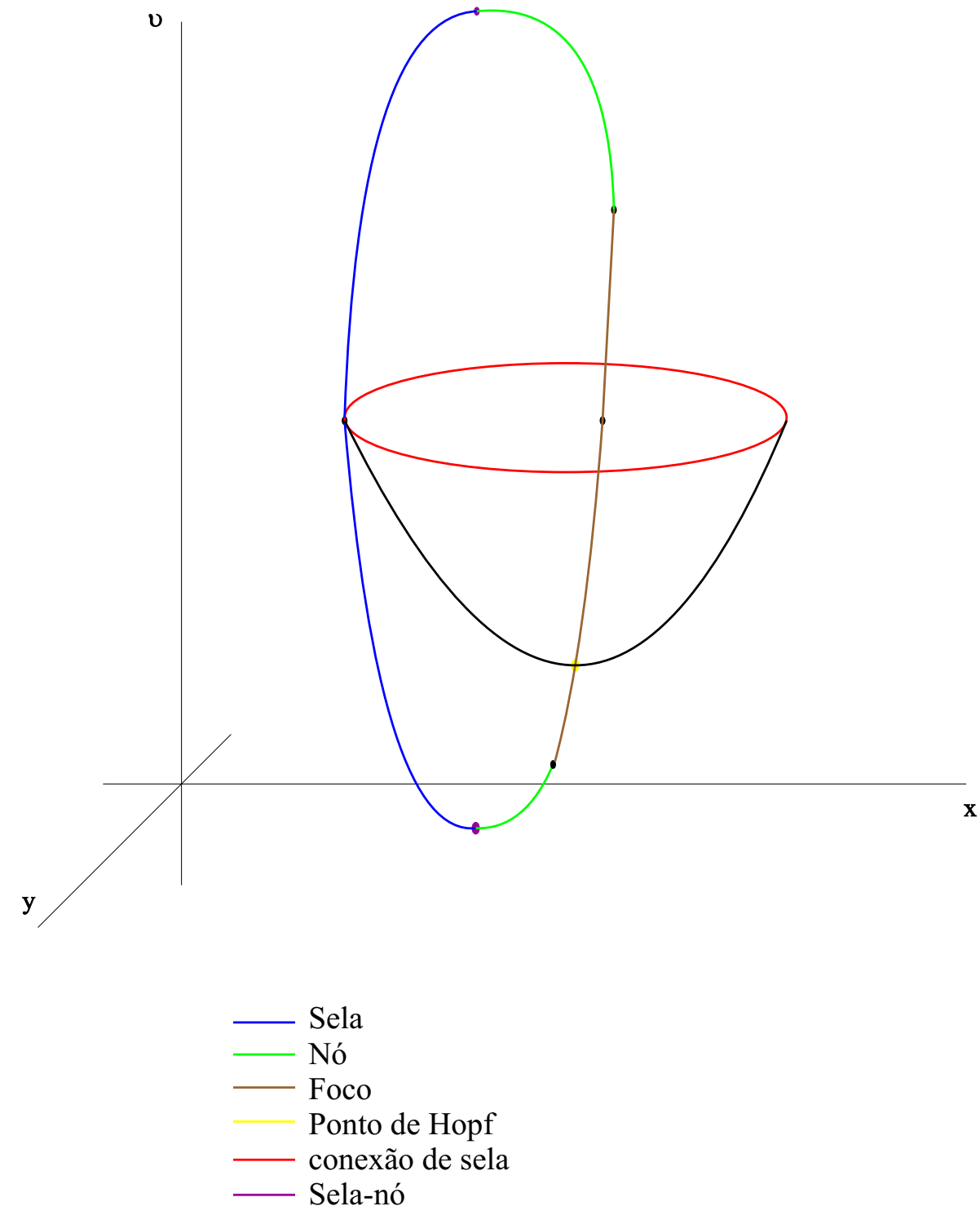

figura 27: Diagrama de bifurcação

\subsection{Exemplo numérico}

Nesta seção analisaremos um caso particular de uma família de campos vetoriais descontínuos que pertence ao caso $\mathcal{F}_{4}$.

Seja $Z_{\lambda}(x, y)=\left(X_{\lambda}(x, y), Y_{\lambda}(x, y)\right)$ a família de campos vetoriais descontínuos a um parâmetro dada por

$$
\begin{aligned}
X_{\lambda}(x, y) & =(a x+b y+\lambda,-b x+a y+\lambda) \\
Y_{\lambda}(x, y) & =(\alpha, \beta)
\end{aligned}
$$


onde estamos supondo que $a>0, b>0, \alpha>0$ e $\beta>0$. Vamos supor que o conjunto de descontinuidade é o eixo $x$.

Nosso objetivo não é provar os lemas da seção anterior para este exemplo, por se tratar de um subcaso da família tratada anteriormente valem os resultados daquela seção. Ao invés disto, apenas vamos fazer as contas necessárias para encontrar o ponto de Bogdanov-Takens. Faremos isto para verificar que mesmo com essa simplificação da família descontínua, as contas ficam grandes, e se provássemos os lemas da seção anterior, não teríamos contas menores que aquelas, isto pelo tamanho da expressão do ponto de Bogdanov-Takens.

Como anteriormente queremos que $X_{\lambda}$ tenha um foco repulsor em $N$ para valores negativos e pequenos do parâmetro $\lambda$.

Notamos que as condições iniciais para que a família seja do tipo foco no bordo estão satisfeitas. De fato, o traço e o determinante da parte linear do campo $X_{\lambda}$ são dados respectivamente por $2 a>0$ e $a^{2}+b^{2}>0$. Como $b>0$ então as órbitas do foco teem rotação horária. Além disso, o discriminante do polinômio característico da parte linear do campo $X_{\lambda}$ vale $-4 b^{2}<0$.

As singularidades de $X_{\lambda}$ satisfazem

$$
\begin{aligned}
& x=\frac{(b-a) \lambda}{a^{2}+b^{2}} \\
& y=\frac{-(a+b) \lambda}{a^{2}+b^{2}} .
\end{aligned}
$$

Como $-(a+b)<0$ temos que para valores negativos e pequenos do parâmetro o foco aparece na região $N$, como esperávamos.

O ponto $p_{0}=(\lambda / b, 0)$ é onde ocorre a tangência entre uma órbita que sai do foco de $X_{\lambda}$ com o conjunto de descontinuidade para $\lambda<0$ pequeno.

O campo de Filippov, $F_{Z_{\lambda}}$ é dado por

$$
F_{Z_{\lambda}}(x, y)=\left(\frac{(a \beta+b \alpha) x+(\beta-\alpha) \lambda}{\beta+b x-a y-\lambda}, 0\right),
$$

cujas singularidades são pontos no eixo- $x$ que satisfazem

$$
x=\frac{(\alpha-\beta) \lambda}{a \beta+b \alpha} .
$$

Pelo lema 11 obtemos as caracterizações de três dos quatro casos a analisar: 
a) se $G(a, b)=0$ onde $G$ é a função dada por

$$
G(a, b)=\frac{a \pi}{2 b}+\ln \left(\frac{a}{b}\right)
$$

então a família $Z_{\lambda}$ é do tipo $\mathcal{F}_{4}$.

b) se $G(a, b)<0$ então a família $Z_{\lambda}$ é do tipo $\mathcal{F}_{1}$

c) se $G(a, b)>0$ então a família $Z_{\lambda}$ é do tipo $\mathcal{F}_{2}$.

Notamos que não estamos considerando o sinal de $a \beta+b \alpha$ para distinguir os quatro casos de foco no bordo. Isto se deve ao fato de $a, b, \alpha$ e $\beta$ serem todos positivos, implicando que $a \beta+b \alpha$ é sempre positivo.

A caracterização do caso $\mathcal{F}_{3}$ se dá pelo fato da isóclina que anula o primeiro termo da família $X_{\lambda}$, ter coeficiente angular estritamente positivo. Da expressão do campo temos que essa isóclina é a reta dada por $-(\lambda+a x) / b$. Portanto, o caso $\mathcal{F}_{3}$ ocorre quando $-a / b>0$, o que não acontece uma vez que $a$ e $b$ são positivos.

Assim, o campo $Z_{\lambda}$ dado por (3.62), nas hipóteses iniciais para $a, b, \alpha$ e $\beta$, não apresenta o caso $\mathcal{F}_{3}$.

Considerando o plano $(a, b)$ a próxima figura apresenta as regiões onde a função $G(a, b)$ se anula, é positiva ou negativa, o que, consequentemente, indica onde ocorrem os casos $\mathcal{F}_{1}$, $\mathcal{F}_{2}$ e $\mathcal{F}_{4}$. A função $G(a, b)=0$ tem como raiz a reta dada por $b=2,107299 a$. Para valores de $a$ e $b$ que estejam na região compreendida entre o eixo horizontal, $a$, e a reta que anula $G$ temos que $G(a, b)>0$, e será negativa na região complementar, considerando o primeiro quadrante do plano $(a, b)$.

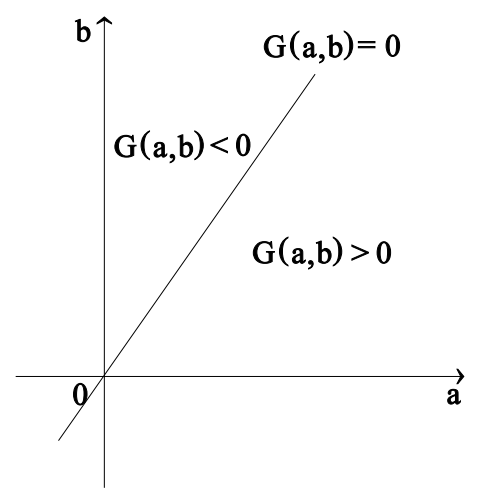

figura 28: Regiões onde $G$ se anula, é positiva ou negativa. 
Agora que caracterizamos os casos, vamos nos ater ao caso mais interessante que é o caso $\mathcal{F}_{4}$. Portanto, vamos supor que $G(a, b)=0$.

O segundo parâmetro que iremos considerar é o coeficiente $b$, pois a derivada de $G(a, b)$ com relação a $b$ vale $-\frac{a \pi}{2 b^{2}}-\frac{1}{b}$ que nunca se anula, uma vez que $a$ e $b$ são positivos.

A função de transição a ser usada é a mesma das seções anteriores, ou seja, para $\varepsilon>0$

$$
\varphi(y)=\frac{1}{2}+\frac{y}{2 \sqrt{y^{2}+\varepsilon^{2}}} .
$$

A família regularizada, $Z_{\lambda, \varepsilon}$, tem a seguinte expressão

$$
\begin{aligned}
Z_{\lambda, \varepsilon}(x, y)= & \left(\left(\frac{1}{2}-\frac{y}{2 \sqrt{y^{2}+\varepsilon^{2}}}\right) \alpha+\left(\frac{1}{2}+\frac{y}{2 \sqrt{y^{2}+\varepsilon^{2}}}\right)(a x+b y+\lambda),\right. \\
& \left.\left(\frac{1}{2}-\frac{y}{2 \sqrt{y^{2}+\varepsilon^{2}}}\right) \beta+\left(\frac{1}{2}+\frac{y}{2 \sqrt{y^{2}+\varepsilon^{2}}}\right)(-b x+a y+\lambda),\right) .
\end{aligned}
$$

Efetuando a seguinte mudança de variáveis e reescalonamento dos parâmetros, usando ainda os mesmos símbolos das variáveis e parâmetros originais, $x=\varepsilon x, y=\varepsilon y, \alpha=\varepsilon \alpha, \beta=\varepsilon \beta$, e $\lambda=\varepsilon \lambda$, obtemos

$$
\begin{aligned}
Z_{\lambda, \varepsilon}(x, y)= & \left(\left(\frac{1}{2}-\frac{y}{2 \sqrt{y^{2}+1}}\right) \alpha+\left(\frac{1}{2}+\frac{y}{2 \sqrt{y^{2}+1}}\right)(a x+b y+\lambda),\right. \\
& \left.\left(\frac{1}{2}-\frac{y}{2 \sqrt{y^{2}+1}}\right) \beta+\left(\frac{1}{2}+\frac{y}{2 \sqrt{y^{2}+1}}\right)(-b x+a y+\lambda)\right) .
\end{aligned}
$$

O traço e o determinante Jacobiano de $Z_{\lambda, \varepsilon}$ são dados, respectivamente por

$$
\begin{aligned}
\operatorname{tr} & =\frac{2 a\left(y^{2}+1\right)^{3 / 2}+2 a y^{3}+3 a y-\beta-b x+\lambda}{2\left(y^{2}+1\right)^{3 / 2}} \\
\operatorname{det} & =\frac{\left(\sqrt{y^{2}+1}+y\right)\left(-a \beta-b \alpha+\left(a^{2}+b^{2}\right)\left(y^{3}+\left(y^{2}+1\right)^{3 / 2}+2 y\right)+(a+b) \lambda\right)}{4\left(y^{2}+1\right)^{2}} .
\end{aligned}
$$

Calculando as soluções em $x, y, b$ e $\lambda$ do sistema $\left(Z_{\lambda, \varepsilon}(x, y)\right.$, tr, det $)=(0,0,0,0)$ obtemos 6 raízes onde 4 dessas raízes são complexas, e não nos interessa, e 2 são reais. As expressões 
de $b$ dessas duas raízes reais são dadas por

$$
b=\frac{\alpha \pm \sqrt{\alpha^{2}+\beta^{2}}}{\beta} .
$$

Como $\sqrt{\alpha^{2}+\beta^{2}}>\alpha$ e do fato que $b$ deve ser positivo, segue que o ponto de Bogdanov-Takens é tal que $b=\left(\alpha+\sqrt{\alpha^{2}+\beta^{2}}\right) \beta^{-1}$.

Assim, o ponto de Bogdanov-Takens é dado por

$$
\begin{aligned}
x_{B T} & =\frac{g_{1} S^{2}+g_{2} S+g_{3}}{4 \beta\left(\beta^{2}+(2 \alpha+\beta)\left(\alpha+\sqrt{\alpha^{2}+\beta^{2}}\right)\right)\left(g_{4} S^{2}+g_{5} S+g_{6}\right)} \\
y_{B T} & =-\frac{a^{2} S^{2}-\beta^{2}}{4 a \beta S^{2}} \\
\lambda_{B T} & =-\frac{h_{1}\left(\alpha+\sqrt{\alpha^{2}+\beta^{2}}\right)+h_{2}}{32 h_{3}} \\
b_{B T} & =\frac{\alpha+\sqrt{\alpha^{2}+\beta^{2}}}{\beta}
\end{aligned}
$$

onde

$$
\begin{gathered}
S=\frac{\left(\left(36 a^{2} \beta^{2}+216 \beta^{4}+a^{4}+24 \beta^{3} \sqrt{3 a^{2}+81 \beta^{2}}\right) a^{2}\right)^{1 / 3}}{12 a \beta}+\frac{a}{12 \beta} \\
+\frac{a\left(24 \beta^{2}+a^{2}\right)}{12 \beta\left(\left(36 a^{2} \beta^{2}+216 \beta^{4}+a^{4}+24 \beta^{3} \sqrt{3 a^{2}+81 \beta^{2}}\right) a^{2}\right)^{1 / 3}} \\
g_{1}=-128 a \beta^{8}+384 a\left(\alpha+\sqrt{\alpha^{2}+\beta^{2}}\right) \beta^{7}+\left(-512 a \alpha\left(\alpha+\sqrt{\alpha^{2}+\beta^{2}}\right)+80 a^{3}\right) \beta^{6} \\
+160 a^{3}\left(\alpha+\sqrt{\alpha^{2}+\beta^{2}}\right) \beta^{5}+\left(24 a^{5}-80 a^{3} \alpha\left(\alpha+\sqrt{\alpha^{2}+\beta^{2}}\right)\right) \beta^{4}+8 a^{5}\left(\alpha+\sqrt{\alpha^{2}+\beta^{2}}\right) \beta^{3} \\
+\left(16 a^{5} \alpha\left(\alpha+\sqrt{\alpha^{2}+\beta^{2}}\right)+a^{7}\right) \beta^{2}+a^{7} \alpha\left(\alpha+\sqrt{\alpha^{2}+\beta^{2}}\right) \\
g_{2}=-64 \beta^{9}+128\left(\alpha+\sqrt{\alpha^{2}+\beta^{2}}\right) \beta^{8}+\left(32 a^{2}-192 \alpha\left(\alpha+\sqrt{\alpha^{2}+\beta^{2}}\right)\right) \beta^{7} \\
+224 a^{2}\left(\alpha+\sqrt{\alpha^{2}+\beta^{2}}\right) \beta^{6}+\left(-192 a^{2} \alpha\left(\alpha+\sqrt{\alpha^{2}+\beta^{2}}\right)+36 a^{4}\right) \beta^{5} \\
+16 a^{4}\left(\alpha+\sqrt{\alpha^{2}+\beta^{2}}\right) \beta^{4}+\left(20 a^{4} \alpha\left(\alpha+\sqrt{\alpha^{2}+\beta^{2}}\right)+2 a^{6}\right) \beta^{3}+2 a^{6} \alpha\left(\alpha+\sqrt{\alpha^{2}+\beta^{2}}\right) \beta
\end{gathered}
$$




$$
\begin{aligned}
g_{3} & =96 a\left(\alpha+\sqrt{\alpha^{2}+\beta^{2}}\right) \beta^{7}+\left(16 a^{3}-96 a \alpha\left(\alpha+\sqrt{\alpha^{2}+\beta^{2}}\right)\right) \beta^{6}+8 a^{3}\left(\alpha+\sqrt{\alpha^{2}+\beta^{2}}\right) \beta^{5} \\
& +\left(8 a^{3} \alpha\left(\alpha+\sqrt{\alpha^{2}+\beta^{2}}\right)+a^{5}\right) \beta^{4}+a^{5} \alpha\left(\alpha+\sqrt{\alpha^{2}+\beta^{2}}\right) \beta^{2} \\
g_{4} & =96 a^{2} \beta^{4}+24 a^{4} \beta^{2}+a^{6} \\
g_{5} & =64 a \beta^{5}+36 a^{3} \beta^{3}+2 a^{5} \beta \\
g_{6} & =16 \beta^{6}+16 a^{2} \beta^{4}+a^{4} \beta^{2}
\end{aligned}
$$




$$
\begin{aligned}
& h_{1}=-96 a^{14} \beta^{5}-1720320 \beta^{15} a^{4}-131072 \beta^{17} a^{2}-492544 a^{8} \beta^{11}-\beta^{3} a^{16}-3488 a^{12} \beta^{7} \\
& -1712128 \beta^{13} a^{6}-65536 \beta^{19}-59904 a^{10} \beta^{9}-131072 \beta^{18} \alpha-4100096 \beta^{12} a^{6} \alpha \\
& -931840 \beta^{10} a^{8} \alpha-3932160 \beta^{16} a^{2} \alpha-104448 a^{10} \beta^{8} \alpha-7569408 \beta^{14} a^{4} \alpha-176 \beta^{4} a^{14} \alpha \\
& -6080 \beta^{6} a^{12} \alpha-16 \beta^{3} a^{14} \alpha^{2}-2 a^{16} \beta^{2} \alpha-15360 \beta^{7} a^{10} \alpha^{2}+4128768 \beta^{13} a^{4} \alpha^{2}-53248 \beta^{9} a^{8} \alpha^{2} \\
& +675840 \beta^{11} a^{6} \alpha^{2}-896 \beta^{5} a^{12} \alpha^{2}+3670016 \beta^{15} a^{2} \alpha^{2}+128 \beta^{7} a^{11} T^{3 / 2} \alpha+4096 a T^{3 / 2} \beta^{18} \\
& +8192 \beta^{17} a T^{3 / 2} \alpha+1456 a^{9} T^{3 / 2} \beta^{10}+107520 \beta^{13} a^{5} T^{3 / 2} \alpha+a^{13} T^{3 / 2} \beta^{6}+64 a^{11} T^{3 / 2} \beta^{8} \\
& +53760 a^{5} T^{3 / 2} \beta^{14}+14080 a^{7} T^{3 / 2} \beta^{12}+28160 \beta^{11} a^{7} T^{3 / 2} \alpha+114688 \beta^{15} a^{3} T^{3 / 2} \alpha \\
& +2 \beta^{5} a^{13} T^{3 / 2} \alpha+2912 \beta^{9} a^{9} T^{3 / 2} \alpha+57344 a^{3} T^{3 / 2} \beta^{16}+\left(-33792 \beta^{6} a^{11} \alpha^{2}-131136 a^{11} \beta^{8}\right. \\
& -5652480 \beta^{14} a^{5}-4449280 a^{7} \beta^{12}-262144 \beta^{18} a-146432 \beta^{8} a^{9} \alpha^{2}-7328 a^{13} \beta^{6} \\
& +264 \beta^{6} a^{12} T^{3 / 2} \alpha+276480 \beta^{12} a^{6} T^{3 / 2} \alpha+64768 \beta^{10} a^{8} T^{3 / 2} \alpha+4 \beta^{4} a^{14} T^{3 / 2} \alpha-196 a^{15} \beta^{4} \\
& +65536 \beta^{16} a^{2} T^{3 / 2} \alpha+372736 \beta^{14} a^{4} T^{3 / 2} \alpha+6272 \beta^{8} a^{10} T^{3 / 2} \alpha-1310720 \beta^{17} a \alpha-2 \beta^{2} a^{17} \\
& +10321920 \beta^{12} a^{5} \alpha^{2}-14745600 \beta^{15} a^{3} \alpha-950272 \beta^{16} a^{3}-228480 a^{11} \beta^{7} \alpha-32 \beta^{2} a^{15} \alpha^{2} \\
& -21626880 \beta^{13} a^{5} \alpha-2150400 a^{9} \beta^{9} \alpha-12800 \beta^{5} a^{13} \alpha+786432 \beta^{16} a \alpha^{2}+12845056 \beta^{14} a^{3} \alpha^{2} \\
& +3136 a^{10} T^{3 / 2} \beta^{9}+186368 a^{4} T^{3 / 2} \beta^{15}+32768 a^{2} T^{3 / 2} \beta^{17}+138240 a^{6} T^{3 / 2} \beta^{13}+2 a^{14} T^{3 / 2} \beta^{5} \\
& +32384 a^{8} T^{3 / 2} \beta^{11}+132 a^{12} T^{3 / 2} \beta^{7}-1856 \beta^{4} a^{13} \alpha^{2}-4 a^{17} \alpha \beta+1351680 \beta^{10} a^{7} \alpha^{2} \\
& \left.-360 \beta^{3} a^{15} \alpha-1148416 a^{9} \beta^{10}-10250240 a^{7} \beta^{11} \alpha\right) S+\left(-4208 a^{14} \beta^{5}-3342336 \beta^{15} a^{4}\right. \\
& -327680 \beta^{17} a^{2}-4179968 a^{8} \beta^{11}-104 \beta^{3} a^{16}-83840 a^{12} \beta^{7}-7974912 \beta^{13} a^{6}-855040 a^{10} \beta^{9} \\
& -24600576 \beta^{12} a^{6} \alpha-8945664 \beta^{10} a^{8} \alpha-5898240 \beta^{16} a^{2} \alpha-1566720 a^{10} \beta^{8} \alpha-25952256 \beta^{14} a^{4} \alpha \\
& -7392 \beta^{4} a^{14} \alpha-145920 \beta^{6} a^{12} \alpha-1024 \beta^{3} a^{14} \alpha^{2}-192 a^{16} \beta^{2} \alpha-143360 \beta^{7} a^{10} \alpha^{2} \\
& +19267584 \beta^{13} a^{4} \alpha^{2}+585728 \beta^{9} a^{8} \alpha^{2}+8650752 \beta^{11} a^{6} \alpha^{2}-21760 \beta^{5} a^{12} \alpha^{2}+5242880 \beta^{15} a^{2} \alpha^{2} \\
& +3840 \beta^{7} a^{11} T^{3 / 2} \alpha+2 \beta^{3} a^{15} T^{3 / 2} \alpha+23296 a^{9} T^{3 / 2} \beta^{10}-2 a^{18} \alpha+516096 \beta^{13} a^{5} T^{3 / 2} \alpha \\
& +72 a^{13} T^{3 / 2} \beta^{6}-\beta a^{18}+1920 a^{11} T^{3 / 2} \beta^{8}+258048 a^{5} T^{3 / 2} \beta^{14}+126720 a^{7} T^{3 / 2} \beta^{12} \\
& +253440 \beta^{11} a^{7} T^{3 / 2} \alpha+229376 \beta^{15} a^{3} T^{3 / 2} \alpha+144 \beta^{5} a^{13} T^{3 / 2} \alpha+a^{15} T^{3 / 2} \beta^{4} \\
& \left.-16 a^{16} \alpha^{2} \beta+46592 \beta^{9} a^{9} T^{3 / 2} \alpha+114688 a^{3} T^{3 / 2} \beta^{16}\right) S^{2}
\end{aligned}
$$




$$
\begin{aligned}
& h_{2}=-\beta^{4} a^{16}-65536 \beta^{20}-3040 a^{12} \beta^{8}-2050048 \beta^{14} a^{6}-3784704 \beta^{16} a^{4}-465920 a^{8} \beta^{12} \\
& -52224 a^{10} \beta^{10}-1966080 \beta^{18} a^{2}-88 a^{14} \beta^{6}-8 \beta^{5} a^{14} \alpha+2064384 \beta^{15} a^{4} \alpha \\
& -7680 a^{10} \beta^{9} \alpha+337920 \beta^{13} a^{6} \alpha-26624 \beta^{11} a^{8} \alpha+1835008 \beta^{17} a^{2} \alpha-448 \beta^{7} a^{12} \alpha \\
& +53760 a^{5} T^{3 / 2} \beta^{15}+14080 a^{7} T^{3 / 2} \beta^{13}+57344 a^{3} T^{3 / 2} \beta^{17}+4096 a T^{3 / 2} \beta^{19}+a^{13} T^{3 / 2} \beta^{7} \\
& +64 a^{11} T^{3 / 2} \beta^{9}+1456 a^{9} T^{3 / 2} \beta^{11}+\left(-\beta^{2} a^{18}-72960 a^{12} \beta^{8}-8 \beta^{3} a^{16} \alpha\right. \\
& -783360 a^{10} \beta^{10}-96 \beta^{4} a^{16}-4472832 a^{8} \beta^{12}+1920 a^{11} T^{3 / 2} \beta^{9}-12976128 \beta^{16} a^{4} \\
& +9633792 \beta^{15} a^{4} \alpha+2621440 \beta^{17} a^{2} \alpha-2949120 \beta^{18} a^{2}+126720 a^{7} T^{3 / 2} \beta^{13} \\
& -12300288 \beta^{14} a^{6}-3696 a^{14} \beta^{6}+4325376 \beta^{13} a^{6} \alpha-71680 a^{10} \beta^{9} \alpha+23296 a^{9} T^{3 / 2} \beta^{11} \\
& -512 \beta^{5} a^{14} \alpha+292864 \beta^{11} a^{8} \alpha+a^{15} T^{3 / 2} \beta^{5}+114688 a^{3} T^{3 / 2} \beta^{17}+258048 a^{5} T^{3 / 2} \beta^{15} \\
& \left.-10880 \beta^{7} a^{12} \alpha+72 a^{13} T^{3 / 2} \beta^{7}\right) S^{2}+\left(-16 \beta^{4} a^{15} \alpha-2 \beta^{3} a^{17}+5160960 \beta^{14} a^{5} \alpha\right. \\
& +675840 a^{7} \beta^{12} \alpha+138240 a^{6} T^{3 / 2} \beta^{14}+32384 a^{8} T^{3 / 2} \beta^{12}+132 a^{12} T^{3 / 2} \beta^{8} \\
& -16896 a^{11} \beta^{8} \alpha-5125120 a^{7} \beta^{13}-7372800 \beta^{17} a^{3}+186368 a^{4} T^{3 / 2} \beta^{16}-180 a^{15} \beta^{5} \\
& -928 \beta^{6} a^{13} \alpha-114240 a^{11} \beta^{9}+393216 \beta^{18} a \alpha-1075200 a^{9} \beta^{11}-655360 \beta^{19} a+2 a^{14} T^{3 / 2} \beta^{6} \\
& +32768 a^{2} T^{3 / 2} \beta^{18}-6400 a^{13} \beta^{7}+6422528 \beta^{16} a^{3} \alpha-10813440 \beta^{15} a^{5}+3136 a^{10} T^{3 / 2} \beta^{10} \\
& \left.-73216 a^{9} \beta^{10} \alpha\right) S \\
& h_{3}=\beta^{3}\left(\alpha \beta+\sqrt{\alpha^{2}+\beta^{2}} \beta+2\left(\alpha+\sqrt{\alpha^{2}+\beta^{2}}\right) \alpha+\beta^{2}\right)\left[\left(a^{14}+126720 \beta^{8} a^{6}+23296 \beta^{6} a^{8}\right.\right. \\
& \left.+258048 \beta^{10} a^{4}+114688 \beta^{12} a^{2}+1920 \beta^{4} a^{10}+72 \beta^{2} a^{12}\right) S^{2}+\left(32384 a^{7} \beta^{7}\right. \\
& \left.+32768 a \beta^{13}+132 a^{11} \beta^{3}+186368 a^{3} \beta^{11}+2 a^{13} \beta+138240 a^{5} \beta^{9}+3136 a^{9} \beta^{5}\right) S \\
& \left.+64 \beta^{4} a^{10}+14080 \beta^{8} a^{6}+53760 \beta^{10} a^{4}+1456 \beta^{6} a^{8}+57344 \beta^{12} a^{2}+\beta^{2} a^{12}+4096 \beta^{14}\right] \\
& T=\frac{\left(96 a^{2} \beta^{4}+24 a^{4} \beta^{2}+a^{6}\right) S^{2}+\left(36 a^{3} \beta^{3}+64 a \beta^{5}+2 a^{5} \beta\right) S+a^{4} \beta^{2}+16 \beta^{6}+16 a^{2} \beta^{4}}{\beta^{2}\left(\left(8 a \beta^{2}+a^{3}\right) S^{2}+\left(4 \beta^{3}+2 a^{2} \beta\right) S+a \beta^{2}\right) a}
\end{aligned}
$$

Agora, como estamos supondo que $b=2,107299 a$, isto vem da definição de $G(a, b)=0$, chamando $k_{0}=2,107299$, e substituindo esse valor de $b$ na expressão que dá $b_{B T}$ obtemos que

$$
a=\frac{\alpha+\sqrt{\alpha^{2}+\beta^{2}}}{k_{0} \beta} .
$$

Substituindo esse valor de a nas expressões do ponto de Bogdanov-Takens obtemos as coordenadas desse ponto dependendo apenas de $\alpha$ e $\beta$. Porém, as expressões são demasiado longas para apresentarmos. Por este motivo, se supormos que temos a seguinte família $Y_{\lambda}(x, y)=(0,1)$, ou seja, $\alpha=0$ e $\beta=1$, poderemos então fazer as contas do ponto de 
Bogdanov-Takens. Ou seja, o ponto de Bogdanov-Takens tem coordenadas

$$
p_{B T}=\left(x_{B T}, y_{B T}, b_{B T}, \lambda_{B T}\right)=(0,5298295,0,35053864,0,475411,-0,4177708) .
$$

Resumimos os resultados deste capítulo na seguinte tabela.

\begin{tabular}{|c|c|c|c|}
\hline Nome & Descrição Filippov & Descrição analítica & $\begin{array}{ll}\text { Explicação } & \text { via } \\
\text { regularização } & \\
\end{array}$ \\
\hline $\begin{array}{l}\mathcal{F}_{1}, \quad \text { Hopf-Sela- } \\
\text { Nó }\end{array}$ & $\begin{array}{l}1 \text { foco repulsor de } X_{\lambda} \text {, } \\
1 \text { ciclo limite de Filip- } \\
\text { pov e uma sela de Fil- } \\
\text { ippov }\end{array}$ & $\begin{array}{l}\alpha>0, \beta>0, a \beta- \\
c \alpha>0, G(a, b, c, d)< \\
0\end{array}$ & $\begin{array}{l}\begin{array}{l}\text { Bifurcação } \\
\text { transição }\end{array} \text { foco-nó, } \\
\text { bifurcação } \\
\text { com vela-nó } \\
\text { atratora }\end{array}$ \\
\hline $\mathcal{F}_{2}$, Sela-Nó & $\begin{array}{l}1 \text { foco repulsor de } X_{\lambda} \\
\text { e uma sela de Filip- } \\
\text { pov }\end{array}$ & $\begin{array}{l}\alpha>0, \beta>0, a \beta- \\
c \alpha>0, G(a, b, c, d)> \\
0\end{array}$ & $\begin{array}{l}\text { Transição foco-nó, bi- } \\
\text { furcação sela-nó com } \\
\text { variedade nodal atra- } \\
\text { tora }\end{array}$ \\
\hline $\begin{array}{l}\mathcal{F}_{4}, \quad \text { Bogdanov- } \\
\text { Takens }\end{array}$ & $\begin{array}{l}1 \text { foco repulsor de } X_{\lambda} \\
\text { e uma sela de Filip- } \\
\text { pov contendo um laço } \\
\text { de sela }\end{array}$ & $\begin{array}{l}\alpha>0, \beta>0, a \beta- \\
c \alpha>0, G(a, b, c, d)= \\
0\end{array}$ & $\begin{array}{ll}\text { Bifurcação } & \text { de } \\
\text { Bogdanov-Takens } & \end{array}$ \\
\hline
\end{tabular}

Tabela 3.1: Tabela dos resultados obtidos 


\section{Capítulo 4}

\section{Bifurcação foco no bordo - parte II}

Neste capítulo daremos continuidade ao estudo das bifurcações dos subcasos de foco no bordo através do método da regularização. Analisaremos os subcasos $\mathcal{F}_{3}, \mathcal{F}_{5}$ e $\mathcal{F}_{6}$.

\subsection{Bifurcação de $\mathcal{F}_{3}$ via regularização}

Agora vamos apresentar a bifurcação que ocorre na FCR de uma FCD pertencente ao terceiro caso.

Teorema 29. Sejam $Z_{\lambda}$ uma FCD do tipo $\mathcal{F}_{3}$. Se

$$
a+d<\min \left\{\frac{(a d-b c) \beta}{a \beta-c \alpha}, 8 \sqrt{3} \beta\right\},
$$

$b \neq b_{0}$, onde $b_{0}$ é dado por (3.37) e $\delta=\Phi(a+d, \beta)$ é dado por (3.33). Então a respectiva família de campos regularizados $Z_{\lambda, R}$ possui uma bifurcação de Hopf em $\lambda_{h}$.

Demonstração. Os cálculos que necessitamos estão feitos nas seções do capítulo anterior e tratam da bifurcação do caso $\mathcal{F}_{1}$. Resta impor as hipóteses iniciais do caso $\mathcal{F}_{3}$.

Os valores da abscissa, ordenada e do parâmetro das singularidades onde o traço da matriz Jacobiana se anula são dados nas equações (3.13) e (3.30), e denotados por $x_{h}, y_{h}$ e $\lambda_{h}$, respectivamente. Para que o determinante Jacobiano calculado em $x_{h}, y_{h}$ e $\lambda_{h}$ seja positivo devemos ter

$$
\frac{\left(1+\Phi^{2}\right)\left(\Phi+\sqrt{1+\Phi^{2}}\right)}{1+\left(\Phi-\sqrt{1+\Phi^{2}}\right)^{2}}>\frac{a \beta-\alpha c}{a d-b c} .
$$

Como $a \beta-\alpha c<0$ e sabendo que o lado esquerdo da desigualdade acima é sempre positivo, então essa desigualdade sempre será satisfeita.

Por fim, o primeiro número de Lyapunov, dado por (3.34), não se modifica e como não há restrições adicionais à $\mathrm{FCD} Z_{\lambda}$, comparado com o caso $\mathcal{F}_{1}$, temos que $l_{1} \neq 0$. Provando 
assim, a existência de uma bifurcação de Hopf no caso $\mathcal{F}_{3}$.

Com relação ao Lema 18, o que muda na região que define $a$ e $d$ é o fato de termos $\frac{a^{2}+b c}{c(a+d)}<\frac{\alpha}{\beta}$ e $a<0$. Portanto, pela prova do Lema 18 temos que a região que procuramos está inteiramente contida no quarto quadrante, implicando que $a<0$ e $d>0$. A próxima figura apresenta um exemplo da região com $b c=-1$. 


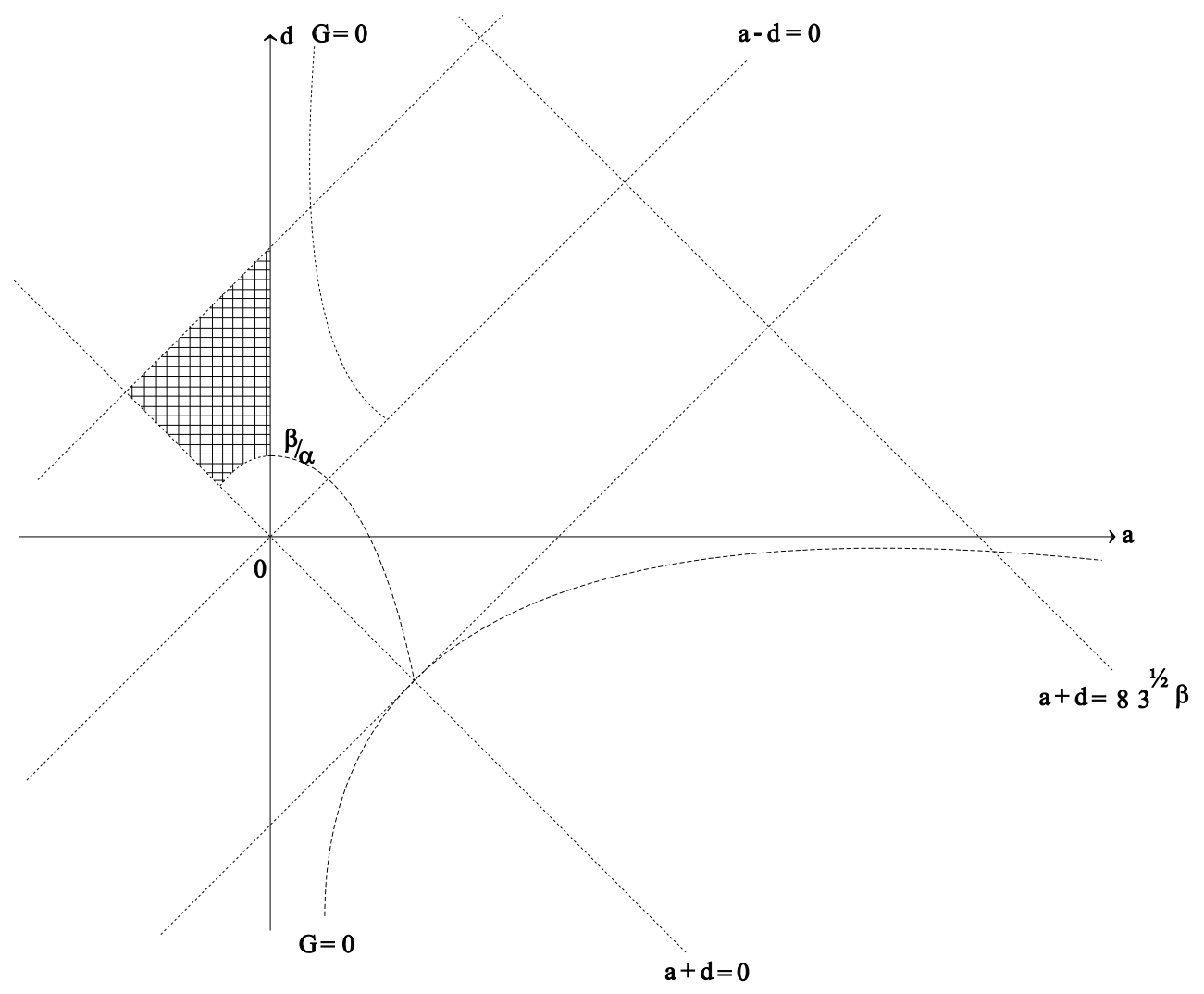

figura 1: região onde valem $a$ e $d$

Assim, não é difícil verificar a seguinte proposição, cuja prova é análoga à Proposição 19.

Proposição 30. Seja $Z_{\lambda}$ uma $F C D$ do tipo $\mathcal{F}_{3}$. Vamos supor que $b \neq b_{0}$, onde $b_{0}$ é dado por (3.37) e $\delta=\Phi(a+d, \beta)$ é dado por (3.33), e vamos considerar que $d F_{4}+\beta F_{7} \neq 0$, onde $F_{4}$ e $F_{7}$ são dados na definição de (3.36). Então, a família regularizada, $Z_{\lambda, R}$, admite um ponto de Hopf cujo primeiro número de Lyapunov é negativo em qualquer um dos seguintes casos

1) se $0<a+d \leq 1,69 \beta$, com $b_{0} \leq 0$ e $b>0$ ou com $b_{0}>0$ e $b>b_{0}$.

2) $\operatorname{sejam~} 1,69 \beta<a+d \leq 8,4 \beta$

2.1) se $\frac{F_{4}}{F_{7}}<-\frac{\beta}{d}$ com $b_{0} \leq 0$ e $b>0$ ou com $b_{0}>0$ e $b>b_{0}$.

2.2) se $\frac{F_{4}}{F_{7}}>-\frac{\beta}{d}$ com $b_{0}>0$ e $0<b<b_{0}$.

3) se $8,4 \beta<a+d<8 \sqrt{3} \beta$ com $b_{0}>0$ e $0<b<b_{0}$. 
Obs.: A curva de Hopf nesse caso é análoga à curva de Hopf do caso $\mathcal{F}_{1}$, no plano $(\varepsilon, \lambda)$.

A próxima figura apresenta o diagrama de bifurcação da regularização de uma família descontínua que pertença ao caso $\mathcal{F}_{3}$. A visualização do diagrama é facilitada pelo fato de ocorrer apenas uma bifurcação do tipo Hopf, que troca a estabilidade do foco, passa de repulsor a atrator. Além disso, o foco atrator passa a nó atrator. 

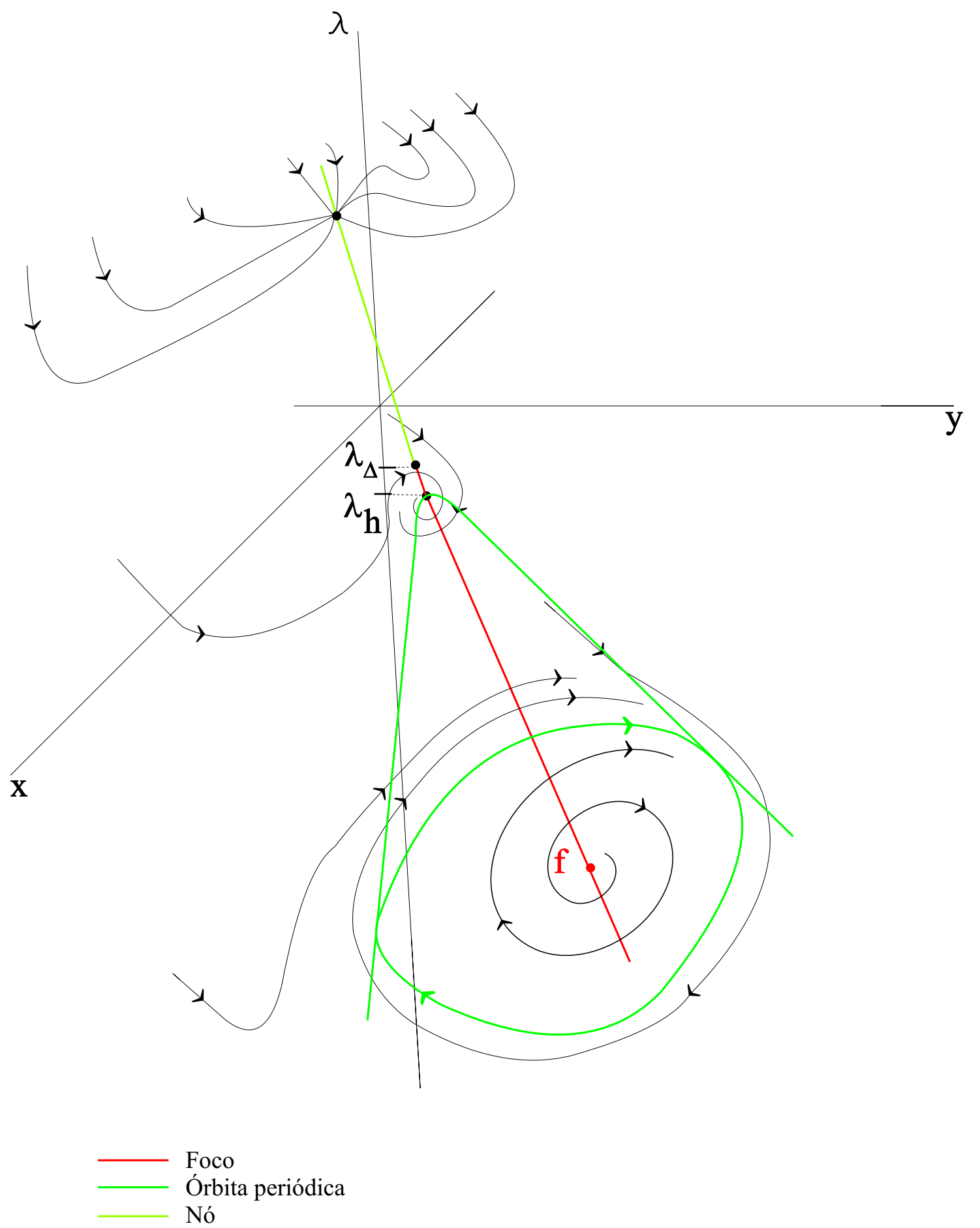

figura 2: Diagrama de bifurcação da família regularizada, referente ao caso $\mathcal{F}_{3}$. 


\subsection{Bifurcação de $\mathcal{F}_{5}$ e $\mathcal{F}_{6}$ via regularização}

Nesta seção analisaremos a família regularizada de uma família descontínua que seja do tipo $\mathcal{F}_{5}$.

Em primeiro lugar, notamos que a FCD a considerar é $Z_{\lambda}=\left(X_{\lambda}, Y_{\lambda}\right)$ onde

$$
\begin{aligned}
& X_{\lambda}(x, y)=(a x+b y+e \lambda, c x+d y+f \lambda) \\
& Y_{\lambda}(x, y)=(\alpha, \beta)
\end{aligned}
$$

onde $a+d>0, c<0, a d-b c>0,(a-d)^{2}+4 b c<0, \alpha<0$ e $\beta<0$.

Para este caso temos que o conjunto de deslizamento é $\mathcal{D}_{Z_{\lambda}}=\left\{(x, 0) \in \mathbb{R}^{2}: x \leq-f \lambda / c\right\}$. Utilizando os cálculos feitos no capítulo anterior, temos que o ponto de tangência de uma órbita que sai do foco com o conjunto de descontinuidade é dado por $p_{t}=(-f \lambda / c, 0)$. Pela prova do lema 11 do capítulo anterior e notando que o caso $\mathcal{F}_{5}$ não tem singularidades de Filippov, ao passo que $\mathcal{F}_{6}$ tem uma sela de Filippov, temos então que o caso $\mathcal{F}_{5}$ é caracterizado por $c \alpha-a \beta>0$ e $\mathcal{F}_{6}$ vale que $c \alpha-a \beta<0$.

Teorema 31. Seja $Z_{\lambda}=\left(X_{\lambda}, Y_{\lambda}\right)$ a FCD que satisfaz as hipóteses do Lema anterior. Sejam $\varepsilon>0$ e $\varphi$ a função de transição. Então valem as seguintes afirmações

a) Se $Z_{\lambda}$ é do tipo $\mathcal{F}_{5}$, então a família regularizada $Z_{\lambda, R}$ possui para valores pequenos e negativos do parâmetro $\lambda$ uma singularidade do tipo foco repulsor, e para valores positivos de $\lambda$ um nó repulsor.

b) Se $Z_{\lambda}$ é do tipo $\mathcal{F}_{6}$, então a família regularizada $Z_{\lambda, R}$ possui uma bifurcação do tipo sela-nó em $y^{*}$, dado por (3.18).

Demonstração. Dado $\varepsilon>0$ e $\varphi$ a função de transição descrita anteriormente, a família regularizada $Z_{\lambda, R}$ que analisaremos é (3.12).

a) Se $Z_{\lambda}$ é do tipo $\mathcal{F}_{5}$ então $a \beta-c \alpha<0$ e isso implica que não vale o Lema 12 . Por outro lado, a expressão que dá os parâmetros das singularidades, dada por (3.13), tem limite, quando $y$ vai a $+\infty$, igual a $-\infty$, e quando $y$ vai a $-\infty$ o seu limite é $+\infty$. Além disso, como a derivada dessa expressão, em função de $y$, só tem pontos críticos quando $c \alpha-a \beta<0$ então temos que para cada $y$ só temos uma singularidade.

O item a) do Lema 13 ainda se aplica nesse caso, ou seja, quando $y=y^{*}$, onde $y^{*}$ é dado por (3.18), o Jacobiano se anula. Assim, temos que nesse ponto $\Delta(y)=\operatorname{tr}\left(D Z_{\lambda, R}\right)^{2}-$ $4 \operatorname{det}\left(D Z_{\lambda, R}\right)>0$. Por outro lado, temos que quando $y$ vai a $+\infty$, o limite de $\Delta$ vale $(a-$ 
$d)^{2}+4 b c<0$. Portanto, existe um ponto $y_{\Delta} \in\left(y^{*}, \infty\right)$ onde $\Delta\left(y_{\Delta}\right)=0$. Isso prova o item a).

b) A prova desse item é uma consequência imediata do Lema 12, do item a) do Lema 13 e da Proposição 15.

Obs.: Na prova do item a) do Teorema anterior verificamos a existência do ponto de transição de foco para nó através do ponto onde o Jacobiano da família regularizada se anula. Como estamos interessados em intervalos pequenos de definição do parâmetro $\lambda$, basta pegar esse intervalo de modo que o ponto $\lambda^{*}$ não pertença a esse intervalo, implicando que não ocorre a existência de uma sela no lugar do foco.

As próximas figuras apresentam os diagramas de bifurcações das regularizações de famílias descontínuas que pertençam aos casos $\mathcal{F}_{5}$ e $\mathcal{F}_{6}$. 


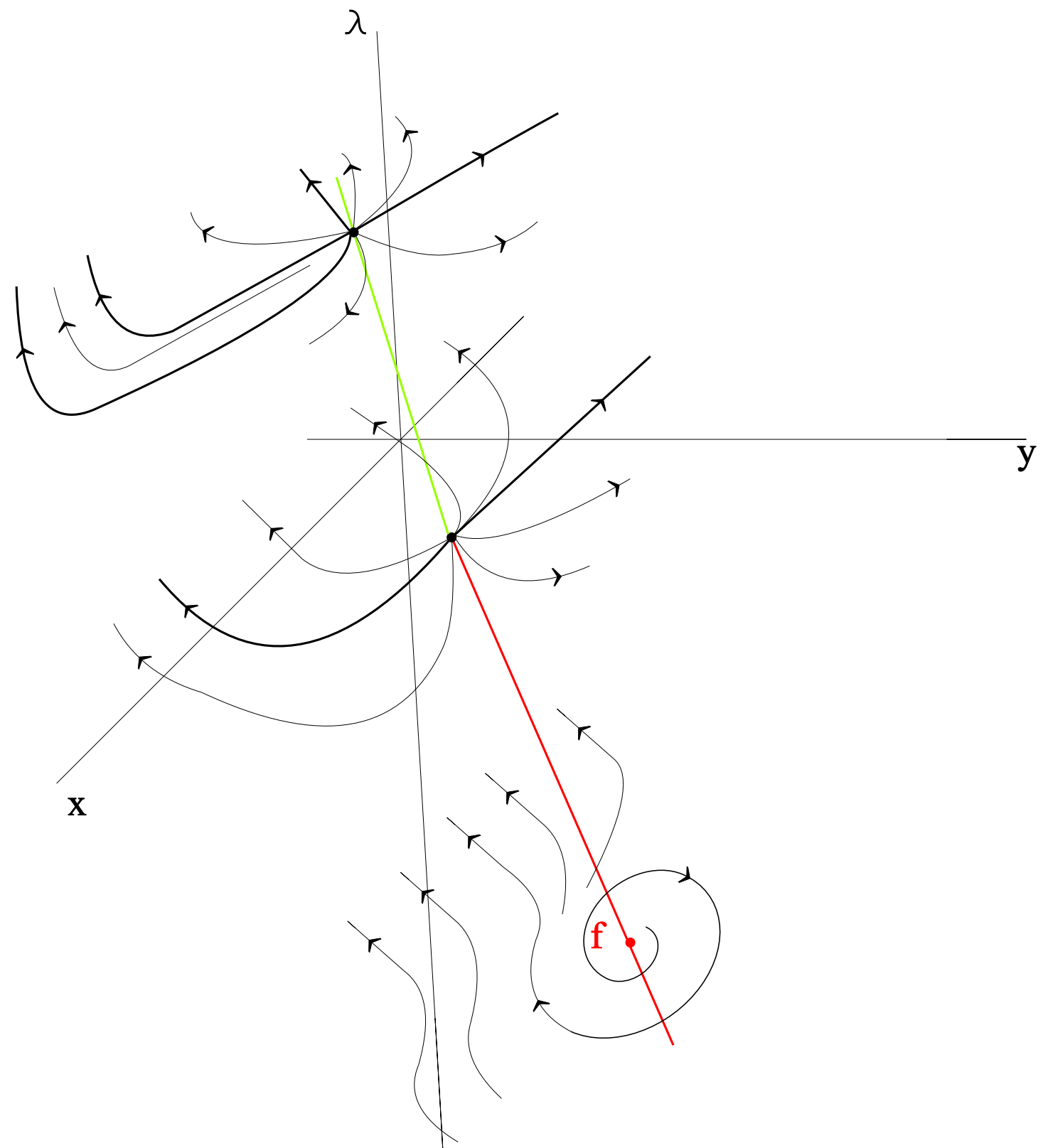

Foco

Órbita periódica

Nó

figura 3: Diagrama de bifurcação de $\mathcal{F}_{5}$ 


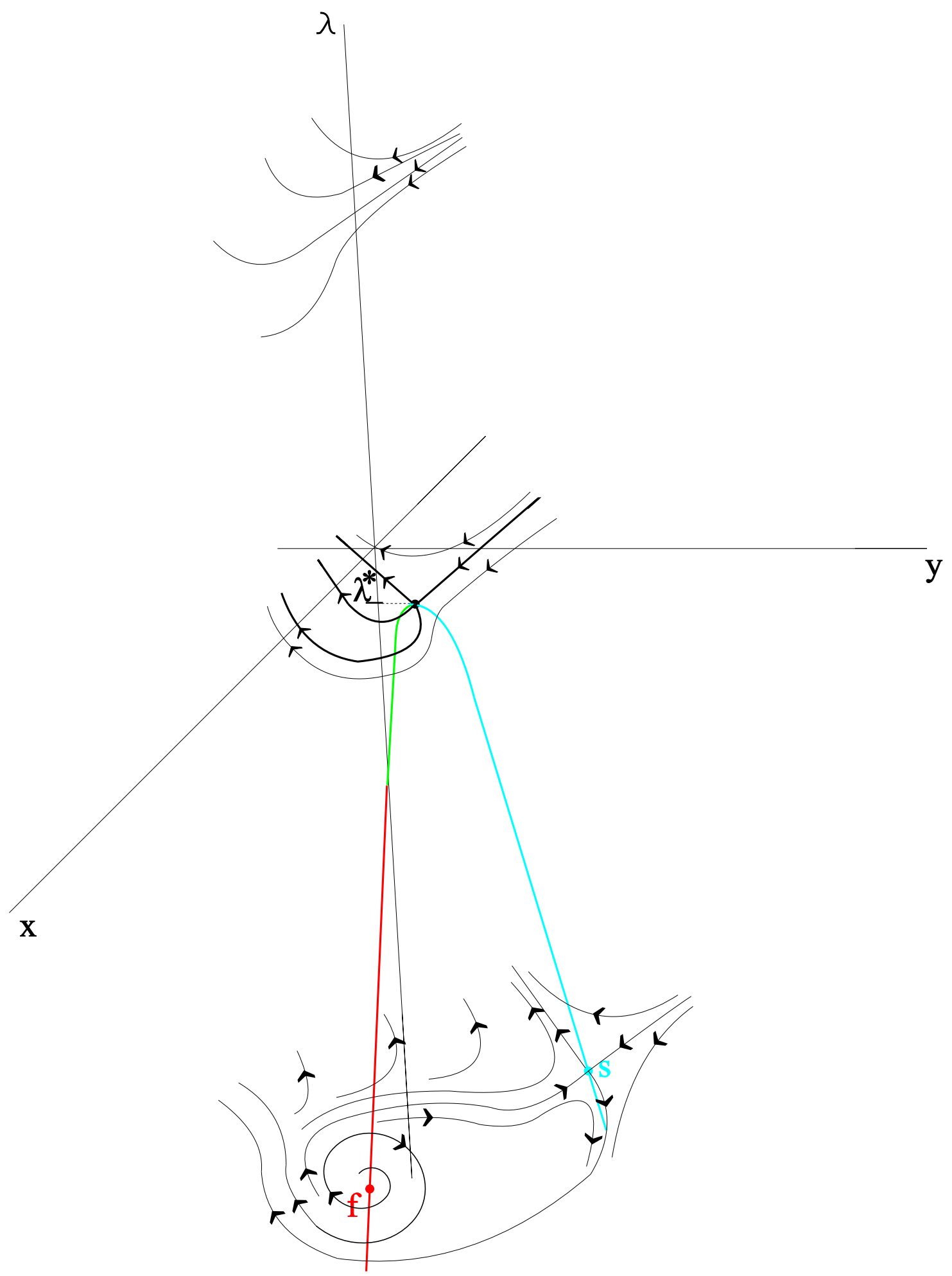

Sela

Foco

Nó

figura 4: Diagrama de bifurcação de $\mathcal{F}_{6}$ 
A próxima tabela contém os resultados obtidos através do método da regularização dos casos de foco no bordo estudados neste capítulo.

\begin{tabular}{|l|l|l|l|}
\hline Nome & Descrição Filippov & Descrição analítica & $\begin{array}{l}\text { Explicação via reg- } \\
\text { ularização }\end{array}$ \\
\hline \hline $\mathcal{F}_{3}$, Hopf & $\begin{array}{l}1 \text { foco repulsor de } X_{\lambda}, \\
1 \text { ciclo limite de Filip- } \\
\text { pov }\end{array}$ & $\begin{array}{l}\alpha>0, \beta>0, a< \\
0, a \beta-c \alpha<0,\end{array}$ & $\begin{array}{l}\text { Bifurcação Hopf, } \\
\text { transição foco-nó }\end{array}$ \\
\hline $\mathcal{F}_{5}$, Foco-Nó & 1 foco repulsor de $X_{\lambda}$ & $\begin{array}{l}\alpha<0, \beta<0, a \beta- \\
c \alpha<0,\end{array}$ & Transição foco-nó \\
\hline $\mathcal{F}_{6}$, Sela-Nó & $\begin{array}{l}1 \text { foco repulsor de } X_{\lambda} \\
\text { e uma sela de Filip- } \\
\text { pov }\end{array}$ & $\begin{array}{l}\alpha<0, \beta<0, a \beta- \\
c \alpha>0,\end{array}$ & $\begin{array}{l}\text { Transição foco-nó e } \\
\text { bifurcação sela-nó }\end{array}$ \\
\hline
\end{tabular}

Tabela 4.1: Tabela dos resultados obtidos 


\section{Capítulo 5}

\section{Bifurcação Sela no bordo}

Neste capítulo vamos estudar as bifurcações de uma família de campos vetoriais descontínuos que sejam dos dois tipo de sela no bordo, descritos na seção 3.1.3 de [KGR], usando o método da regularização.

\subsection{Bifurcação sela no bordo via regularização}

Vamos considerar a família a um parâmetro, $\lambda, Z_{\lambda}=\left(X_{\lambda}, Y_{\lambda}\right)$ de campos vetoriais descontínuos. Vamos supor que $Z_{\lambda}$ está definido no conjunto $M$ do plano $\mathbb{R}^{2}$ e que o conjunto de descontinuidade $D$ é dado por $\{y=0\}$.

Como no caso foco no bordo, em [KGR] há a descrição das bifurcações a um parâmetro que ocorrem na família de CVD que possuem uma singularidade do campo $X_{\lambda}$, para valores negativos e pequenos do parâmetro, que colide com o conjunto de descontinuidade, quando o parâmetro se anula. A diferença para esta caso é que a singularidade é uma sela. Portanto, a família de campos vetoriais descontínuos a ser estudada satisfaz

$$
\begin{aligned}
X_{\lambda}(x, y) & =(a x+b y+e \lambda, c x+d y+f \lambda) \\
Y_{\lambda}(x, y) & =(\alpha, \beta)
\end{aligned}
$$

onde $a, b, c, d, e, f$ são números reais, $\alpha \in \mathbb{R}^{+}$e $\beta \in \mathbb{R}^{+}$. As hipóteses iniciais sobre os coeficientes de $X_{\lambda}$ são que $a d-b c<0$.

Conforme as próximas figuras, a presença da sela em $N$ faz com que o ponto $T$, que é o bordo do conjunto de deslizamento, seja uma tangência quadrática externa de $X_{\lambda}$. Além disso, $T \in\left(p_{e}, p_{i}\right)$ onde $p_{i}$ é o ponto de interseção entre a variedade instável da sela e o conjunto de descontinudiade, e $p_{e}$ o ponto de interseção da variedade estável com $D$. Sejam $D_{d}=\left[p_{i}, \infty\right)$ e $D_{e}=\left(-\infty, p_{i}\right]$. Portanto, o conjunto de descontinuidade $D$ é escrito por 
$D=D_{e} \cup D_{d}$. Assim, usando essas notações definimos abaixo os dois casos que iremos analisar. As figuras 1 e 2 apresentam o diagrama de bifurcação para esses dois casos.

Definição 16. Seja $Z_{\lambda}=\left(X_{\lambda}, Y_{\lambda}\right)$ uma FCD definida em $M$ onde $Y_{\lambda}=(\alpha, \beta) \operatorname{com} \alpha>0$ e $\beta>0$. Dizemos que

a) $Z_{\lambda}$ pertence ao caso $\mathcal{S}_{1}$ se para valores pequenos e negativos do parâmetro possui uma sela $s$ do campo $X_{\lambda}$ em $N$. Além disso, existe um nó de Filippov atrator $n$ no intervalo $D_{d}$. Quando o parâmetro se anula o nó e a sela colidem, e para valores positivos e pequenos do parâmetro esse ponto de colisão vira um ponto de tangência quadrática do campo $X_{\lambda}$.

b) $Z_{\lambda}$ pertence ao caso $\mathcal{S}_{2}$ se para valores pequenos e negativos do parâmetro possui uma sela, $s$, do campo $X_{\lambda}$ em $N$. Além disso, existe um nó de Filippov atrator no intervalo $D_{e}$. Quando o parâmetro se anula o nó e a sela colidem, e para valores positivos e pequenos do parâmetro esse ponto de colisão vira um ponto de tangência quadrática do campo $X_{\lambda}$.

Obs.: Observamos que a família regularizada do terceiro caso descrito em [KGR] não apresenta uma bifurcação do tipo sela-nó, na verdade o que ocorre nessa família é simplesmente que a sela se aproxima do conjunto de descontinuidade quando variamos o parâmetro. Pela perspectiva de $[\mathrm{KGR}]$, a bifurcação que ocorre nesse terceiro caso é a transformação da sela do campo $X_{\lambda}$ para uma sela do campo de Filippov. Por outro lado, pelas regras da regularização, o que ocorre é apenas o deslocamento de uma sela. Por este motivo não consideraremos este caso.

Os casos $\mathcal{S}_{1}$ e $\mathcal{S}_{2}$ são diferenciados pela posição do nó de Filippov com relação ao ponto $p_{i}$. Para o estudo da bifurcação de suas regularizações não precisaremos dessa distinção, ou seja, vamos calcular as bifurcações para um caso que engloba $\mathcal{S}_{1}$ e $\mathcal{S}_{2}$. 


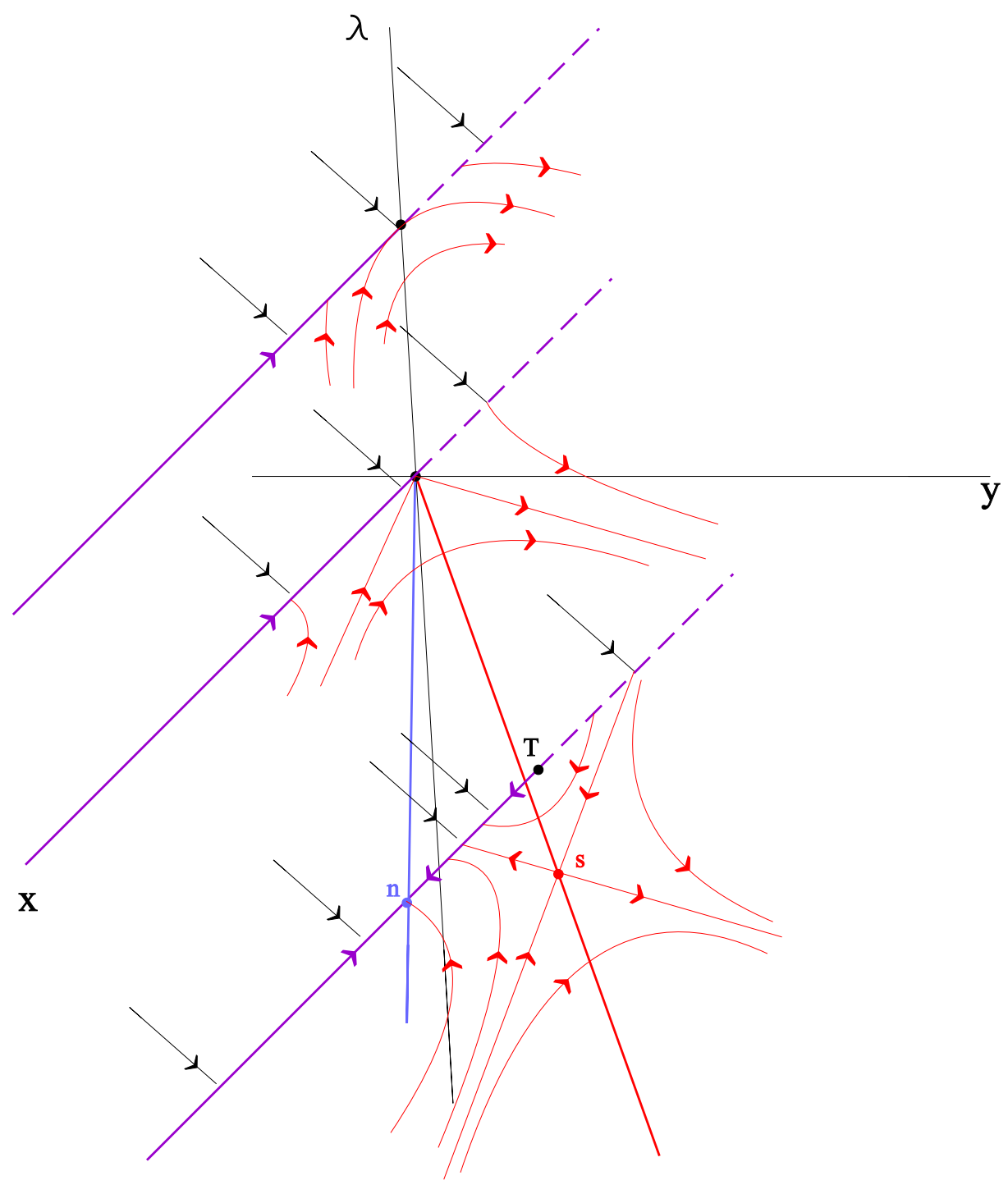

Conjunto de deslizamento

Conjunto de costura

- - Sela

Nó

figura 1: caso $\mathcal{S}_{1}$ 


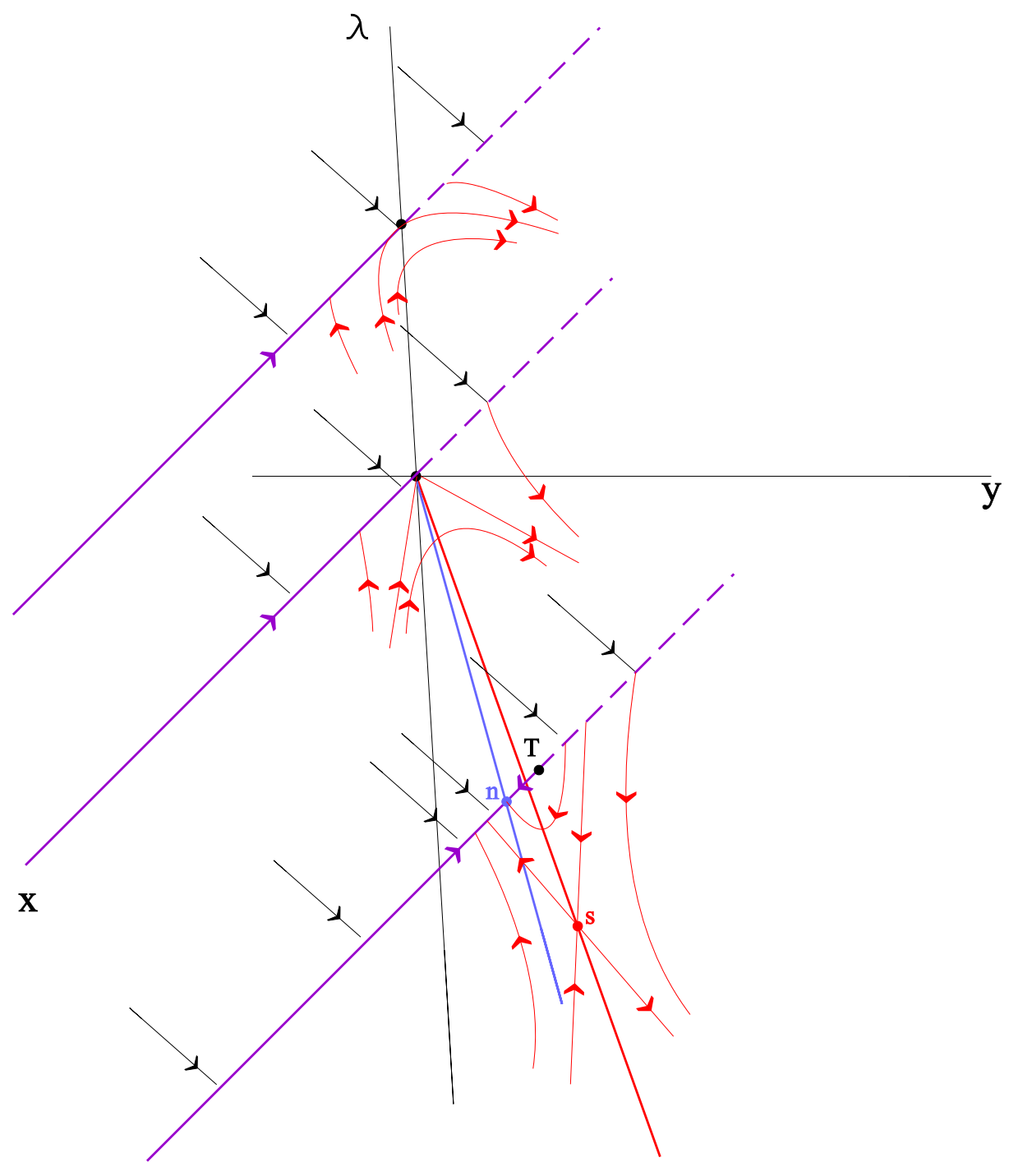

Conjunto de deslizamento

Conjunto de costura

- - Sela

Nó

figura 2: caso $\mathcal{S}_{2}$ 
Para identificar cada um dos casos $\mathcal{S}_{1}$ e $\mathcal{S}_{2}$, devemos verificar a posição da interseção da isóclina que anula a primeira componente do campo $X_{\lambda}$ com o conjunto de descontinuidade. Isto se deve ao fato que esse ponto de interseção é o nó de Filippov. Como dissemos anteriormente, para os nossos propósitos não apresentaremos uma distinção analítica dos dois casos.

Como a primeira componente de $X_{\lambda}$ é $a x+b y+e \lambda$, a reta que anula essa componente é $y=-\frac{a x}{b}-\frac{e \lambda}{b}$. Notamos que se $b=0$ então $a \neq 0$ pois, caso contrário, teríamos que $a d-b c=0$ contrariando a hipótese inicial. Essa reta intersecta $D$ em um único ponto que é dado por $-\frac{e \lambda}{a}$, assumindo que $a \neq 0$. Portanto, o nó de Filippov é dado por

$$
n=\left(-\frac{e \lambda}{a}, 0\right)
$$

Por outro lado, temos que o ponto $T$ de tangência quadrática é a interseção da isóclina que anula a segunda componente de $X_{\lambda} \operatorname{com} D$. Assim, fazendo cálculos análogos aos acima, e supondo que $c \neq 0$, obtemos

$$
T=\left(-\frac{f \lambda}{c}, 0\right) .
$$

Notamos que o ponto $T$ é o ponto do bordo do conjunto de deslizamento. Para ver isto notamos que o conjunto de deslizamento é dado por $X F(x, y) Y F(x, y) \leq 0$, mas $X F(x, y) Y F(x, y)=(c x+f \lambda) \beta \leq 0$ se $c x \leq-f \lambda$. Como o conjunto de deslizamento deve ficar no intervalo $[T,+\infty)$, devemos supor que $c<0$. Assim, o conjunto de deslizamento é dado por $\left\{x \in \mathbb{R}: x \geq-\frac{f \lambda}{c}\right\}$.

Além das hipóteses acima, $a \neq 0, b \neq 0$ e $c<0$, temos a hipótese que garante que a sela do campo $X_{\lambda}$ fique em $N$ para valores negativos de $\lambda$. Como vimos no capítulo 3 , as singularidades de $X_{\lambda}$ são dadas por (3.3), e como $a d-b c<0$ para que a ordenada da sela, dada por $y=\frac{(c e-a f) \lambda}{a d-b c}$, seja positiva devemos ter $c e-a f>0$.

Pelos diagramas de bifurcação dos dois casos da sela no bordo, devemos ter que

$$
-\frac{f \lambda}{c}<-\frac{e \lambda}{a} .
$$

Dessa desigualdade e do fato que $c e-a f>0$ temos que $a$ e $c$ teem o mesmo sinal, donde tiramos que $a<0$.

Para descobrir a última hipótese que devemos impor, vamos novamente recorrer ao capítulo 3 donde temos que as singularidades do campo de Filippov $F_{Z_{\lambda}}$ são da forma $\left(x_{F}, 0\right)$ 
onde $x_{F}=\frac{(e \beta-f \alpha) \lambda}{c \alpha-a \beta}$. Como a singularidade do campo de Filippov está no conjunto de deslizamento, temos que $\frac{(e \beta-f \alpha) \lambda}{c \alpha-a \beta}>-\frac{f \lambda}{c}$, agora lembrando que $\lambda$ e $c$ são ambos negativos chegamos a

$$
\frac{c e \beta-c f \alpha}{c \alpha-a \beta}>-f
$$

Portanto, temos dois casos a considerar, dados de acordo com o sinal de $c \alpha-a \beta$. Vamos supor que $c \alpha-a \beta<0$ então (5.4) se resume a

$$
c e \beta-c f \alpha<-c f \alpha+a f \beta
$$

eliminando os termos iguais e lembrando que $\beta>0$ obtemos $c e<a f$, o que contradiz a hipótese de termos $c e-a f>0$. Portanto, devemos ter que $c \alpha-a \beta>0$.

Resumindo, nossas hipóteses iniciais para uma FCD que pertença aos casos $\mathcal{S}_{1}$ ou $\mathcal{S}_{2}$ são $\alpha>0, \beta>0, a<0, c<0, a d-b c<0,(a-d)^{2}+4 b c>0, c e-a f>0$ e $c \alpha-a \beta>0$.

A regularização a ser feita usa como função de transição a função definida no capítulo 3 . Assim, as etapas da regularização e da mudança de variáveis e reescalonamento do parâmetro feitas no capítulo 3 continuam valendo nesse capítulo, ou seja, a expressão para a família regularizada dos casos $\mathcal{S}_{1}$ e $\mathcal{S}_{2}$ é dada por (3.12). Portanto, os cálculos das singularidades, determinante e traço da matriz Jacobiana continuam sendo os mesmos obtidos no capítulo 3.

Com essas hipóteses, o Lema 12 que determina, entre outras coisas, o parâmetro $\lambda^{*}$ associado à bifurcação de sela-nó é válido para esse caso, sendo que sua prova é análoga à do Lema 12. Por outro lado, o item a) do Lema 13 tem uma modificação neste caso, mas sua prova é feita de forma análoga.

Lema 32. Seja $Z_{\lambda}=\left(X_{\lambda}, Y_{\lambda}\right)$ uma FCD a 1-parâmetro que pertença aos casos $\mathcal{S}_{1}$ ou $\mathcal{S}_{2}$. Se $y^{*}$ é dado por (3.18), então para valores de $y$ estritamente menores que $y^{*}$ as singularidades são nós, em $y^{*}$ o determinante Jacobiano da FCR, dado por (3.16), se anula e se $y>y^{*}$ as singularidades são selas.

A Proposição 15 é provada de forma análoga à do capítulo 3, portanto temos o seguinte resultado.

Teorema 33. Seja $Z_{\lambda}$ uma $F C D$ do tipo $\mathcal{S}_{1}$ ou $\mathcal{S}_{2}$. Então, a respectiva família regularizada $Z_{\lambda, R}$ possui uma bifurcação do tipo sela-nó quando o parâmetro vale $\lambda^{*}$. 
As próximas figuras apresentam o diagrama de bifurcação da regularização de uma família descontínua que pertença aos casos $\mathcal{S}_{1}$ e $\mathcal{S}_{2}$, respectivamente. 


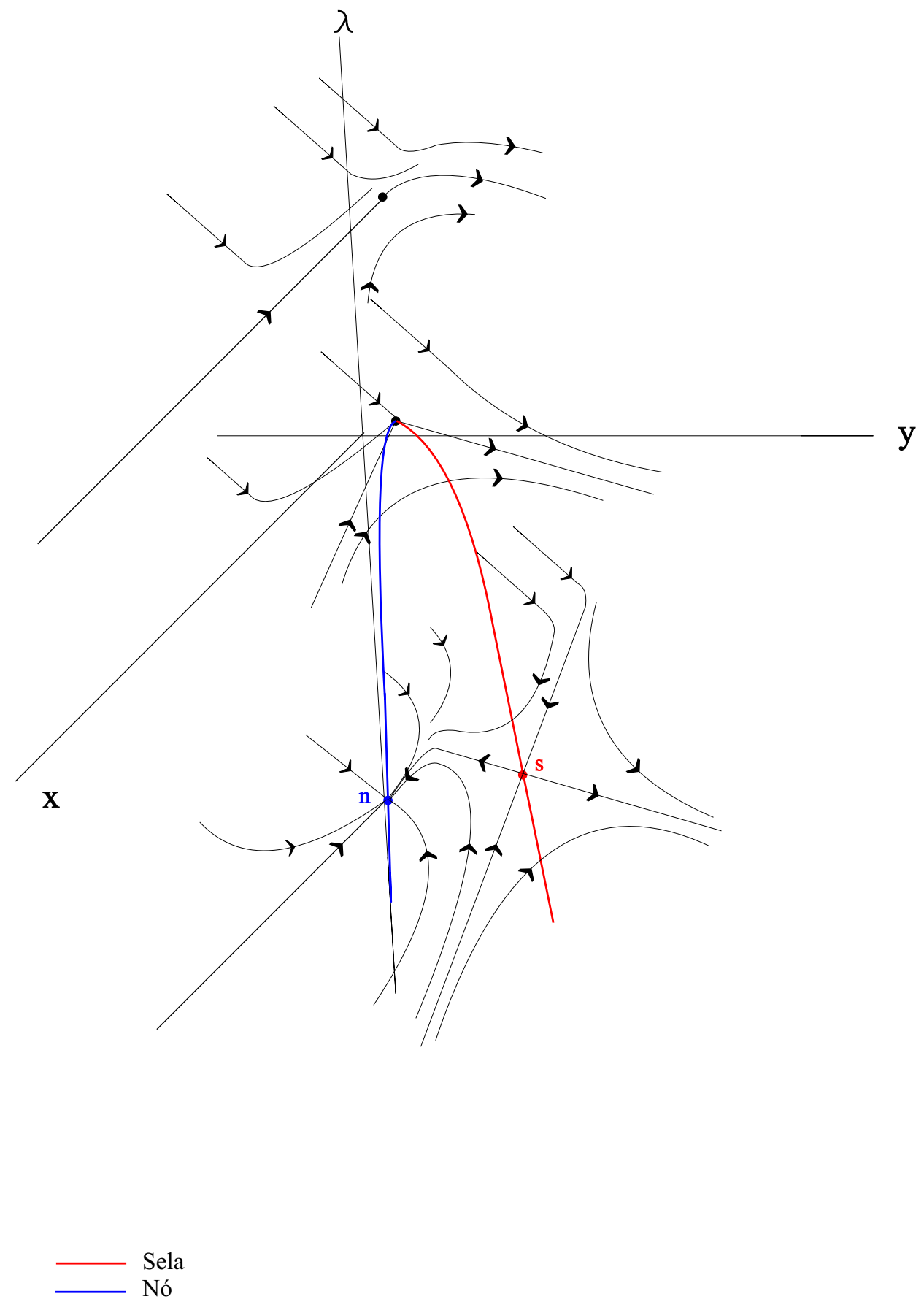

figura 3: regularização do caso $\mathcal{S}_{1}$ 


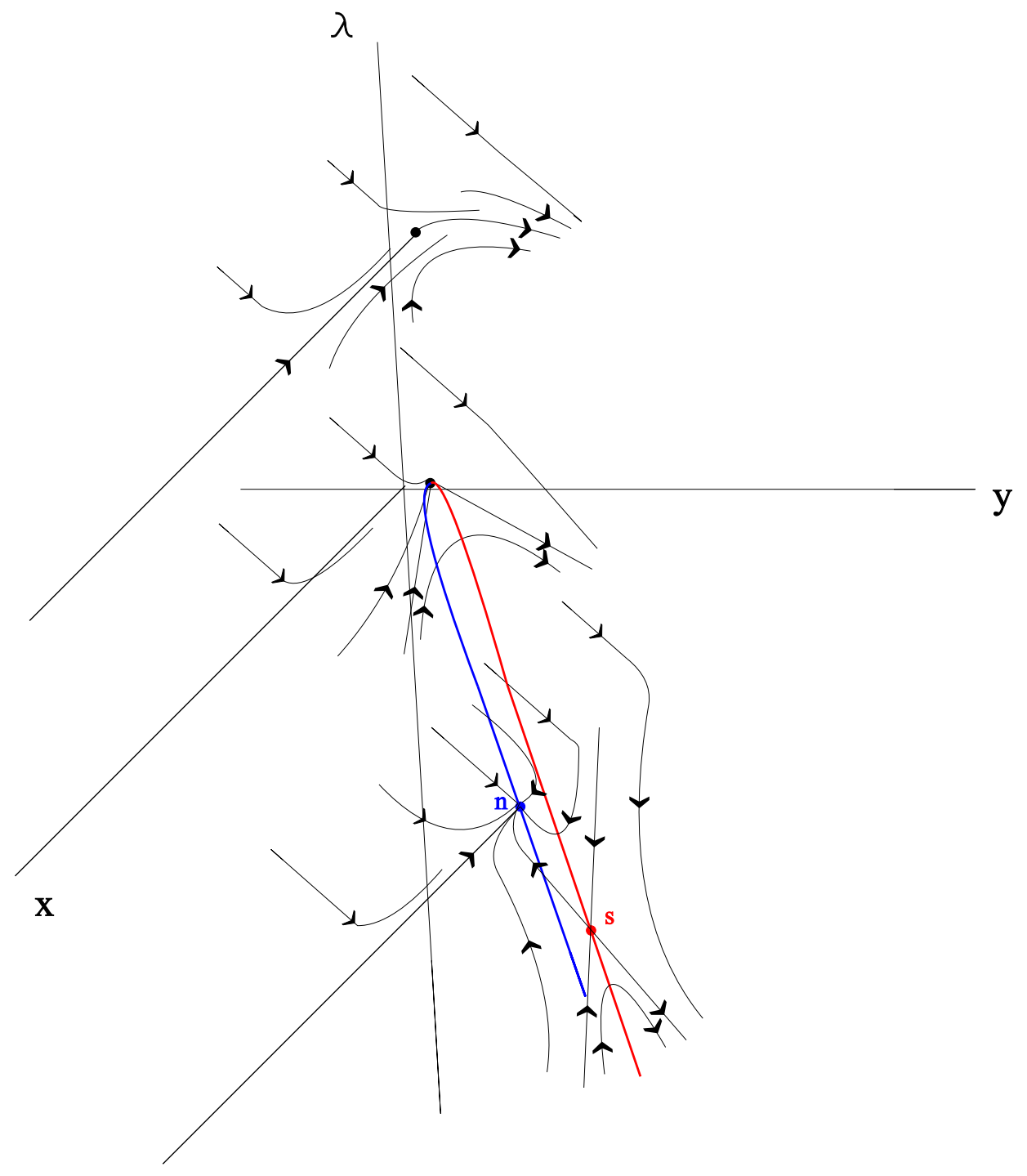

$\begin{array}{ll}\longrightarrow & \text { Sela } \\ & \text { Nó }\end{array}$

figura 4: regularização do caso $\mathcal{S}_{2}$ 
A próxima tabela contém os resultados obtidos através do método da regularização dos casos de foco no bordo estudados neste capítulo.

\begin{tabular}{|l|l|l|}
\hline Nome & Descrição Filippov & $\begin{array}{l}\text { Explicação via } \\
\text { regularização }\end{array}$ \\
\hline \hline $\mathcal{S}_{1}$, Sela-nó & $\begin{array}{l}\text { uma sela de } X_{\lambda}, 1 \text { nó } \\
\text { atrator de Filippov }\end{array}$ & Bifurcação sela-nó \\
\hline $\mathcal{S}_{2}$, Sela-Nó & $\begin{array}{l}\text { uma sela de } X_{\lambda}, 1 \text { nó } \\
\text { atrator de Filippov }\end{array}$ & Bifurcação sela-nó \\
\hline
\end{tabular}

Tabela 5.1: Tabela dos resultados obtidos 


\section{Capítulo 6}

\section{Bifurcação nó no bordo}

Neste capítulo vamos apresentar o estudo da bifurcação da regularização de uma família a um parâmetro descontínua que tem um nó atrator em $N$ que colide com o conjunto de descontinuidade quando o parâmetro se anula. Este caso está descrito na seção 3.1.2 de [KGR].

\subsection{Bifurcação nó no bordo via regularização}

Neste capítulo vamos considerar a família $Z_{\lambda}=\left(X_{\lambda}, Y_{\lambda}\right)$ de campos vetoriais descontínuos

a um parâmetro, $\lambda$. Vamos supor que $Z_{\lambda}$ está definido no conjunto $M$ do plano $\mathbb{R}^{2}$ e que o conjunto de descontinuidade $D$ é dado por $\{y=0\}$.

A família de campos vetoriais a serem estudados são

$$
\begin{aligned}
& X_{\lambda}(x, y)=(a x+b y+e \lambda, c x+d y+f \lambda) \\
& Y_{\lambda}(x, y)=(\alpha, \beta)
\end{aligned}
$$

onde $a, b, c, d, e, f$ são números reais, $\alpha \in \mathbb{R}^{+}$e $\beta \in \mathbb{R}^{-}$. As hipóteses iniciais sobre os coeficientes de $X_{\lambda}$ são que $a d-b c>0,(a-d)^{2}+4 b c>0$ e $a+d<0$.

No artigo $[\mathrm{KGR}]$ existem dois casos para o nó no bordo. No primeiro caso ocorre apenas uma mudança do nó com a variação do parâmetro, no sentido que o nó que era uma singularidade do campo $X_{\lambda}$, para valores negativos e pequenos do parâmetro, vira um nó atrator de Filippov para valores positivos e pequenos do parâmetro. Portanto, pela regras da regularização, este caso não possui nenhuma bifurcação, o que ocorre na família regularizada é apenas um deslocamento do nó. No segundo caso, além do nó atrator de $X_{\lambda}$, para valores pequenos e negativos do parâmetro, há a presença de uma sela de Filippov, e a sua regularização é mais interessante. Portanto, iremos tratar apenas do segundo caso que iremos denotar simplesmente por $\mathcal{N}$. 
A diferença deste caso com relação aos estudados nos capítulos anteriores, é que enquanto nos outros estávamos supondo que $\beta>0$ nesse estaremos supondo que $\beta<0$. E é essa a distinção entre os dois casos de nó no bordo descrito em [KGR], ou seja, se $\beta>0$ então estamos tratando do primeiro caso e se for negativo estamos considerando o caso $\mathcal{N}$.

A definição do caso a ser analisado é a seguinte.

Definição 17. Seja $Z_{\lambda}=\left(X_{\lambda}, Y_{\lambda}\right)$ uma FCD definida em $M$ onde $Y_{\lambda}=(\alpha, \beta)$ com $\alpha>0$ e $\beta<0$. Dizemos que $Z_{\lambda}$ pertence ao caso $\mathcal{N}$ se para valores pequenos e negativos do parâmetro possui um nó atrator, $n$, do campo $X_{\lambda}$ em $N$. Além disso, existe uma sela de Filippov, $s$, no conjunto de descontinuidade e um ponto $T$ de tangência quadrática interna com $D$ de uma órbita que vai ao nó. Quando o parâmetro se anula o nó, a sela e o ponto $T$ colidem na origem, e para valores positivos e pequenos do parâmetro esse ponto de colisão vira um ponto de tangência quadrática externa do campo $X_{\lambda}$.

A próxima figura apresenta o diagrama de bifurcação do caso nó no bordo. 


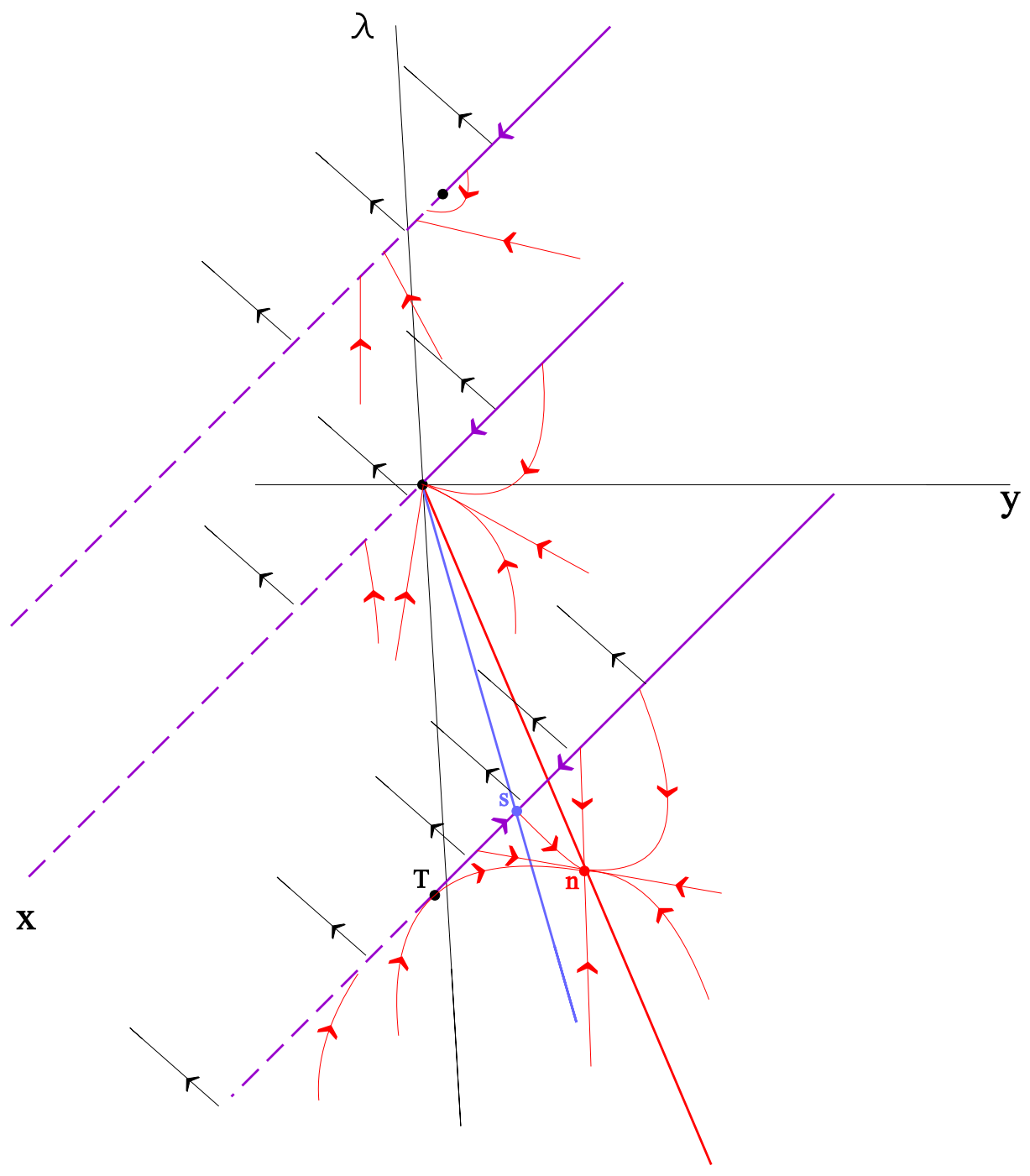

Conjunto de deslizamento

Conjunto de costura

- - Có

Sela

figura 1: diagrama de bifurcação do caso $\mathcal{N}$ 
Agora que temos a figura geral que deve apresentar esse caso, vamos analisá-lo para descobrir as hipóteses a serem cumpridas. Para que a singularidade de $X_{\lambda}$ fique em $N$ para valores negativos do parâmetro, devemos ter que $c e-a f<0$, uma vez que $a d-b c>0$.

Como no caso da sela no bordo, supondo $a \neq 0$ e $c \neq 0$, os pontos $-\frac{f \lambda}{c}$ e $-\frac{e \lambda}{a}$ são de grande importância para este caso, o primeiro é exatamente onde $y^{\prime}=0$ que é onde ocorre a tangência quadrática de uma órbita que vai até o nó com o conjunto de descontinuidade. Assim, $T=\left(-\frac{f \lambda}{c}, 0\right)$ e este é o ponto de separação entre os conjuntos de costura e deslizamento. Por outro lado, o ponto onde $x^{\prime}=0$ é exatamente a sela de Filippov e é dado por $s=\left(-\frac{e \lambda}{a}, 0\right)$.

Falamos anteriormente que o ponto $T$ é a fronteira do conjunto de deslizamento. Mas para que isso faça sentido, e como $\beta<0$, então devemos ter que $c<0$ pois o conjunto de deslizamento é dado por $\left\{x \in \mathbb{R}: x \leq-\frac{f \lambda}{c}\right\}$.

Analisando o diagrama de bifurcação notamos que a abscissa de $T$ deve ser maior que a abscissa de $s$. Assim, devemos ter

$$
-\frac{e \lambda}{a}<-\frac{f \lambda}{c}
$$

Juntando isso ao fato que $c<0$ e $c e-a f<0$, devemos finalmente ter que $a<0$. De fato, como $\lambda<0$ da desigualdade acima temos que $\frac{e}{a}<\frac{f}{c}$ e sendo $c<0$ resulta que $\frac{c e}{a}>f$. Portanto, para que tenhamos ce $-a f<0$ devemos obrigatoriamente ter que $a<0$.

A singularidade do campo de Filippov, como visto nos capítulos anteriores, é dada por $\left(\frac{(e \beta-f \alpha) \lambda}{c \alpha-a \beta}, 0\right)$. Assim, para que essa singularidade pertença ao conjunto de deslizamento devemos ter que

$$
\frac{(e \beta-f \alpha) \lambda}{c \alpha-a \beta}<-\frac{f \lambda}{c}
$$

Agora, lembrando que $\lambda<0$ e $c<0$ obtemos

$$
\frac{c e \beta-f c \alpha}{c \alpha-a \beta}<-f
$$

Vamos supor que $c \alpha-a \beta>0$ então a desigualdade (6.2) é reescrita como $(c e-a f) \beta>0$, contradizendo o fato de termos ce $-a f<0$, uma vez que $\beta<0$. Portanto, temos que $c \alpha-a \beta<0$.

Do que vimos até agora, uma FCD pertence ao caso $\mathcal{N}$ se satisfizer $\alpha>0, \beta<0$, $a<0, c<0, a d-b c>0, a+d<0,(a-d)^{2}+4 b c>0, c e-a f<0$ e $c \alpha-a \beta>0$.

Essas hipótese garantem a validade do Lema 12, que garante a existência de duas sin- 
gularidades e ainda dá o valor do parâmetro onde ocorre a bifurcação, para o caso nó no bordo. Podemos dizer o mesmo sobre o item a) do Lema 13, e a Proposição 15 do capítulo 3 fica inalterada, ou seja, nas hipótese acima deste caso ainda vale esse resultado. Assim, resumimos os cálculos acima no seguinte teorema.

Teorema 34. Seja $Z_{\lambda}$ uma $F C D$ do tipo $\mathcal{N}$. Então, a respectiva família regularizada $Z_{\lambda, R}$ possui uma bifurcação do tipo sela-nó quando o parâmetro vale $\lambda^{*}$.

A próxima figura apresenta o diagrama de bifurcação da regularização de uma família de campos vetoriais descontínua que pertença ao caso $\mathcal{N}$. 


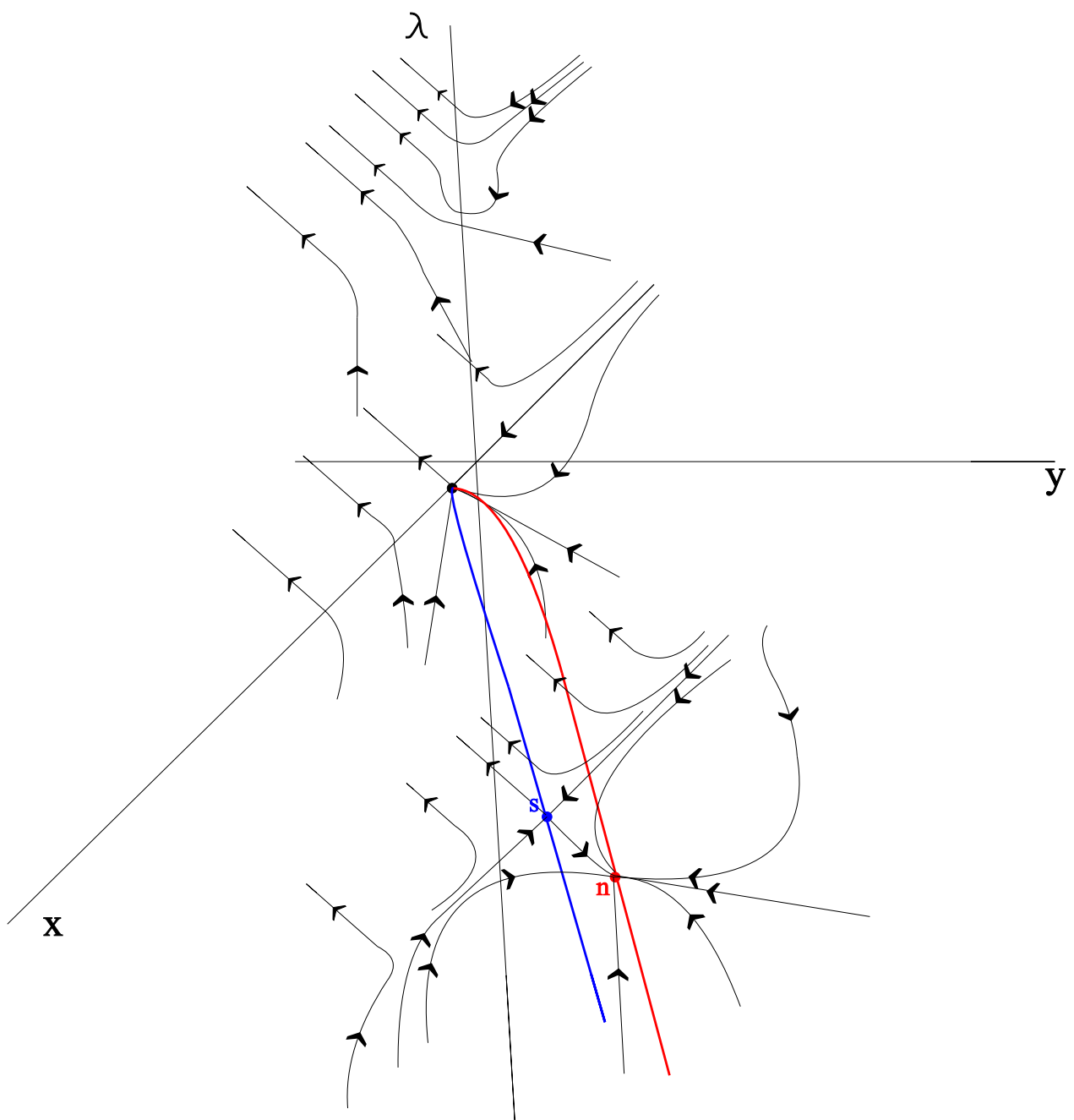

Nó

figura 2: diagrama de bifurcação da regularização do caso $\mathcal{N}$ 


\section{Capítulo 7}

\section{Bifurcação tangência dupla externa}

Neste capítulo estudaremos a regularização de uma família de campos vetoriais descontínuos que tenha uma tangência externa com o conjunto de descontinuidade para a família definida em $N$ e outra tangência com $D$ da família definida em $S$. Estudaremos apenas o segundo caso da seção 3.2.4 de [KGR]. A regularização apresenta uma bifurcação de Hopf donde concluímos que a respectiva família descontínua possui uma bifurcação de Hopf que, no citado artigo, os autores chamam de "bifurcação pseudo-Hopf supercrítica". Além disso, notamos que o primeiro número de Lyapunov para a família regularizada tem o mesmo sinal que o número $V$, que faz o mesmo papel que o primeiro número de Lyapunov para as famílias descontínuas, definido no artigo [CGP].

\subsection{Bifurcação tangência dupla externa via regularização}

A seção 3.2.4 de [KGR] possui dois casos onde ocorre uma bifurcação de codimensão 1 de famílias descontínuas que possuam uma colisão entre duas tangências quadráticas externas. No primeiro caso o ponto de tangência de $X_{\lambda}$ com $D$ é repulsor, visto como uma singularidade do campo de Filippov, e o ponto de tangência de $Y_{\lambda}$ é atrator no mesmo sentido, para valores pequenos e negativos do parâmetro. Além disso, a abscissa do ponto de tangência de $X_{\lambda}$ é maior que a abscissa do ponto de tangência de $Y_{\lambda}$ para valores negativos de $\lambda$. A colisão ocorre quando $\lambda=0$, e para valores positivos e pequenos do parâmetro o ponto de tangência de $Y_{\lambda}$ fica menor que o de $X_{\lambda}$, e trocam as características dos pontos, ou seja, o ponto de tangência de $X_{\lambda}$ vira atrator e o de $Y_{\lambda}$ repulsor. A próxima figura exemplifica o que explicamos através do diagrama de bifurcação deste caso. Utilizando as regras de regularização, veja $[\mathrm{M}]$, $[\mathrm{SM}]$, $[\mathrm{ST}]$ ou o capítulo dois desta tese, obtemos que a família regularizada deste caso não possui singularidades visto que a regularização de uma tangência não gera singularidades, ou seja, a regularização deste caso gera uma família de campos vetoriais do tipo fluxo tubular, para valores pequenos do parâmetro. 


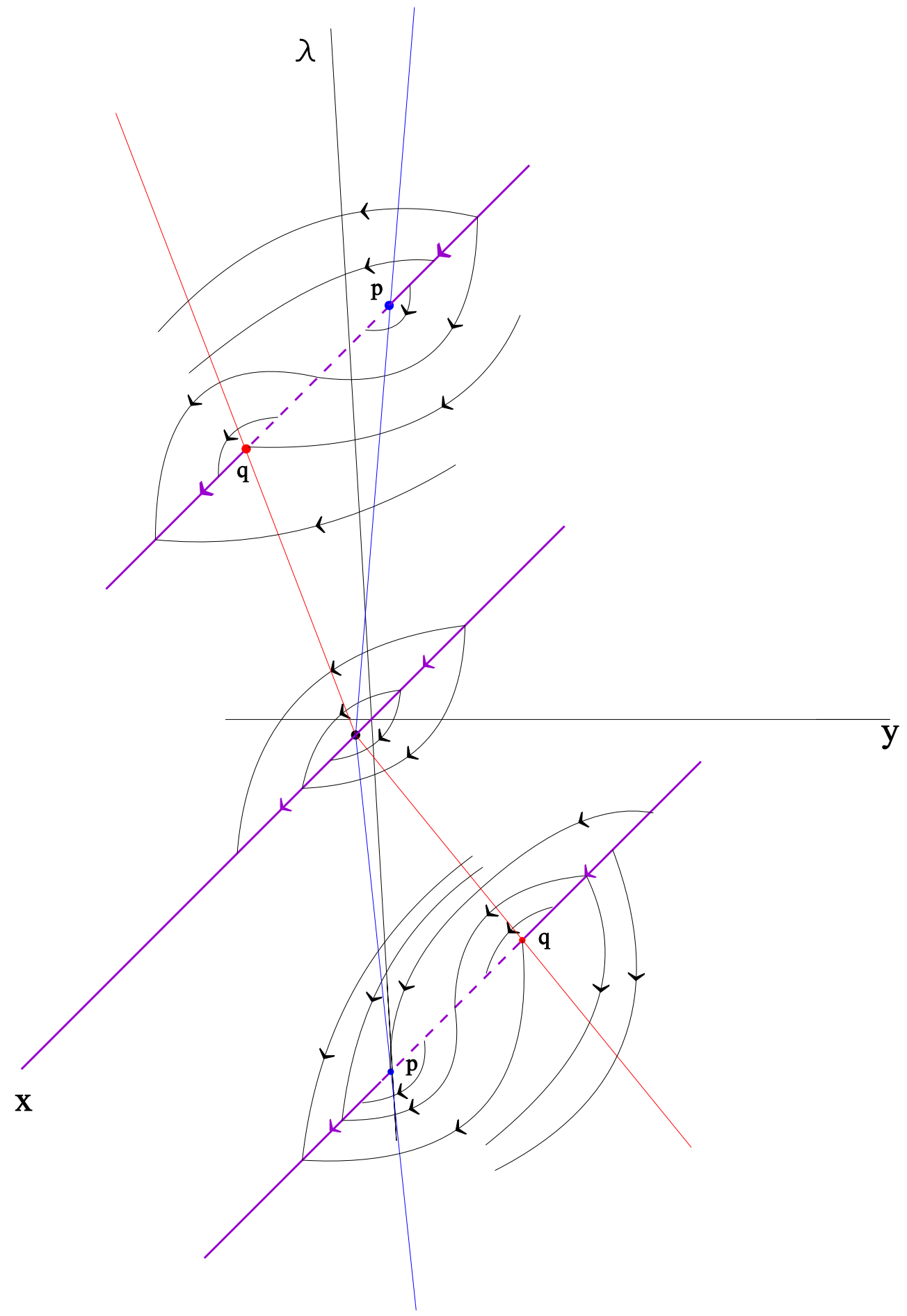

Conjunto de deslizamento

- - - Conjunto de costura

— Ponto de tangência de $\mathrm{X} \lambda$

Ponto de tangência de $\mathrm{Y} \lambda$

figura 1: diagrama de bifurcação do primeiro caso 
O segundo caso é o mais interessante por tratar de uma bifurcação de Hopf para o caso descontínuo, o que os autores de [KGR] chamam de bifurcação pseudo-Hopf supercrítica. Esse caso será definido logo abaixo, e será denotado por $\mathcal{T}_{1}$. As diferenças entre esses dois casos, apresentados no artigo citado, são duas: a primeira se dá na ordem dos pontos de tangência no conjunto de descontinuidade, ou seja, levando em consideração a ordenação da reta real, e a segunda diferença está no sentido das trajetórias da família $X_{\lambda}$. Assim, enquanto os pontos de tangência do primeiro caso têm a ordenação descrita acima, no segundo caso a ordenação dos pontos de tangência é inversa à do primeiro caso, além disso, no segundo caso o sentido das órbitas de $X_{\lambda}$ é o inverso do primeiro caso. Vamos definir o segundo caso.

Definição 18. Seja $Z_{\lambda}=\left(X_{\lambda}, Y_{\lambda}\right)$ uma FCD a um parâmetro definida em $M$. Dizemos que $Z_{\lambda}$ pertence ao caso $\mathcal{T}_{1}$ se para valores negativos e pequenos do parâmetro existirem duas tangências quadráticas externas distintas, uma correspondente ao campo $X_{\lambda}$ com o conjunto de descontinuidade que denotaremos por $p$ e outra do campo $Y_{\lambda}$ com $D$ que denotaremos por $q$. O ponto $p$ deve ser estritamente menor que $q$, e entre esses dois pontos existe um nó atrator de Filippov, que denotaremos por $n_{1}$. Ocorre a colisão de $p$ com $q$ na origem quando $\lambda$ se anula, e para valores positivos e pequenos do parâmetro existe uma órbita periódica atratora tendo no seu interior as duas tangências quadráticas externas, $p$ e $q$, tais que $p$ é maior que $q$. Além disso, existe um nó repulsor de Filippov, denotado por $n_{2}$, entre esses pontos de tangência.

Obs.: Na definição acima fica implícita a dependência dos pontos de tangência quadrática, $p$ e $q$, com relação ao parâmetro $\lambda$. Não colocamos essa dependência nos símbolos dos pontos para simplificar a notação.

A próxima figura apresenta o diagrama de bifurcação de uma família de campos vetoriais descontínuos do tipo $\mathcal{T}_{1}$. 


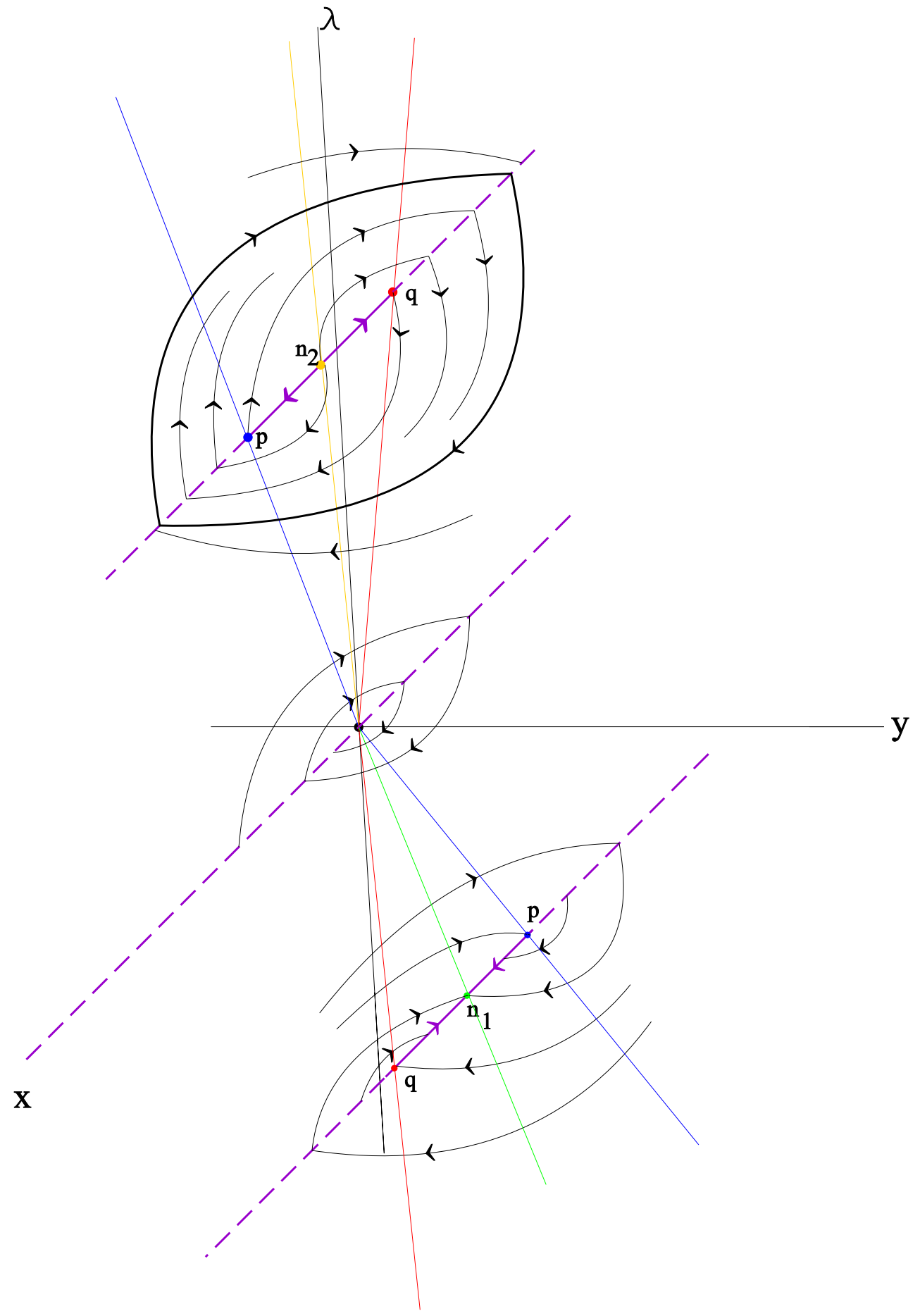

Conjunto de deslizamento

- - - Conjunto de costura

— Ponto de tangência de $\mathrm{X} \lambda$

Ponto de tangência de $\mathrm{Y} \lambda$

Foco atrator

Foco repulsor

figura 2: diagrama de bifurcação do caso $\mathcal{T}_{1}$ 
O aparecimento de ciclo limite a partir de um foco, em uma família a um parâmetro de campos vetoriais regulares, está associada à bifurcação de Hopf. A estabilidade do ponto de Hopf é calculada através do primeiro número de Lyapunov, essa estabilidade será repassada ao ciclo limite. Assim, se o primeiro número de Lyapunov for nulo não aparece ciclo limite hiperbólico, se for negativo o ciclo limite é atrator, e se for positivo será repulsor. Para o caso onde a família de campos vetoriais é descontínua, existe uma teoria análoga ao do caso regular para o cálculo da estabilidade do ponto de Hopf. Essa teoria se resume assim, considerando uma família descontínua com duas tangências que colidem na origem quando o parâmetro se anula, é a estabilidade desse ponto de tangência dupla que dará a existência ou não do ciclo limite. Para o cálculo dessa estabilidade, precisamos de um número que faça o papel equivalente ao primeiro número de Lyapunov. Vamos apresentar o método de CollGasull-Prohens para o cálculo desse número que dará a estabilidade do ponto de tangência dupla.

Aparentemente um dos primeiros a tratar o caso das duas tangências quadráticas externas foi Gubar em 1971, veja [G]. Filippov, em [F], também apresenta o método de estudo da bifurcação de uma família descontínua de campos vetoriais que tenham duas tangências quadráticas externas. Tanto Gubar quanto Filippov chamam o ponto de colisão entre as tangências por "fusão de focos" ou "foco fundido", além desses nomes podemos chamá-lo de ponto de tangência dupla. Tal estudo se baseia na análise da aplicação de primeiro retorno, sendo o conjunto de descontinuidade a seção transversal, quando o parâmetro se anula, ou seja, quando as duas tangências colidem. Através da expansão de Taylor das famílias $X_{\lambda}$ e $Y_{\lambda}$, em uma vizinhança das tangências, temos o surgimento de uma função que dá a estabilidade do ponto "foco fundido" dado pela colisão das tangências externas. Em [KGR] há um resumo dessa análise.

Outro artigo que menciona a fórmula para a estabilidade do ponto "foco fundido"é mais recente, do ano de 2001, por Coll, Gasull e Prohens [CGP]. Nesse artigo os autores apresentam uma fórmula que também trata da estabilidade do ponto de colisão entre as duas tangências. Tal fórmula foi obtida através de métodos distintos aos apresentados em $[\mathrm{G}]$ e $[\mathrm{F}]$, não mostraremos como se calcula essa fórmula, que depende da teoria do Blow-up generalizado, apenas daremos os passos iniciais para então enunciá-la. Em [CGP], o sentido das órbitas das famílias $X_{\lambda}$ e $Y_{\lambda}$ são inversos ao do caso $\mathcal{T}_{1}$ de [KGR]. Assim, quando o parâmetro se anula, ocorre a colisão das tangências na origem e o ponto "foco fundido"tem a seguinte forma apresentada na figura 3. Note que na figura 3 a subfigura da esquerda é quando o ponto de colisão entre as tangências é um atrator, ao passo que na subfigura da 
direita o ponto é repulsor.
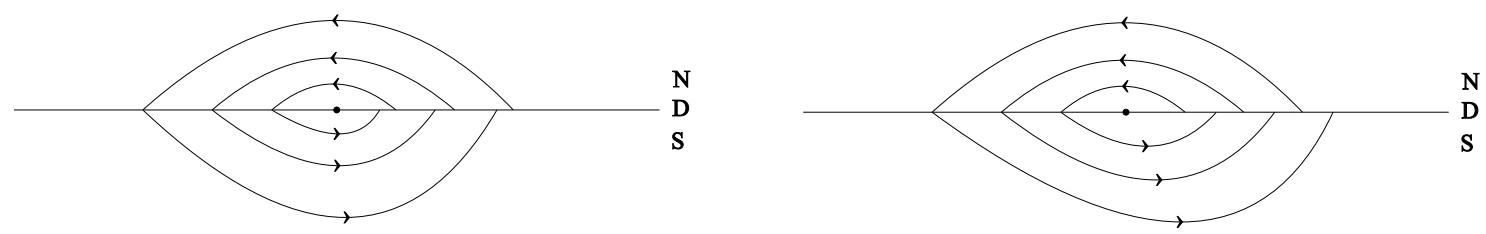

figura 3: ponto de tangência dupla

Para apresentarmos a fórmula do número $V$, definida em [CGP], que dá a existência de ciclo limite e caracteriza a estabilidade do ponto de tangência dupla de um campo vetorial descontínuo $Z=(X, Y)$, vamos supor que os campos $X$ e $Y$ têm a seguinte expansão em séries no ponto $(0,0)$

$$
\begin{gathered}
X(x, y)=\left(a^{+}+b^{+} x+c^{+} y+d^{+} x^{2}+e^{+} x y+f^{+} y^{2}+g^{+} x^{3}+\cdots,\right. \\
\left.l^{+} x+m^{+} y+n^{+} x^{2}+o^{+} x y+p^{+} y^{2}+q^{+} x^{3}+\cdots\right) \\
Y(x, y)=\left(a^{-}+b^{-} x+c^{-} y+d^{-} x^{2}+e^{-} x y+f^{-} y^{2}+g^{-} x^{3}+\cdots,\right. \\
\left.l^{-} x+m^{-} y+n^{-} x^{2}+o^{-} x y+p^{-} y^{2}+q^{-} x^{3}+\cdots\right) .
\end{gathered}
$$

Quando $a^{+}<0, a^{-}>0, l^{+}>0$ e $l^{-}>0$ temos que o campo $Z=(X, Y)$ tem um ponto de tangência dupla na origem. A fórmula que dá a estabilidade desse ponto é dada por

$$
V=\mu^{+}-\mu^{-}
$$

onde

$$
\mu^{ \pm}=\frac{2}{3} \frac{a^{ \pm} n^{ \pm}-\left(b^{ \pm}+m^{ \pm}\right) l^{ \pm}}{a^{ \pm} l^{ \pm}} .
$$

Assim, se $V \neq 0$ então há o aparecimento de um ciclo limite para $Z_{\lambda}$, se $V>0$ então o ciclo limite é repulsor, e se $V<0$ o ciclo limite é atrator.

O que nos propomos a fazer nesse capítulo é calcular o sinal do primeiro número de Lyapunov da regularização de uma família descontínua que tenha $V<0$. Nossa espectativa é que o primeiro número de Lyapunov também seja negativo, uma vez que a família descontínua apresenta um ciclo limite atrator, para valores positivos do parâmetro.

Para manter as convenções definidas em [CGP] com relação ao sentido das órbitas das famílias $X_{\lambda}$ e $Y_{\lambda}$, e assim não precisarmos modificar a expressão que dá o valor de $V$, precisamos modificar a definição do caso $\mathcal{T}_{1}$ para então obter o caso $\mathcal{T}$ que iremos analisar. 
Porém, tal modificação não irá alterar os resultados para o caso $\mathcal{T}_{1}$ pois o que faremos será uma reflexão em torno do eixo do parâmetro, ou seja, faremos um movimento rígido donde concluímos que os resultados serão preservados. Adiante iremos apresentar a expressão da família descontínua que iremos analisar, e é fácil verificar que a forma normal apresentada em $[\mathrm{KGR}]$ para este caso é um caso particular.

Definição 19. Seja $Z_{\lambda}=\left(X_{\lambda}, Y_{\lambda}\right)$ uma FCD a um parâmetro definida em $M$. Dizemos que $Z_{\lambda}$ pertence ao caso $\mathcal{T}$ se para valores negativos e pequenos do parâmetro existirem duas tangências quadráticas externas distintas, uma correspondente ao campo $X_{\lambda}$ com o conjunto de descontinuidade que denotaremos por $p$ e outra do campo $Y_{\lambda}$ com $D$ que denotaremos por $q$. O ponto $p$ deve ser estritamente maior que $q$, e entre esses dois pontos existe um nó atrator de Filippov, que denotaremos por $n_{1}$. Ocorre a colisão de $p$ com $q$ na origem quando $\lambda$ se anula, e para valores positivos e pequenos do parâmetro existe uma órbita periódica atratora tendo no seu interior as duas tangências quadráticas externas, $p$ e $q$, tais que $q$ é maior que $p$. Além disso, existe um nó repulsor de Filippov, denotado por $n_{2}$, entre esses pontos de tangência.

A próxima figura apresenta o diagrama de bifurcação de uma família descontínua que satisfaça os quesitos da definição acima. 


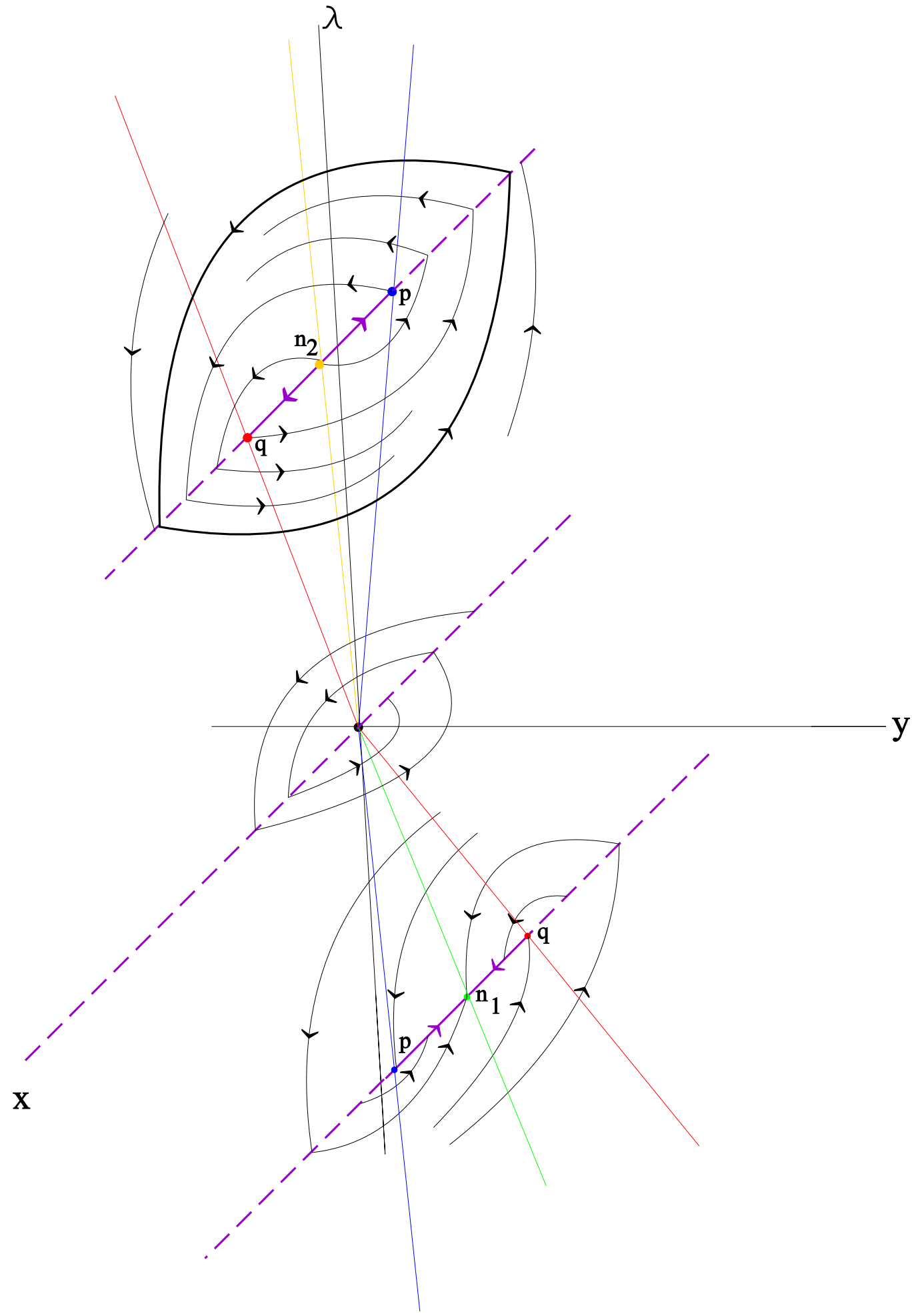

Conjunto de deslizamento

- - - Conjunto de costura

- Ponto de tangência de $\mathrm{X} \lambda$

Ponto de tangência de $\mathrm{Y} \lambda$

Foco atrator

Foco repulsor

figura 4: diagrama de bifurcação do caso $\mathcal{T}$ 
Vamos supor que a tangência quadrática da família $X_{\lambda}$ não mude de posição, ou seja, o ponto $p=(0,0)$ é uma tangência quadrática externa de $X_{\lambda}$ para quaisquer valores de $\lambda$. Como $X_{\lambda}$ não tem singularidades em uma vizinhança de $p$, pelo Teorema do Fluxo Tubular temos que $X_{\lambda}$ é $C^{r}$-conjugado ao campo $(1,0)$. Através de uma rotação de ângulo $\pi$ temos que $X_{\lambda}$ é $C^{r}$-conjugado ao campo $(-1,0)$. Além disso, o difeomorfismo que conjuga esses dois campos preserva o conjunto de descontinuidade. Por outro lado, como o campo $(-1, x)$ não possui nenhuma singularidade, temos que tal campo é $C^{r}$-conjugado a $(1,0)$ que é $C^{r}$ conjugado a $(-1,0)$. Portanto, se $X_{\lambda}$ é uma família de campos cuja órbitas estão descritas na figura $5 \operatorname{logo}$ abaixo, então para cada $\lambda$ o campo $X_{\lambda}$ é $C^{r}$-conjugado ao campo $(-1, x)$. Assim, vamos considerar que

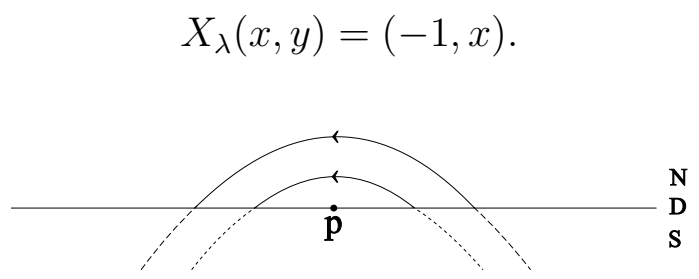

figura 5: $X_{\lambda}$

É fácil verificar que o ponto $p=(0,0)$ é um ponto de tangência quadrática externa de $X_{\lambda}$. Pois, se $X_{\lambda}(x, y)=\left(a_{\lambda}(x, y), b_{\lambda}(x, y)\right)=(-1, x)$ então $X_{\lambda}(F(p))=b_{\lambda}(p)=0$ e

$$
X_{\lambda}^{2}(F(p))=a_{\lambda}(p) \frac{\partial b_{\lambda}}{\partial x}(p)+b_{\lambda}(p) \frac{\partial b_{\lambda}}{\partial y}(p)=-1<0 .
$$

Uma vez que o ponto de tangência quadrática de $X_{\lambda}$ não se desloca, temos que introduzir esse deslocamento do ponto de tangência na família $Y_{\lambda}$. A família $Y_{\lambda}$ que utilizaremos será um pouco mais geral que a família (7.1) e é dada por

$$
\begin{gathered}
Y_{\lambda}(x, y)=\left(a(\lambda)+m(\lambda) x-c(\lambda) y-d(\lambda) x^{2}-e(\lambda) x y-f(\lambda) y^{2}-R(x, y, \lambda),\right. \\
\left.\lambda+l(\lambda) x-m(\lambda) y-n(\lambda) x^{2}-p(\lambda) y^{2}-q(\lambda) x y-S(x, y, \lambda)\right)
\end{gathered}
$$

onde as funções definidas na variável $\lambda$ são suaves, $R(x, y, \lambda)$ e $S(x, y, \lambda)$ são os restos dados por

$$
\begin{aligned}
& R(x, y, \lambda)=a_{21}(\lambda) x^{2} y+a_{12}(\lambda) x y^{2}+a_{30}(\lambda) x^{3}+a_{03}(\lambda) y^{3}+\cdots \\
& S(x, y, \lambda)=b_{21}(\lambda) x^{2} y+b_{12}(\lambda) x y^{2}+b_{30}(\lambda) x^{3}+b_{03}(\lambda) y^{3}+\cdots
\end{aligned}
$$

Note que a diferença entre as expressões da família $Y_{\lambda}$ dado por (7.5) e do campo (7.1) 
se dá no fato que estamos supondo que no primeiro temos o parâmetro $\lambda$ e que, comparando com os coeficientes de (7.1) com os de (7.5) temos que os coeficientes deste último estão todos em função de $\lambda$ e que $b(\lambda)=-m(\lambda)$. Conforme [CGP] para que $Y_{\lambda}$ tenha um ponto de tangência quadrática devemos supor que $l>0$ e $a>0$.

A ideia deste capítulo é, em suma, verificar que o método da regularização do caso tangência dupla quadrática com número $V$ negativo leve à existência de um ponto de Hopf com número de Lyapunov negativo, para valores pequenos do parâmetro de regularização. Por se tratar de uma primeira abordagem a esse problema apresentamos resultados com a inclusão de algumas hipóteses adicionais ao problema. Em um tratamento futuro podemos esperar o enfraquecimento das hipóteses para obtermos os mesmos resultados aqui apresentados, porém com contas maiores que as apresentadas.

Para provar o principal resultado deste capítulo precisaremos de alguns lemas preliminares.

Lema 35. Seja $Z_{\lambda}=\left(X_{\lambda}, Y_{\lambda}\right)$ onde $X_{\lambda}$ e $Y_{\lambda}$ são dados por (7.4) e (7.5), respectivamente, onde $l(0)>0$ e a $(\lambda)$ é uma função suave positiva que satisfaça

H.1) $\frac{d a}{d \lambda}(0) \neq \frac{m(0)}{l(0)+1}$.

Sejam $\varepsilon>0$ e $\varphi$ a função de transição, então existe uma superfície

$$
S=\{(x, \lambda): x=x(y, \varepsilon), \lambda=\lambda(y, \varepsilon)\}
$$

contida no espaço $(x, y, \lambda, \varepsilon) \subset \mathbb{R}^{4}$, cujos pontos são singularidades da família regularizada $Z_{\lambda, \varepsilon}$.

Demonstração. Seja a família de campos vetoriais descontínuos dada por $Z_{\lambda}=\left(X_{\lambda}, Y_{\lambda}\right)$ onde $X_{\lambda}$ e $Y_{\lambda}$ são dados por (7.4) e (7.5), respectivamente. A regularização dessa família, pela 


$$
\begin{aligned}
\text { função } \varphi(y)= & \frac{1}{2}+\frac{y}{2 \sqrt{y^{2}+\varepsilon^{2}}} \text { para } \varepsilon>0, \text { é dada por } \\
Z_{\lambda, \varepsilon}(x, y)= & \left(( \frac { 1 } { 2 } - \frac { y } { 2 \sqrt { y ^ { 2 } + \varepsilon ^ { 2 } } } ) \left(a(\lambda)+m(\lambda) x-c(\lambda) y-d(\lambda) x^{2}-e(\lambda) x y-f(\lambda) y^{2}\right.\right. \\
& -R(x, y, \lambda))-\frac{1}{2}-\frac{y}{2 \sqrt{y^{2}+\varepsilon^{2}}}, \\
& \left(\frac{1}{2}-\frac{y}{2 \sqrt{y^{2}+\varepsilon^{2}}}\right)\left(\lambda+l(\lambda) x-m(\lambda) y-n(\lambda) x^{2}-q(\lambda) x y-p(\lambda) y^{2}\right. \\
& \left.-S(x, y, \lambda))+\left(\frac{1}{2}+\frac{y}{2 \sqrt{y^{2}+\varepsilon^{2}}}\right) x\right)
\end{aligned}
$$

Vamos utilizar o Teorema da Função Implícita para garantirmos a existência da solução das duas componentes da família regularizada. Para isto, efetuamos a seguinte mudança de variável $y=k \varepsilon$, onde $k \in \mathbb{R}$, em $Z_{\lambda, \varepsilon}$. Como $\varepsilon>0$ obtemos então

$$
\begin{aligned}
Z_{\lambda, \varepsilon}(x, k \varepsilon)= & \left(( \frac { 1 } { 2 } - \frac { k } { 2 \sqrt { k ^ { 2 } + 1 } } ) \left(a(\lambda)+m(\lambda) x-c(\lambda) k \varepsilon-d(\lambda) x^{2}-e(\lambda) k \varepsilon x-f(\lambda) k^{2} \varepsilon^{2}\right.\right. \\
& -R(x, k \varepsilon, \lambda))-\frac{1}{2}-\frac{k}{2 \sqrt{k^{2}+1}}, \\
& \left(\frac{1}{2}-\frac{k}{2 \sqrt{k^{2}+1}}\right)\left(\lambda+l(\lambda) x-m(\lambda) k \varepsilon-n(\lambda) x^{2}-q(\lambda) k \varepsilon x-p(\lambda) k^{2} \varepsilon^{2}\right. \\
& \left.-S(x, k \varepsilon, \lambda))+\left(\frac{1}{2}+\frac{k}{2 \sqrt{k^{2}+1}}\right) x\right) .
\end{aligned}
$$

Agora, o determinante Jacobiano de (7.8) calculado em função de $x$ e $\lambda$ quando $x=0$, $\lambda=0 k=0$ e $\varepsilon=0$ vale

$$
\operatorname{det}\left(Z_{\lambda, \varepsilon}\right)(0,0,0)=\frac{1}{4} m(0)-\frac{1}{4} \frac{d a}{d \lambda}(0)(l(0)+1)
$$

que é diferente de zero por hipótese. Assim, pelo Teorema da Função Implícita existe uma vizinhança do ponto $(x, \lambda, k \varepsilon, \varepsilon)=(0,0,0,0)$ onde escrevemos $x$ e $\lambda$ em função de $k \varepsilon=y$ e $\varepsilon$. Ou seja, existe uma superfície $S$ de codimensão 2 no espaço $(x, y, \lambda, \varepsilon) \subset \mathbb{R}^{3} \times \mathbb{R}^{+}$que contém as singularidades da família regularizada.

Lema 36. Seja $Z_{\lambda}=\left(X_{\lambda}, Y_{\lambda}\right)$ onde $X_{\lambda}$ e $Y_{\lambda}$ são dados por (7.4) e (7.5), respectivamente, 
onde $l(0)>0 e$

H.2) $\frac{e(0)}{2}+p(0) \neq 0$.

Sejam $\varepsilon>0$ e $\varphi$ a função de transição, então existe uma curva

$$
T=\{y \in \mathbb{R}: y=y(\varepsilon)\},
$$

contida na superfície $S$ do lema anterior, onde o traço da matriz Jacobiana da família regularizada $Z_{\lambda, \varepsilon}$ se anula.

Demonstração. O traço da matriz Jacobiana da família regularizada é dado por

$$
\begin{aligned}
& \operatorname{tr}\left(D Z_{\lambda, \varepsilon}\right)(x, y, \lambda, \varepsilon)=\left(\frac{1}{2}-\frac{y}{2 \sqrt{y^{2}+\varepsilon^{2}}}\right)(-2 d(\lambda) x-e(\lambda) y-o(\lambda) x-2 p(\lambda) y \\
& \left.\quad-\frac{\partial R}{\partial x}(x, y, \lambda)-\frac{\partial S}{\partial y}(x, y, \lambda)\right)+\varepsilon^{2}\left[x-\lambda-l(\lambda) x+m(\lambda) y+n(\lambda) x^{2}+q(\lambda) x y+p(\lambda) y^{2}\right. \\
& \quad+S(x, y, \lambda)]\left[2\left(y^{2}+\varepsilon^{2}\right)^{3 / 2}\right]^{-1} .
\end{aligned}
$$

Como estamos interessados em singularidades de $Z_{\lambda, \varepsilon}$ onde o traço se anula, da segunda componente de (7.7) obtemos que

$$
-\lambda-l(\lambda) x+m(\lambda) y+n(\lambda) x^{2}+q(\lambda) x y+p(\lambda) y^{2}+S(x, y, \lambda)=-\frac{\left(\sqrt{y^{2}+\varepsilon^{2}}+y\right) x}{\sqrt{y^{2}+\varepsilon^{2}}-y} .
$$

Substituindo esse valor em (7.10) obtemos o traço dado por

$$
\begin{aligned}
& \operatorname{tr}\left(D Z_{\lambda, \varepsilon}\right)(x, y, \lambda, \varepsilon)=\left(\frac{1}{2}-\frac{y}{2 \sqrt{y^{2}+\varepsilon^{2}}}\right)(-2 d(\lambda) x-e(\lambda) y-q(\lambda) x-2 p(\lambda) y \\
& \left.\quad-\frac{\partial R}{\partial x}(x, y, \lambda)-\frac{\partial S}{\partial y}(x, y, \lambda)\right)+\frac{\varepsilon^{2} x}{\left(y^{2}+\varepsilon^{2}\right)\left(\sqrt{y^{2}+\varepsilon^{2}}-y\right)} .
\end{aligned}
$$

Agora, derivando (7.11) em função de $y$ e substituindo $(x, y, \lambda, \varepsilon)$ por $(0,0,0,0)$ obtemos que

$$
\frac{\partial \operatorname{tr}\left(D Z_{\lambda, \varepsilon}\right)}{\partial y}(0,0,0,0)=-\frac{e(0)}{2}-p(0)
$$

que é diferente de zero pela hipótese inicial. Assim, basta aplicar o Teorema da Função Implícita para obtermos o resultado. 
Obs.: Vamos denotar por $p_{H}$ os pontos que são singularidades de $Z_{\lambda, \varepsilon}$ onde o traço da matriz Jacobiana se anula, esses são os candidatos a pontos de Hopf. Note que esses pontos estão todos contidos na curva $T$ do lema anterior.

Os lemas anteriores nos dão elementos essenciais para o cálculo do primeiro número de Lyapunov, pois através desses lemas podemos identificar a real aproximação à origem dos pontos de Hopf $p_{H}=\left(x_{H}, y_{H}, \lambda_{H}, \varepsilon\right)$, todos em função de $\varepsilon$, quando $\varepsilon \rightarrow 0$. É fácil notar que $p_{H}$ depende apenas de $\varepsilon$ pois, pelo Lema 35 temos que $x=x(y, \varepsilon)$ e $\lambda=\lambda(y, \varepsilon)$, ao passo que o Lema 36 afirma que $y=y(\varepsilon)$, portanto o ponto $p_{H}$ é tal que $x_{H}=x(\varepsilon), \lambda_{H}=\lambda(\varepsilon) \mathrm{e}$ $y_{H}=y(\varepsilon)$.

Proposição 37. Seja $Z_{\lambda}=\left(X_{\lambda}, Y_{\lambda}\right)$ onde $X_{\lambda}$ e $Y_{\lambda}$ são dados por (7.4) e (7.5), respectivamente. Vamos supor que ${ }^{1}>0$, para todo $\lambda$, que valem as hipóteses H.1) e H.2) dos lemas anteriores e também as seguintes hipóteses

H.3) $\beta=\frac{q}{2}+d \neq 0$

H.4) $(2 m \beta-e)^{2}+(a-1)\left(8 \beta d c+2 d c^{2}+2 m^{2} f+2 e m c+4 d f(1-a)+a e(e-1) \geq 0\right.$

H.5) $\alpha_{ \pm}=m(2+c)+e(a-1) \pm \zeta \neq 0$ onde

$$
\zeta=\left((2 m \beta-e)^{2}+(a-1)\left(8 \beta d c+2 d c^{2}+2 m^{2} f+2 e m c+4 d f(1-a)+a e(e-1)\right)^{1 / 2} .\right.
$$

H.6) $\gamma=\beta m-\alpha_{ \pm}(1+l) \neq 0$.

Sejam $\varepsilon>0$ e $\varphi$ a função de transição. Os pontos $p_{H}=\left(x_{H}, y_{H}, \lambda_{H}, \varepsilon\right) \in T$, onde $T$ é a curva do Lema 36, se aproximam de $(0,0,0,0)$ quadraticamente quando $\varepsilon$ vai a zero, ou seja, podemos escrever esse ponto em função de $\varepsilon$ como

$$
\begin{aligned}
& x_{H}=\alpha \varepsilon^{2}, \\
& y_{H}=\beta \varepsilon^{2}, \\
& \lambda_{H}=\gamma \varepsilon^{2} .
\end{aligned}
$$

Demonstração. Para verificar essa proposição vamos iniciar com a equação

$$
\operatorname{tr}\left(D Z_{\lambda, \varepsilon}\right)(x, y, \lambda, \varepsilon)=0
$$

\footnotetext{
${ }^{1}$ Para simplificar a notação não vamos escrever a dependência dos coeficientes da família vetorial descontínua em relação a $\lambda$, portanto, por exemplo, ao invés de escrevermos $m(\lambda(\varepsilon))$ vamos escrever simplemente $m$.
} 
onde o lado esquerdo dessa equação é dado por (7.11). Nosso objetivo é calcular o menor coeficiente não nulo da expansão de $y_{H}$, próximo da origem, dada por

$$
y_{H}=\beta_{0}+\beta_{1} \varepsilon+\beta_{2} \varepsilon^{2}+\beta_{3} \varepsilon^{3}+\cdots
$$

Porém, note que o coeficiente $\beta_{0}$ é nulo, isto se deve ao fato que quando $\varepsilon$ vai a zero devemos ter que $y_{H}=0$, implicando que $\beta_{0}=0$.

Manipulando algebricamente a equação (7.12) podemos reduzi-la a

$$
-8 \Gamma x y^{3}-\Gamma^{2} \varepsilon^{2} y^{2}-8 \Gamma \varepsilon^{2} x y+4 \varepsilon^{2} x^{2}-\Gamma^{2} \varepsilon^{4}=0
$$

onde

$$
\Gamma=-2 d(\lambda) x-e(\lambda) y-q(\lambda) x-2 p(\lambda) y-\frac{\partial R}{\partial x}(x, y, \lambda)-\frac{\partial S}{\partial y}(x, y, \lambda) .
$$

Para verificar qual o primeiro coeficiente da expansão de $y_{H}$ que não se anula, vamos substituir

$$
\begin{aligned}
& x=\alpha \varepsilon, \\
& \lambda=\gamma \varepsilon \\
& y=\beta_{1} \varepsilon+\beta_{2} \varepsilon^{2}+\beta_{3} \varepsilon^{3}
\end{aligned}
$$

em (7.13). Teremos então um polinômio em $\varepsilon$, e igualando a zero cada coeficiente desse polinômio teremos então os valores de $\beta_{i}, i=1,2,3$. Começamos com a expansão até a ordem 3 pois caso encontremos um coeficiente não nulo não precisamos seguir adiante colocando os outros termos da expansão de $y_{H}$.

As derivadas dos restos são dadas por

$$
\begin{aligned}
\frac{\partial R}{\partial x} & (x, y, \lambda)=a_{12} y^{2}+a_{13} y^{3}+a_{14} y^{4}+\cdots+a_{1 n} y^{n}+\cdots \\
& +2\left(a_{21} y+a_{22} y^{2}+a_{23} y^{3}+a_{24} y^{4}+\cdots+a_{2 n} y^{n}+\cdots\right) x \\
& +3\left(a_{30}+a_{31} y+a_{32} y^{2}+a_{33} y^{3}+a_{34} y^{4}+\cdots+a_{3 n} y^{n}+\cdots\right) x^{2} \\
& +4\left(a_{40}+a_{41} y+a_{42} y^{2}+a_{43} y^{3}+a_{44} y^{4}+\cdots+a_{4 n} y^{n}+\cdots\right) x^{2} \\
& +\cdots
\end{aligned}
$$




$$
\begin{aligned}
& \frac{\partial S}{\partial x}(x, y, \lambda)=b_{21} x^{2}+b_{31} x^{3}+b_{41} x^{4}+\cdots+b_{n 1} x^{n}+\cdots \\
& \quad+2\left(b_{12} x+b_{22} x^{2}+b_{32} x^{3}+b_{42} x^{4}+\cdots+b_{n 2} x^{n}+\cdots\right) y \\
& \quad+3\left(b_{03}+b_{13} x+b_{23} x^{2}+b_{33} x^{3}+b_{43} x^{4}+\cdots+b_{n 3} x^{n}+\cdots\right) y^{2} \\
& \quad+4\left(b_{04}+b_{14} x+b_{24} x^{2}+b_{34} x^{3}+b_{44} x^{4}+\cdots+b_{n 4} x^{n}+\cdots\right) y^{2} \\
& \quad+\cdots
\end{aligned}
$$

Não utilizaremos todos os termos dessas derivadas pois as contas ficariam muito grandes, como estamos interessados apenas nos coeficientes de ordens baixas, vamos considerar que as derivadas acima se restringem a

$$
\begin{aligned}
\frac{\partial R}{\partial x}(x, y, \lambda)= & a_{12} y^{2}+a_{13} y^{3}+2\left(a_{21} y+a_{22} y^{2}\right) x+3\left(a_{30}+a_{31} y+a_{32} y^{2}\right) x^{2}+4\left(a_{40}\right. \\
& \left.+a_{41} y\right) x^{3} \\
\frac{\partial S}{\partial x}(x, y, \lambda)= & b_{21} x^{2}+b_{31} x^{3}+2\left(b_{12} x+b_{22} x^{2}\right) y+3\left(b_{03}+b_{13} x+b_{23} x^{2}\right) y^{2}+4\left(b_{04}\right. \\
& \left.+b_{14} x\right) y^{4} .
\end{aligned}
$$

Substituindo (7.14) em (7.15) e (7.16), e depois substituindo esses valores na equação (7.13) obtemos então uma equação polinomial. Não apresentaremos essa equação por se tratar de um polinômio muito grande. Igualando os coeficientes desse polinômio a zero, obtemos então que $\beta_{1}=0, \beta_{2}=\frac{q(\lambda(\varepsilon))}{8}+\frac{d(\lambda(\varepsilon))}{4}$ e $\beta_{3}=0$.

Agora, vamos trabalhar com a primeira componente de $Z_{\lambda, \varepsilon}$ para verificar como a curva ordenada das singularidades tende a zero com $\varepsilon$. Manipulando a equação

$$
\begin{aligned}
& \left(\frac{1}{2}-\frac{y}{2 \sqrt{y^{2}+\varepsilon^{2}}}\right)\left(a(\lambda)+m(\lambda) x-c(\lambda) y-d(\lambda) x^{2}-e(\lambda) x y-f(\lambda) y^{2}-R(x, y, \lambda)\right) \\
& \quad-\frac{1}{2}-\frac{y}{2 \sqrt{y^{2}+\varepsilon^{2}}}=0
\end{aligned}
$$

obtemos então

$$
\varepsilon^{2} \Upsilon^{2}-2\left(2 y^{2}+\varepsilon^{2}\right) \Upsilon+\varepsilon^{2}=0,
$$


onde

$$
\Upsilon=a(\lambda)+m(\lambda) x-c(\lambda) y-d(\lambda) x^{2}-e(\lambda) x y-f(\lambda) y^{2}-R(x, y, \lambda) .
$$

Vamos utilizar o resto $R(x, y, \lambda)$ até a ordem 4.

Substituindo os valores $y=\beta \varepsilon^{2}, x=\alpha_{1} \varepsilon+\alpha_{2} \varepsilon^{2}+\alpha_{3} \varepsilon^{3}$ e $\lambda=\gamma \varepsilon$ em (7.17) obtemos um polinômio em $\varepsilon$, e igualando seus coeficientes a zero obtemos que $\alpha_{1}=\alpha_{3}=0$ e

$$
\alpha_{2}=-[2 m-e+a e+m c \pm \zeta] \beta\left[-2 d+2 a d-m^{2}\right]^{-1}
$$

onde

$$
\zeta=\left((2 m \beta-e)^{2}+(a-1)\left(8 \beta d c+2 d c^{2}+2 m^{2} f+2 e m c+4 d f(1-a)+a e(e-1)\right)^{1 / 2} .\right.
$$

Para que esse valor de $\alpha_{2}$ faça sentido devemos primeiramente supor que o termo dentro da raiz de $\zeta$ seja positivo. Assim, supondo que $2 m-e+a e+m c \pm \zeta \neq 0$ temos o resultado para $x$.

Para finalizar vamos calcular $\lambda$ através da segunda componente de $Z_{\lambda, \varepsilon}$. Manipulando a equação

$$
\begin{aligned}
\left(\frac{1}{2}\right. & \left.-\frac{y}{2 \sqrt{y^{2}+\varepsilon^{2}}}\right)\left(\lambda+l(\lambda) x-m(\lambda) y-n(\lambda) x^{2}-q(\lambda) x y-p(\lambda) y^{2}-S(x, y, \lambda)\right) \\
& +\left(\frac{1}{2}+\frac{y}{2 \sqrt{y^{2}+\varepsilon^{2}}}\right) x=0
\end{aligned}
$$

obtemos

$$
4 y^{2} \Theta x+\varepsilon^{2} \Theta^{2}+2 \varepsilon^{2} \Theta x+\varepsilon^{2} x^{2}=0
$$

onde

$$
\Theta=\lambda+l(\lambda) x-m(\lambda) y-n(\lambda) x^{2}-q(\lambda) x y-p(\lambda) y^{2}-S(x, y, \lambda) .
$$

Neste caso tambem iremos considerar o resto com termos até a ordem 4. Assim, substituindo $x=\alpha \varepsilon^{2}, y=\beta \varepsilon^{2}$ e $\lambda=\gamma_{1} \varepsilon+\gamma_{2} \varepsilon^{2}+\gamma_{3} \varepsilon^{3}$ em (7.19) obtemos que $\gamma_{1}=0, \gamma_{3}=0$ e $\gamma_{2}=\beta m-\alpha(1+l)$ que não se anula por hipótese.

Antes de prosseguirmos vamos verificar que o determinante Jacobiano aplicado em $p_{H}$ é positivo. Calculando o determinante Jacobiano em $x=\alpha \varepsilon^{2}, y=\beta \varepsilon^{2}$ e $\lambda=\gamma \varepsilon^{2}$ e notando que os restos $R$ e $S$, bem como suas derivadas, se anulam quando $\varepsilon$ vai a zero obtemos o 
seguinte limite

$$
\lim _{\varepsilon \rightarrow 0^{+}} \operatorname{det}\left(Z_{\lambda, \varepsilon}\right)\left(x_{H}, y_{H}, \lambda_{H}, \varepsilon\right)=\operatorname{sinal}[(a(0)+1)(l(0)+1)] \infty=+\infty
$$

uma vez que tanto $l(0)$ quanto $a(0)$ são positivos. Assim, pelo limite acima, temos que o determinante Jacobiano em $p_{H}$ é positivo, uma vez que estamos interessados em valores de $\varepsilon$ muito próximos de zero.

Agora vamos enunciar e demonstrar o principal resultado deste capítulo que trata da conexão entre o número $V$ de uma família descontínua de campos vetoriais e o primeiro número de Lyapunov da regularização dessa família.

Teorema 38. Seja $Z_{\lambda}=\left(X_{\lambda}, Y_{\lambda}\right)$ uma família de campos vetoriais descontínuos dada por

$$
\begin{aligned}
X_{\lambda}(x, y)= & (-1, x) \\
Y_{\lambda}(x, y)= & \left(a(\lambda)+m(\lambda) x-c(\lambda) y-d(\lambda) x^{2}-e(\lambda) x y-f(\lambda) y^{2}-R(x, y, \lambda),\right. \\
& \left.\lambda+l(\lambda) x-m(\lambda) y-n(\lambda) x^{2}-q(\lambda) x y-p(\lambda) y^{2}-S(x, y, \lambda)\right)
\end{aligned}
$$

onde os restos $R$ e $S$ são dados por (7.6). Vamos supor que os coeficientes de $Y_{\lambda}$ satisfaçam as hipóteses H.1) - H.6) e que, além disso, tenhamos

H.7) $a(0)>1$,

H.8) $l(0)<\frac{5(a(0)-1)}{a(0)+1}$

H.9) $m(\lambda)=\eta \varepsilon^{2}$, com $\eta \neq 0$.

Sejam $\varepsilon>0$, e $\varphi(y)=\frac{1}{2}+\frac{y}{2 \sqrt{y^{2}+\varepsilon^{2}}}$. Se $V<0$, onde $V$ é dado por (7.2), então a família regularizada $Z_{\lambda, \varepsilon}$ possui um ponto de Hopf cujo primeiro número de Lyapunov é negativo.

Demonstração. Os resultados anteriores deste capítulo nos levam a um único ponto $p_{H}=$ $\left(\alpha \varepsilon^{2}, \beta \varepsilon^{2}, \gamma \varepsilon^{2}, \varepsilon\right)$, a unicidade vem do Teorema da Função Implícita, que é uma singularidade que tem determinante Jacobiano positivo e o traço da matriz Jacobiana é nulo, para a família regularizada $Z_{\lambda, \varepsilon}$ dada por (7.7).

A expressão do primeiro número de Lyapunov $l_{1}$ está no capítulo 3 , veja a fórmula (3.32). Para calculá-lo precisamos calcular as derivadas até a terceira ordem das componentes de $Z_{\lambda, \varepsilon}$ e depois verificar qual é o limite quando fazemos $\varepsilon$ tender a zero. Para isso, vamos 
verificar qual o menor termo das derivadas dos restos e substitui-los na fórmula de $l_{1}$. Assim, temos que

$$
\begin{aligned}
& \frac{\partial R}{\partial x}\left(p_{H}\right)=r_{1} \varepsilon^{4}, \\
& \frac{\partial R}{\partial y}\left(p_{H}\right)=r_{2} \varepsilon^{4}, \\
& \frac{\partial^{2} R}{\partial x^{2}}\left(p_{H}\right)=r_{3} \varepsilon^{2}, \\
& \frac{\partial^{2} R}{\partial x \partial y}\left(p_{H}\right)=r_{4} \varepsilon^{2}, \\
& \frac{\partial^{2} R}{\partial y^{2}}\left(p_{H}\right)=r_{5} \varepsilon^{2}, \\
& \frac{\partial^{3} R}{\partial x^{3}}\left(p_{H}\right)=r_{6}+r_{7} \varepsilon^{2}, \\
& \frac{\partial^{3} R}{\partial x^{2} \partial y}\left(p_{H}\right)=r_{8}+r_{9} \varepsilon^{2}, \\
& \frac{\partial^{3} R}{\partial y^{2} \partial x}\left(p_{H}\right)=r_{10}+r_{11} \varepsilon^{2}, \\
& \frac{\partial^{3} R}{\partial y^{3}}\left(p_{H}\right)=r_{12}+r_{13} \varepsilon^{2},
\end{aligned}
$$

analogamente para o resto $S$, ou seja,

$$
\begin{aligned}
& \frac{\partial S}{\partial x}\left(p_{H}\right)=s_{1} \varepsilon^{4}, \quad \frac{\partial S}{\partial y}\left(p_{H}\right)=s_{2} \varepsilon^{4}, \quad \frac{\partial^{2} S}{\partial x^{2}}\left(p_{H}\right)=s_{3} \varepsilon^{2}, \\
& \frac{\partial^{2} S}{\partial x \partial y}\left(p_{H}\right)=s_{4} \varepsilon^{2} \\
& \frac{\partial^{2} S}{\partial y^{2}}\left(p_{H}\right)=s_{5} \varepsilon^{2}, \\
& \frac{\partial^{3} S}{\partial x^{3}}\left(p_{H}\right)=s_{6}+s_{7} \varepsilon^{2}, \\
& \frac{\partial^{3} S}{\partial x^{2} \partial y}\left(p_{H}\right)=s_{8}+s_{9} \varepsilon^{2}, \\
& \frac{\partial^{3} S}{\partial y^{2} \partial x}\left(p_{H}\right)=s_{10}+s_{11} \varepsilon^{2}, \\
& \frac{\partial^{3} S}{\partial y^{3}}\left(p_{H}\right)=s_{12}+s_{13} \varepsilon^{2} \text {. }
\end{aligned}
$$

Substituindo esses valores acima e os valores encontrados de $p_{H}$ na fórmula de $l_{1}$, e calculando o limite de $l_{1}$ quando $\varepsilon$ vai a zero obtemos

$$
\begin{aligned}
\lim _{\varepsilon \rightarrow 0^{+}} l_{1} & =-\operatorname{sinal}(-5 n(0) a(0)+n(0) a(0) l(0)+5 n(0)+n(0) l(0)) \infty \\
& =-\operatorname{sinal}\left(\frac{n(0)}{l(0)}(-5 a(0)+a(0) l(0)+5+l(0))\right) \infty
\end{aligned}
$$

Agora, como estamos supondo que o número $V$ de Coll-Gasull-Prohens da família descontínua $Z_{\lambda}$ é negativo, sendo que $V=n(0) / l(0)<0$, e sendo que $a(0)>1$ e $l(0)<5 \frac{a(0)-1}{a(0)+1}$ então o primeiro número de Lyapunov é negativo, o que finaliza a prova do teorema.

A próxima figura apresenta o diagrama de bifurcação do caso regularizado, para um $\varepsilon>0$ pequeno fixado. 

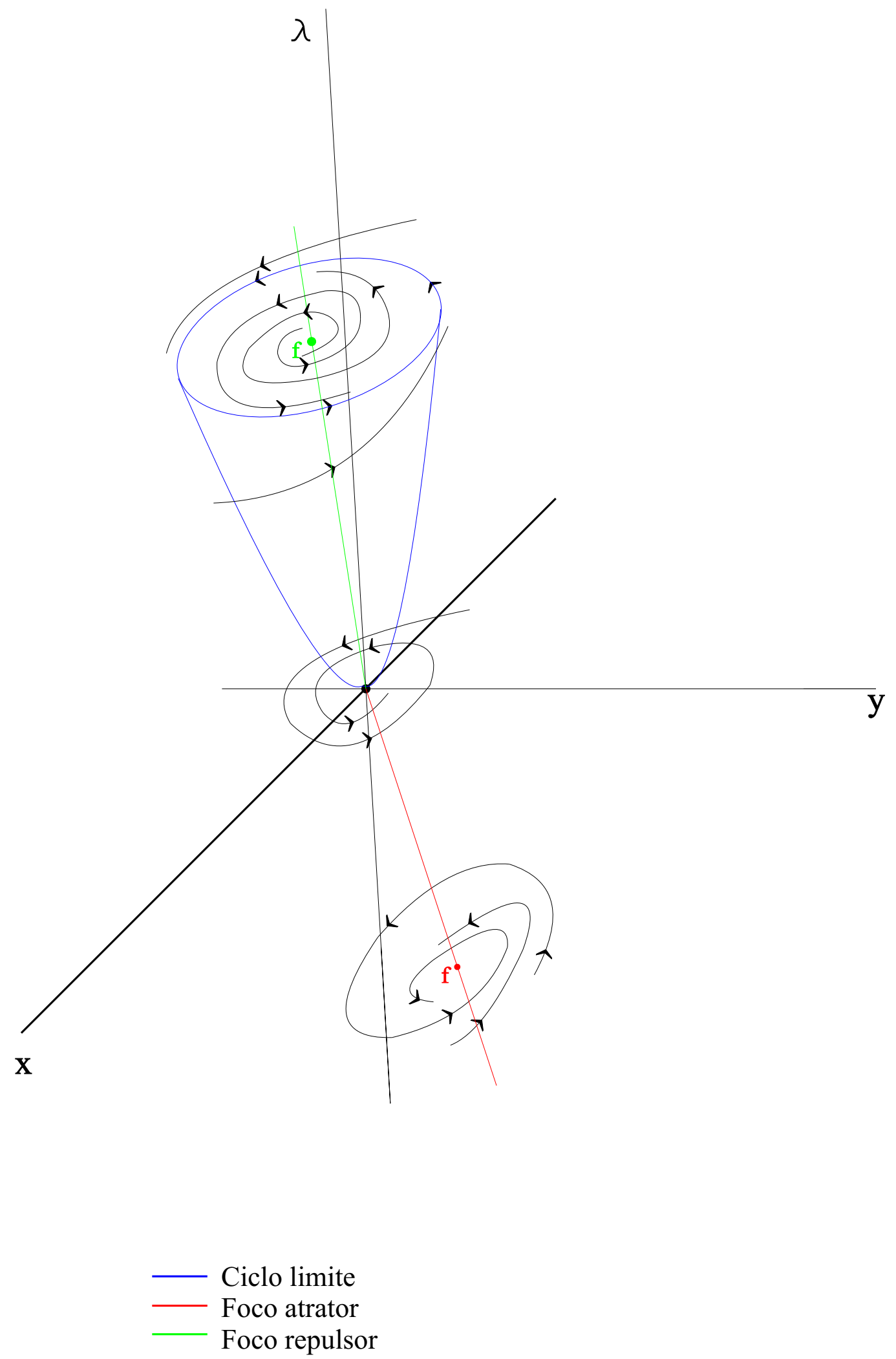

figura 6: diagrama de bifurcação da regularização do caso $\mathcal{T}$ 


\section{Capítulo 8}

\section{Exemplo analisado}

Neste capítulo vamos analisar as bifurcações de um exemplo de campos vetoriais descontínuos via regularização. A primeira seção deste capítulo estima as bifurcações que ocorrem quando uma família a um parâmetro de campos vetoriais descontínuos apresenta uma sela e um nó no campo de Filippov, para valores pequenos e negativos do parâmetro, que colidem quando o parâmetro se anula. Este caso corresponde ao caso 3.3 de [KGR] entitulado colisão de pseudo-equilíbrios, e a bifurcação descrita no artigo é chamada de "Bifurcação pseudo-sela-nó."

O caso da primeira seção será considerado como um exemplo pois foge ao escopo do trabalho no sentido que não é definido usando as propriedades que o caracterizam analiticamente, ou seja, o campo $P(x, \lambda)$ deve satisfazer $\left.P(x, \lambda)\right|_{x=0}=0,\left.P^{\prime}(x, \lambda)\right|_{x=0}=0$,

$\left.P^{\prime \prime}(x, \lambda)\right|_{x=0}=0$ e $\left.\frac{\partial P(x, \lambda)}{\partial \lambda}\right|_{x=0}=0$. Ao invés disto, definimos a família de campos vetoriais descontínuos a ser analisada pela sua forma normal topológica dada em [KGR].

\subsection{Bifurcação sela e nó no bordo via regularização}

A expressão da família a um parâmetro de campos vetoriais descontínuos será dada por $Z_{\lambda}=\left(X_{\lambda}, Y_{\lambda}\right)$ onde

$$
\begin{aligned}
& X_{\lambda}(x, y)=\left(x^{2}+\lambda, a\right) \\
& Y_{\lambda}(x, y)=(b, c)
\end{aligned}
$$

onde $a<0, b>0$ e $c>0$.

A definição do caso sela e nó no bordo é a seguinte:

Definição 20. Seja $Z_{\lambda}=\left(X_{\lambda}, Y_{\lambda}\right)$ uma FCD definida em $M$ onde $Y_{\lambda}=(b, c)$ com $b>0$ e $c>0$. Dizemos que $Z_{\lambda}$ pertence ao caso $\mathcal{S} \mathcal{N}$ se para valores negativos do parâmetro possui 
um nó atrator de Filippov, $n$, e uma sela de Filippov, s. Quando o parâmetro se anula $s$ e $n$ colidem e não existem mais singularidades para valores positivos e pequenos do parâmetro. 


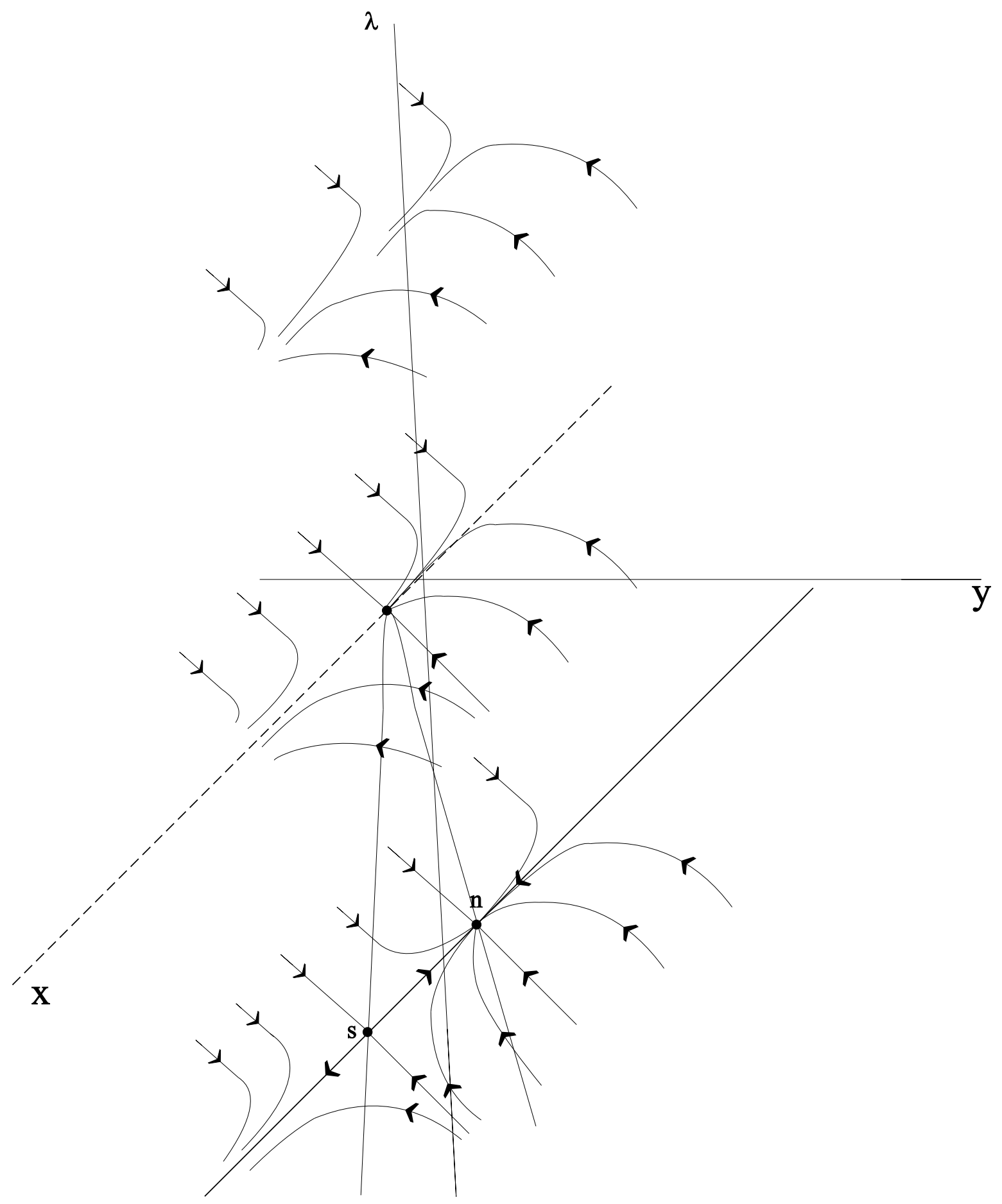

figura 1: diagrama de bifurcação do caso $\mathcal{S N}$. 
De (8.1) notamos que o conjunto de deslizamento é todo o eixo- $x$. De fato, pois

$$
X_{\lambda} F(p) Y_{\lambda} F(p)=a c<0,
$$

para quaisquer valores de $x$ e de $\lambda$. Por outro lado, o campo de filippov, definido em todo o eixo real, é dado por

$$
F_{Z_{\lambda}}(x, 0)=\left(\frac{c\left(x^{2}+\lambda\right)-a b}{c-a}, 0\right) .
$$

Donde concluimos que existem duas singularidades do campo de Filippov, dadas por $x_{ \pm}=$ $\pm \sqrt{\frac{a b}{c}-\lambda}$, para valores do parâmetro menores que $\frac{a b}{c}<0$. Note que no caso apresentado no artigo [KGR] temos que $\frac{a b}{c}=0$, pois os autores assumem que $a=1, b=0$ e $c=-1$. Quando o valor do parâmetro atinge $\frac{a b}{c}$ existe uma única singularidade do campo de Filippov, e para parâmetros maiores que esse valor não existem singularidades do campo de Filippov, isto se deve ao fato do campo de Filippov ter uma expressão quadrática em $x$. Por essas observações temos que a família dada por (8.1) é do tipo $\mathcal{S N}$.

Lema 39. Seja $Z_{\lambda}$ dada por (8.1). Então existe um valor $\lambda^{*}$ do parâmetro tal que a familia regularizada possui uma única singularidade quando o parâmetro vale $\lambda^{*}$, não possui singularidades para valores de $\lambda$ maiores que $\lambda^{*}$ e possui duas singularidades quando $\lambda<\lambda^{*}$.

Demonstração. De (8.1) e usando a função de transição dos capítulos anteriores obtemos a seguinte expressão para a família regularizada

$$
Z_{\lambda, R}(x, y)=\left(\frac{b+x^{2}+\lambda}{2}+\frac{y\left(-b+x^{2}+\lambda\right)}{2 \sqrt{y^{2}+\varepsilon^{2}}}, \frac{a+c}{2}+\frac{y(a-c)}{2 \sqrt{y^{2}+\varepsilon^{2}}}\right)
$$

onde $\varepsilon>0$.

As singularidades desse campo são pontos tais que a ordenada e o parâmetro satisfazem

$$
\begin{aligned}
& y_{s}= \pm \sqrt{-\frac{1}{4 a c}}(a+c) \varepsilon \\
& \lambda_{s}=\frac{b a}{c}-x^{2} .
\end{aligned}
$$

Da expressão de $\lambda_{s}$ notamos que existem valores do parâmetro onde existem duas, uma ou nenhuma singularidade do campo $Z_{\lambda, R}$. É fácil verificar que o valor do parâmetro onde 
$Z_{\lambda, R}$ tem uma única singularidade é dado por

$$
\lambda^{*}=\frac{b a}{c}
$$

ou, equivalentemente, quando $x^{*}=0$. Para valores do parâmetro menores que $\lambda^{*}$ o campo regularizado tem duas singularidades e para valores maiores não existe nenhuma singularidade.

Através de cálculos numéricos notamos que a singularidade que deve ser considerada é a que tem ordenada dada por $y=\sqrt{-\frac{1}{4 a c}}(a+c) \varepsilon$. Portanto, de agora em diante iremos considerar apenas essa ordenada.

Vamos utilizar o Teorema 14, do capítulo "Foco no bordo", para provar o principal resultado deste capítulo dado pelo próximo teorema.

Proposição 40. Seja $Z_{\lambda}$ uma familia a um parâmetro de campos vetoriais descontínuos dada por (8.1). Então, a respectiva família regularizada possui uma bifurcação sela-nó quando o parâmetro vale $\lambda^{*}$.

Demonstração. O traço e o determinante jacobiano de (8.2) são dados por

$$
\begin{aligned}
\operatorname{det} & =\frac{(a-c) x \varepsilon^{2}\left(y+\sqrt{y^{2}+\varepsilon^{2}}\right)}{2\left(y^{2}+\varepsilon^{2}\right)^{2}} \\
\operatorname{tr} & =x+\frac{x y}{\left(y^{2}+\varepsilon^{2}\right)^{1 / 2}}+\frac{(a-c) \varepsilon^{2}}{2\left(y^{2}+\varepsilon^{2}\right)^{3 / 2}} .
\end{aligned}
$$

Substituindo o valor da ordenada nas expressões acima, verificamos que o traço é negativo e $\Delta=(\operatorname{tr})^{2}-4$ det $>0$ o que nos garante a existência de um nó atrator.

Notamos que quando $x=0$ o determinante Jacobiano se anula, e precisamos desse fato para que um dos seus autovalores seja nulo. Portanto, o ponto

$$
\left(x^{*}, y^{*}, \lambda^{*}\right)=\left(0, \sqrt{-\frac{1}{4 a c}}(a+c) \varepsilon, \frac{a b}{c}\right)
$$

é a singularidade onde a matriz do Jacobiano da FCR, no parâmetro $\lambda^{*}$, tem um autovalor $\mu_{1}$ nulo. Para finalizar (SN1) vamos apresentar os autovetores à direita e à esquerda do autovalor nulo. 
Um autovetor à direita é o vetor não-nulo $v=\left(v_{1}, v_{2}\right)$ que satisfaz $A\left(p^{*}, \lambda^{*}\right) v=\mu_{1} v=0$, pois queremos associá-lo ao autovalor $\mu_{1}=0$. Assim,

$$
v=\left(1, \frac{2 x^{*}\left(\left(y^{*}\right)^{2}+\varepsilon^{2}\right)+\left(y^{*}+\sqrt{\left(y^{*}\right)^{2}+\varepsilon^{2}}\right)}{\varepsilon^{2}\left(b-\left(x^{*}\right)^{2}-\lambda^{*}\right)}\right)
$$

e substituindo os valores de $x^{*}, y^{*}$ e $\lambda^{*}$ obtemos que

$$
v=(1,0)
$$

Analogamente, o autovetor à esquerda, associado ao autovalor $\mu_{1}=0$, é a solução nãonula de $w^{T} A\left(p^{*}, \lambda^{*}\right)=0$ que é dado por

$$
w=\left(1, \frac{b-\left(x^{*}\right)^{2}-\lambda^{*}}{a-c}\right)
$$

e substituindo os valores de $x^{*}$ e $\lambda^{*}$ dados por (8.5) obtemos

$$
w=\left(1,-\frac{b}{c}\right)
$$

Isso finaliza a verificação da hipótese (SN1) do Teorema 14, agora vamos às outras hipóteses.

Temos que verificar que o seguinte produto escalar é não-nulo

$$
p_{1}=\left\langle w, \frac{d}{d \lambda} F\left(p^{*}, \lambda^{*}\right)\right\rangle .
$$

Fazendo os cálculos, de acordo com a prova da proposição 15 do capítulo "foco no bordo", obtemos que

$$
p_{1}=\frac{1}{2}+\frac{y}{2 \sqrt{y^{2}+\varepsilon^{2}}}
$$

que obviamente nunca se anula.

Agora, vamos verificar que o seguinte produto escalar é não-nulo

$$
p_{2}=\left\langle w, D_{x}^{2} Z_{\lambda, \varepsilon}\left(p^{*}, \lambda^{*}\right)(v, v)\right\rangle
$$

Fazendo as contas para achar $p_{2}$ e substiuindo os valores do ponto (8.5) obtemos finalmente 
que

$$
p_{2}=1+\frac{y}{\sqrt{y^{2}+\varepsilon^{2}}},
$$

que também não se anula para quaisquer valores de $y$ e $\varepsilon$. Fica provado assim o teorema.

A próxima figura apresenta o diagrama de bifurcação para uma família regularizada referente a uma FCD que seja do tipo $\mathcal{S N}$. 

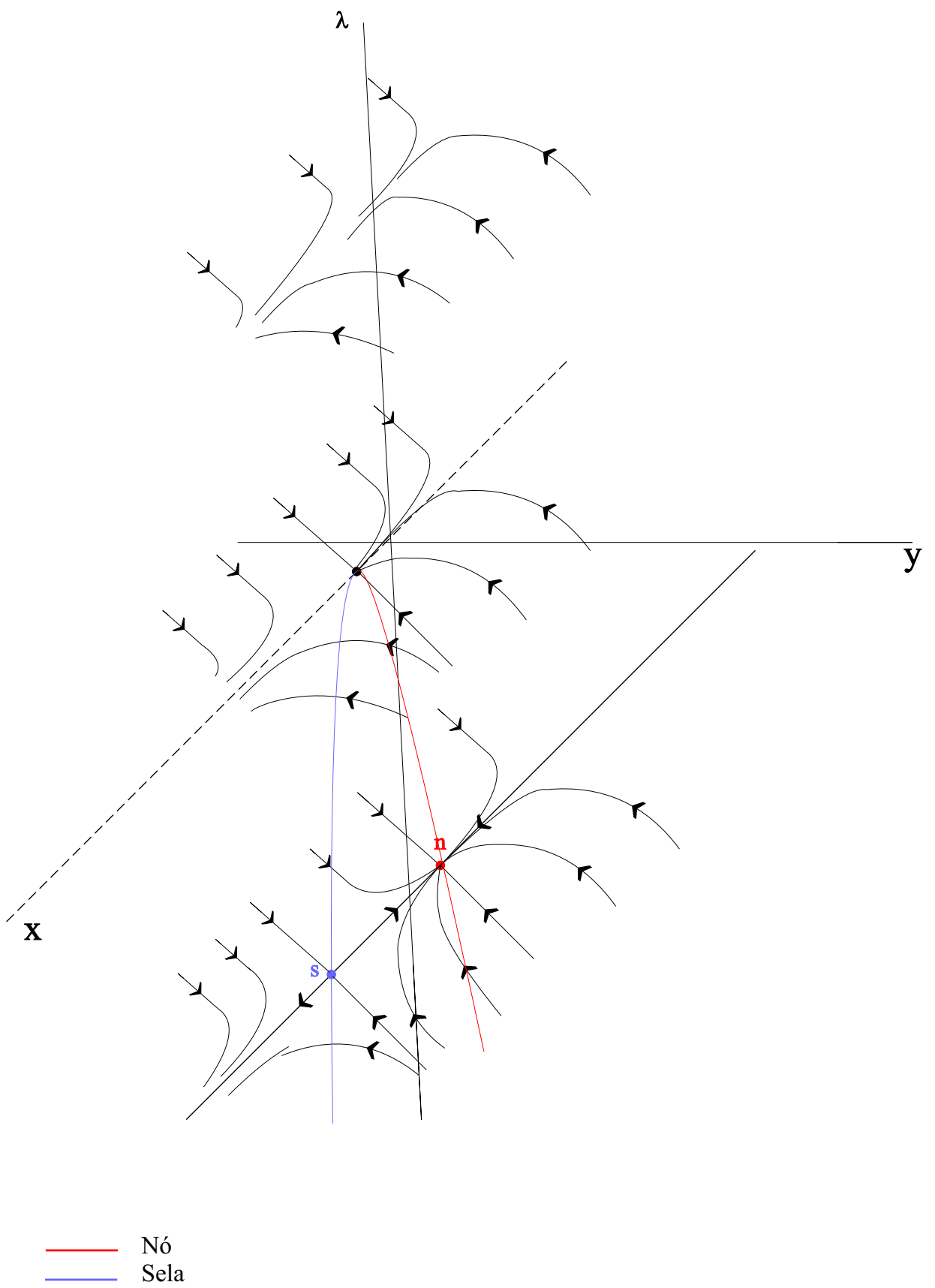

figura 2: diagrama de bifurcação de uma família regularizada do caso $\mathcal{S N}$. 


\section{Capítulo 9}

\section{Conclusões}

No artigo [KGR] os autores apresentam uma lista de bifurcações de codimensão um de campos vetoriais descontínuos. Esta tese teve como objetivo explicar as bifurcações que ocorrem em alguns casos desta lista pela teoria clássica das bifurcações de campos vetoriais suaves, via o método da regularização. Apresentamos abaixo um escopo dos resultados obtidos.

O Teorema 20 do capítulo 3, veja página 94, explica as bifurcações que ocorrem no primeiro caso de foco no bordo. Para valores pequenos do parâmetro temos uma bifurcação de Hopf e logo após uma bifurcação sela-nó. A prova deste teorema está contida ao longo das proposições do capítulo 3 que estão colocadas anteriormente ao enunciado do Teorema 20. Na página 96 está a figura que contém o diagrama de bifurcação da regularização do primeiro caso de foco no bordo. O Teorema 21 na página 97 explica a bifurcação do segundo caso de foco no bordo através da bifurcação do tipo sela-nó que ocorre na respectiva família regularizada. O seu diagrama de bifurcações está apresentado na página 98. O subcaso intermediário a esses dois subcasos citados, é explicado por uma bifurcação de codimensão dois no Teorema 28 da página 116. A explicação para este cubcaso é que há um ponto de Bogdanov-Takens para a família regularizada. Uma explicação do diagrama de bifurcação está contida na seção 3.6. No final deste capítulo, na página 129 temos uma tabela que resume os resultados obtidos pela análise desses 3 primeiros subcasos.

O Teorema 29 da página 131 explica as bifurcações que ocorrem no terceiro caso de foco no bordo, há a presença de uma bifurcação do tipo sela-nó como exemplifica o diagrama da página 134. O Teorema 31 da página 135 explica os dois últimos subcasos de foco no bordo. Em um desses subcasos ocorre uma bifurcação do tipo sela-nó e no outro ocorre apenas uma mudança de foco para nó. Os diagramas de bifurcação desses casos estão contidos nas páginas 137 e 138. Na página 139 há uma tabela que resume os resultados destes casos de 
foco no bordo.

No Teorema 33 da página 146 temos a explicação de que nos dois subcasos de sela no bordo ocorre uma bifurcação do tipo sela-nó. A prova deste teorema usa alguns resultados obtidos do capítulo 3. Os diagramas de bifurcação estão nas páginas 147 e 148. A tabela de resultados está na página 149 .

O Teorema 34 da página 155 explica a bifurcação que ocorre no caso nó no bordo. A prova de que ocorre uma bifurcação sela-nó nesse caso depende de resultados do capítulo 3. Apresentamos o diagrama de bifurcação na página 156, não colocamos uma tabela por se tratar de um caso isolado.

O Teorema 38 da página 173 explica a bifurcação que ocorre quando temos uma tangência dupla externa que tenha número de Coll-Gasull-Prohens negativo. A regularização possui uma bifurcação de Hopf com primeiro número de Lyapunov negativo. O diagrama de bifurcação da regularização está na página 175 .

Ao longo desta tese explicamos as bifurcações de alguns campos vetoriais descontínuos via o método da regularização. O estudo das bifurcações dos outros casos apresentados em $[\mathrm{KGR}]$ podem ser explicados do mesmo modo como os tratados nesta tese. 


\section{Glossário}

Caso Foco no bordo Um CVD a um parâmetro $Z_{\lambda}=\left(X_{\lambda}, Y_{\lambda}\right)$, onde $X_{\lambda}$ possui um foco repulsor em $N$, para valores pequenos e negativos de $\lambda$, que colide com o conjunto de descontinuidade. O campo $Y_{\lambda}$ é constante. Esse caso está dividido em seis subcasos denotados por $\mathcal{F}_{\rangle}, i=1,2, \ldots, 6$.

Caso Sela no bordo Um CVD a um parâmetro $Z_{\lambda}=\left(X_{\lambda}, Y_{\lambda}\right)$, onde $X_{\lambda}$ possui uma sela em $N$, para valores pequenos e negativos de $\lambda$, que colide com o conjunto de descontinuidade. O campo $Y_{\lambda}$ é constante. Denotamos esse caso por $\mathcal{S}$.

Nó no bordo Um CVD a um parâmetro $Z_{\lambda}=\left(X_{\lambda}, Y_{\lambda}\right)$, onde $X_{\lambda}$ possui um nó atrator em $N$, para valores pequenos e negativos de $\lambda$, que colide com o conjunto de descontinuidade. O campo $Y_{\lambda}$ é constante. Denotamos esse caso por $\mathcal{N}$.

Caso Sela e Nó no bordo Um CVD a um parâmetro $Z_{\lambda}=\left(X_{\lambda}, Y_{\lambda}\right)$, onde o campo de Filippov, $F_{Z_{\lambda}}$, possui uma sela e um nó atrator em $D$, para valores pequenos e negativos de $\lambda$, que colidem quando o parâmetro se anula. O campo $Y_{\lambda}$ é constante. Denotamos esse caso por $\mathcal{S N}$.

Caso Duas tangências Externas Um CVD a um parâmetro $Z_{\lambda}=\left(X_{\lambda}, Y_{\lambda}\right)$, onde os campos $X_{\lambda}$ e $Y_{\lambda}$ possuem uma tangência quadrática externa com o conjunto de descontinuidade. Denotamos esse caso por $\mathcal{T}$. 


\section{Referências Bibliográficas}

[A] A. A. Andronov, E. Leontovich, I. Gordon e A. Maier, Theory of Bifurcation of Dynamical Systems on a Plane, I.P.S.T, Jerusalém, (1971).

[A2] A. A. Andronov, E. Leontovich, I. Gordon e A. Maier, Qualitative Theory of SecondOrder Dynamic Systems, I.P.S.T, Jerusalém, (1973).

[AVK] A. A. Andronov, A. A. Vitt, S. E. Khaikin, Theory of Oscillators, Pergamon Press, (1966).

[BBCK] M. di Bernardo, C. J. Budd, A. R. Champneys, P. Kowalczyk, Piecewise Smooth Dynamical Systems Theory and Applications, Springer-Verlag, (2008).

[CGP] B. Coll, A. Gasull e R. Prohens, Degenerate Hopf Bifurcations in Discontinuous Planar Systems, Journal of Mathematical Analysis and Applications, 253, 671-690, (2001).

[F] A. F. Filippov, Differential Equations with Discontinuous Righthand Sides, Kluwer Academic Press, (1988).

[G] N. A. Gubar, Bifurcations in the Vicinity of a "Fused Focus", Journal of Appl. Math. Mech., 35, 890-895, (1971).

[GH] J. Guckenheimer e P. Holmes, Nonlinear Oscillations, Dynamical Systems, and Bifurcation of Vector Fields, Springer-Verlag, Nova Iorque, (1983).

[K] Yu. A. Kuznetsov, Elements of Applied Bifurcation Theory, Springer, 3rd edition, (2004).

[KGR] Yu. A. Kuznetsov, A. Gragnani e S. Rinaldi, One-Parameter Bifurcations in Planar Filippov Systems, Int. Journal of Bifurcation and Chaos, vol. 13, No. 8: 2157-2188, (2003). 
[M] A. L. F. Machado, Estabilidade Estrutural e Bifurcações de Campos Vetoriais Descontínuos, Tese IME-USP, (2000).

[P] L. Perko, Differential Equations and Dynamical Systems, Springer, 3rd edition, (2001).

[S1] J. Sotomayor, Curvas Definidas por Equações Diferenciais no Plano, 13o Colóquio Brasileiro de Matemática, IMPA, Rio de Janeiro, (1981).

[S2] J. Sotomayor, Lições de Equações Diferenciais Ordinárias, IMPA, Projeto Euclides, (1979).

[S3] J. Sotomayor, Generic One-Parameter Families of Vector Fields on Two Dimensional Manifolds, Publications Mathématiques de L'Institut des Hautes Études Scientifiques, França, v. 43, p. 05-46, (1974).

[S4] J. Sotomayor, Generic bifurcations of dynamical systems, In Dynamical Systems, M. M. Peixoto (ed.), Academic Press, Nova Iorque, 561-582, (1971).

[SM] J. Sotomayor, A. L. F. Machado, Structurally Stable Discontinuous Vector Fields in the Plane, Qualitative Theory of Dynamical Systems, Lleida, v. 3, p. 227-250, (2002).

[ST] J. Sotomayor, M. A. Teixeira, Regularization of Discontinuous Vector Fields, World Scientific Publishing River Edge, Estados Unidos, p. 207-223, (1998). 